\title{
AN INVESTIGATION OF INITIALLY DELAMINATED COMPOSITE SANDWICH WITH DELAMINATION ARREST MECHANISM UNDER BUCKLING LOADING
}

\author{
A Thesis \\ Presented to the Faculty of \\ Aerospace Engineering Department \\ California Polytechnic State University, San Luis Obispo
}

In Partial Fulfillment of the Requirements

for the Degree of Master of Science

in Aerospace Engineering

By

Tony D. Tran

December 2010 
(C) 2010

Tony D. Tran

ALL RIGHTS RESERVED 


\section{COMMITTEE MEMBERSHIP}

Title:

Author:

Date Submitted:

Committee Chair:

Committee Member:

Committee Member:

Committee Member:

Committee Member:
An Investigation of Initially Delaminated Composite Sandwich with Delamination Arrest Mechanism under Buckling Loading

Tony D. Tran

September 2010

Faysal Kolkailah, Ph.D., P.E.

Eltahry Elghandour, Ph.D.

Eric Mehiel, Ph.D.

Susan Opava, Ph.D.

Eric Kasper, Ph.D., P.E. 


\begin{abstract}
An Investigation of Initially Delaminated Composite Sandwich with Delamination Arrest Mechanism under Buckling Loading

By

Tony D. Tran
\end{abstract}

This thesis involves the development of a fiberglass-foam composite sandwich structure with the introduction of delamination arrestment keys; therefore, a study of an initially delaminated composite sandwich structure was the experimental analysis on multiple configurations in how the arrestment keys are placed.

The first part of this thesis research was to the experimental design and manufacturing of the composite sandwich plates. These plates were later cut down to the specific test dimensions and manufacturing processes for the composite sandwich plates and test specimens were created. The composite sandwich plates were manufactured using a vacuum resin infusion process. The dimensions of the composite layup are 14 inches in length with a width of 10.75 inches. The width size has margin to account for machining. The actual dimensions of the test specimen after it is prepared are 14 inches by 0.75 inches. The test anvil length is 11 inches and is used to perform tests to determine mechanical characteristics of the structures under buckling loading. These plates provide approximately 9 to 13 specimens per each case. All the test specimens have 4 plies of 18 oz fiberglass woven roving fabric from Jamestown Distributors, a LAST-A-FOAM FR-6710 foam core, and 5 to 1 ratio of West Systems 105/206 epoxy. Also, a non-porous material was integrated into the structure to create an initial delamination in some of the case studies. The integration of the delamination arrestment keys involve milling the foam core to provide the necessary grooves for key placements before the structure is vacuumed and epoxy is flowed. The arrestment keys are made of unidirectional fiberglass strand and the West Systems 105/206 epoxy using a wet layup process. In addition, fiberglass woven roving specimens were created to see the material characteristics under compression and tensile loading. The same is created to determine the material properties of the foam core, wood boundary core, and arrestment keys under compression loading.

The second part to this thesis investigation is the experimental testing of the test specimens with all different variables considered. Those variables includes determining the final solid cure duration of the fiberglass skin, the geometric lengths between pure compression and pure buckling, behaviors of different initial delamination size, effects of continuous and discontinuous arrestment keys parallel and perpendicular to the in-plane loading, and material properties. The final solid cure duration differ from what the manufacturer gave on their epoxy. This experiment testing followed ASTM D-3039 standard to see the differences in elastic modulus over duration of 15 days. The resulting data shows that the test specimen fully cures after 13 to 14 days. The test specimens in search of the geometric buckling length for this investigation did not follow ASTM C-364 standard in full, but follows a variation of the ASTM C-364 standard in order to support buckling loading condition and the limited accessibility of the test equipments. Instead, the modifications are found with a different test jig design and test specimen configuration. The test jig was created to provide a pinned condition with a 0.25 inch diameter. The test specimen is laid up with a foam and wood cores. Two wood cores are laid at each edge of the foam core to increase loading capacity and holes are drilled through the wood cores to create a pinned-pinned case for the optimum buckling condition. The results detailing the geometric buckling show that after 9 inches anvil length there is no compression; only buckling occurs with a cross-sectional dimension of 0.75 inch by 0.575 inch. The 11 inch foam length was 
chosen for convenience of machining. This modified setup was also used for testing the different configuration with the embedded arrestment keys. The multiple different configurations completed for these test specimens under unstable loading, the experiment results show that a continuous arrestment key embedded significantly improve the loading capacity over a perfectly sound non-delaminated specimen and maintain the majority of loading capacity even with an introduced delamination. The embedded continuous key also provided a higher horizontal displacement capability before fracture in comparison to the initially delaminated test specimens. As for the test specimens used to determine the material characteristics, ASTM D-3410 and modified ASTM C-364 standards were followed. The test specimens had a fiber volume fraction of approximately 0.60 , which details the brittle failure under tensile and compression loading. The results also show that the fibrous fiberglass test specimens have a higher ultimate strength in compression or buckling then in tension.

All of the experimental testing was completed in the Aerospace Engineering Structural/Composite Lab at California Polytechnic State University at San Luis Obispo, California. Therefore, an introduction of a continuous arrestment key parallel to the in-plane loading and embedded into the composite sandwich structure provided a significant increase in loading and buckling capabilities in comparison to the control test specimens with and without an initial delamination and no embedded key. The continuous key placed parallel to the load vector increased the structural strength with an increase of $126 \%$ from a 1-inch delaminated structures and only an $11 \%$ drop from non-delaminated structures. That is, 1-inch and 2-inch delaminated structures showed a $61 \%$ drop and $81 \%$ drop from non-delaminated structures. Some configurations have reduced or arrested of the delaminated region. 


\section{ACKNOWLEDGEMENTS}

I would like to start off by thanking my thesis advisor, Dr. Faysal Kolkailah, and my professor, Dr. Eltahry Elghandour for providing and supporting me through this exciting research. I truly appreciate all the support with the suggestions and advice throughout this thesis project. Words cannot explain how much appreciation I have for the both of you. I would like to thank Dr. Eric Mehiel, Dr. Dianne DeTurris, and Dr. Susan Opava for supporting me and being a part of my thesis defense committee. I would also like to thank Dr. Eric Kasper for his advice and all the questions asked through this project. I am truly grateful for the overwhelming support, the wide knowledge base, and the major dedication through all the ins and outs of this thesis project.

Thank you to all my friends who believed in me and supported me since the beginning of my college career. It is quite indescribable how much impact it is to have that kind of support and motivation to succeed and go beyond. Special thank you goes out to Alvaro Nunez and Henry Ureh, you both know what I am talking about!

I would also like to give a huge thank you to my family. Without their support, love, and sense of direction, I would not be who I am today and where I am now. Thank you to my father and mother who taught me to never stop and achieve every goal I have. They were there through all the ups and downs of my life. Also, I would like to thank my sisters and brother who also believed in their brother (even though, sometimes they don't show it!).

Also, I would like to thank Ahmad Amini for being a great friend and his wise words of wisdom. Thank you to Mike Jacobson, Thomas Woo, and Jenny Shatts for their never ending support, the motivation, and being very patient through this long adventure. I would also like to thank Richard Balatbat and Dominic Surano for all their help and working together through all the ups and downs. Cody Thompson for his support in maintaining all the machines and being very generous with his time throughout this project; Matt Ales for milling the foam; and Vince 
Perry for lightening up the lab and his support. I truly appreciate the continuous support from Lucas Swift and Jamal Stewart through the hard times.

To conclude, I would also like to thank Bill Charlton and Brian Terrell for holding my position at the Boeing Company at Mesa, Arizona. I truly appreciate all your understanding and support through this past year. 


\section{Table of Contents}

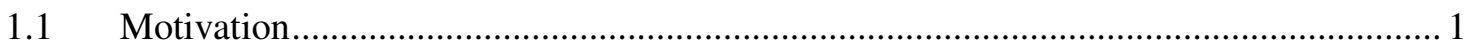

1.1.1 Problems and Reparation of Delamination for Composite Sandwich Structure ............ 1

1.1.2 Benefits of Delamination Arrest Keys ...................................................................... 5

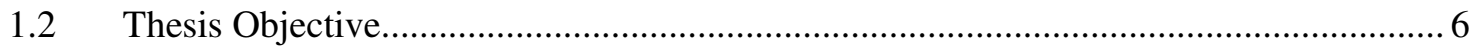

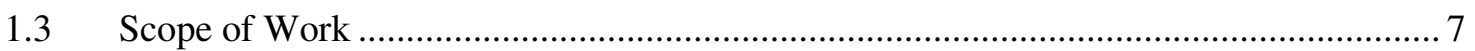

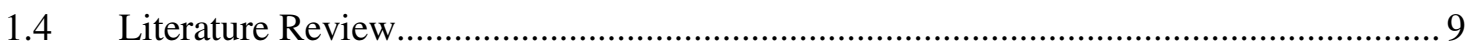

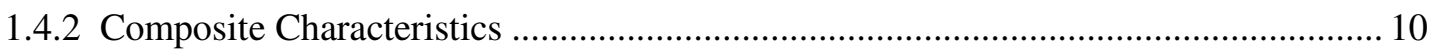

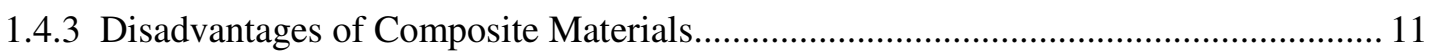

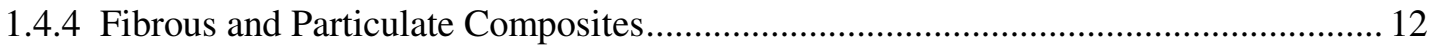

1.4.5 Application of Composite Sandwich Structures .......................................................... 15

1.4.6 Problems with Composite Sandwich Structures .......................................................... 16

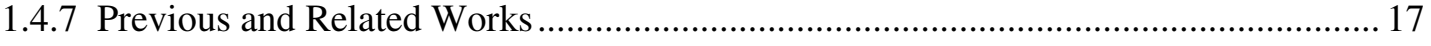

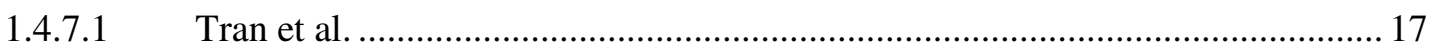

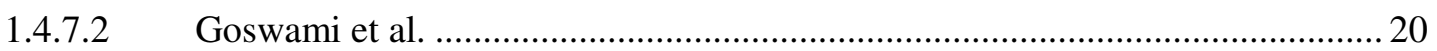

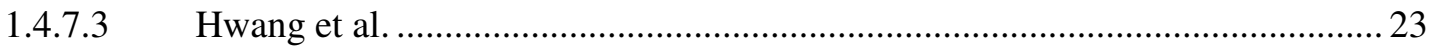

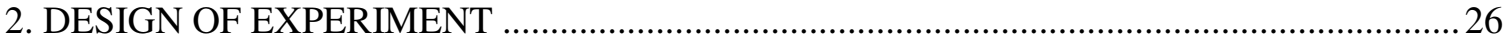

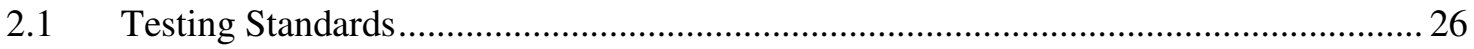

2.1.1 ASTM D-3039 Standard Test Method for Tensile Material Properties........................ 26

2.1.2 ASTM D-3410 Standard Test Method for Compressive Properties of Composite

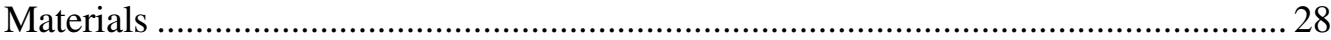

2.1.3 ASTM D-3171 Standard Test Methods for Constituent Content of Composite

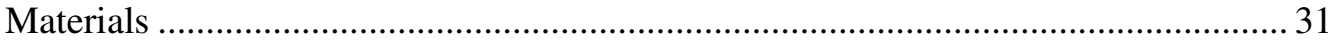

2.1.4 ASTM C-364 Standard Test Method for Edgewise Compressive Strength of

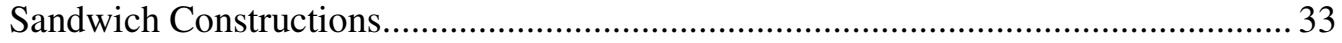

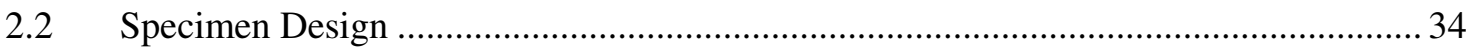

2.2.1 Component Designs of Composite Sandwich Structure …........................................... 35

2.2.1.1 Wood Core Component Design for Compressive Properties ............................ 35

2.2.1.2 E-Glass Composite Skin Component Design for Material Properties ................ 36

2.2.1.3 E-Glass Composite Design for Weight and Volume Fractions............................ 39

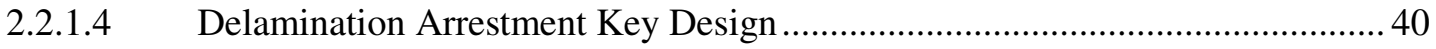

2.2.1.4.1 Delamination Arrestment Key Design for Mechanical Properties........................... 41

2.2.1.4.2 Delamination Arrestment Key Mold Design for Manufacturing ............................. 42 


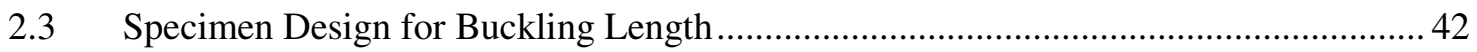

$2.4 \quad$ Specimen Design for Different Configurations ............................................................. 44

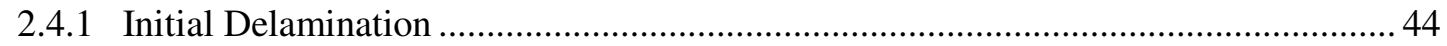

2.4.2 Delamination Arrestment Key Embedment and Foam Core Grooves .......................... 45

2.5 Material Selection and Mechanical Characteristics/Properties ..................................... 48

2.5.1 Foam Core Material Selection and Properties ............................................................ 49

2.5.2 Wood Core Material Selection and Properties........................................................... 50

2.5.3 Fiberglass Composite Material Selection and Properties............................................. 51

2.5.4 Epoxy Material Selection and Characteristics ........................................................... 52

2.5.5 E-Glass Composite Skin Properties and Characteristics.............................................. 52

2.5.5.1 Weight and Volume Fractions of E-Glass Skin .............................................. 53

2.5.6 Delamination Arrestment Key Properties and Characteristics........................................ 54

2.5.6.1 Weight and Volume Fractions of Delamination Arrestment Key........................54

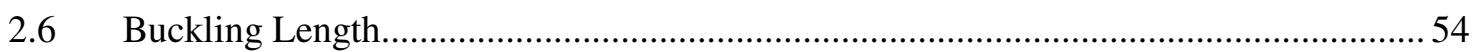

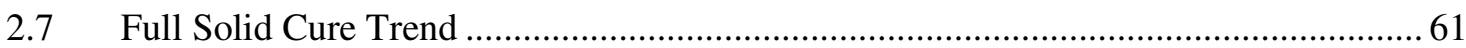

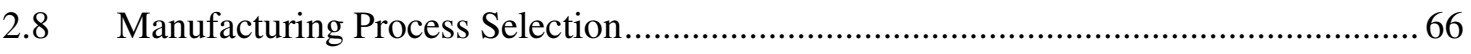

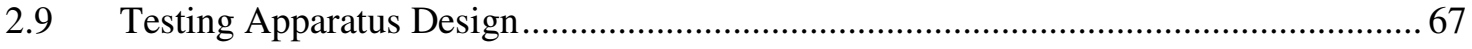

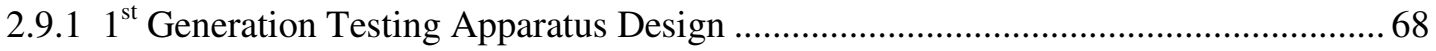

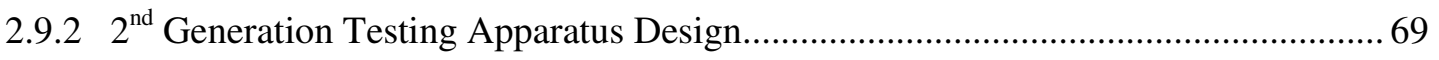

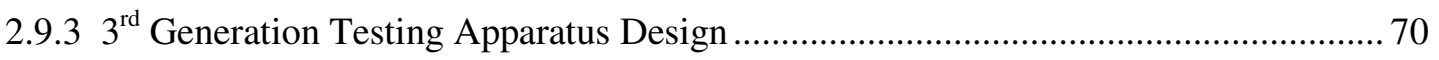

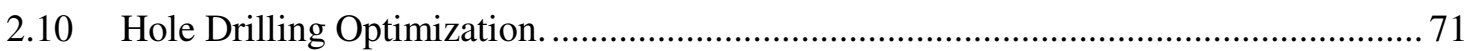

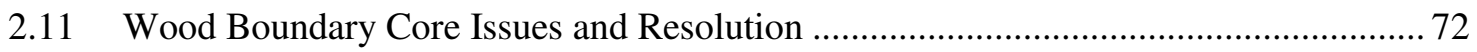

3. MANUFACTURING, PREPPING, AND TESTING PROCEDURES ……............................ 74

3.1 Manufacturing and Prepping Procedures ................................................................. 74

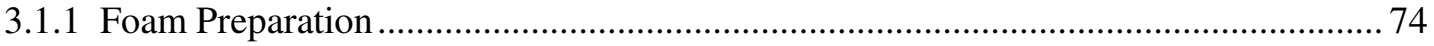

Approximately 40 foam plates were cut down to size and use to manufacture all the test

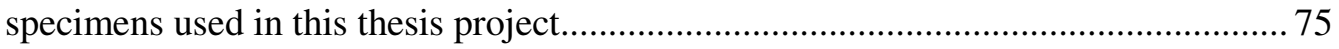

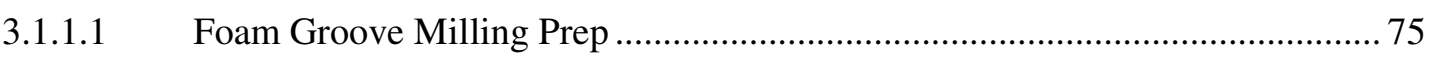

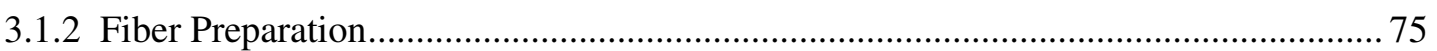

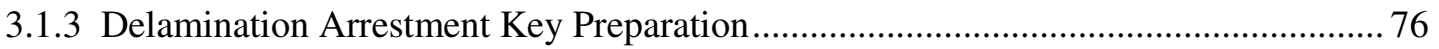

3.1.4 Composite Strip Preparation for Mechanical Properties Testing................................ 81

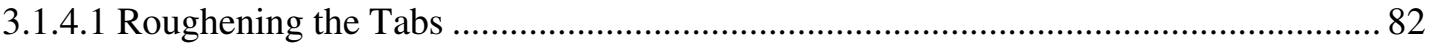

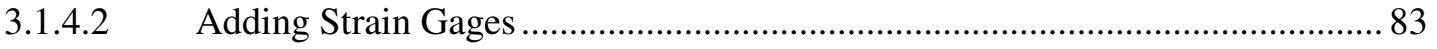

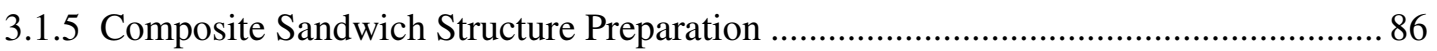

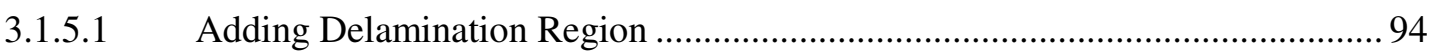

3.1.5.2 Embedding Delamination Arrestment Key ................................................... 95 


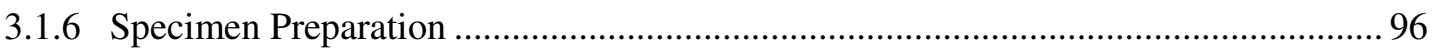

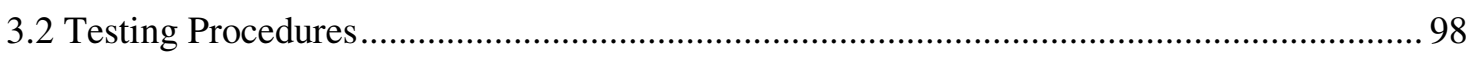

3.2.1 Weight and Volumetric Fraction Procedure Testing for Face Sheet .......................... 98

3.2.2 Weight and Volumetric Fraction Procedure Testing for Arrestment Keys................... 99

3.2.3 Composite Skin Testing for Material Properties.......................................................... 100

3.2.4 Testing for Material Properties using Plate-Plate Boundary ..................................... 100

3.2.5 Testing for Mechanical Behaviors and Properties using Pinned-Pinned Boundary .. 101

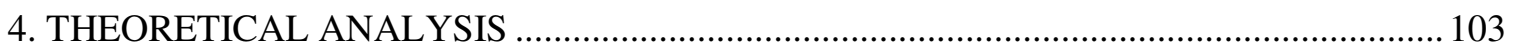

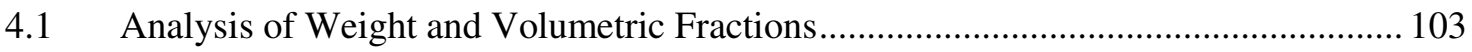

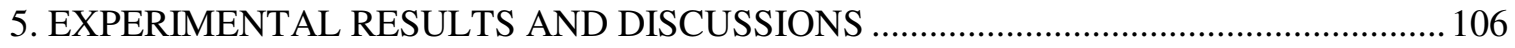

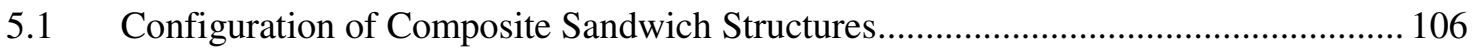

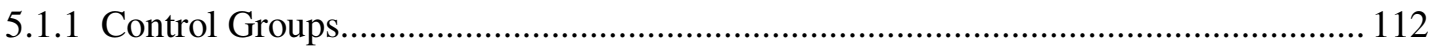

5.1.1.1 Control Group: No Delamination and No Embedded Arrestment Key ............ 112

5.1.1.2 Control Group: Initial Delamination with No Embedded Arrestment Key .............. 114

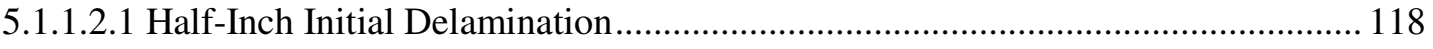

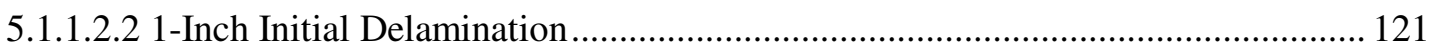

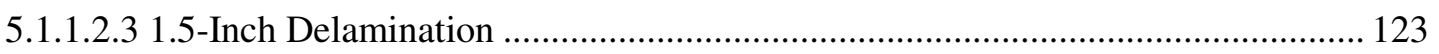

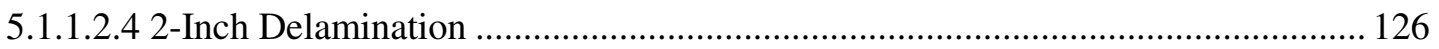

5.1.1.3 Control Group: No Delamination with Embedded Arrestment Key ................. 128

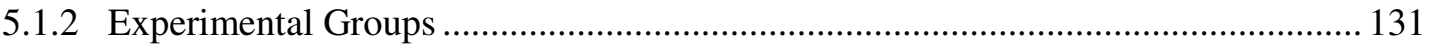

5.1.2.1 Experimental Group: Continuous Key in Vertical Direction with Skin

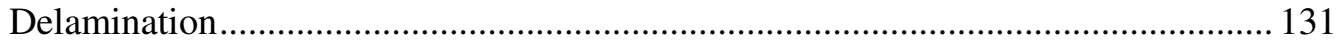

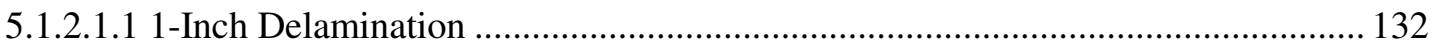

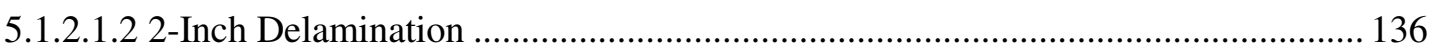

5.1.2.2 Experimental Group: Continuous Key in Vertical Direction with Key Delamination 139

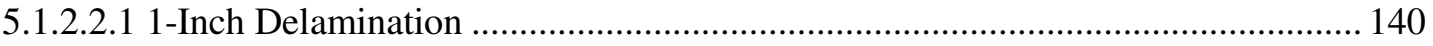

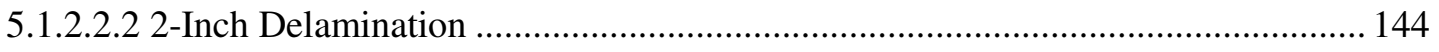

5.1.2.3 Experimental Group: Continuous Key in Horizontal Direction with Skin

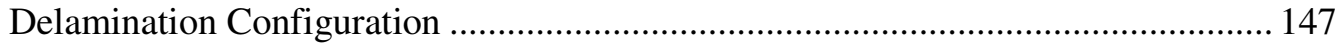

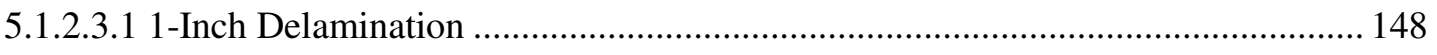

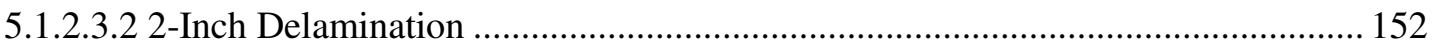

5.1.2.4. Experimental Group: Discontinuous Key in Vertical Direction with Skin

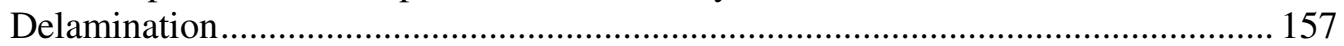

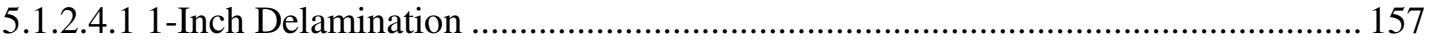

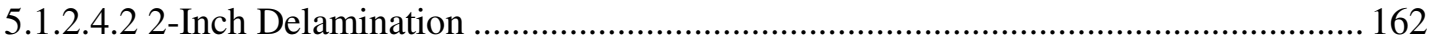

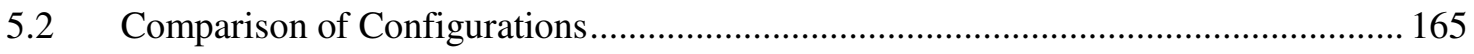


5.2.1 Comparison between '11 inches' and 'ID' Configurations ........................................ 167

5.2.2 Comparison between '11 inches' and 'ND' Configurations....................................... 167

5.2.3 Comparison between 'ND', 'SD', and 'KD' Configurations ..................................... 168

5.2.4 Comparison between 'ID', 'PL', and 'HD' Configurations ....................................... 169

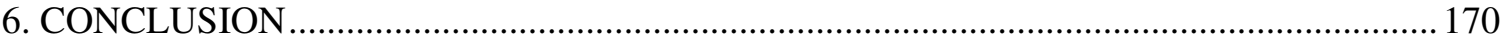

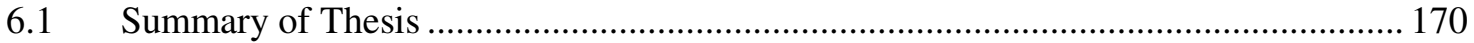

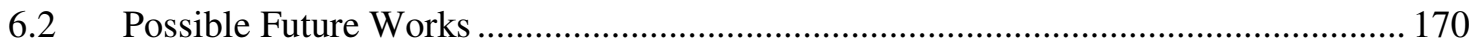

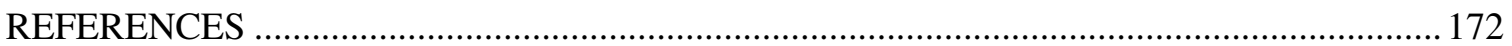

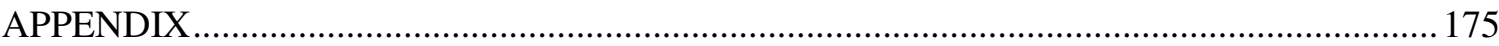

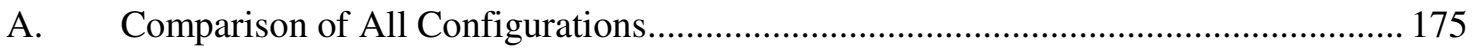

B. Thermolyne Type 1300 Muffle Furnace Specifications [31] .................................... 176 


\section{$\underline{\text { List of Tables }}$}

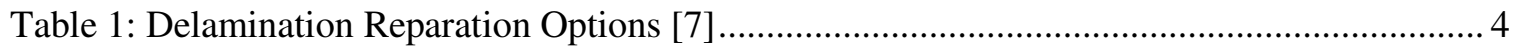

Table 2: Dimensions and Material Properties for Goswami et al. [26] ........................................ 22

Table 3: Mechanical Characteristics for Carbon/Epoxy Composites ..........................................24

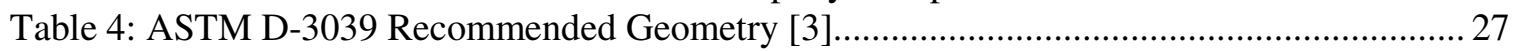

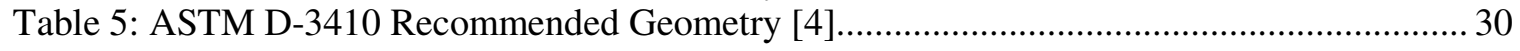

Table 6: Wood Core Design Dimensions for Compressive Properties........................................... 35

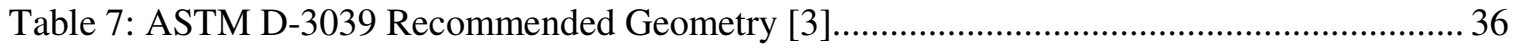

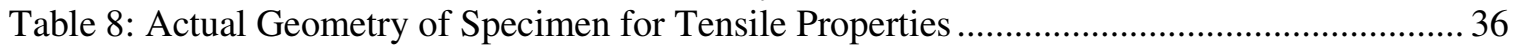

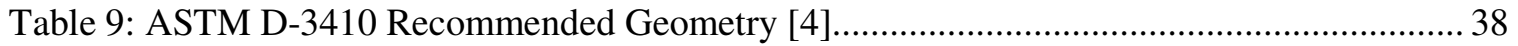

Table 10: Actual Geometry of Specimen for Tensile Properties ................................................... 38

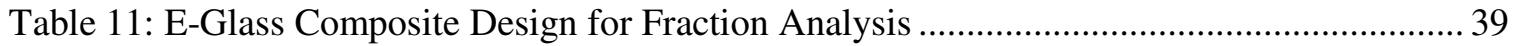

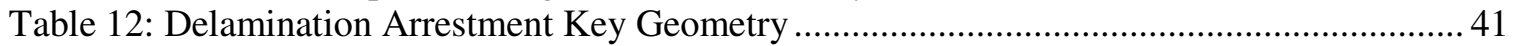

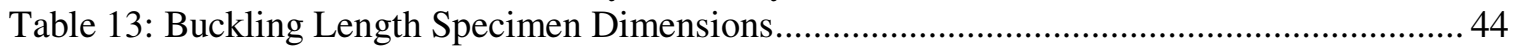

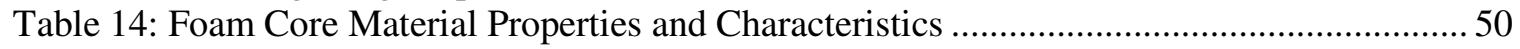

Table 15: Wood Core Material Properties and Characteristics................................................... 51

Table 16: Fiberglass Composite Material Properties and Characteristics [30] ............................. 51

Table 17: Epoxy Material Properties and Characteristics [29] ....................................................5 52

Table 18: Fiberglass Skin Mechanical Properties and Characteristics [29] ...................................53

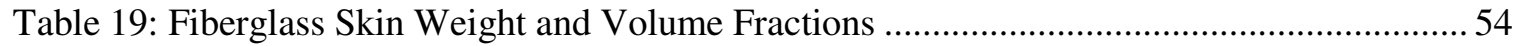

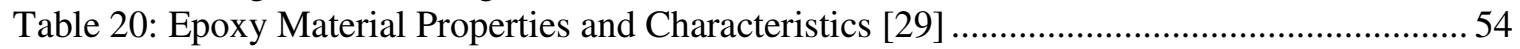

Table 21: Delamination Arrestment Key Weight and Volume Fractions ..................................... 54

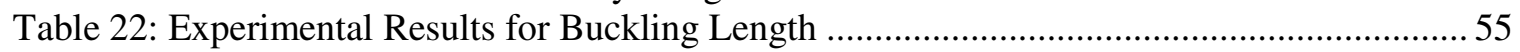

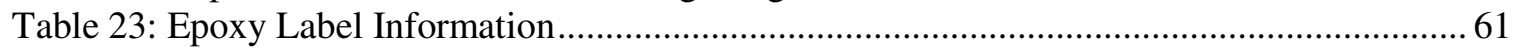

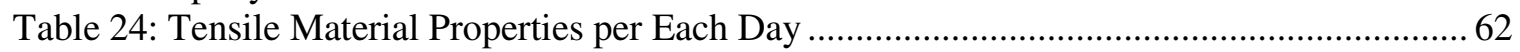

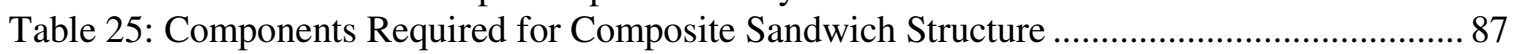

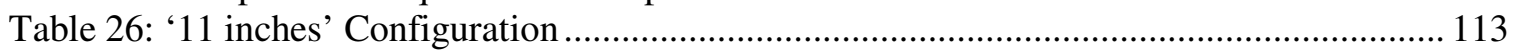

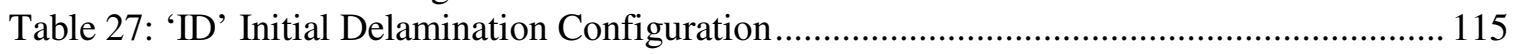

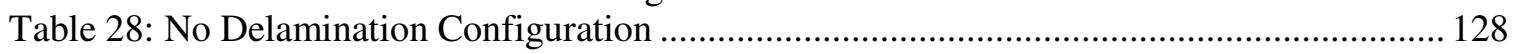

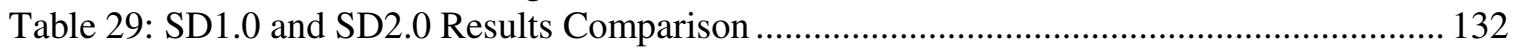

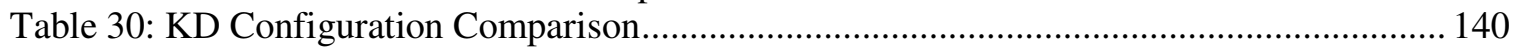

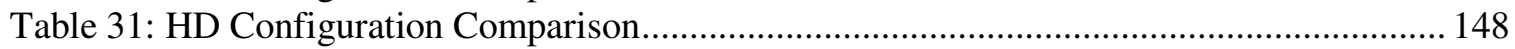

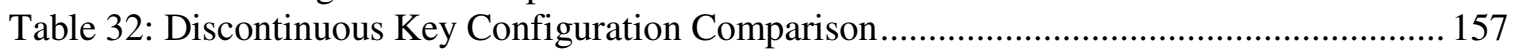

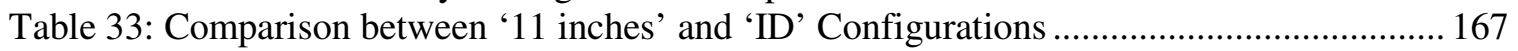

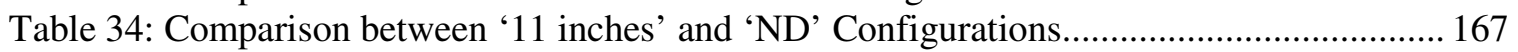

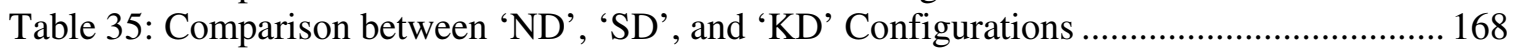

Table 36: Comparison between 'ID', 'PL', and 'HD' Configurations .......................................... 169 


\section{List of Figures}

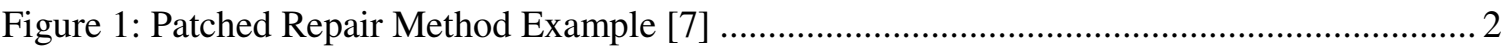

Figure 2: Taper Sanded or Scarf Repair Method Example [5] ................................................. 3

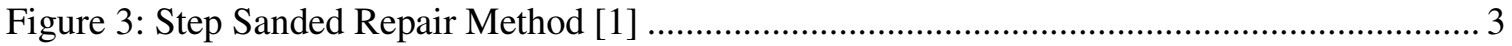

Figure 4: Advanced Composite Material Uses Example ............................................................... 10

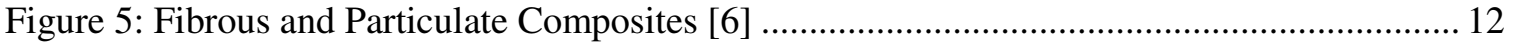

Figure 6: Examples of Continuous Fiberglass Materials [17-19] ............................................... 14

Figure 7: Examples of Application of Continuous Fiber Materials $[1,20]$................................... 14

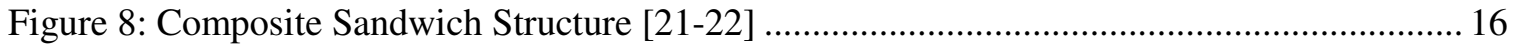

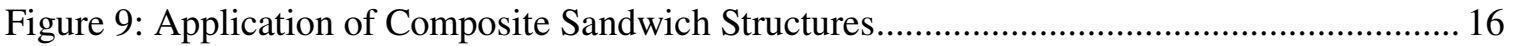

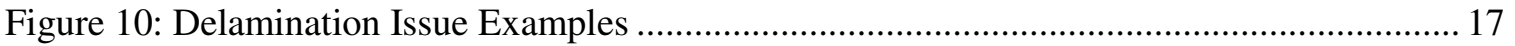

Figure 11: Examples of Materials used in Project by Tran et al. [25] .......................................... 18

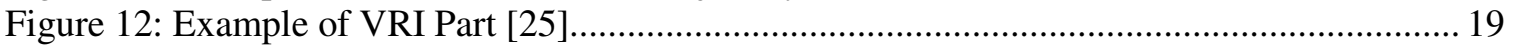

Figure 13: Mold and Fiberglass Strand Layup for Arrestment Keys [25] .................................... 19

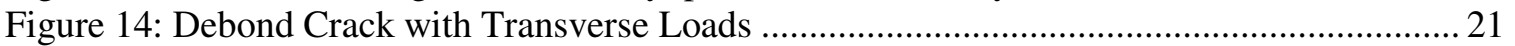

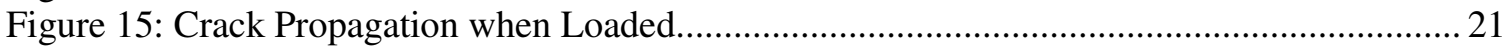

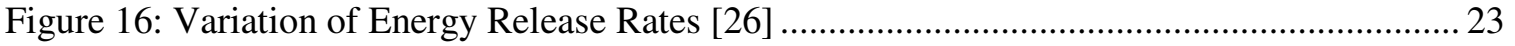

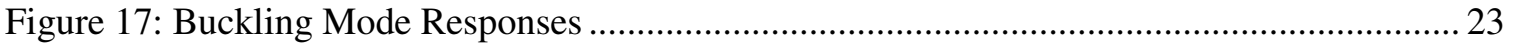

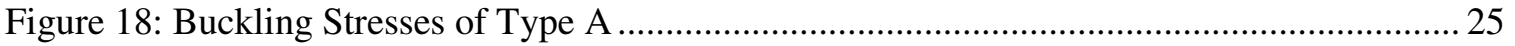

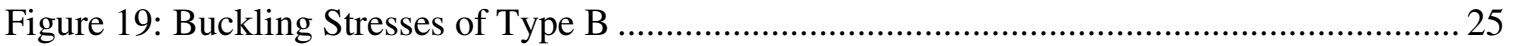

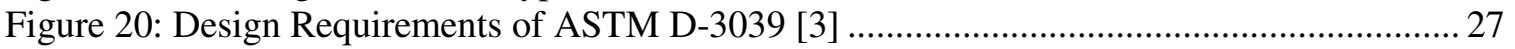

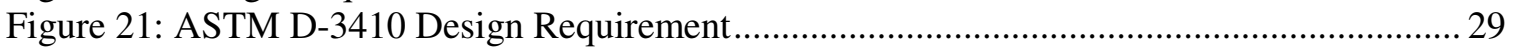

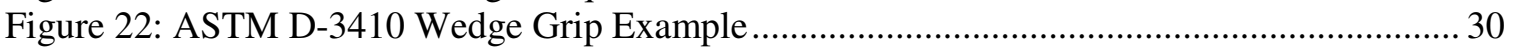

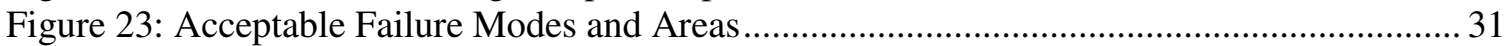

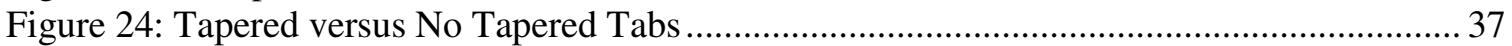

Figure 25: E-Glass Deviated Design for Compressive Properties ................................................ 39

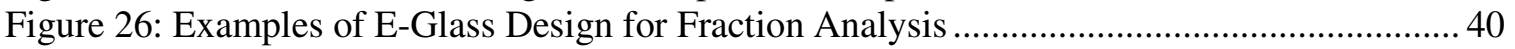

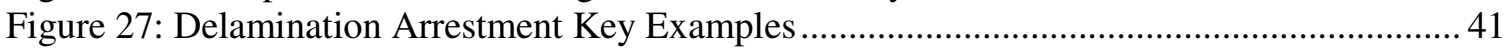

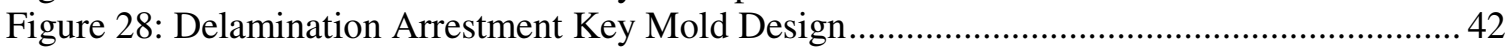

Figure 29: Second Generation Specimen and Wood Grain Issue ............................................... 43

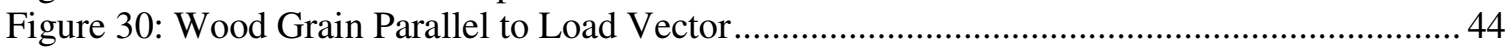

Figure 31: Horizontal Groove Configuration in Foam Core.................................................. 46

Figure 32: Discontinuous Vertical Groove Configuration in Foam Core .................................... 46

Figure 33: Full Continuous Groove Configuration in Foam Core .............................................. 47

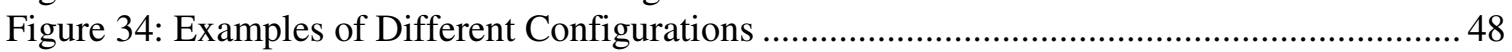

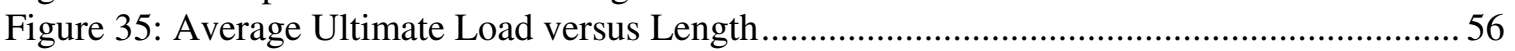

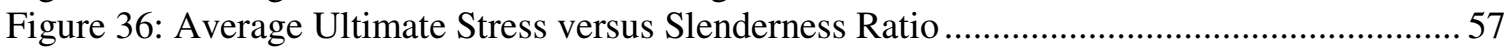

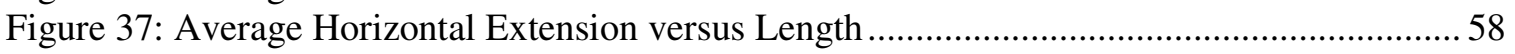

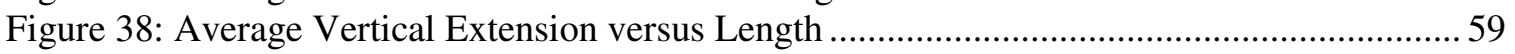

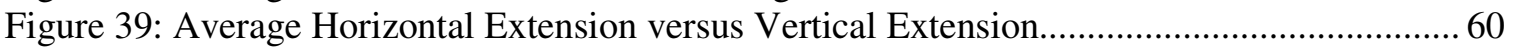

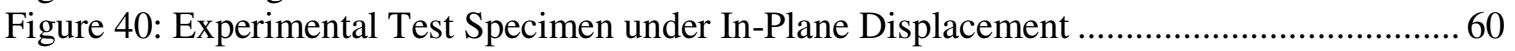

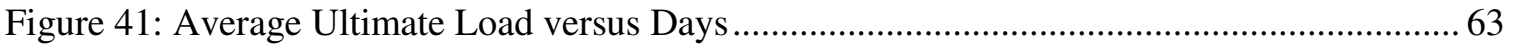

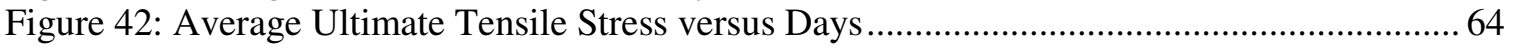

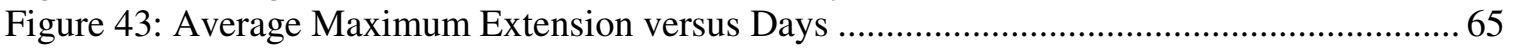

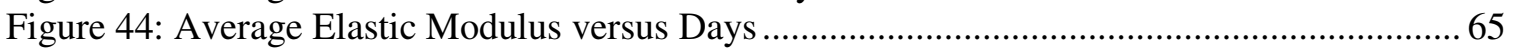

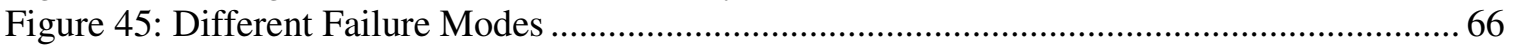

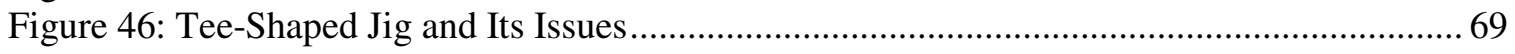

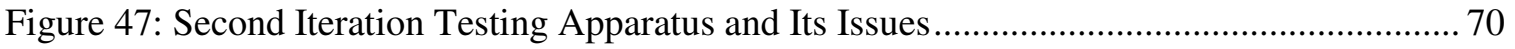




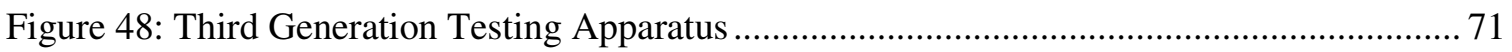

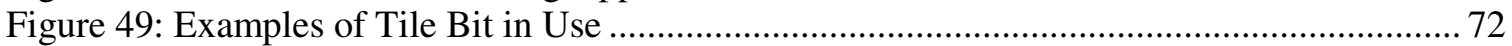

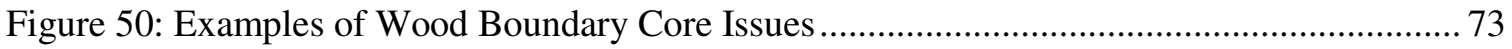

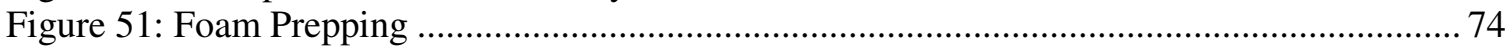

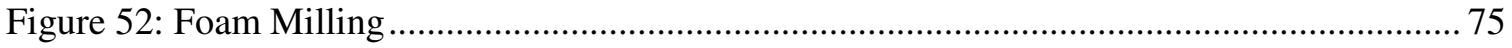

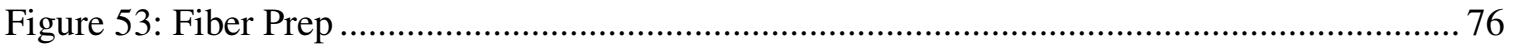

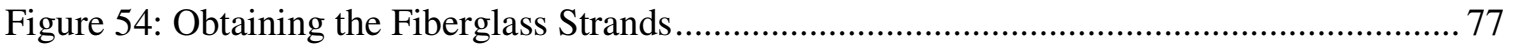

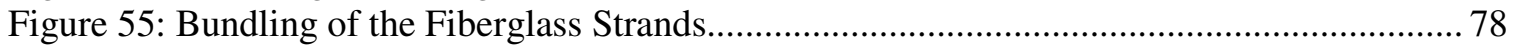

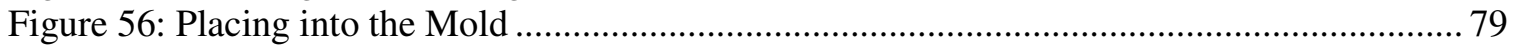

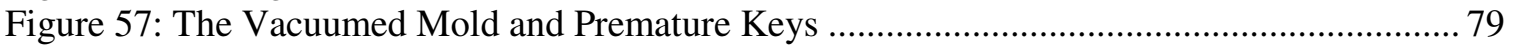

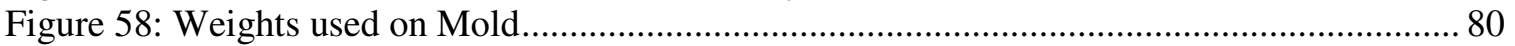

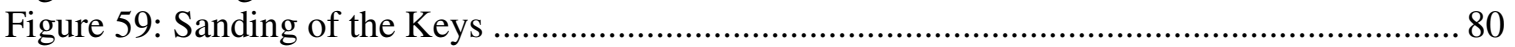

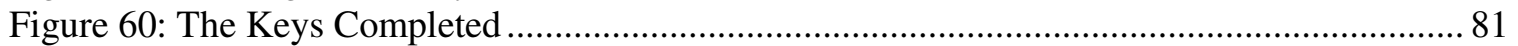

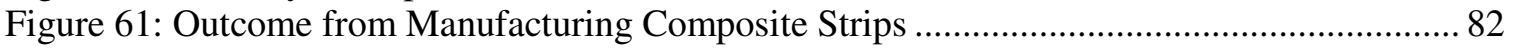

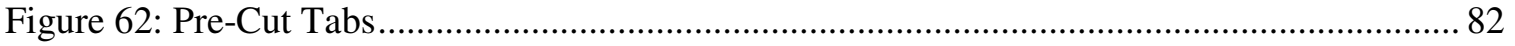

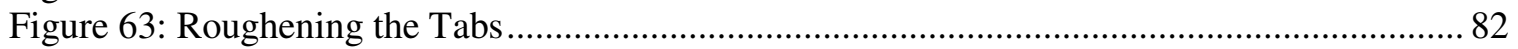

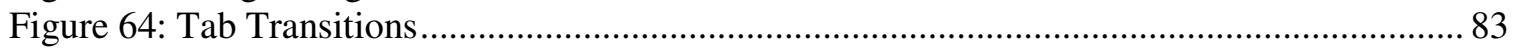

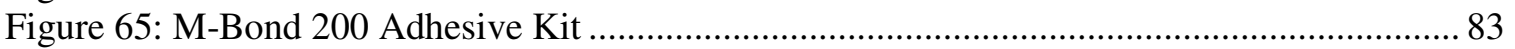

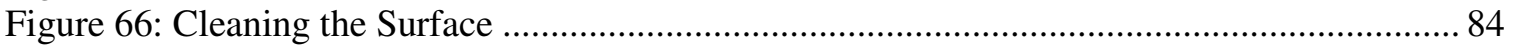

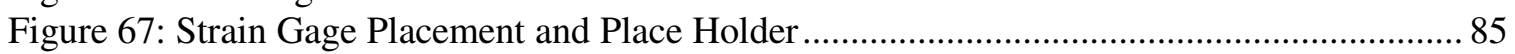

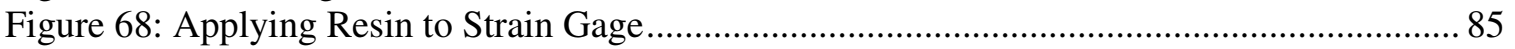

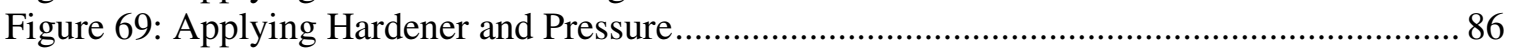

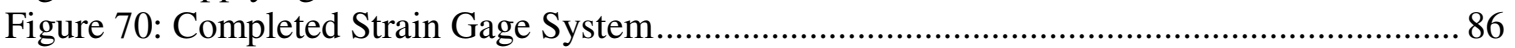

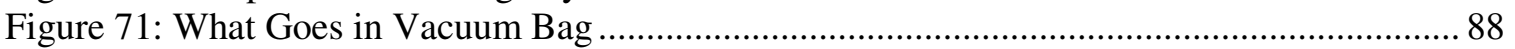

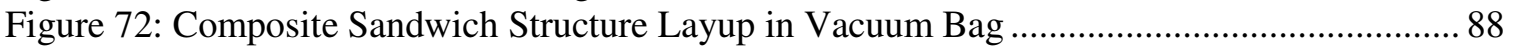

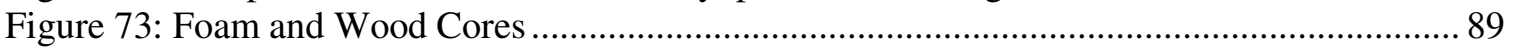

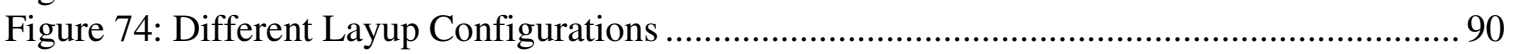

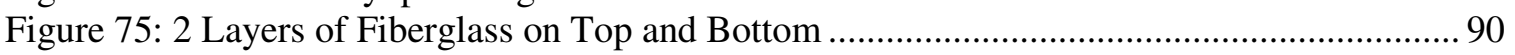

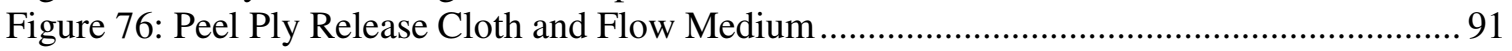

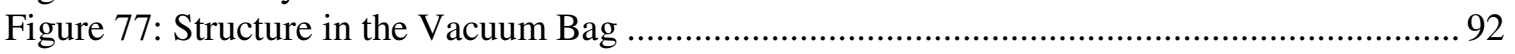

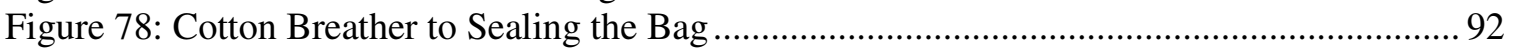

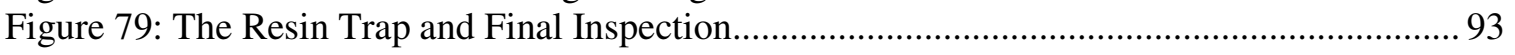

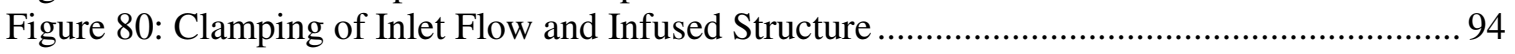

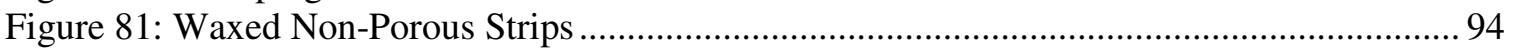

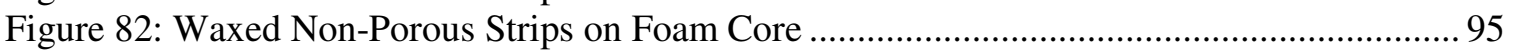

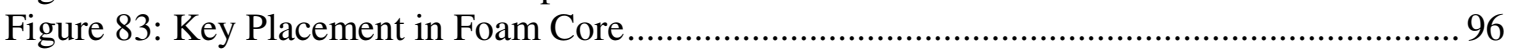

Figure 84: Epoxy Filler and Key Embedment for 'PL' Configuration ........................................ 96

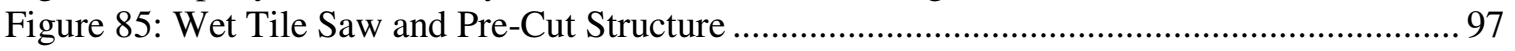

Figure 86: Cut Down Specimens and Drilled Holes............................................................... 98

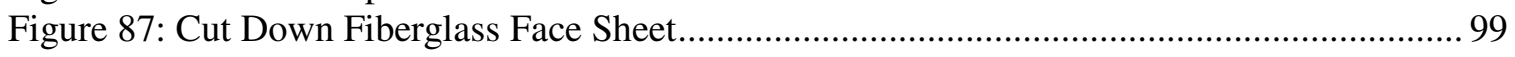

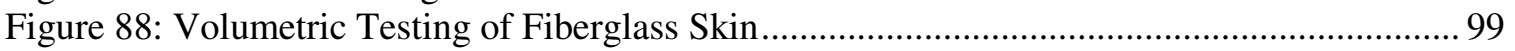

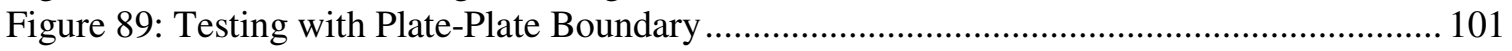

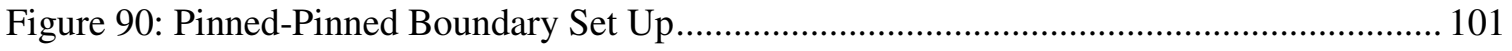

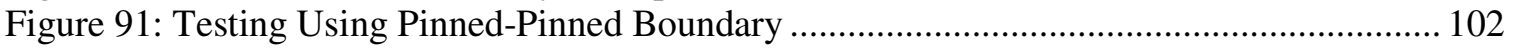

Figure 92: No Delamination and No Embedded Arrestment Key ............................................... 107

Figure 93: Initial Delamination with No Embedded Arrestment Key .......................................... 108

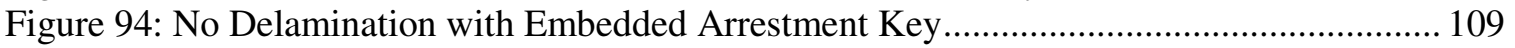

Figure 95: Continuous Key in Vertical Direction with Skin Delamination.................................. 110

Figure 96: Continuous Key in Vertical Direction with Key Delamination .................................. 110

Figure 97: Continuous Key in Horizontal Direction with Skin Delamination Configuration ..... 111

Figure 98: Discontinuous Key in Vertical Direction with Skin Delamination ............................ 112 


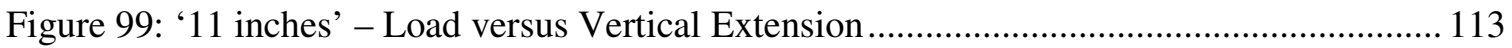

Figure 100: ‘11 inches' - Load versus Vertical Extension .......................................................... 114

Figure 101: '11 inches' - Load versus Vertical Extension ........................................................... 114

Figure 102: Mean Ultimate Load per Case ............................................................................. 116

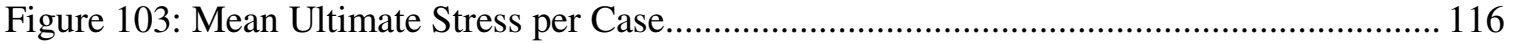

Figure 104: Mean of Maximum Vertical Extension per Case …............................................... 117

Figure 105: Mean of Maximum Vertical Extension per Case …................................................ 118

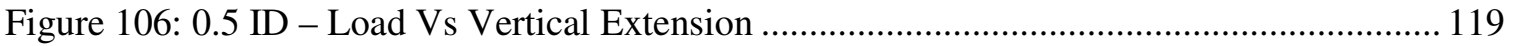

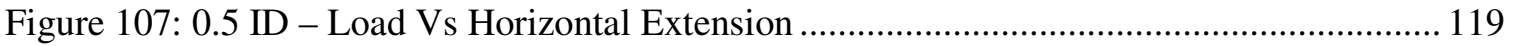

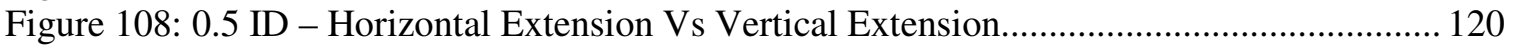

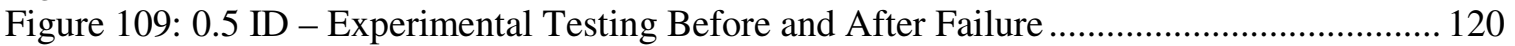

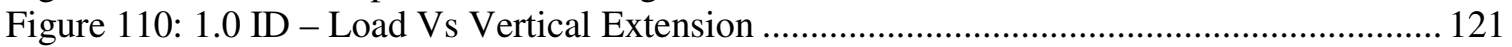

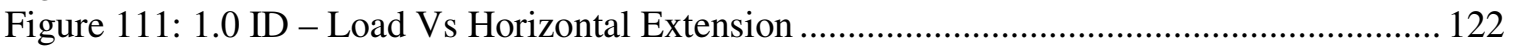

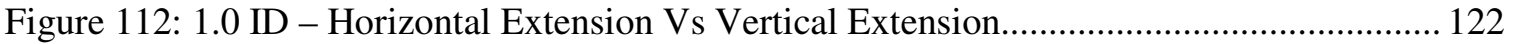

Figure 113: 1.0 ID - Experimental Testing Before and After Failure ......................................... 123

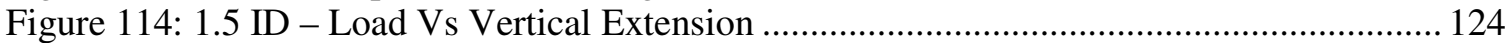

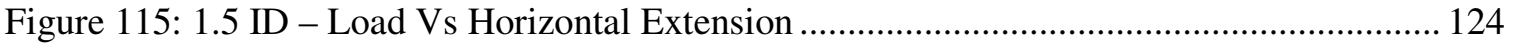

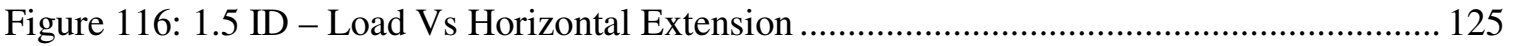

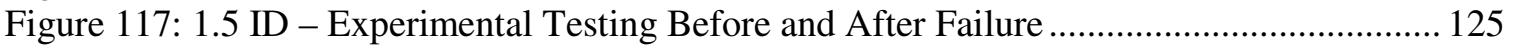

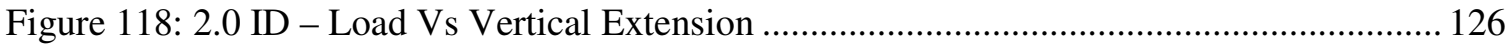

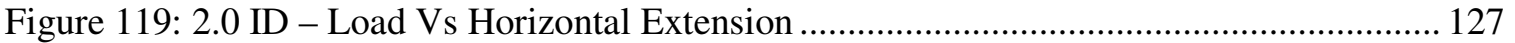

Figure 120: 2.0 ID - Horizontal Extension Vs Vertical Extension............................................. 127

Figure 121: 2.0 ID - Experimental Testing Before and After Failure ........................................ 128

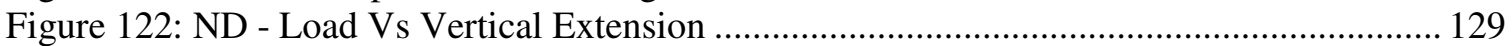

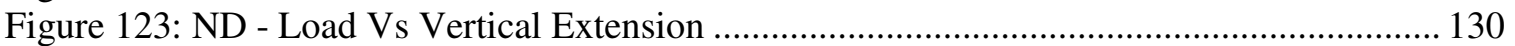

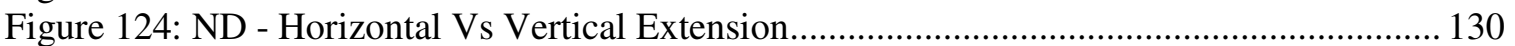

Figure 125: ND - Experimental Testing Before and After Failure .......................................... 131

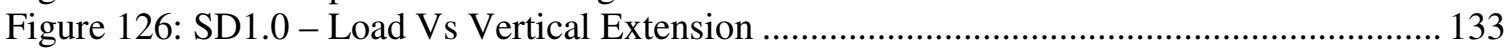

Figure 127: SD1.0 - Load Vs Horizontal Extension: On Delamination Side ............................. 134

Figure 128: SD1.0 - Load Vs Horizontal Extension: On Non-Delamination Side ..................... 134

Figure 129: SD1.0 - Horizontal Extension Vs Vertical Extension: On Delamination Side........ 135

Figure 130: SD1.0 - Horizontal Extension Vs Vertical Extension: On Non-Delamination Side 135

Figure 131: SD1.0 - Experimental Testing Before Failure ....................................................... 136

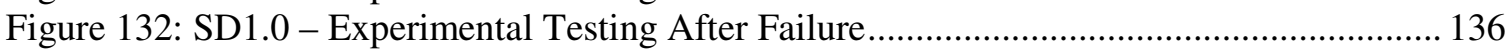

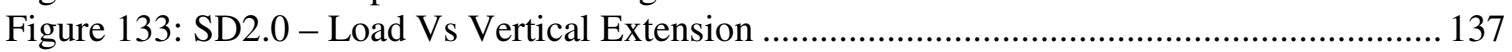

Figure 134: SD2.0 - Load Vs Horizontal Extension ................................................................ 138

Figure 135: SD2.0 - Horizontal Extension Vs Vertical Extension.............................................. 138

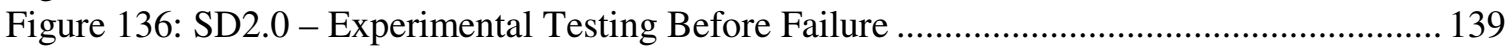

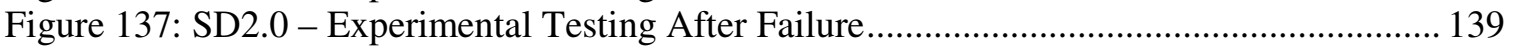

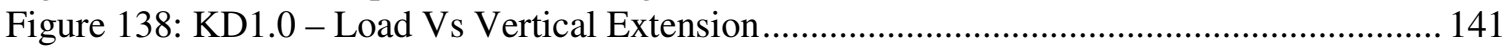

Figure 139: KD1.0 - Load Vs Horizontal Extension: On Delamination Side............................. 141

Figure 140: KD1.0 - Load Vs Horizontal Extension: On Non-Delamination Side....................... 142

Figure 141: KD1.0 - Horizontal Extension Vs Vertical Extension: On Delamination Side ........ 142

Figure 142: KD1.0 - Horizontal Extension Vs Vertical Extension: On Non-Delamination Side143

Figure 143: KD1.0 - Experiment Testing Before Failure ……................................................ 143

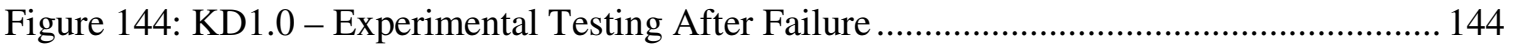

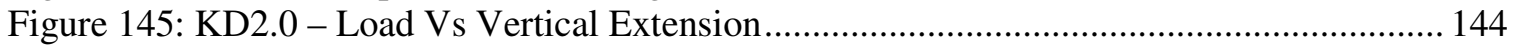

Figure 146: KD2.0 - Load Vs Horizontal Extension: On Delamination Side .............................. 145

Figure 147: KD2.0 - Load Vs Horizontal Extension: On Non-Delamination Side...................... 145

Figure 148: KD2.0 - Horizontal Extension Vs Vertical Extension: On Delamination Side ....... 146

Figure 149: KD2.0 - Horizontal Extension Vs Vertical Extension: On Non-Delamination Side146 


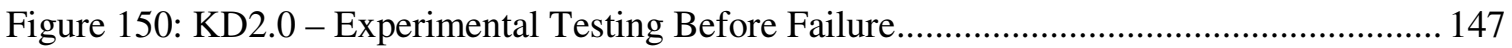

Figure 151: KD2.0 - Experimental Testing After Failure ........................................................ 147

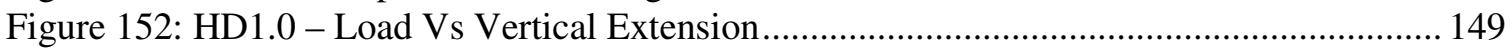

Figure 153: HD1.0 - Load Vs Horizontal Extension: On Delamination Side................................ 150

Figure 154: HD1.0 - Load Vs Horizontal Extension: On Non-Delamination Side...................... 150

Figure 155: HD1.0 - Load Vs Horizontal Extension: On Non-Delamination Side...................... 151

Figure 156: HD1.0 - Horizontal Extension Vs Vertical Extension: On Non-Delamination Side151

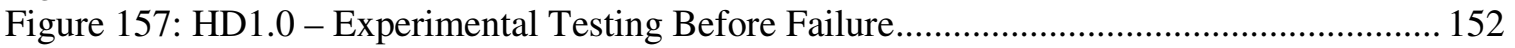

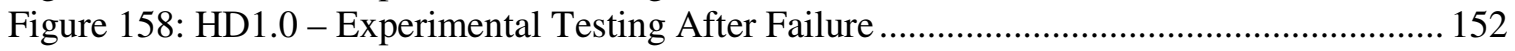

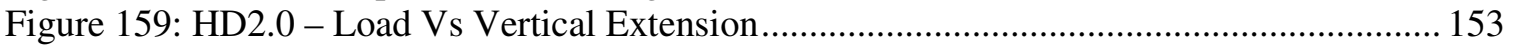

Figure 160: HD2.0 - Load Vs Horizontal Extension: On Delamination Side ............................... 153

Figure 161: HD2.0 - Load Vs Horizontal Extension: On Non-Delamination Side...................... 154

Figure 162: HD2.0 - Horizontal Extension Vs Vertical Extension: On Delamination Side ....... 155

Figure 163: HD2.0 - Horizontal Extension Vs Vertical Extension: On Non-Delamination Side155

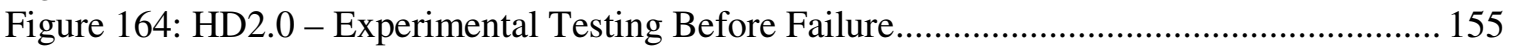

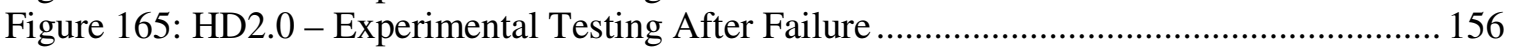

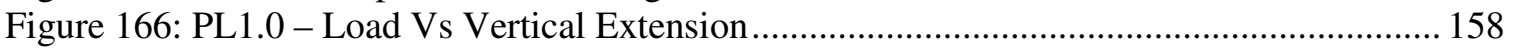

Figure 167: PL1.0 - Load Vs Horizontal Extension: On Delamination Side ............................... 159

Figure 168: PL1.0 - Load Vs Horizontal Extension: On Non-Delamination Side........................ 159

Figure 169: PL1.0 - Horizontal Extension Vs Vertical Extension: On Delamination Side ........ 160

Figure 170: PL1.0 - Horizontal Extension Vs Vertical Extension: On Non-Delamination Side 160

Figure 171: PL1.0 - Experimental Testing Before Failure ......................................................... 161

Figure 172: PL1.0 - Experimental Testing After Failure ............................................................ 161

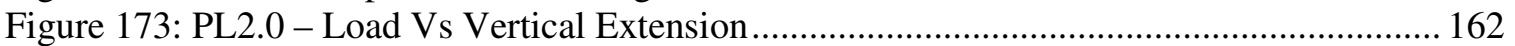

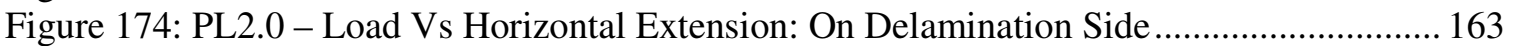

Figure 175: PL2.0 - Load Vs Horizontal Extension: On Non-Delamination Side...................... 163

Figure 176: PL2.0 - Horizontal Extension Vs Vertical Extension: On Delamination Side ........ 164

Figure 177: PL2.0 - Horizontal Extension Vs Vertical Extension: On Non-Delamination Side 164

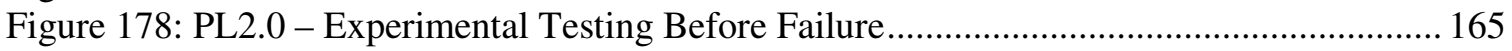

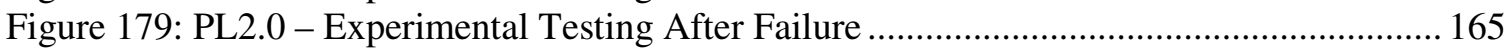

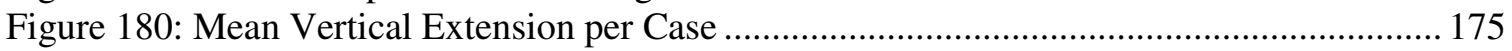

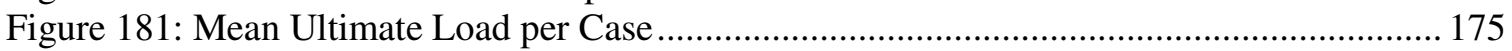

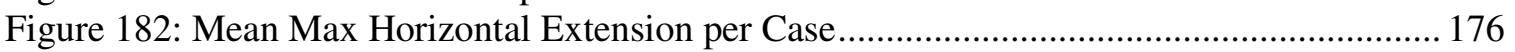

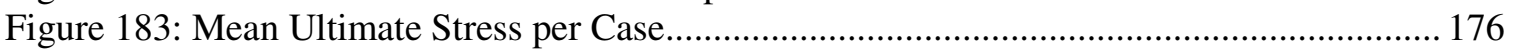




\section{List of Equations}

Equation 1: Weight Fraction and Weight of Composite Structure ............................................ 103

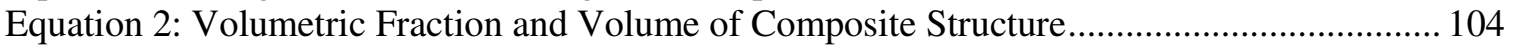

Equation 3: Relationship between Weight Fractions and Volumetric Fractions .......................... 104

Equation 4: Relationship between Component Weight Fractions and Volumetric Fractions...... 104

Equation 5: Elastic Modulus using Volumetric Fractions ........................................................ 105 


\section{List of Symbols}

$\begin{array}{lll}\text { E } & \text { Elastic Modulus } & (\mathrm{psi}) \\ \mathrm{W} & \text { Weight Fraction } & (-) \\ \mathrm{W} & \text { Weight } & (\mathrm{lbf}) \\ \mathrm{V} & \text { Volume Fraction } & (-) \\ \mathrm{v} & \text { Volume } & \left(\mathrm{in}^{3}\right) \\ \rho & \text { Density } & \left(\mathrm{lbs} / \mathrm{in}^{3}\right) \\ v & \text { Poisson's Ratio } & (-)\end{array}$

\section{Subscripts}

$\mathrm{f}$

Fiber

lat Lateral Direction

long Longitudinal Direction

m Matrix

tot Total 
(This page is intentionally left blank) 


\section{INTRODUCTION}

\subsection{Motivation}

Delamination within composite sandwich structure is quite common and usually caused by manufacturing defects, foreign object damages, and even prolonged environmental cyclic wear and tear. The cause of the separation between the skin and core of the immediate composite structure is not the main issue; the problem arises when the damage affects the structural integrity of the composite sandwich. These particular composite sandwich structures can range from spars and ribs in an aircraft to the haul of a boat. These applications are usually subjected to different loading conditions, which include compression to buckling loading. Introducing a mean of deterring delamination growth changes the structural behavior of the composite sandwich. The complexity of nonlinear foam and changes in moment of area affects the structural behavior of the composite sandwich, which makes analysis of the structure quite a challenge.

In this project, the components of the composite sandwich structure consist of fiberglass woven roving skin with a foam core being subjected to buckling load. The studies will look into the effects of ways to deter initial delamination from growing. This paper will discuss the experimental method, the effects of delamination arrestment key on initially delaminated composite sandwich under different test variables.

\subsubsection{Problems and Reparation of Delamination for Composite Sandwich Structure}

Face sheet delamination can be quite a tricky situation to deal with. The issue with delamination is that it demises the integrity and load bearing capacity of the composite sandwich structure but the problems with delamination go far deeper than the mechanical characteristics. The rehabilitation of structural damages on a composite sandwich structure, such as debond of the fiberglass skin from the foam core, can become complex during the structural repair procedure. Replacement of the structure is be a better choice than repairing the delaminated sandwich structure if the damage is too great or if the cost of structural repair supersedes the cost of replacing the component structure. 
If the structure is reparable, some basic methods used to repair delaminated composite sandwich structure include the following: patched repair, taper sanded or scarf repair, and step sanded repair [7]. The patched repair method can be done by first removing sections of the damaged plies, then adding composite plies to fill the damaged area, and finally adding plies to patch the damaged location. Figure 1 gives an example of the patched repair method.

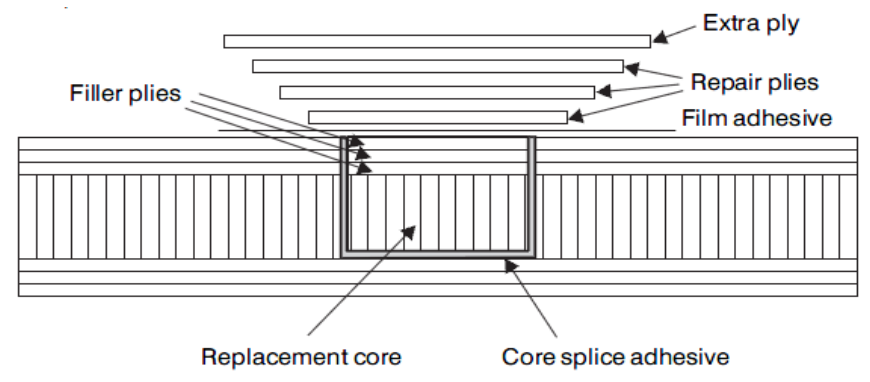

Figure 1: Patched Repair Method Example [7]

Similar to the patched repair method, the taper sanded or scarf repair method requires the removal of the damaged area and is sanded down to expose region of each ply in the composite skin. Then the composite structure is patched in a similar fashion as the patched repair method [5]. Figure 2 shows the taper regions and the patching method. 


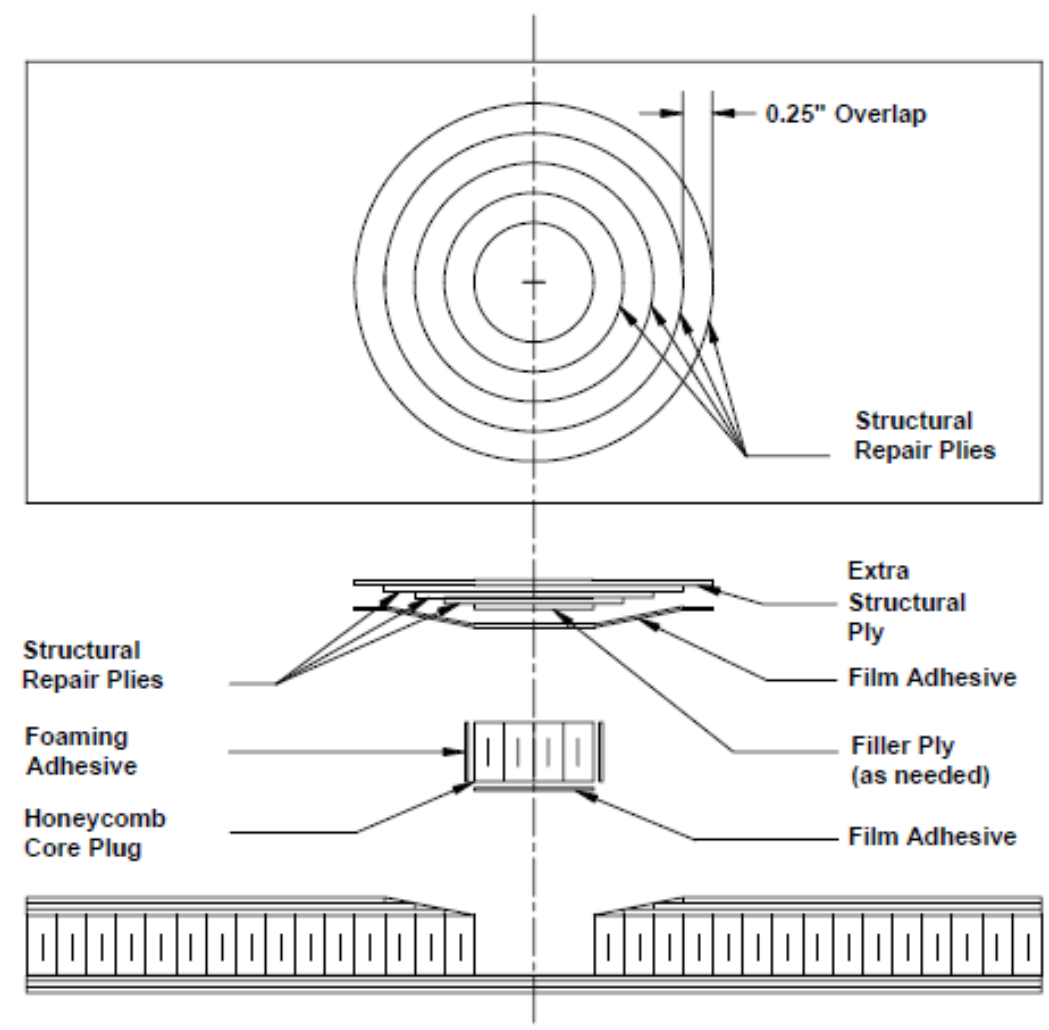

Figure 2: Taper Sanded or Scarf Repair Method Example [5]

The step sanded repair method is when the laminate is sanded until each ply is exposed in a stepped finish. The typical step lengths are approximately 1 to 2 inches per ply [7]. Once the stepped finish is complete, the patching of the damaged region is the same as the patched repair method. Figure 3 gives an example of the step sanded repair method.

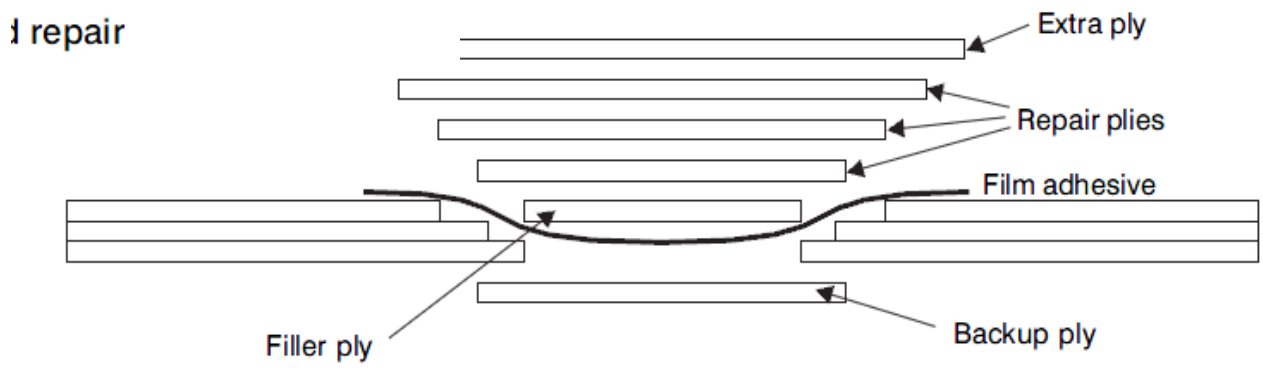

Figure 3: Step Sanded Repair Method [1] 
The major difference between the step sanded method and the scarf method is how well the repair ply bond and fit with the damaged region of the composite sandwich structure. Table 1 shows a summary of the various repair options and benefits.

Table 1: Delamination Reparation Options [7]

\begin{tabular}{|c|c|c|}
\hline Reparation Methods & Principal Advantages & Principal Disadvantages \\
\hline Patched Repair & $\begin{array}{c}\text { Requires minimum } \\
\text { preparation } \\
\text { Quick and simple to do }\end{array}$ & $\begin{array}{c}\text { A repaired laminate is thicker } \\
\text { and heavier than the original } \\
\text { Very careful surface } \\
\text { preparation is need for good } \\
\text { adhesion }\end{array}$ \\
\hline Taper Sanded or Scarf Repair & $\begin{array}{c}\text { Repair is only marginally } \\
\text { thicker than the original } \\
\text { Each repair ply overlaps the } \\
\text { ply that is repairing giving a } \\
\text { straighter, stronger load path }\end{array}$ & $\begin{array}{c}\text { Time consuming } \\
\text { High technical skill in } \\
\text { reparion needed and difficult } \\
\text { to achieve }\end{array}$ \\
\hline Step Sanded Repair & $\begin{array}{c}\text { Repair is only marginally } \\
\text { thicker than the original } \\
\text { Each repair ply overlaps the } \\
\text { ply that is repairing giving a } \\
\text { straighter, stronger load path }\end{array}$ & Extremely difficult to do \\
\hline
\end{tabular}

The idea of the three basic repair methods is quite similar to the repair methods used for metals, whereas a homogeneous metal doubler is applied to the damage region of the metal structure. The addition of a doubler to a metal structure maintains or exceeds the current load bearing capacity and transmits stresses across the repaired doubler region reducing the amount of applied stresses at the damaged region of a metal structure. This is also true for all three methods of composite repair and all of these methods can be completed using an autoclave or vacuum bag process. In most cases, patching is a way to prolong the life of the structure but this does not mean the structure will meet the initial design life. 


\subsubsection{Benefits of Delamination Arrest Keys}

Repairing a delaminated composite sandwich structure is quite troublesome and the methods of repair are limited in their techniques. The purposed idea of adding a delamination arrest mechanism into the composite sandwich removes the need for repair due to immature structural delamination. The proposed arrestment mechanism consists of a stiffener-like key that is integrated into the sandwich structure during fabrication. Even though this will increase the manufacturing time, it reduces the need for many future repairs.

Introducing a way to reduce the size of the delamination, or better yet, arrest the delamination from propagating in the composite sandwich structure can provide many benefits and advantages in the areas of:

1. Reliability

2. Durability

3. Safety

4. Economy

Reliability - The arrest mechanism in the composite sandwich specimen reduces, or even stops, the delamination from growing. The arrest mechanism is integrated into the structure during the manufacturing of the part to reduce the need for future integration. The arrest mechanism reduces the need for future rehabilitation of the composite structure caused by minor delaminated regions.

Durability - The integration of the arrest mechanism has a higher compressive loading capacity than the unimplemented composite sandwich structure due to the stiffness of the introduced delamination arrest key. The arrest mechanism will reduce or even elimination delamination growth under the study buckling loading. Predictions state that the higher loading capacity and reduction in delamination growth will provide a higher service life for the structure. 
Safety - The structural integrity of the composite structure determines its reliability and longevity. The arrest mechanism reduces the delamination growth and lowers safety risks. One major safety concerns is the human factor. If such a delamination was found on the skin structure of an airplane, the performance would decrease and could lead to issues with human safety. The increase in loading capacity provides a longer lifespan for the designed structural performance.

Economy - With a reduction or elimination of delamination growth, the need for repair or replacement of the composite structure will drop as well. This correlates to a reduction in repair or replacement cost. Maintaining the lightweight design reduces the time costs for transportation. The initial cost will likely be high in order to implement this, but the reliability, durability, and safety benefits would ultimately surpass the implementation cost and eventually decrease the overall cost.

The final product will improve the life of the composite sandwich structure by reducing, or even arresting, the delamination. The integration of the delamination arrest mechanism will see a slight increase in structural weight, but the arrestment mechanism also improves the strength properties of the existing composite sandwich structure by increasing its load bearing capacity.

\subsection{Thesis Objective}

There are quite a few studies on the effects of delaminated region on composite sandwich structure from how the face sheet will buckle to energy release under certain loading conditions. A factor that is well known with delaminated region is that the structure dramatically drops its loading capacity and losses its structural integrity. This statement stands true for laminates to composite sandwiches. There are also a few repair methods to resolve delaminated composite issue such as a patched, sanded, or even step sanded repair methods, but there exists a lack of investigative work on preventive maintenance. Prevent maintenance is used in terms of stopping the damage, in this case it would be a delaminated region, from initially occurring under different static and dynamic loading conditions. The idea is to introduce a delamination arrestment key into 
the composite sandwich structure as a way to reduce or stop delaminated regions from growing and increase the loading capacity of the overall composite sandwich structure. The arrestment key can be described as a semicircular rod with a length dependent on the composite sandwich configuration and is embedded between the skin and core.

The main objective is to experimentally investigate the effects of initially delaminated composite sandwich structures with and without the embedded delamination arrestment keys in several different configurations and under buckling loading. The different configurations include positioning the arrestment keys parallel and perpendicular to the in-plane load vector where the key is placed in a continuous or discontinuous arrangement. The materials used for the composite sandwich structure consist of fiberglass woven roving fabric, epoxy, and foam and wood cores. The wood core is used to strengthen the boundary of the structure for experimental testing. The materials used for the arrestment key are fiberglass strands and epoxy. However, several other factors must be investigated before the main objective can be achieved. These factors include examining the final solid curing duration of the epoxy with the fiberglass woven roving fabric, the weight and volume fractions of the composite sandwich structure and arrestment key, the geometric length per given cross-sectional area of the sandwich structure that will be a exhibit perfect buckling, and the effects of different initial delamination sizes.

The study starts off with the experimental design of the composite sandwich structure and develops procedures on how to manufacture these sandwich structures and on how to test the specimens. Several static test methods are used to determine the mechanical characteristics of composite sandwich structures and their individual components. The second part consists of experimental testing on the composite sandwich structures in order to determine their behavior and mechanical properties under buckling loading.

\subsection{Scope of Work}

The scope of work for this study is divided into multiple chapters for ease of understanding and reading. These chapters talk about the thesis project and its progression from 
preparing the design of the specimen to manufacturing to breaking the test specimens and analyzing the collected data. The chapters are as follow:

1. Introduction

2. Design of Experiment

3. Manufacturing, Prepping, and Testing Procedures

4. Theoretical Analysis

5. Experimental Results and Discussion

6. Conclusion

Introduction - The introduction of this thesis project is a hefty chapter that provides an overview of what are composite materials and what this project is all about. The chapter starts off with the 'motivation' section to see what drives the research, the problems that come up when dealing with composite sandwich structures, and the main objective of this thesis paper. Also, this leads to some more background information with literature review, previous and related work, and the characteristics and application of composite sandwich structures.

\section{Design of Experiment and Testing - The 'Design of Experiment and Testing' chapter} focuses on how the design was considered and chosen. The chapter provides a detail outlook of how the design consideration came to be and the evolution the designs went through. This chapter starts off by discussing what standards are currently being followed in industry and progresses from design considerations to how the test specimens and the experimental setup evolved over time. This is quite an important chapter because it discusses the many different variables considered in the design phase and why iteration of the design is appropriate.

Manufacturing, Prepping, and Testing Procedures - This is a transition chapter between the design and the test. The chapter discusses the manufacturing procedures that provide steps in how the test specimens will be manufactured and prepared for experimental testing. The chapter will go into detail about how the foam, delamination arrestment key, composite sandwich plate and specimen are prepared. This chapter will also discuss how the experiment is to be 
experimentally tested. This includes all the testing criteria for all the different test specimen configurations and testing for mechanical characteristic of each components of the composite sandwich structure.

Theoretical Analysis - This chapter looks into the theoretically yet ideal outcome of the composite sandwich structure. This includes looking into the weight and volumetric fraction analysis process.

Experimental Results and Discussions - The chapter is a breakdown of all the experimental results from testing. This includes the mechanical properties of the composite sandwich structure and its components, weight and volume fractions, geometric buckling length, final solid cure duration, and a comparison of all the configurations.

Conclusion - The conclusion sums up the entire paper and concludes the overall thesis investigation.

\subsection{Literature Review}

The literature review section of this chapter will look into the background of composite material, its application, leading all the way up to composite sandwich structure applications and issues. This section will also provide a summary of several research papers that pertains to this thesis project.

\subsubsection{Composite Materials and Applications}

Composite materials are quite a unique combination of two or more different materials that when put together form an improved material that meets the desired characteristics. The combination of the complementary materials usually results in a material that exhibits all the desired characteristics and eliminates the majority or even all undesired traits. Composite materials are everywhere and are used on a daily basis; some of which are so common that it is not recognized as composite engineering. A great example of this is concrete walls. The design of the concrete wall serves the purpose of holding the wall vertically, as high as possible, without having it tip over or collapse onto itself due to higher loads at the lower portion of the wall. This 
is done using rebar to carry the load and distribute the load across the over structure and the cement as the matrix material that holds the rebar in the specific positioning. Nowadays, advanced composites are using fibrous reinforcement and epoxy matrix materials to offer highly desirable characteristics such as high strength-to-weight ratio, variable conductivity, and thermal indifference.

These advanced composite materials are progressively taking the roles of many metals previously used on automobiles, aircrafts, and many different industries. Even with the high structural performance specifications, composite materials are controllable in design to the point that it can provide the optimal performance on the material level. Various method of controllability includes the orientation of the fibers in the fibrous reinforcement to the amount of epoxy in the material. This freedom for optimal design and performance of composite materials is quite an evolutionary leap from structural optimization down to the material level. Several different examples of composite use can be seen in Figure 4.

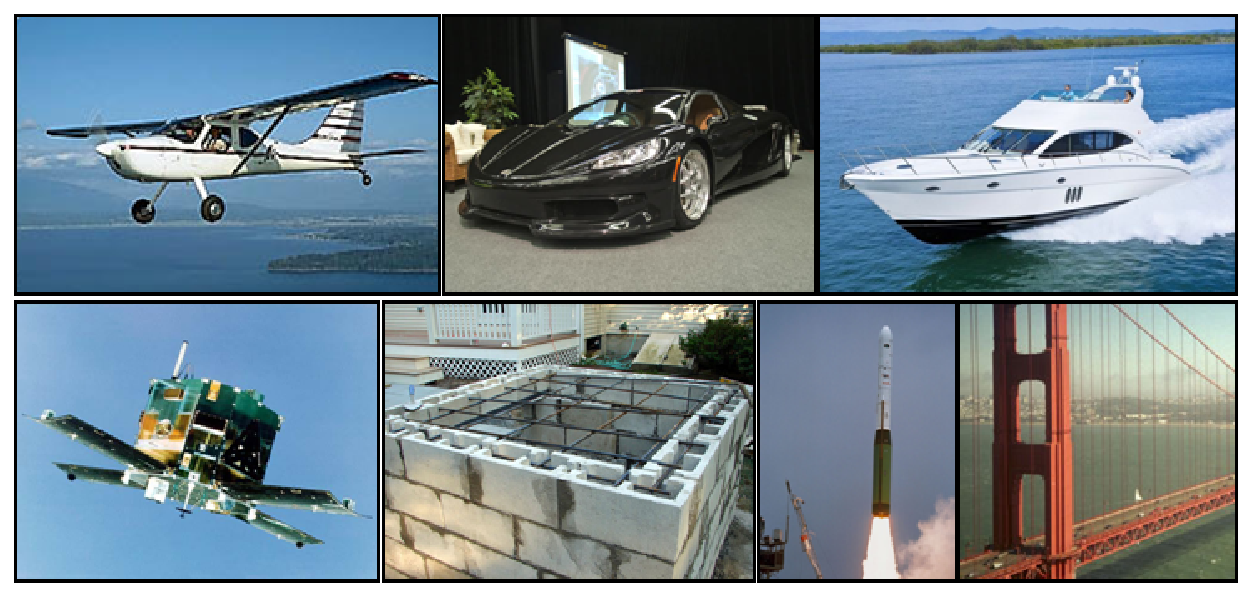

Figure 4: Advanced Composite Material Uses Example

\subsubsection{Composite Characteristics}

Composites can be divided up into two specific material categories: the reinforcement and the matrix material groups. The reinforcement is usually defined by its ability to carry load and it usually has a higher strength and stiffness than the matrix material. The matrix material is 
used to hold and position the reinforcement material in a certain fashion and used to transfer load since the reinforcement material can be discontinuous in nature while the matrix materials are continuous in nature. Load bearing properties of the composite are usually provided by the reinforcement, but it is the combination of the reinforcement and matrix that provides a harmony between all the properties of the constituent materials that makes the composite. The mechanical characteristics of the composite cannot only be summed up by the volume of reinforcement and matrix, but also requires geometric properties. Some of these geometric properties include the shape, numbers of plies, and ply orientation.

Composite materials are quite flexible in how they are designed and optimized to the specific requirements for the job at hand. Composite materials are usually defined by advances in its mechanical properties such as the strength, stiffness, and durability. Other variables that are occasionally used include the electric and thermal conductivity of the composite material and also its resistance to corrosion and damages.

\subsubsection{Disadvantages of Composite Materials}

Much has been discussed about the advantages of composites in Section 1.3, but there are also disadvantages with composite materials. The three big disadvantages to composite materials are higher manufacturing costs, significant drop in performance when damaged, and maintenance. The cost for composite materials is generally higher than common materials used such as metals and plastics. In instances where composite materials comprise a majority of the structure such as on a Boeing 787, the cost is higher upfront but has more savings in the long run. The high cost is due to material cost and manufacturing while the saving comes from the lightweight characteristic. Another disadvantage is that damages to the composite structure can dramatically decrease its performance. The main reason is that the damage creates a discontinuous region that does not allow for the load to transfer and experience higher stresses. Deterioration of the structural integrity of the composite structure can be caused by a minor hairline crack that can 
propagate between the laminas to even impact damage from foreign objects. This is a reason why composite materials require higher level of care and inspection. If there is damage, maintenance or repair on the composite structure is required. This is usually done by removing the discrepant materials from the damaged area and patching it up with new materials. An issue with such a repair is that the patched area creates an eccentric stress concentration and affects the longevity and performance of the composite structure.

\subsubsection{Fibrous and Particulate Composites}

There are many different ways of create composite materials because of its flexible characteristics for manufacturing. Three composite themes that are usually seen in designing the composite structures are the use of continuous fiber, discontinuous or short fibers, and particles. These three composite designs each have different features and performances. The continuous fiber is defined by its geometry where the length is greater than the cross-sectional dimensions; it does have any discontinuous points through the composite structure, and is usually from one boundary end to the other. On the other hand, the discontinuous or short fiber does have breaks in the composite structure and the fibers orientation is difficult to account for. The particulate composite design geometry has approximately the same dimensions in all directions. Figure 5 displayed what these three composite types and its major differences [6].
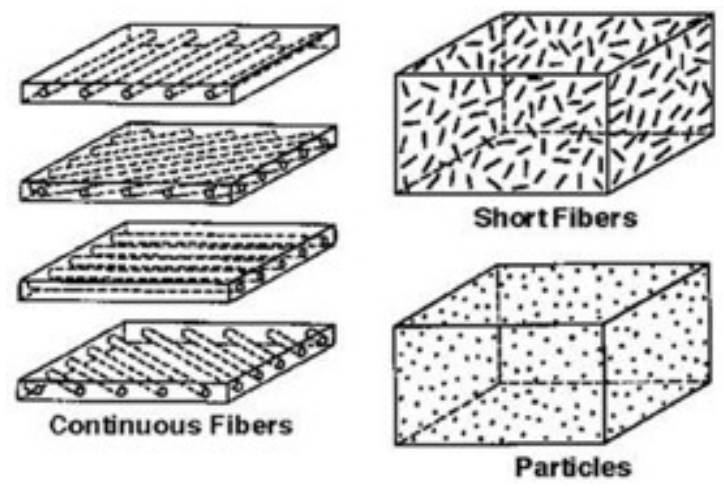

Figure 5: Fibrous and Particulate Composites [6] 
The importance of fibrous composite materials is represented in how effective the fibers are carrying and transferring the loads through a composite structure and its resistivity in matrix failure. Fibrous composites are quite different from something of a homogenous, isotropic nature such as metals and certain plastics because the mechanical properties of such a structure changes with directions. In comparison with such isotropic materials, fibrous composite structure exhibits most of its strength in the direction of the fibers.

Continuous fibrous composite is separated into its own category where there are no breaks in the fibers usually from one boundary end to the other. Continuous fibrous composite does consist of quite a few long composite fibers bundled up and usually oriented in one to two different directions. These 'unidirectional' and 'bidirectional' oriented fibers are both defined by their orthotropic traits. As discussed earlier, orthotropic materials have different material properties in different directions. This is why it is commonly known that strength is associated with the orientation of the composite structure. For example, the highest strength of a unidirectional fiberglass structure is in the direction of the fiber and the lowest is perpendicular to that fiber direction. The epoxy is a weaker material in nature but plays an important role by binding the fibers together and helping in distributing the loads through the fibers.

Like the unidirectional fiberglass fabric, there are many other types of woven pattern of the fabric. A great example of this is a bidirectional fiberglass woven roving. The fiberglass strands in the bidirectional woven roving are weaved in between each other to form great reinforcement and are great for carrying loads. Figure 6 shows examples of the different types of continuous fiberglass materials [17-19]. There are many more patterns than what is shown in Figure 6 and each of them provide unique material characteristics due to their geometry and pattern. 


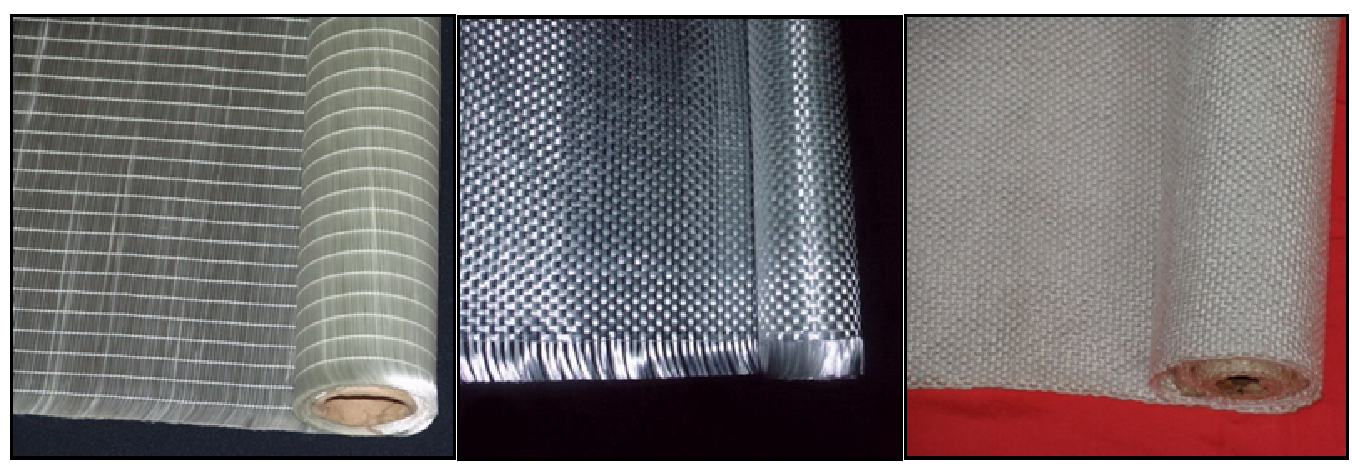

Figure 6: Examples of Continuous Fiberglass Materials [17-19]

These continuous fibrous materials such as the woven roving provide great performance and are very diverse in their use. The continuous fiberglass material is also quite easy to manufacture large structure in large quantities and at relatively low cost. The material characteristics of the fiberglass reinforced structure add great attributes to many different aerospace systems such as low weight, significant strength and rigidity, resistivity to corrosion, and impact resistance depending on the pattern. The continuous fiberglass reinforced material is applied in such places as the wings of the plane, the interiors, and even the walls of satellites. Figure 7 shows examples of the applications of the continuous fibers [1,20].

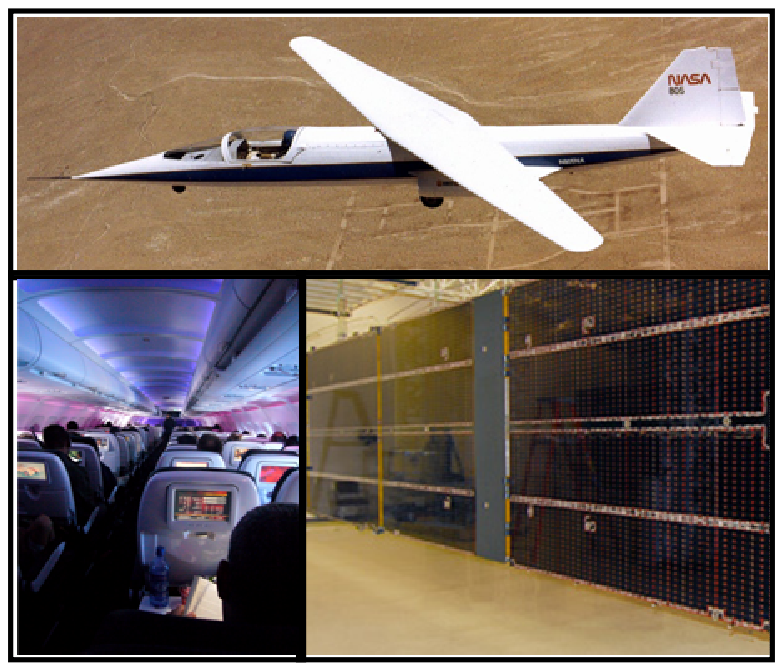

Figure 7: Examples of Application of Continuous Fiber Materials [1,20]

The short, or discontinuous, fibrous composite consists of fibers that are shorter and does not extend the length of the structure. The maximum length is approximately a few centimeters 
long. Rather the shortness of the fibers results in fibers pointing in many different and arbitrary directions. The fibers are still taking the loads but require more of the matrix material to transfer more of the loads across the overall composite structure and through the fibers. This type of fibrous composite is the immediate state between continuous and particulate composite types. The strength and stiffness of this composite structure is much weaker than the continuous fiber and is anisotropic.

The particulate composite material is where the dimensions of the particles are approximately the same in all directions. The main purpose of particulate composite, also known as particulate reinforcement, is to act as doping materials that helps increase the strength and helps prolongs the failure of a given structure. The idea is to provide more points where the loads can be carried and transferred across the structure. In addition, several other attributes include increasing the bond between mating materials and the ability to maintain the lightweight of the composite structure. Such materials used in composite structures are nanospheres and microballoons. These particular materials are usually infused into the composite structure when the resin infusion process is used. Particulate material is not used in this thesis project, but can be applied to future works to see its impacts.

\subsubsection{Application of Composite Sandwich Structures}

Composite materials such as carbon fiber and fiberglass are also extended to forming composite sandwich structures. The idea of composite structure consists of the composite skin and a compatible core. Composite sandwich structure provides an increase in geometry over simple laminate and more complex and possibly nonlinear mechanical properties. Figure 8 displays a diagram of the composite sandwich structure. 


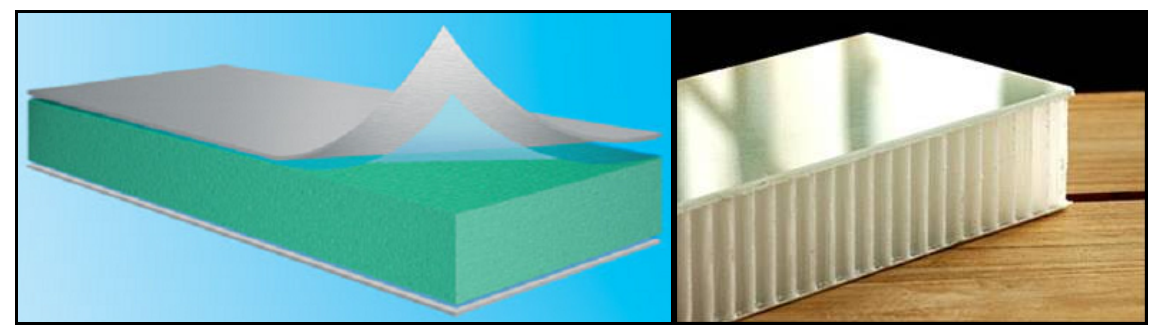

Figure 8: Composite Sandwich Structure [21-22]

Great examples of its application are found in the boating and aerospace industry. The most common types of cores used in these industries are honeycomb and foam. The foam core is usually designed for use in the haul of a boat due to the fact it's lightweight, provides great buoyancy, and is an insulator. The core is mated to composite materials such as the fiberglass woven roving as discussed earlier and provides a significant change in the strength and resistance of the composite structure. Fiberglass woven roving skin and a foam core is used to create the test specimen for this thesis project. A honeycomb core is usually seen in the aerospace industry because it is able to save more weight than the foam core. With planes and satellites, the cost per structural weight is a major issue that is highly sought after. The honeycomb core is used in many locations on a plane such as the spars in the wings to struts in the fuselage. Examples of these applications are in Figure 9.

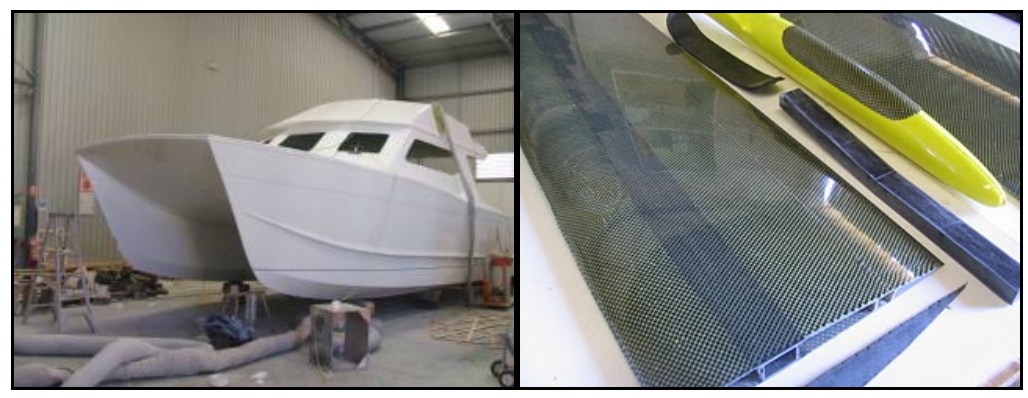

Figure 9: Application of Composite Sandwich Structures

\subsubsection{Problems with Composite Sandwich Structures}

Like any engineered structures, there are limitations and issues that can come up. One of these is delamination where the bond between the skin and core is broken and there is a separate between the two mating materials. The issue with delamination is quite a frequent one and usually 
caused by damages, improper manufacturing, or contaminates. This failure dramatically impacts the structural integrity and the delamination can propagate if left untreated. The issue will be studied in this project to see if the delamination can be deterred while maintaining the integrity of the composite sandwich structure. Examples of the delamination issue are displayed in Figure 10.

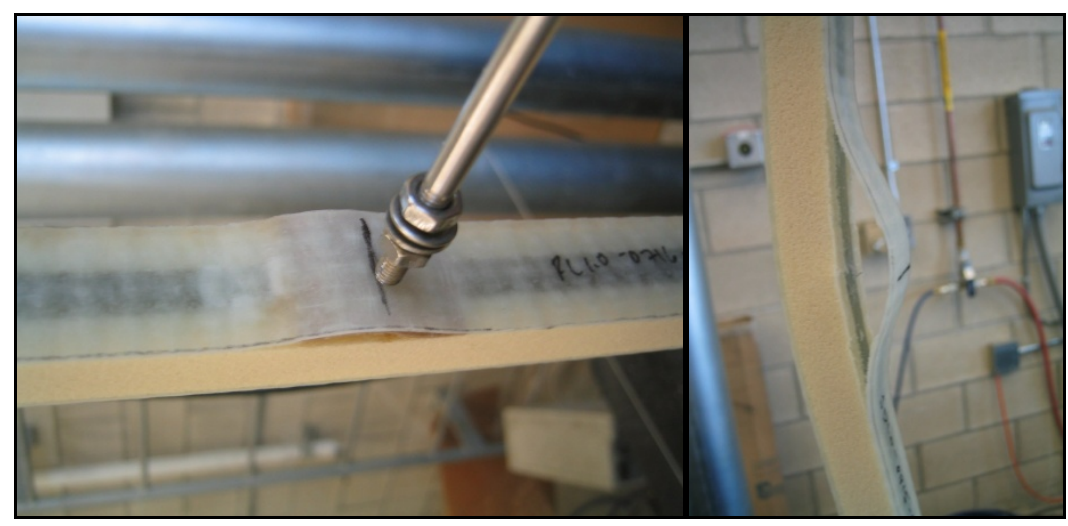

Figure 10: Delamination Issue Examples

Other problems with composite sandwich structures include maintenance of damaged structures, weight increase, and concerns about the operation ranges. The cost for maintenance or repair of composite sandwich structure is a bit higher than common material such as metals and plastics. The different types of repairs are discussed in Section 1.1.1. Depending on the required repair, it can be difficult to do and time consuming. It also creates an eccentric stress concentration at the region location, which means it may require more material to stiffen the location. The weight increase is a trade off for the increase in structural strength. The operation limits are more complex than a typical composite laminate; thermal deformation, corrosion, and electrical conductivity are some factors that have to be considered for all the different material use.

\subsubsection{Previous and Related Works}

This section of the first chapter will discuss several research papers that pertain to this thesis project.

\subsubsection{Tran et al.}


This senior project was completed by Tran et al. at California Polytechnic State University at San Luis Obispo, California. The project was conducted to investigate the effects of monotonic buckling on fiberglass and foam composite sandwich specimens with an initial delaminated region with and without embedded arrestment key perpendicular to the in-plane load vector. These monotonic experiments were conducted under various temperatures to also see the thermal impacts.

Each of the specimens consists of the same constant cross-sectional area. The delaminated composite specimens consisted of a DIAB H100 PVC foam core with two sets of alternating layers of chopped strand mat and $18 \mathrm{oz}$ fiberglass woven roving from Jamestown Distributors of each side. The epoxy used is from West Systems 105/206.Example of the materials is displayed in Figure 11. Also note that the PVC foam core is 0.5 inch thick.

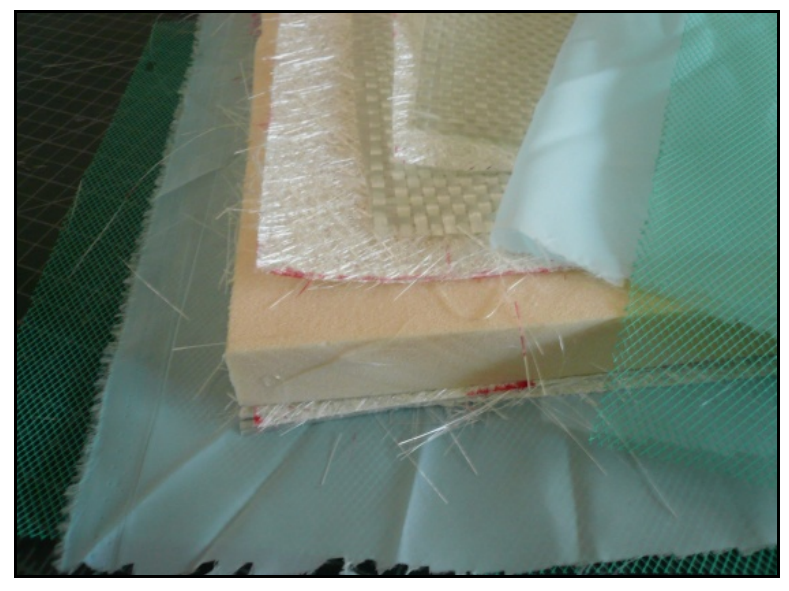

Figure 11: Examples of Materials used in Project by Tran et al. [25]

The delaminated composite sandwich structures consist of a delamination at the center which varies in length approximately 1 to 2 inches. These test specimens are embedded with two arrestment keys each and the keys has a similar semicircular cross-sectional area, but has a larger diameter. The same principle still applies for this project by Tran et al., which is used to help deter the delamination growth and maintain the loading capabilities. 
The arrestment keys are placed in offset distances from the delaminated region. All of the specimens were manufactured using a vacuum resin infusion (VRI) technique. Figure 12 shows an example of a part that is vacuumed and ready for resin infusion.

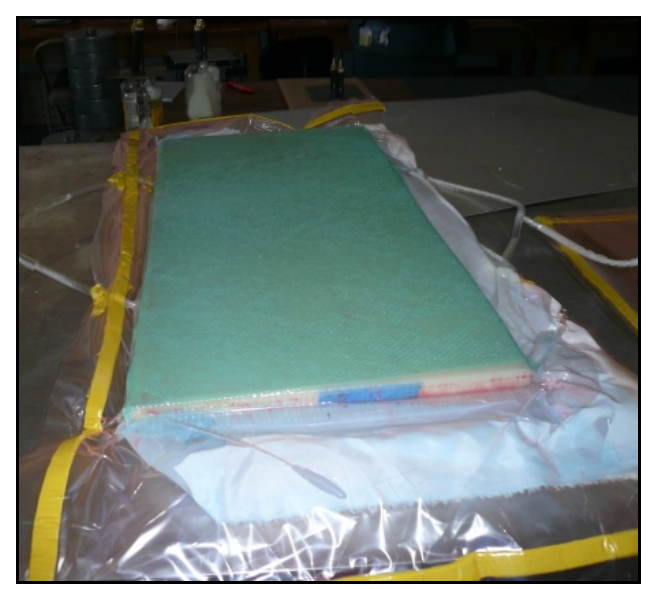

Figure 12: Example of VRI Part [25]

The introduction of the delaminated region into the test specimens required the use of waxed non-porous strip spanning the delamination length and manufactured at the same time as the rest of the composite sandwich structure. To manufacture the arrestment keys and obtain the chosen cross-section shape, the manufacturing process required a milled female mold where the fiberglass strands are placed into its grooves and vacuum resin infused. A set of pictures of the mold and how the fiberglass strands are placed in displayed in Figure 13.

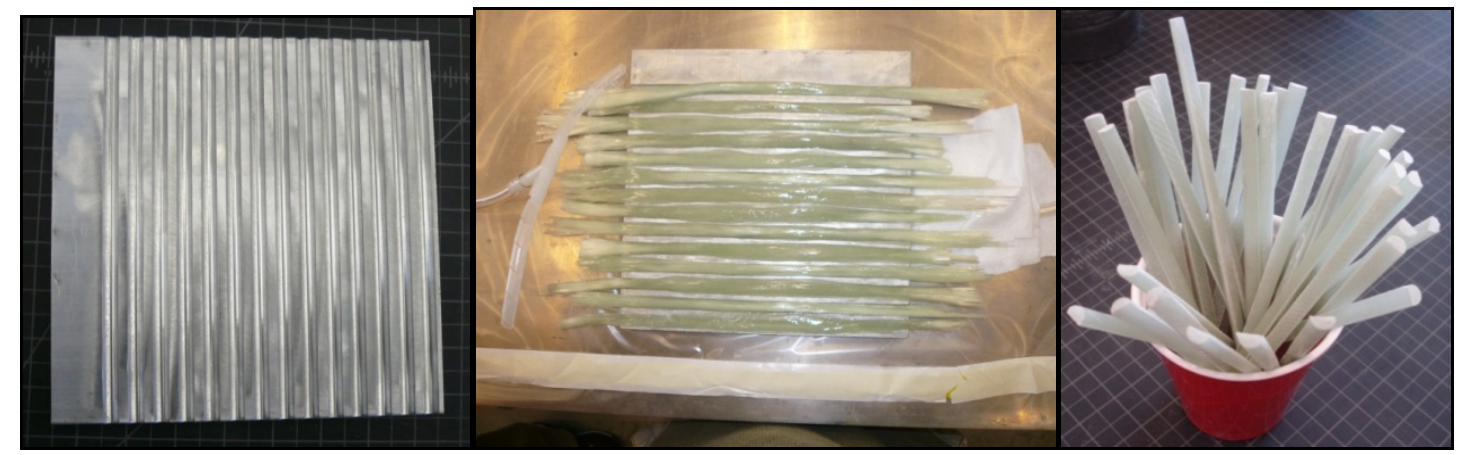

Figure 13: Mold and Fiberglass Strand Layup for Arrestment Keys [25]

Secondly, the PVC foam core had to also be milled to form the grooves for embedment of the arrestment keys. The various delamination lengths that were studied are 0 to 2 inches per 
one inch increments respectively; the variation of the shear key specimens varies from 0 to $5 \mathrm{~mm}$ on either side of the delamination. The specimens are analyzed using a constant displacement rate of $0.5 \mathrm{~mm}$ per minute at room temperature, $135^{\circ} \mathrm{F}$, and at $200^{\circ} \mathrm{F}$.

The experimental results reveal that the thermal differences have a significant effect on the mechanical properties of the test specimen. The results show a reduction in the critical load by as much as 70 percent for $135^{\circ} \mathrm{F}$ compared to room temperature and up to 86 percent for $200^{\circ} \mathrm{F}$ compared to room temperature. Introducing a 1-inchdelamination lowers the maximum critical load by 53 percent and a 2-inchdelamination decreases the critical load a bit more. The embedded keys placed perpendicular to the in-place load vector did not significantly increase the strength of the test specimens. The introduction of arrestment keys did increase the maximum critical load by 3 percent. The thesis project is an iteration of this project where it investigates the difference configurations.

\subsubsection{Goswami et al.}

The report by Goswami et al. studies the effects of delamination between the face sheet and core in a sandwich structure under transverse loading. The work investigates the crack propagation of predisposition delamination issues due to the transverse loading. According to Goswami et al., the presumed transverse loading does not buckle the delaminated face sheet but promotes crack propagation. The study uses a simulation of a sandwich structure using finite element modeling to determine the energy release rate based on the material and geometry.

The cause for delamination is usually due to imperfection in the manufacturing process according to Goswami's paper and details a significant drop in loading capabilities when there is a delamination in the composite sandwich structure. The most common failure with delamination is that buckling then collapse of the face sheet from the core material. The paper states that transverse loading is when the load is applied in the normal to the sandwich plane and the main purpose of composite sandwich structure is to carry this type of loading [26]. 
In order to study the effects of the delamination crack with the transverse loading conditions, the finite element model of the composite sandwich structure had to have twice the length and a delamination crack of $2 a$, where ' $a$ ' is the crack length. The crack location of the model is subjected to mode II loading, which details shearing stresses at the crack tip location. This allows for crack propagation growth. A diagram of this is detailed in Figure 14 with the transverse loading conditions and Figure 15 displays how the delamination is extended when the loading is applied.

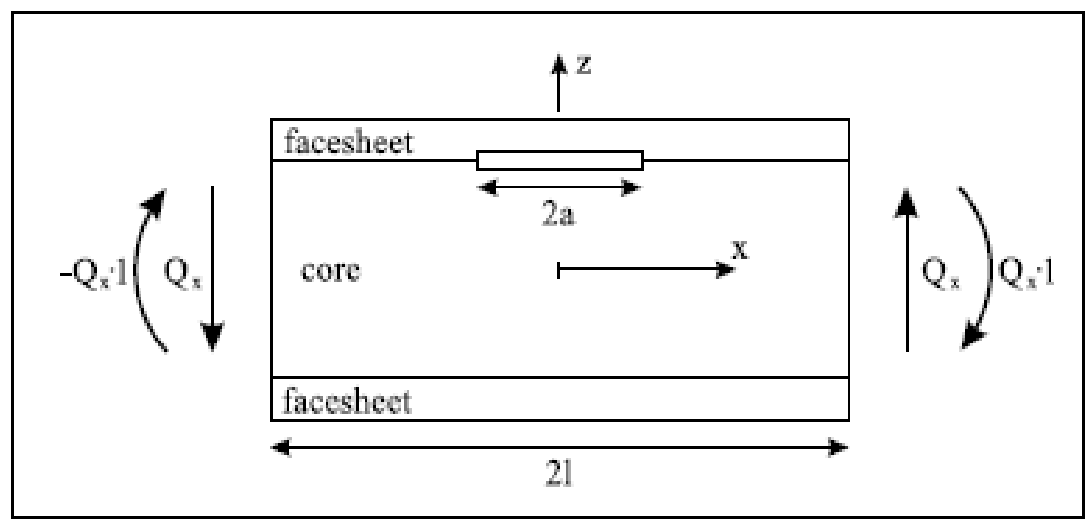

Figure 14: Debond Crack with Transverse Loads

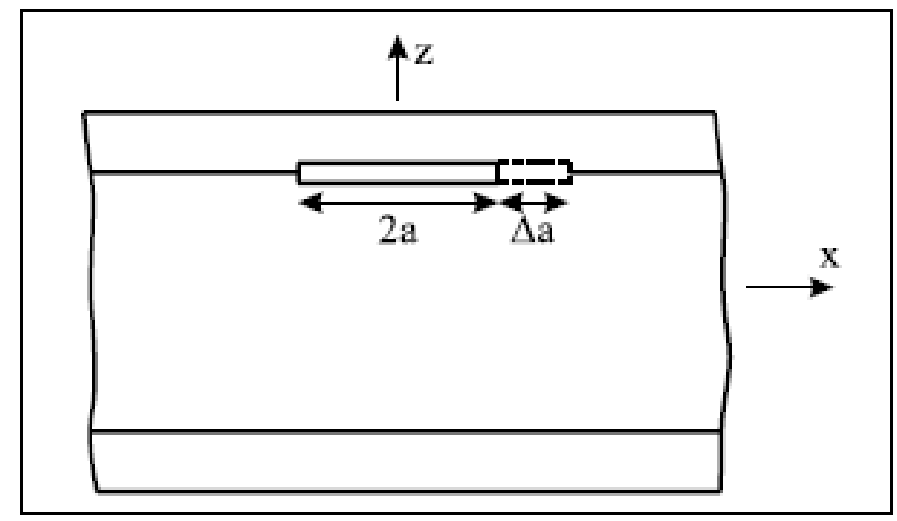

Figure 15: Crack Propagation when Loaded

The crack growth was studied using Irwin's crack closure integral approach. Irwin's approach to crack propagation can be used to determine the energy released which is equal to the 
amount of work required to close the initial $2 \mathrm{a}$ crack length. The parameters including the dimensions and material properties used for this project are displayed in the following table:

Table 2: Dimensions and Material Properties for Goswami et al. [26]

\begin{tabular}{|c|c|c|}
\hline Parameter & Units & Values \\
\hline Plate Length & $\mathrm{mm}$ & 200 \\
\hline Core Thickness & $\mathrm{mm}$ & 20 \\
\hline Face Sheet Thickness & $\mathrm{mm}$ & 4 \\
\hline Total Sandwich Thickness & $\mathrm{mm}$ & 28 \\
\hline Face Sheet Orientation & $\mathrm{deg}$ & {$[0 / 90] \mathrm{s}$} \\
\hline E1 & $\mathrm{MPa}$ & 135000 \\
\hline E2 & $\mathrm{MPa}$ & 10000 \\
\hline E3 & $\mathrm{MPa}$ & 10000 \\
\hline G23 & $\mathrm{MPa}$ & 3972 \\
\hline G13 & $\mathrm{MPa}$ & 5000 \\
\hline G12 & $\mathrm{MPa}$ & 5000 \\
\hline$v 23, v 12, v 13$ & - & 0.27 \\
\hline E of foam & $\mathrm{MPa}$ & 100 \\
\hline$v$ of foam & - & 0.33 \\
\hline
\end{tabular}

These parameters were used to define the finite element model and the model was meshed with 7524 nodes, 7056 elements, and 15046 degrees of freedom. This is quite an extensive amount of elements and nodes, but it will provide a better understanding of the behavior of the composite sandwich model.

The studied sandwich model has been subjected to the equilibrium loading conditions displayed in Figure $\mathbf{1 4}$ where the transverse load is loaded 5 Newton per millimeter, the applied moment is 500 Newton-millimeter in the $\mathrm{x}$ direction, and variable crack length from 0 to $30 \mathrm{~mm}$. The resultants show an increase of energy release rate per increase of crack length. Figure 16 displays these results [26]. 


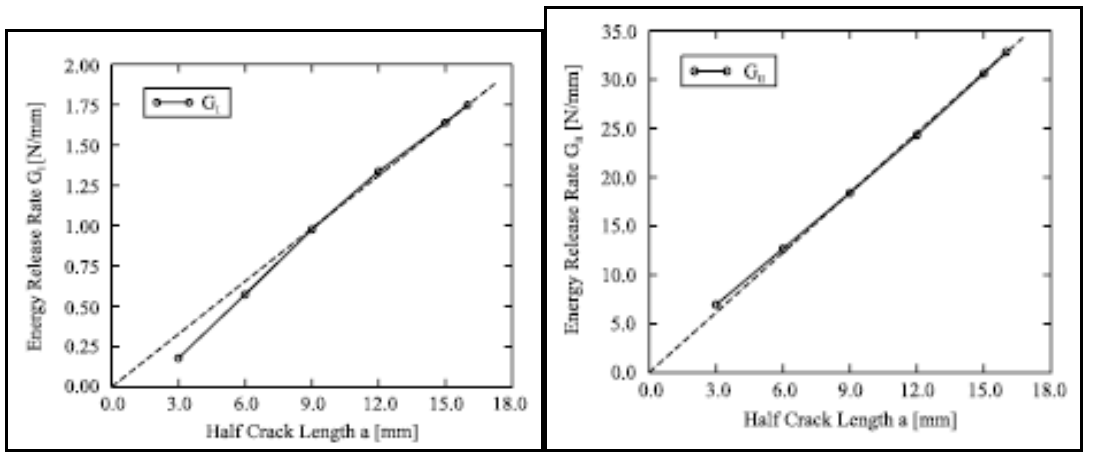

Figure 16: Variation of Energy Release Rates [26]

In this paper, there are also other variations that were considered such as the contact or no contact of the elements, change in energy release rate with various elastic moduli and face sheet thicknesses.

\subsubsection{Hwang et al.}

Hwang et al. is another research paper dealing with the delamination crack growth issue.

This particular paper studies the responses of buckling and post buckling of unidirectional composite materials with two delaminations under compressive loading. The strain energy release rates were investigated around the crack tip where the delaminations were placed in different locations and with different length [27]. From the introduction of this paper, the buckling mode responses were first discussed. There are two different responses of buckling modes: local, mixed, and global. Figure 17 shows the different types of buckling mode responses. Part (a) is the local buckling mode, part (b) is the mixed buckling mode, and (c) is global buckling response.

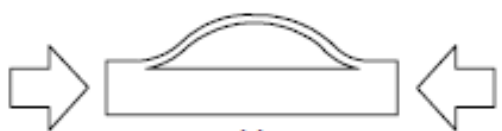

(a)
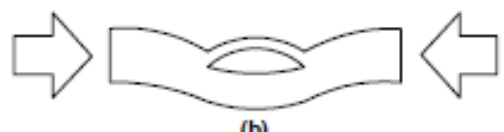

(b)

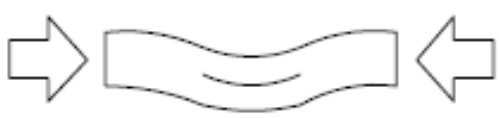

(c)

Figure 17: Buckling Mode Responses 
The project studied one particular type of design for their composite structure. The specimen that was used consists of 16 carbon and epoxy layers. The mechanical characteristics are listed in the table below:

Table 3: Mechanical Characteristics for Carbon/Epoxy Composites

\begin{tabular}{|c|c|c|}
\hline Variable & Unit & Value \\
\hline $\mathrm{E} 11$ & $\mathrm{GPa}$ & 121 \\
\hline $\mathrm{E} 22$ & $\mathrm{GPa}$ & 9.37 \\
\hline $\mathrm{G} 12$ & $\mathrm{GPa}$ & 6.25 \\
\hline $\mathrm{v} 12$ & - & 0.23 \\
\hline Fiber Volume & - & 0.6 \\
\hline
\end{tabular}

From their research, the resultant data shows that the buckling response was little to none for short delamination length. The longer the delamination length, the buckling mode became more visible. The transition followed the three types of buckling mode response with increase in the delamination length. Hwang et al. studied starts off by stating that the delamination length to the specimen gauge length is defined by a/L ratio. The ratios that were used are $0.3,0.5$, and 0.7 . The other ratio is the short delamination to the long delamination length, which is denoted by $a_{s} / a$ and ranges from 0.1 to 1 . The other factor that was considered is the two different types where Type A scenario has the inner delamination being less than the outer delamination and Type B is the opposite of Type A.

For Type A, Hwang et al. found that the short delamination had no effect or changes in comparison to their control specimen which had only one delamination. This differs for Type B scenario though, the outer delamination showed buckling stresses and the critical value are much larger. Diagrams of this are shown below in Figure 18 and Figure 19. 


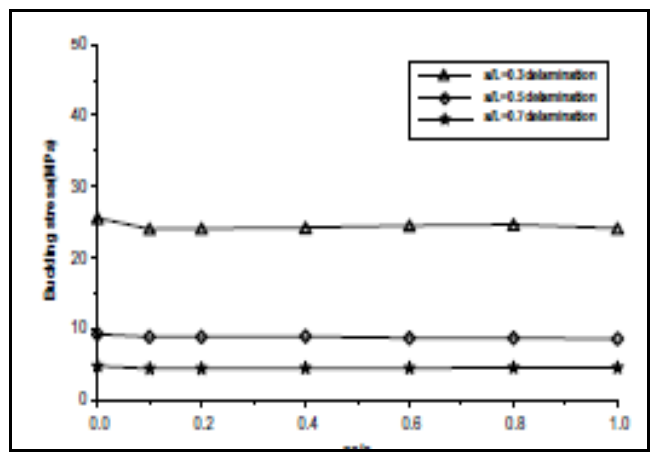

Figure 18: Buckling Stresses of Type A

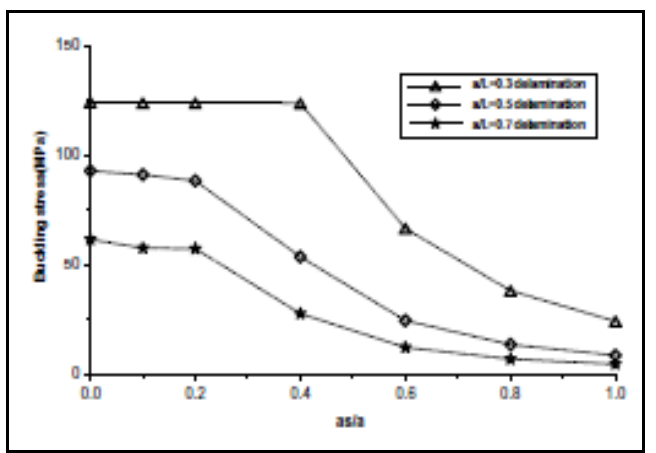

Figure 19: Buckling Stresses of Type B

Type A scenario shows a transition from the local buckling mode to mixed mode because the external compression stress is larger than the buckling stress [27] and Type B scenario shows a significant difference where the short delamination must be taken into consideration. The long delamination in Type B overwhelms the short delamination which causes the inner delamination to grow under mixed fracture mode. It is important to note that the geometry and the two delaminations affect the results significantly. Type A does not show much of a change while Type B shows a drop in the buckling stresses per delamination sizes. 


\section{DESIGN OF EXPERIMENT}

\section{$2.1 \quad$ Testing Standards}

There are several ASTM standards used and referenced for the various different experimental tests to acquire the desired material properties. The specific ASTM standards that were considered are:

1. ASTM D-3039

2. ASTM D-3410

3. ASTM D-3171

4. ASTM C-364

More details regarding these particular testing standards are described in the sections below.

\subsubsection{ASTM D-3039 Standard Test Method for Tensile Material Properties}

After some preliminary experimental testing, there was an issue found regarding the curing cycle of the epoxy used to form the composite sandwich structure. The specimen was manufactured using vacuum resin infusion at room temperature which required a longer period of time to fully cure than expected. The ASTM D-3039 standard was used to find the tensile material properties of the specific fiberglass skin portion of the composite sandwich structure [3]. Those material properties consist of the load, extension, stress, strain, and tensile elastic modulus of the material in order to determine if there are any changes over the course of 15 days. More details about this particular issue will be detailed later in this chapter. In order to achieve the standardized test results, the design required by the ASTM D-3039 standard is detailed in Figure 20. 


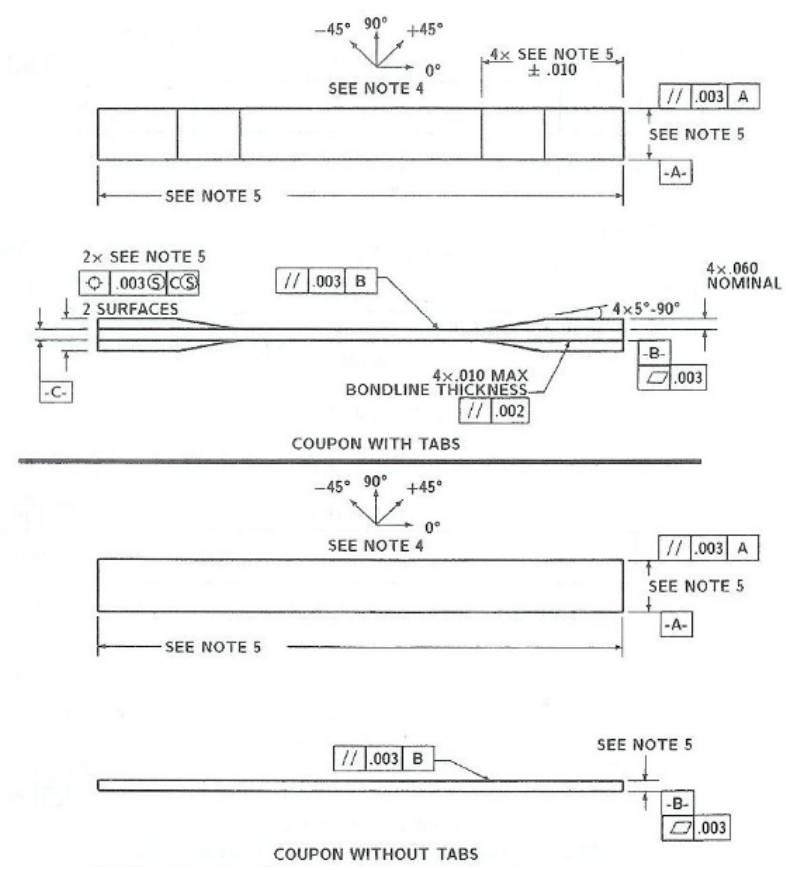

Figure 20: Design Requirements of ASTM D-3039 [3]

The design requirement also provides recommended geometry used to create the tensile specimen. Table 4 details those dimensions:

Table 4: ASTM D-3039 Recommended Geometry [3]

\begin{tabular}{|c|c|c|c|c|c|}
\hline $\begin{array}{c}\text { Fiber } \\
\text { Orientation }\end{array}$ & $\begin{array}{c}\text { Width } \\
\text { (in) }\end{array}$ & $\begin{array}{c}\text { Overall } \\
\text { Length (in) }\end{array}$ & $\begin{array}{c}\text { Thickness } \\
\text { (in) }\end{array}$ & $\begin{array}{c}\text { Tab Length } \\
\text { (in) }\end{array}$ & $\begin{array}{c}\text { Tab Thickness } \\
\text { (in) }\end{array}$ \\
\hline $\begin{array}{c}\text { 0 deg } \\
\text { unidirectional }\end{array}$ & 0.5 & 10.0 & 0.040 & 2.25 & 0.062 \\
\hline $\begin{array}{c}90 \mathrm{deg} \\
\text { unidirectional }\end{array}$ & 1.0 & 7.0 & 0.080 & 1.0 & 0.062 \\
\hline $\begin{array}{c}\text { Balanced and } \\
\text { symmetric }\end{array}$ & 1.0 & 10.0 & 0.100 & Emery cloth & - \\
\hline $\begin{array}{c}\text { Random- } \\
\text { discontinuous }\end{array}$ & 1.0 & 10.0 & 0.100 & Emery cloth & - \\
\hline
\end{tabular}

The recommended geometry was used to manufacture the specimens required for testing. Approximately 5 to 6 specimens were made to test per each testing day. Once the test fiberglassepoxy test specimens were made, the specimens were then tested per workmanship requirements in compliance with ASTM D-3039. The following workmanship requirements are applied to determine the material properties of the cut down and prepared fiberglass-epoxy test specimens. 
Rate-Controlled Tests - It is important to regulate the speed of testing in order to reduce error from increased speed and provide a better strain response versus time. It is found that with increased head displacement rate, there is a higher standard deviation between multiple test specimens per same design requirements. A constant displacement rate of $0 . f 5$ inch per minute is the quasi-static rate used by ASTM D-3039 standard. The suggested strain rate is $0.01 \mathrm{~min}^{-1}$ which provides a slow rate in order to reduce any inappropriate failures.

Test Environment - It is quite important to know the moisture and temperature profile for the test specimens. Elevated temperatures and humidity affecting the specimens can provide unrealistic results and fall out of the testing profile. The temperature and moisture levels are recorded per testing time. All test specimens should be tested within the same testing profile in order to eliminate any errors caused by the fluctuation in temperature and moisture levels. The test environment in Composite/Structure Lab at Cal Poly, San Luis Obispo is limited in managing the temperature and moisture, thus the test specimens are tested under a wider testing profile.

\subsubsection{ASTM D-3410 Standard Test Method for Compressive Properties of Composite} Materials

ASTM D-3410 test method is used to find the compressive properties of composite materials. These properties include finding the compressive elastic modulus, strength, strain, and Poisson's ratio of the test specimens. In this particular case, the material studied is a composite strip consisting of $18 \mathrm{oz}$ fiberglass woven roving and West Systems 105/206 epoxy.

The fiberglass plate is cut down to flat strips with the guidance of the design requirement and recommended dimensions found in the ASTM D-3410 standard test method procedure [4].

The design requirement recommended by the standard test method is to create a rectangular shape test specimen to provide a constant cross-sectional area when the specimen is applied under compression loading. The recommended design requirement is shown in Figure 21. 

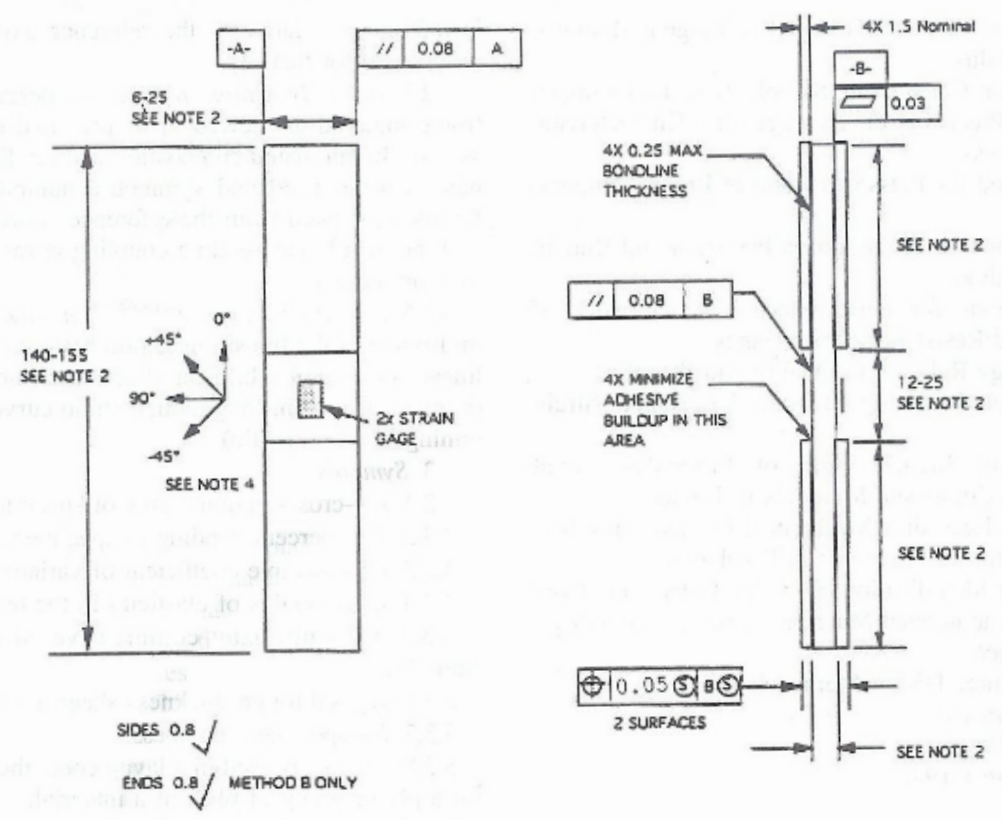

Figure 21: ASTM D-3410 Design Requirement

Similar to the ASTM D-3039 design requirement, the test specimen is fashioned in this specified way expect for length of test section. ASTM D-3410 standard test method requires a smaller test section to avoid unstable behaviors from occurring, which are found in larger gage area such as bending and buckling. The ideal short length is used to determine compressive material properties without dealing with unwanted factors. The compression specimen geometries recommended by ASTM D-3410 are: 
Table 5: ASTM D-3410 Recommended Geometry [4]

\begin{tabular}{|c|c|c|c|c|c|}
\hline $\begin{array}{c}\text { Fiber } \\
\text { Orientation }\end{array}$ & $\begin{array}{c}\text { Width } \\
\text { (in) }\end{array}$ & $\begin{array}{c}\text { Gage Length } \\
\text { (in) }\end{array}$ & $\begin{array}{c}\text { Tab Length } \\
\text { (in) }\end{array}$ & $\begin{array}{c}\text { Overall } \\
\text { Length } \\
\text { (in) }\end{array}$ & $\begin{array}{c}\text { Tab Thickness } \\
\text { (in) }\end{array}$ \\
\hline $\begin{array}{c}\text { 0 degrees } \\
\text { unidirectional }\end{array}$ & 0.5 & $0.5-1.0$ & 2.5 & $5.5-6.0$ & 0.06 \\
\hline $\begin{array}{c}90 \text { degrees } \\
\text { unidirectional }\end{array}$ & 1.0 & $0.5-1.0$ & 2.5 & $5.5-6.0$ & 0.06 \\
\hline $\begin{array}{c}\text { Specially } \\
\text { Orthotropic }\end{array}$ & 1.0 & $0.5-1.0$ & 2.5 & $5.5-6.0$ & 0.06 \\
\hline
\end{tabular}

The tabs are placed on the specimen in the same exact fashion as found in Section 2.1.1.

The tabs, similar to ASTM D-3039, will be carrying the shearing load but constrained by the applied loading from the wedge grips [4]. The wedge grip design from ASTM D-3410 is detailed in Figure 22.

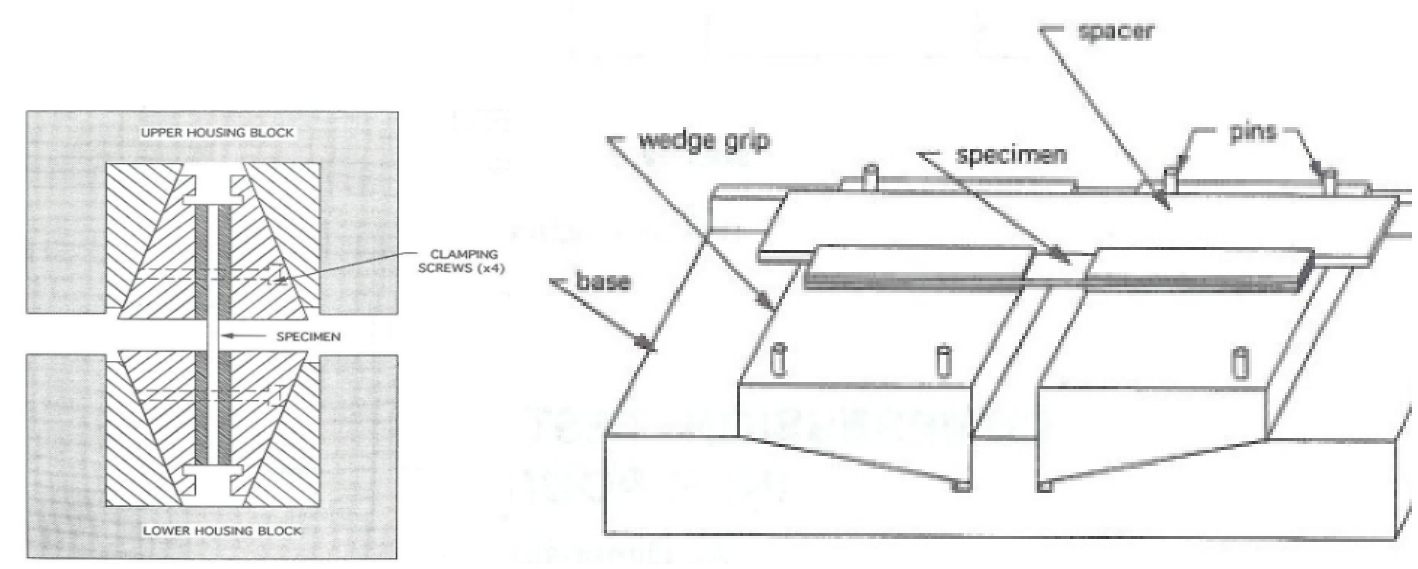

Figure 22: ASTM D-3410 Wedge Grip Example

The actual testing wedge grips are Instron 2743 Wedge Grips with 100 kilo Newton load cell. The Instron wedge grips are quite similar to the example given in Figure 1.6 but the gripping dimensions are a bit smaller. Due to the limited gripping dimensions, the actual test specimen geometry is a bit shorter in length. Strain gages are also placed on several of the test specimens in order to determine the strain in the horizontal direction. This strain is used in correlation with the vertical strain to determine Poisson's ratio of the fiberglass specimen. 
The test rates and test environment are the same as in ASTM D-3039. The only difference is the direction the load is applied and the failure modes and areas. Some of the acceptable failure modes and areas include angled, brooming, transverse shearing, and throughthickness conditions at the edge of the grip and gage area. A detail example of the failure modes and areas is displayed in Figure 23 [4].
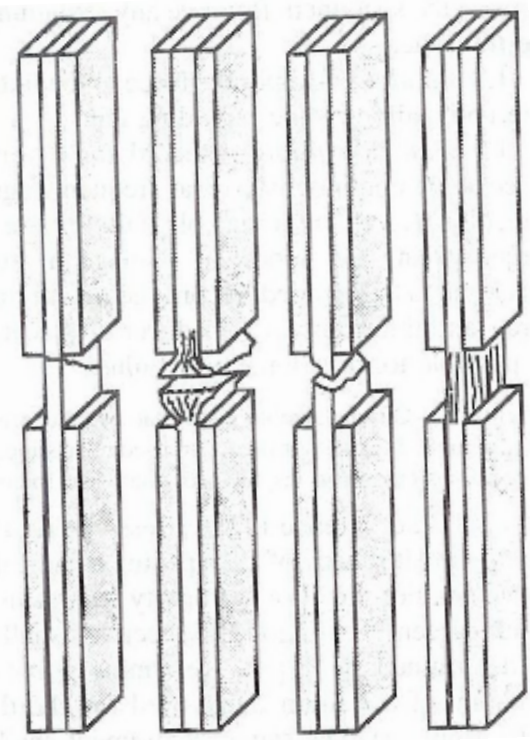

Figure 23: Acceptable Failure Modes and Areas

\subsubsection{ASTM D-3171 Standard Test Methods for Constituent Content of Composite}

\section{Materials}

The ASTM D-3171 standard was used to determine the constituent content of the composite sandwich skin. The idea of this particular standard provides a uniform method for collecting the material weight and volume percent differences between the matrix and reinforcement matrix. In this case, the matrix material would be the West Systems 105/206 epoxy and the reinforcement material would be the 18 oz fiberglass woven roving from Jamestown Distributors. This composite skin was removed by a prefabricated composite sandwich structure in order to provide the most accurate result due to different epoxy saturations between the fiberglass skin and the foam core. 
The ASTM D-3171 standard provides two different approaches to measure and calculate for the weight and volume fraction of the composite material. The procedure starts off with determining the easily found known variables. These variables include the density of each individual material components; which is usually found in the manufacturer's specification data, measured area and volume of the test specimen, and lastly the initial weight of the specimen.

The standard starts off with some design requirements for the test specimen and first test method, which includes some criteria to provide the best results [5]:

- $\quad$ Sampling of Test Specimens

- $\quad$ Test Specimen Geometry

- $\quad$ Specimen Cutting

- $\quad$ Specific Conditioning

With sampling of test specimens, the ASTM D-3171 standard states that the minimum test specimens should be no less than three. The more test specimens, the more accurate the result become by eliminating more of the errors associated with experimental testing. The test specimen geometry should also maintain a mass of no less than 0.5 grams, which equates to approximately $0.011 \mathrm{lbs}$, and should have some volume of the test specimens. The specimen cutting process is one of the most important parts of this standard. The reason for this is that an improper cut can lead to frays, delamination, and even trap contaminates in the test specimens giving the incorrect density. Lastly, the specific conditioning references how to dry the test specimen in order to put it in a state of equilibrium.

The testing process is the easiest step. The measured test specimens are then placed in a furnace at a high temperature to remove the matrix material. Once the entire matrix is removed through the "cooking" process, the leftover reinforcement material is then measured and used to calculate the weight and volume fraction. Additional details regarding the requirement to "cook" 
the test specimens are listed in the ASTM D-3171 standard. The equations for calculations are also provided in the standard.

\subsubsection{ASTM C-364 Standard Test Method for Edgewise Compressive Strength of Sandwich Constructions}

The ASTM C-364 standard provides a test method in order to get the compressive properties of any composite sandwich structure where the direction is parallel to the sandwich facing plane, also known as the edgewise testing configuration. According to the standard, the material core can be pretty much anything that is continuous or discontinuous and the foam core chosen for this thesis project meets the criteria. The standard was followed to determine the compressive material properties of the foam core, wood core, and arrestment key.

The ASTM C-364 standard was not followed in full, but a variation of this standard was used to investigation a different design configuration of the composite sandwich structure under buckling loading. For this thesis project, there were many modifications made from the standard in order to support the necessary conditions. These conditions include buckling loading and limited accessibility of the test equipment. Instead, the modifications were made with a different test jig design and test specimen configuration. The test jig was designed to form a U-channel and provide a pinned condition with a 0.25 inch diameter hole. The test specimen was laid up with foam and wood cores. Two wood cores are laid at each edge of the foam core to increase loading capacity and holes are drilled through the wood cores to create a pinned-pinned case for the optimum buckling condition. The results detailing the geometric buckling show that after 9 inches anvil length there is no compression; only buckling occurs with a cross-sectional dimension of 0.75 inch by 0.575 inch. The 11 inch foam length was chosen for convenience of machining. 
Even with all the modifications, a lot of ASTM C-364 standard were followed. This includes the following [6]:

- System Alignment

- Test Environment

- Sampling Size of Test Specimen

- $\quad$ Speed of Testing

System Alignment - This is quite an important aspect of the testing. As stated in the standard, unintended loading eccentricities will cause premature failure [6]. The usual causes for this are poor alignment of the test fixtures, test specimens, or the grips. This is one reason why a modification was made to the jig to eliminate the grip alignment issue and directly mount it to the displacement machine.

Test Environment - The environmental condition can affect the experimental results. Some factors include humidity, temperature, radiation, and cyclic fatigue. The main concern, stated in the standard, is the fluid exposure level. In order to avoid large changes and due to limited resources, the test specimens are kept in the lab room insulated by brick walls.

Sampling Size of Test Specimen - The ASTM C-364 standard states that at least five test specimens are to be tested unless valid results can be gained from fewer specimens [6]. In order to achieve statistically significant results, more test specimens may be needed.

Speed of Testing - Lastly, the speed of the testing section starts with defining the ultimate strength of the structure and can be found from initial trial testing. The suggested standard head displacement rate is 0.020 inch per minute.

\subsection{Specimen Design}

Many of the different designs used for experimental testing have gone through several iterations to eliminate issues and to meet the required conditions. This section of the report will discuss the process that led to the final design, all the limitations and conditions throughout the 
different iterations, and testing criteria that has helped the test specimen design evolve. Such examples are the limitations in dimension set forth by Instron displacement machine to manufacturing imperfections.

\subsubsection{Component Designs of Composite Sandwich Structure}

In order to obtain the mechanic characteristics of several of the components that go into each of the composite sandwich structure, each of these specimen designs are specific in order to obtain the correct experimental results. The manufacturing specifications on several of these components cannot be obtained from the manufacturer or their distributors.

Therefore, experimental testing of these components was required in order to get enough information to formulate a numerical analysis of these composite sandwich structures under buckling loading. The components investigated are the fiberglass skin with cured resin infused, which is also denoted as e-glass composite, and the wood core.

\subsubsection{Wood Core Component Design for Compressive Properties}

The wood core design was used to determine the elastic modulus in two different directions where one direction has the grain parallel to the load vector and the other direction is perpendicular to the same vector. From how the oak wood arrived in the lab, the raw material would be cut down into small blocks that were used during testing. The design of the specimen has the following dimensions where the length of the specimen is parallel to the grain:

Table 6: Wood Core Design Dimensions for Compressive Properties

\begin{tabular}{|c|c|c|}
\hline Width (in) & Overall Length (in) & Thickness (in) \\
\hline 1.0 & 1.5 & 0.5 \\
\hline
\end{tabular}

These designed test specimens would later be placed on the tee shaped jig and compressed to get the load and vertical extension in order to determine the compressive elastic modulus with the given geometry. 


\subsubsection{E-Glass Composite Skin Component Design for Material Properties}

For the data to be standardized, the test specimen's designs followed the recommended dimension and criteria found in the ASTM D-3039 and ASTM D-3410 standard methods to find the tensile and compressive properties of the e-glass composite skin. Each of the standard methods has a different recommended design in order to supply sufficient materials for testing.

Let's start with the design for tensile properties; this was completed in conjunction with another experiment used to determine the full cured trend of the e-glass composite. This experiment will later be discussed. In order to test for tensile properties, the recommended design parameters with slight modification from the ASTM D-3039 standard were used. To refresh, the recommended geometry is listed again in Table 7:

Table 7: ASTM D-3039 Recommended Geometry [3]

\begin{tabular}{|c|c|c|c|c|c|}
\hline $\begin{array}{c}\text { Fiber } \\
\text { Orientation }\end{array}$ & $\begin{array}{c}\text { Width } \\
\text { (in) }\end{array}$ & $\begin{array}{c}\text { Overall } \\
\text { Length (in) }\end{array}$ & $\begin{array}{c}\text { Thickness } \\
\text { (in) }\end{array}$ & $\begin{array}{c}\text { Tab Length } \\
\text { (in) }\end{array}$ & $\begin{array}{c}\text { Tab } \\
\text { Thickness } \\
\text { (in) }\end{array}$ \\
\hline $\begin{array}{c}\text { 0 deg } \\
\text { unidirectional }\end{array}$ & 0.5 & 10.0 & 0.040 & 2.25 & 0.062 \\
\hline $\begin{array}{c}90 \mathrm{deg} \\
\text { unidirectional }\end{array}$ & 1.0 & 7.0 & 0.080 & 1.0 & 0.062 \\
\hline $\begin{array}{c}\text { Balanced and } \\
\text { symmetric }\end{array}$ & 1.0 & 10.0 & 0.100 & Emery cloth & - \\
\hline $\begin{array}{c}\text { Random- } \\
\text { discontinuous }\end{array}$ & 1.0 & 10.0 & 0.100 & Emery cloth & - \\
\hline
\end{tabular}

The actual geometry for the specimen design and configuration are a little different from these recommended design parameters in order to support the limited area that the wedge grip on the Instron displacement machine can grip and to provide comparable results with the actual composite sandwich structure. These parameters are listed in

Table 8:

Table 8: Actual Geometry of Specimen for Tensile Properties

\begin{tabular}{|c|c|c|c|c|c|}
\hline Type & $\begin{array}{c}\text { Width } \\
\text { (in) }\end{array}$ & $\begin{array}{c}\text { Overall } \\
\text { Length (in) }\end{array}$ & $\begin{array}{c}\text { Thickness } \\
\text { (in) }\end{array}$ & $\begin{array}{c}\text { Tab Length } \\
\text { (in) }\end{array}$ & $\begin{array}{c}\text { Tab } \\
\text { Thickness } \\
\text { (in) }\end{array}$ \\
\hline $\begin{array}{l}\text { 2 Layer } \\
\text { E-Glass }\end{array}$ & 1.0 & 7.0 & 0.045 & 1.0 & 0.062 \\
\hline
\end{tabular}


With these dimensions, a total of 96 specimens were manufactured and tested. Also, another difference between the actual specimen design and the one in the standard is that there is no taper section on the tabs or any on the tabs placed at the ends of the test specimens. The ASTM D-3039 standard also states that taper is just a recommendation and is not necessarily needed. An example of these test specimens with and without the tapered tabs is shown in Figure 24. The top image has no taper tabs and the bottom diagram shows taper tabs.

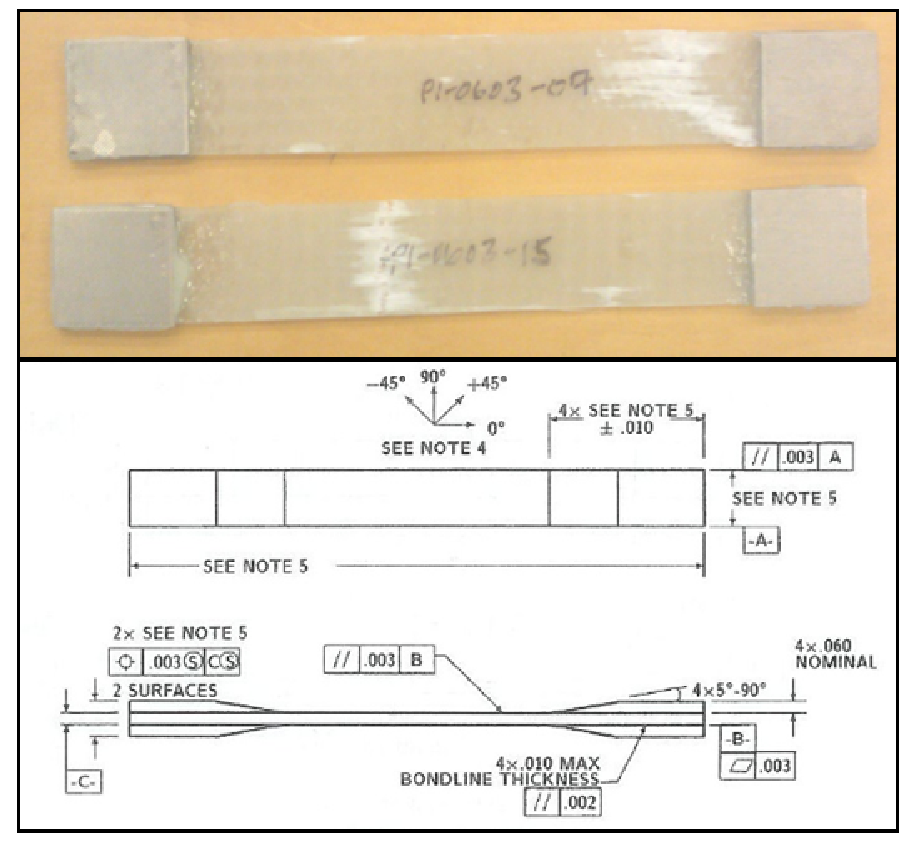

Figure 24: Tapered versus No Tapered Tabs

In order to get the compressive properties of the e-glass skin, the design of the specimen follows ASTM D-3410 standard method with a slight deviation. This deviation, as discussed earlier, is due to the limitation set forth by the Instron displacement machine. The recommended test geometry stated in the ASTM standard is listed again in 
Table 9. 
Table 9: ASTM D-3410 Recommended Geometry [4]

\begin{tabular}{|c|c|c|c|c|c|}
\hline $\begin{array}{c}\text { Fiber } \\
\text { Orientation }\end{array}$ & $\begin{array}{c}\text { Width } \\
\text { (in) }\end{array}$ & $\begin{array}{c}\text { Gage Length } \\
\text { (in) }\end{array}$ & $\begin{array}{c}\text { Tab Length } \\
\text { (in) }\end{array}$ & $\begin{array}{c}\text { Overall Length } \\
\text { (in) }\end{array}$ & $\begin{array}{c}\text { Tab } \\
\text { Thickness } \\
\text { (in) }\end{array}$ \\
\hline $\begin{array}{c}\text { 0 degrees } \\
\text { unidirectional }\end{array}$ & 0.5 & $0.5-1.0$ & 2.5 & $5.5-6.0$ & 0.06 \\
\hline $\begin{array}{c}90 \text { degrees } \\
\text { unidirectional }\end{array}$ & 1.0 & $0.5-1.0$ & 2.5 & $5.5-6.0$ & 0.06 \\
\hline $\begin{array}{c}\text { Specially } \\
\text { Orthotropic }\end{array}$ & 1.0 & $0.5-1.0$ & 2.5 & $5.5-6.0$ & 0.06 \\
\hline
\end{tabular}

The dimensions used to create slightly deviates from the above table and are listed in

Table 10:

Table 10: Actual Geometry of Specimen for Tensile Properties

\begin{tabular}{|c|c|c|c|c|c|}
\hline Type & $\begin{array}{c}\text { Width } \\
\text { (in) }\end{array}$ & $\begin{array}{c}\text { Gage Length } \\
\text { (in) }\end{array}$ & $\begin{array}{c}\text { Tab Length } \\
\text { (in) }\end{array}$ & $\begin{array}{c}\text { Overall Length } \\
\text { (in) }\end{array}$ & $\begin{array}{c}\text { Tab } \\
\text { Thickness } \\
\text { (in) }\end{array}$ \\
\hline $\begin{array}{c}\text { 2 Layer } \\
\text { E-Glass }\end{array}$ & 1.0 & 1.0 & 2.0 & 5.0 & 0.062 \\
\hline
\end{tabular}

The ASTM D-3410 standard method also provides the recommended dimensions to use for finding the compressive material properties. The dimensions in the ASTM D-3410 standard creates a collective data set of material properties that are better comparably to other data sets, but this recommended geometry would not matter for these test specimens because it provides a small enough anvil geometry in order to retrieve the compressive properties and large enough geometry to provide the necessary support for compression on these test specimens. In addition, strain gages were placed on several test specimens that were used for compression in order to determine the compression Poisson's ratio. 
An example of deviated design is displayed in Figure 25:

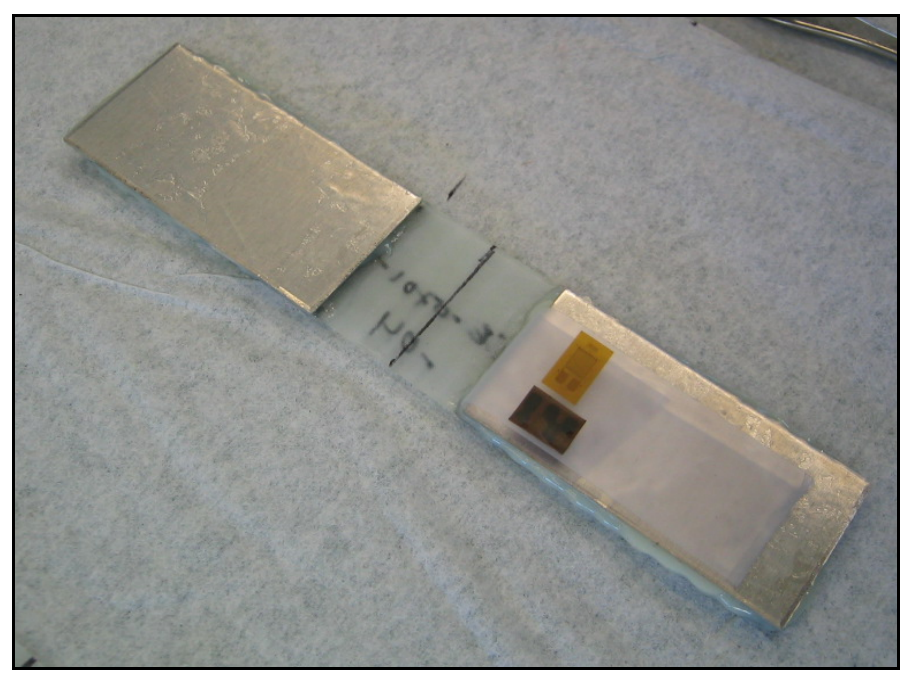

Figure 25: E-Glass Deviated Design for Compressive Properties

\subsubsection{E-Glass Composite Design for Weight and Volume Fractions}

There were a couple of requirements that drove the design of these specimens which were used to obtained the weight and volume fractions. The first limitation was the heating chamber of the muffle furnace, which has a width 4 inches, height of 3.75 inches, and depth of 4.25 inches. Additional information regarding the furnace can be found in the Appendix.

The second limitation was the removal of the skin from the composite sandwich structure in order to provide the most accurate fiber-matrix content. This further limited the size of the test specimens because the composite sandwich structure dimensions were derived from the test jig dimensions.

Therefore, these driving factors allowed for the following dimensions:

Table 11: E-Glass Composite Design for Fraction Analysis

\begin{tabular}{|c|c|c|}
\hline Width (in) & Length (in) & Thickness (in) \\
\hline 0.750 & 1.000 & 0.045 \\
\hline
\end{tabular}

In order to get the test specimen to the correct dimension and conditions, it had to be removed from an existing composite sandwich structure and chopped down to the appropriate dimensions. Examples of this test specimen before and after are displayed below: 


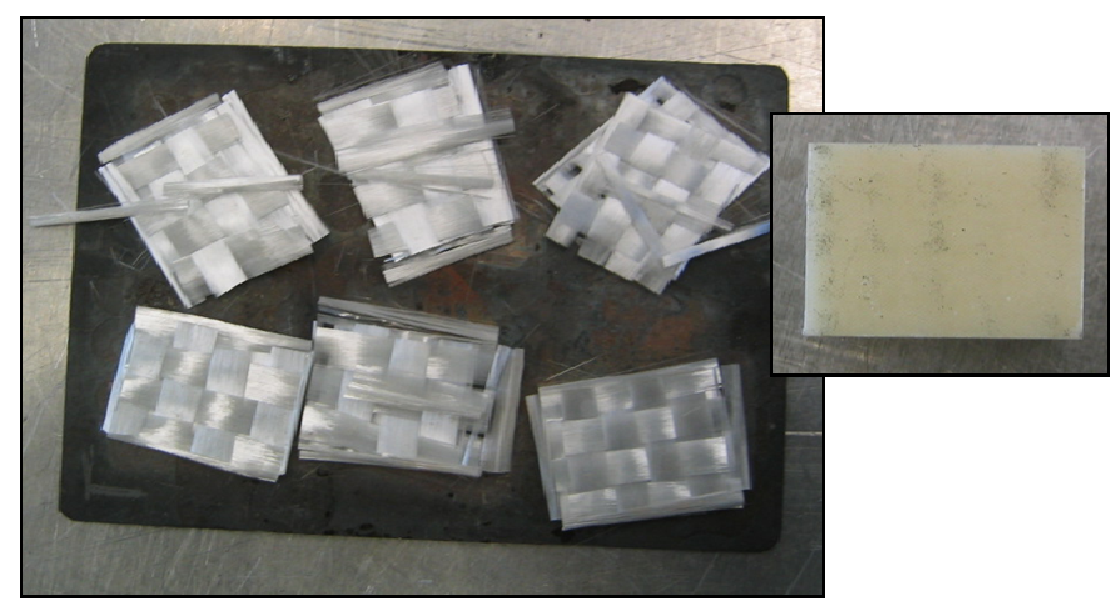

Figure 26: Examples of E-Glass Design for Fraction Analysis

\subsubsection{Delamination Arrestment Key Design}

In order to design what a delamination arrestment key will look like, the function of the key must be understood. The idea is to introduce a stiffener into the composite sandwich structure in order to transfer loads appropriately across the whole composite sandwich structure even when there is a "break" in the structure, such as a delaminated region, and reduces the need for repairs.

This led the arrestment key design to have a semi-circular cross-sectional area and varies in length based on the composite sandwich structure configuration. The semi-circular crosssectional area is designed to embed easily into the grooves of the foam core and bond to its contact surfaces. The shape is also designed to maintain its structural integrity with its curved surfaces. For this project, the semi-circular shape was used, but future works could be done in order to determine the optimal geometry.

Like many composite structures, the weight does matter. These delamination arrestment key are made of fiberglass strand hand cured with epoxy and pressurized to obtain its shape. Note that the content of fiber to matrix is different between the skin and the key due to the different manufacturing process. 
The geometry of the delamination arrestment key is detailed in the table below:

Table 12: Delamination Arrestment Key Geometry

\begin{tabular}{|c|c|}
\hline Type & Value \\
\hline Diameter (in) & 0.250 \\
\hline Radius (in) & 0.125 \\
\hline Length (in) & $0.750-14.000$ \\
\hline
\end{tabular}

Examples of the delamination arrestment key are displayed in Figure 27. The left image shows the key after it has been cut down, but still required sanding to get it into tolerance. The right image details the various lengths the key can be and that it is dependent on the configuration.

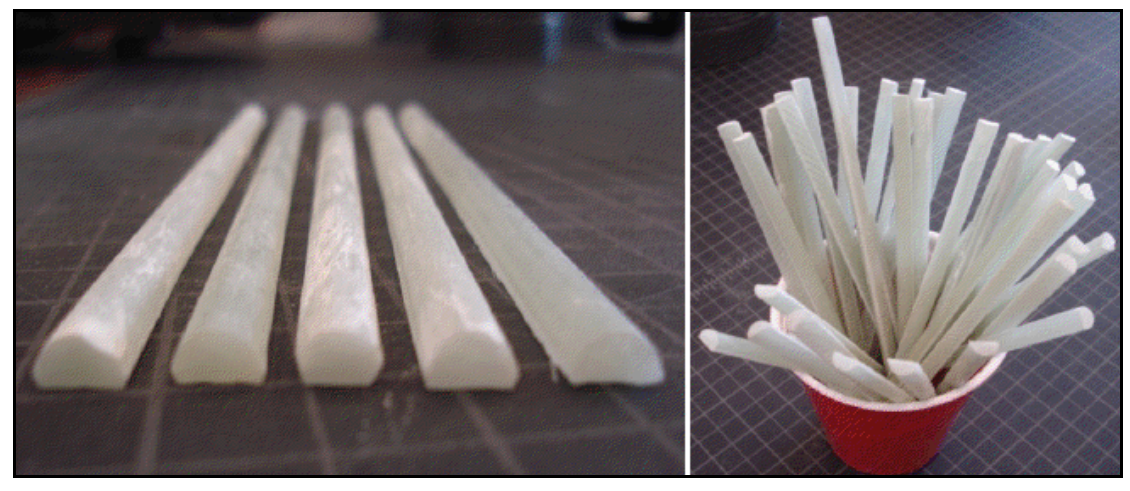

Figure 27: Delamination Arrestment Key Examples

\subsection{Delamination Arrestment Key Design for Mechanical Properties}

In order to prepare the further testing of the arrestment key to get its mechanical characteristics, the design had to meet some criteria. One of these criteria is to have a specimen that does not buckle if loaded. The other criteria are to have a sufficient amount of material to accuracy determine the content within the keys. In so, the cross-sectional area of these keys did not change but the length was set to 1 inch. This length did not buckle the key when loaded and had enough material for a fiber-matrix content test. 


\subsection{Delamination Arrestment Key Mold Design for Manufacturing}

The delamination arrestment key mold design is derived from how many keys can be made per layup. To optimize the amount of output, an aluminum plate that is 12 inches by 12 inches with 0.5 -inch thickness was milled out to create the necessary grooves to shape the keys. The design of the key mold is meant to be simple so that it allows for manageability from laying up the fiberglass strands to removing it from the plate. An example of the mold design is detailed in Figure 28.

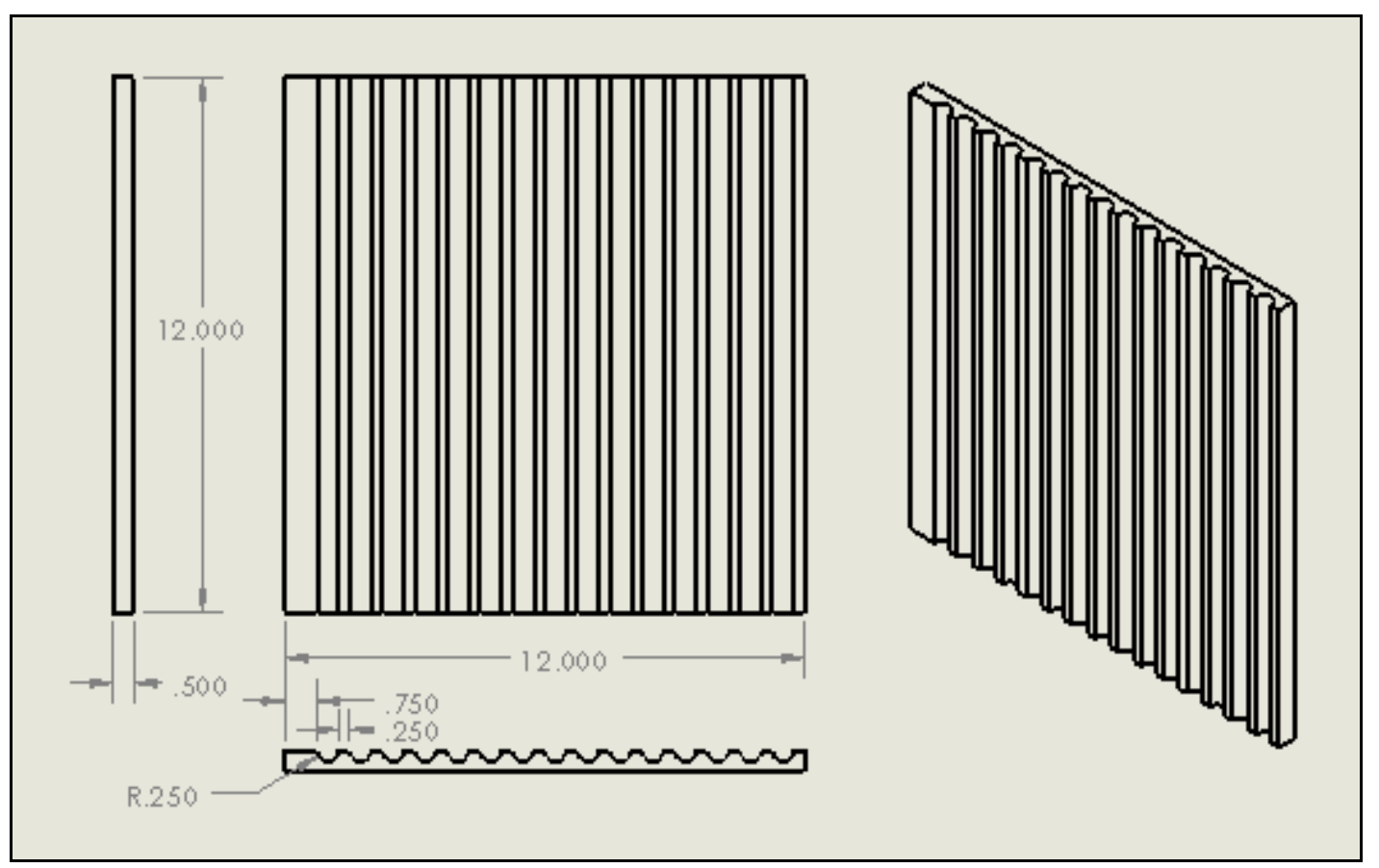

Figure 28: Delamination Arrestment Key Mold Design

\subsection{Specimen Design for Buckling Length}

The study regarding the buckling length is referring to what length does a specimen have to be in order for it to exhibit pure buckling and no compression. From this, it can be concluded that the cross-sectional area of the composite sandwich structure is the same throughout all the test specimens and the only thing that varies is the length per an inch increment.

The specimen design consists of the two layer e-glass skin, wood boundary cores, and a foam core all bonded together using a vacuum resin infusion process. The previous design used 
did not contain the wood boundary cores and was unsuccessful in determining if the specimen would buckle per given length. The reason is the lack of manufacturing accuracy. The cut edge would be uneven and due to how thin the skins are the failure would happen at the boundary before buckling could even occur. The second iteration of this specimen was the introduction of the wood boundary cores where the idea is to remove the issue caused by the skin and make it pinned through the composite skin and the wood base. An example of what it looks like after it has been tested can be seen in Figure 29. Note the damages the around the holes.

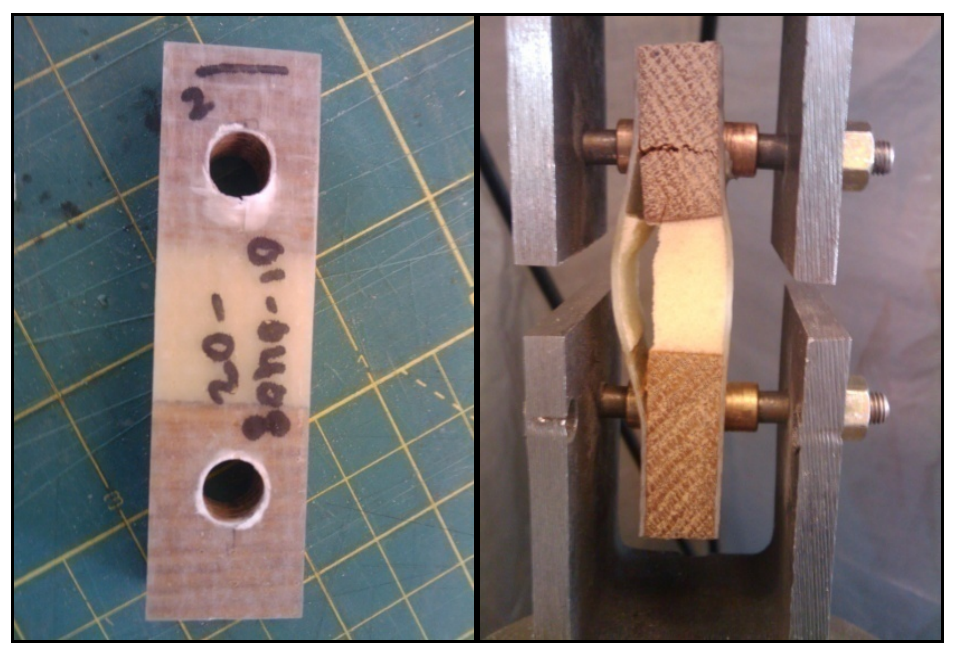

Figure 29: Second Generation Specimen and Wood Grain Issue

So with the damage issues, a third iteration of this specimen was made where the holes are 90 degrees and only through the wood boundary cores. This would allow the test specimen to have a true pinned-pinned boundary and remove the hole damage caused by the moment of inertia. This change did help the structure but another issue arises when the direction of the wood boundary core matters. The wood grain placed perpendicular to the load vector could not maintain the loading capacity, thus a rotation of the wood base would have the grain parallel to the load vector and solve the load capacity issue. The issue can be seen in Figure 29 and the grain parallel example can be seen in Figure 30. 


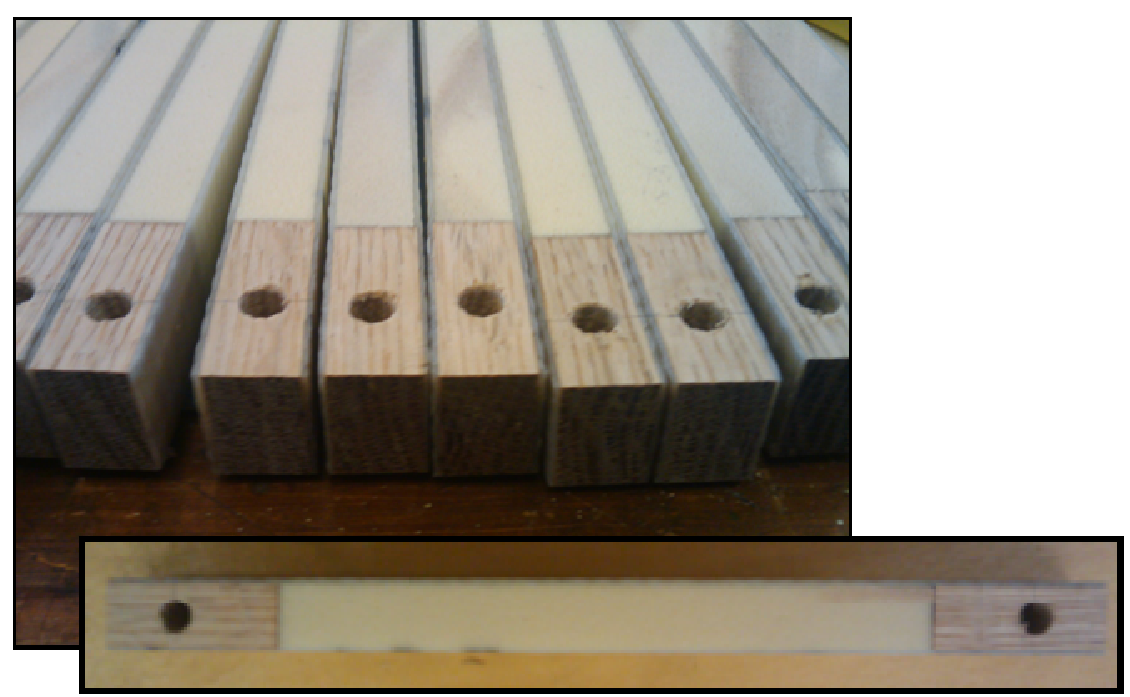

Figure 30: Wood Grain Parallel to Load Vector

The dimensions for the latest design of the buckling length are listed in the Table 13.

Each case study has a different length that varies in height per inch increment.

Table 13: Buckling Length Specimen Dimensions

\begin{tabular}{|c|c|c|c|}
\hline Width (in) & Thickness (in) & Anvil Height (in) & Overall Height (in) \\
\hline 0.750 & 0.585 & $1.000-11.000$ & $4.000-14.000$ \\
\hline
\end{tabular}

\subsection{Specimen Design for Different Configurations}

There are too many different configurations to talk about individual, so this section divides all the attributes of the different configurations and see how these attributes fit together to form each of the different configurations. The subsections of the different attributes include the initial delamination, the grooves within the foam, and the embedment of the arrestment key.

\subsubsection{Initial Delamination}

An initial delamination refers to a way of creating a separation between the composite and the material core usually made of foam or honeycomb. There are many different shapes and ways of introducing this delamination into a composite sandwich structure, but the easiest is to 
add a thin sheet of non-porous, non-stick material into the manufacturing process before the epoxy is fed through the structure.

In this particular case, the initial delamination is a rectangular strip that spans the entire width of the test specimen and varies only in length per each case. There are four different sizes of delaminations that were introduced into various specimen configurations and their sizes range from 0.5 -inch to 2.0 -inch per half an inch increment.

The insertion of these rectangular strips in the appropriate position for the configuration, there is an insignificant amount residual stress from the non-porous being in the structure. The strips do not add any strength to the structure at all. Its sole purpose is to only create the initial delamination for all the different configurations.

\subsubsection{Delamination Arrestment Key Embedment and Foam Core Grooves}

There were various ideas on how to integrate these keys into the structures such as bonding them to the exposed skin and even creating discontinuous sections in the structure for the keys to fit properly. The best design is to create grooves in the foam core where the arrestment keys will sit during the manufacturing process. The keys would then be flushed with the contour of the exposed skin and have bonding surfaces throughout.

There are five different layouts used to support all the different configurations. Some of these configurations differ slightly in order to support the initially delaminated regions. Several examples of the plates used for the various configurations starting with the horizontal configuration are shown in Figure 31. 


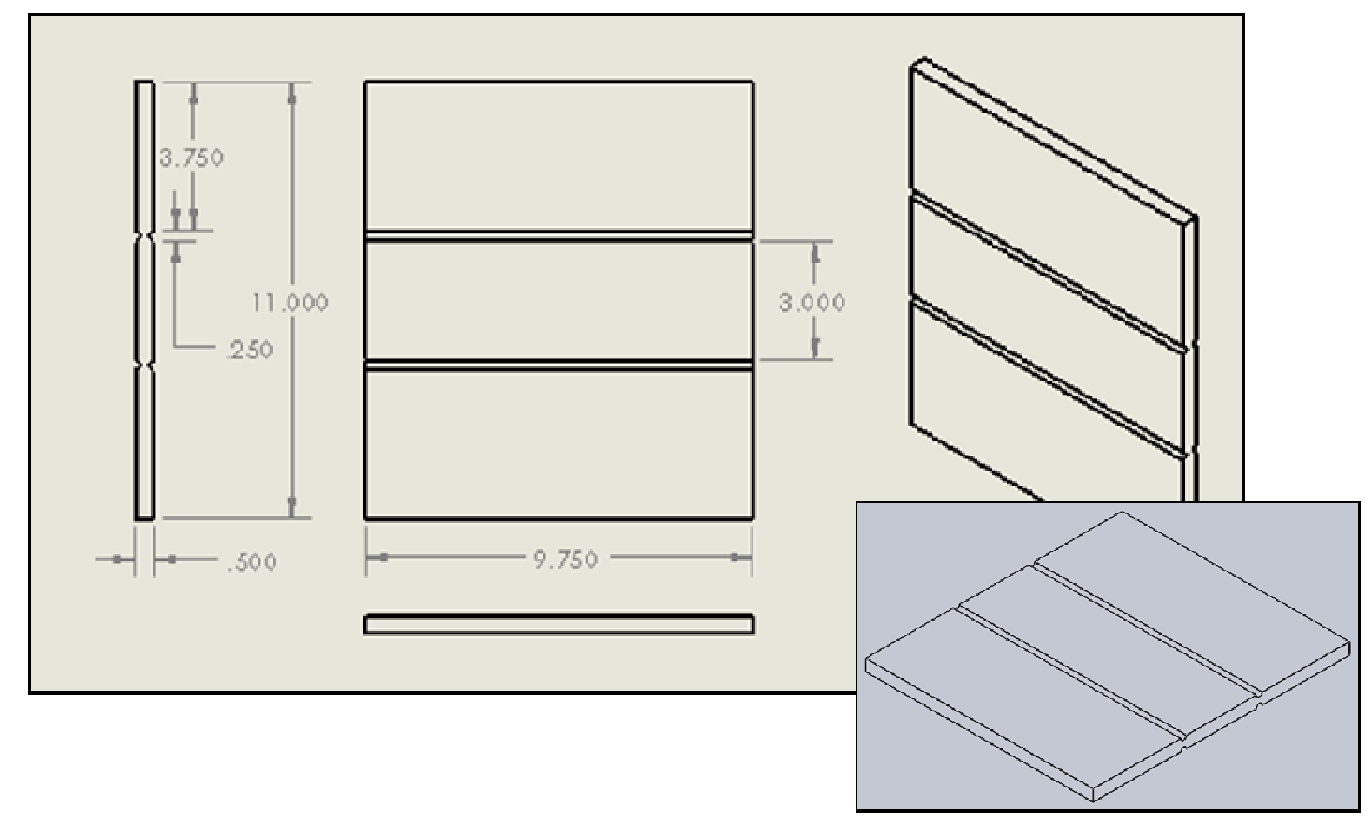

Figure 31: Horizontal Groove Configuration in Foam Core

There are actually two different designs for the horizontal grove configurations where one is to support a 1-inch delamination and the other to support a 2-inch delamination. These design gaps between the delamination regions are made to be constant in structure and comparable in nature. For the above dimensions, this groove configuration is made specifically for a 2-inch delamination providing a 0.5 -inch between each edge of the delaminated region and the arrestment key locations.

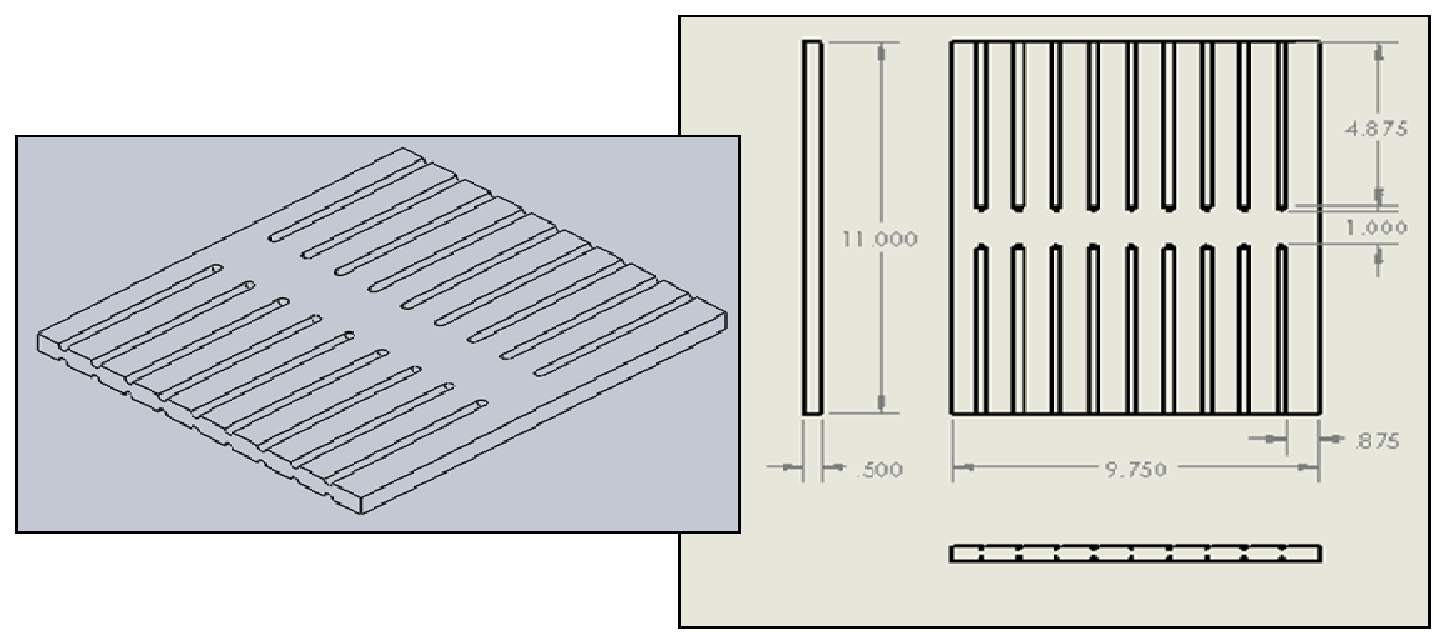

Figure 32: Discontinuous Vertical Groove Configuration in Foam Core 
In Figure 32, the discontinuous vertical groove configuration is limited in how the groove configuration can be manufactured, hence adding additional variability into the composite sandwich structure. That variability in the structure cannot be prevented. It is impossible to mill the correct flat ends for the grooves due to the nature of rotating milling bit. The milling bit will always make rounded ends for the groves. In order to reduce this manufacturing limitation, during manufacturing these small gaps are filled with epoxy and processed with the keys and the rest of the components.

This particular groove design is used for the discontinuous key configuration with either a 1-inch or 2-inch delamination introduced into the composite sandwich structure. The gap between the discontinuities varies up to 2 inches depending on the delamination length.

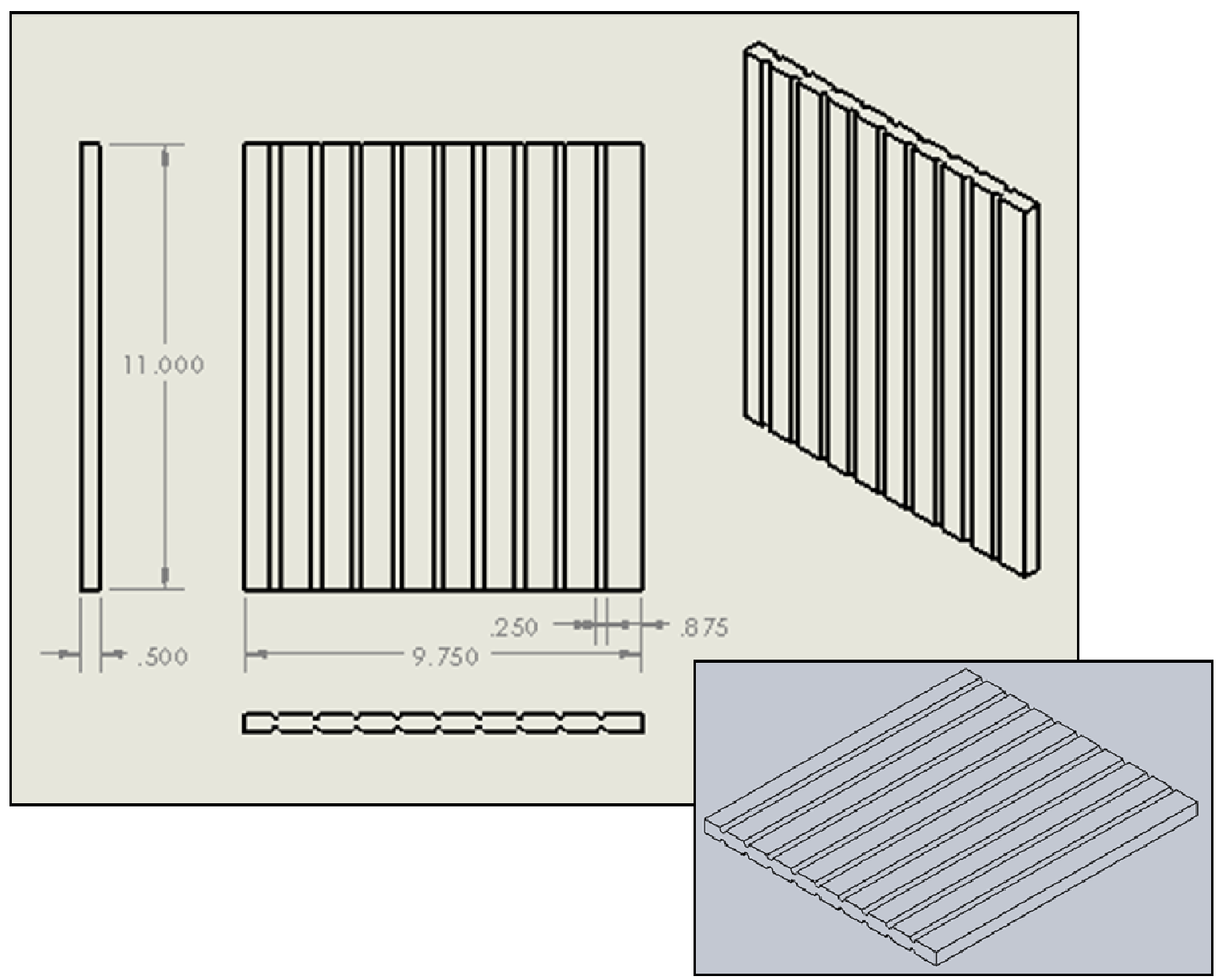

Figure 33: Full Continuous Groove Configuration in Foam Core 
The last groove configuration is a full continuous groove across the entire structure. This can be seen in Figure 33. This particular configuration is used in the manufacturing of the continuous key design with and without the initial delamination. This design was used the most where there was a configuration with the continuous key and no delamination, continuous key with delaminated regions where the skin delaminates between the key and foam, and the continuous key with a delamination where the skin and key delaminate from the foam.

Of all the different attributes, these form all the test specimens used throughout this project. Examples of the specimen are displayed in Figure 34.

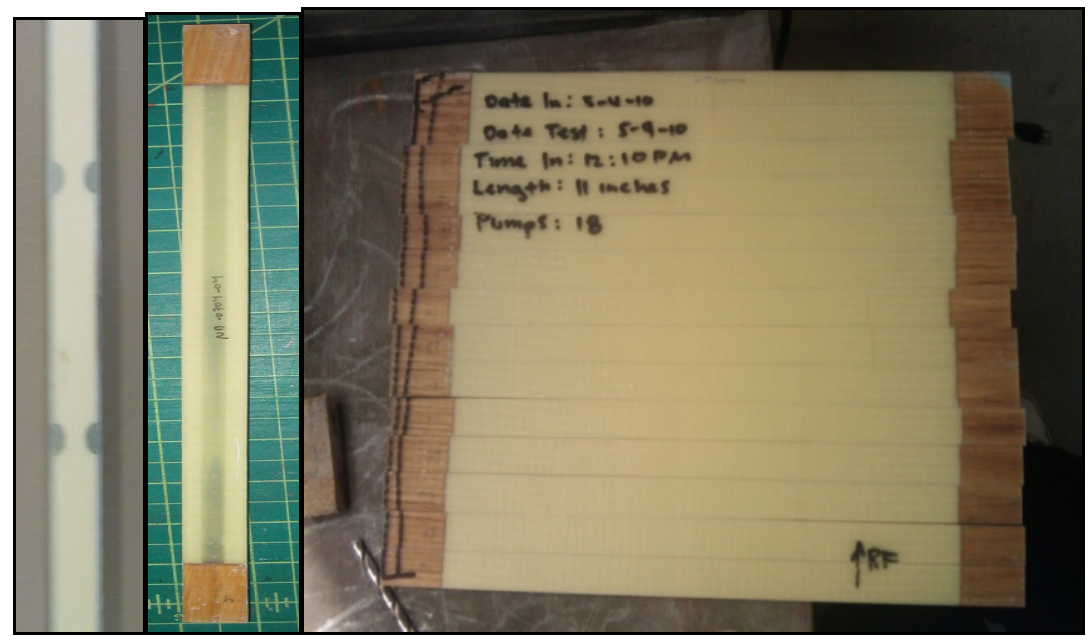

Figure 34: Examples of Different Configurations

\subsection{Material Selection and Mechanical Characteristics/Properties}

This section provides a discussion of the materials used throughout this thesis project in regards to why these materials were chosen. Also, the material properties and characteristics used in this thesis project will be investigated.

The composite sandwich structure used for this thesis project consists of fiberglass woven roving, foam core, oak wood core, and the plastic epoxy. There were also other materials used through the manufacturing process of the composite sandwich structure, but those materials were only used to create the environment for the sandwich structure and discarded after their use. The materials selected for the composite sandwich structure are dependent primary on building upon 
the foundation laid down by the senior project that was discussed in Section 1.4.7.1. The materials are found mainly in the boating industry.

\subsubsection{Foam Core Material Selection and Properties}

The foam core material was chosen to match what is used in the boating and aerospace industries. The foam core used for this project is a LAST-A-FOAM FR-6710 foam material purchased from General Plastics Manufacturing Company. According to General Plastics, the foam is a CFC-free, closed-cell, and a flame-retardant polyurethane material with a density of 10 pounds per cubic foot [28].

This particular foam differs from the one used in the senior project but provides the necessary properties to produce a buckled structure with a smaller geometric volume. This entails less material use and cost less to produce the manufactured parts. Note that there is an excess of 300 composite sandwich specimen made and tested throughout the span of this thesis project.

With the limited resources, the foam material was purchased in bulk between a few thesis students to further reduce the overall cost.

One thing to note about the foam material is that there is a slight difference between material properties parallel and perpendicular to the rise direction. The rise direction is referring to the direction the foam was grown. Between the two different directions, there is less than a $5 \%$ difference according to the manufacturing data [28]. The experimental testing for the foam material properties determined that it was much less than a 5\% difference. Thus, the manufacturing of the composite sandwich structure did not take into consideration foam direction because of the small mechanical difference.

The material properties for the foam from the manufacturer are listed in

Table 14. Note that the strength of the material is referring to the ultimate capacity that the material can resist without any deflection. The modulus is referring to the slope of stress versus strain curve which defines the material. 
Table 14: Foam Core Material Properties and Characteristics

\begin{tabular}{|l|c|c|}
\hline \multicolumn{1}{|c|}{ Type } & Value & Units \\
\hline Manufacturer & General Plastics Manufacturing Co. & - \\
\hline Model & LAST-A-FOAM FR-6710 & - \\
\hline Density & 10 & $\mathrm{lbs}^{\mathrm{f}} \mathrm{ft}^{3}$ \\
\hline Tested Temperature & 75 & degrees Fahrenheit \\
\hline Compressive Strength (Parallel) & 10952 & $\mathrm{psi}$ \\
\hline Compressive Strength (Perpendicular) & 11237 & $\mathrm{psi}$ \\
\hline Shear Strength (Parallel) & 239 & $\mathrm{psi}$ \\
\hline Shear Strength (Perpendicular) & 253 & $\mathrm{psi}$ \\
\hline Tensile Strength (Parallel) & 302 & $\mathrm{psi}$ \\
\hline Tensile Strength (Perpendicular) & 306 & $\mathrm{psi}$ \\
\hline Tensile Modulus (Parallel) & 13037 & $\mathrm{psi}$ \\
\hline Tensile Modulus (Perpendicular) & 12691 & $\mathrm{psi}$ \\
\hline Flexural Strength (Parallel) & 479 & $\mathrm{psi}$ \\
\hline Flexural Strength (Perpendicular) & 406 & $\mathrm{psi}$ \\
\hline
\end{tabular}

\subsubsection{Wood Core Material Selection and Properties}

Due to the needed pinned-pinned boundaries, the test specimens needed to be manufactured with a stronger material at the boundary. This required a stiffer material that is able to bond with the fiberglass skin and has the same dimension as the foam core. The material used for the boundary is oak wood and is used in the composite sandwich structure that supports the boundaries of the structure under in-plane displacement. The oak wood core was also chosen to provide enough stiffness to maintain the integrity of the hole's boundary locations. If the holes were manufactured into the foam core, it would saw through the test specimen.

The oak wood was the best choice for its stiffness and its significantly high loading capacity. One note that was considered during the manufacturing process was the grain direction of the oak wood material; hence the direction of how to manufacture the wood core into composite sandwich structure matters. In the layup of the composite structure, the wood grain direction would be laid up parallel to the load vectors.

The wood core dimensions were easily obtained from the lumber yard and matched the half-inch foam thickness. The cost for this particular hard wood is significantly cheaper than any 
forms of metal for the specific volume. There were other hard woods that could have been chosen, but the oak wood provides sufficient loading capacity and was the cheapest in cost.

The experimental testing for material properties are shown in Table 15. The table details results where the grains are either parallel or perpendicular to the loading vectors.

Table 15: Wood Core Material Properties and Characteristics

\begin{tabular}{|l|c|c|}
\hline \multicolumn{1}{|c|}{ Type } & Value & Units \\
\hline Model & Oak & - \\
\hline Compressive Modulus (Parallel) & 30034 & psi \\
\hline Compressive Modulus (Perpendicular) & 71269 & psi \\
\hline
\end{tabular}

\subsubsection{Fiberglass Composite Material Selection and Properties}

The fiberglass composite material used for this thesis project is an 18-ounce fiberglass woven roving obtained from Jamestown Distributors and manufactured by Hexcel Composites. That is, the material density is 18 ounces per square yard and 0.0191 inch thick. The fiberglass material is commonly used for boating and aerospace purposes. Because of the natural characteristics of fiberglass materials, it is able to withstand many different kinds of impact and is much more durable than carbon fiber composite. The fiberglass woven roving is detailed in Table 16 [30]:

Table 16: Fiberglass Composite Material Properties and Characteristics [30]

\begin{tabular}{|l|c|c|}
\hline \multicolumn{1}{|c|}{ Type } & Value & Units \\
\hline Manufacturer & Hexcel Composites & - \\
\hline Model & Glass & - \\
\hline Style & 7544 & - \\
\hline Weave & 2 End Plain & - \\
\hline Warp Count & 28 & - \\
\hline Fill Count & 14 & - \\
\hline Warp Yarn & ECG 37-1/2 & - \\
\hline Fill Yarn & ECG 37-1/4 & oz/yard ${ }^{2}$ \\
\hline Fabric Weight & 18 & in \\
\hline Fabric Thickness & 0.0191 & lbf/in \\
\hline Warp Breaking Strength & 700 & lbf/in \\
\hline Fill Breaking Strength & 700 & \\
\hline
\end{tabular}




\subsubsection{Epoxy Material Selection and Characteristics}

The epoxy is used to hold the composite materials together and bonded with the foam and wood cores. The epoxy is a West System 105/206 resin and hardener mix that has a weight mixture ratio of 5 resin to 1 hardener and a cured resin specific gravity of 1.18 [29]. This epoxy is the same adhesive used in the senior project and is commonly used in the boating industry. It also bonds well with the fiberglass skin. The material characteristics from the manufacturer for the West System 105/206 epoxy are detailed in Table 17 [29].

Table 17: Epoxy Material Properties and Characteristics [29]

\begin{tabular}{|c|c|c|}
\hline Type & Value & Units \\
\hline Manufacturer & West System & - \\
\hline Model & $105 / 206$ & - \\
\hline Test Temperature & 72 & ${ }^{\circ} \mathrm{F}$ \\
\hline Mix Ratio by weight & $5.0: 1$ & - \\
\hline Mix Viscosity & 725 & cPs \\
\hline Pot Life of $100 \mathrm{~g}$ & 21.5 & Min \\
\hline $\begin{array}{l}\text { Specific Gravity of Cured } \\
\text { Resin }\end{array}$ & 1.18 & - \\
\hline Hardness@2weeks & 83 & Shore D \\
\hline Compression Yield @ 2 weeks & 11500 & psi \\
\hline Tensile Strength & 7320 & psi \\
\hline Tensile Elongation & 4.5 & $\%$ \\
\hline Tensile Modulus & $4.60 \mathrm{E}+05$ & psi \\
\hline Flexural Strength & 11810 & Psi \\
\hline Flexural Modulus & $4.50 \mathrm{E}+05$ & psi \\
\hline Heat Deflection Temperature & 123 & ${ }^{\circ} \mathrm{F}$ \\
\hline Onset of Tg by DSF & 126 & ${ }^{\circ} \mathrm{F}$ \\
\hline Ultimate $\mathrm{Tg}$ by DSC & 139 & ${ }^{\circ} \mathrm{F}$ \\
\hline Annular Shear Fatigue & 10047 & lbs@100,000 cycles \\
\hline Izod Impact, notched & 0.54 & ft-lbs/in \\
\hline
\end{tabular}

\subsubsection{E-Glass Composite Skin Properties and Characteristics}

In order to understand which composite skin for the sandwich structure is better, a compression study on the fiberglass skin when it is infused with the cured resin is needed. To get the best accuracy of the composite skin, the skin should be removed from the composite sandwich structure and tested to understand its mechanical characteristics. This was not the case for this 
section of the study. Using the same manufacturing process as used for the composite sandwich structure, the fiberglass plate was laid up and cured. The plate was then cut down into strips and tabs were placed on these test specimens.

The skin specimens consist of two layers of the fiberglass roving; this is the same for each side of the cores in order to form the composite sandwich structure. What was found were those two layers of fiberglass woven roving were sufficient in providing the stiffness required to maintain the structural shape. Also, more than two layers per side would increase the cost per test specimen.

The skin was chosen because it is commonly found on the haul of boats. The fiberglass material is also used in other industries including aerospace and automobile for strength, impact resistance, and durability.

The mechanical properties for the fiberglass face sheet with the infused resin can be described as one material because of its linear elastic characteristic. These mechanical properties are detailed in Table 18

Table 18: Fiberglass Skin Mechanical Properties and Characteristics [29]

\begin{tabular}{|l|c|c|}
\hline \multicolumn{1}{|c|}{ Type } & Value & Units \\
\hline Model & E-Glass Skin & - \\
\hline Tensile Modulus (Parallel) & $1.92693 \mathrm{E}+06$ & $\mathrm{psi}$ \\
\hline Compressive Modulus (Parallel) & $2.22128 \mathrm{E}+06$ & $\mathrm{psi}$ \\
\hline
\end{tabular}

\subsubsection{Weight and Volume Fractions of E-Glass Skin}

During the test for the mechanical properties, data regarding the content of the fiberglass skin was also studied. The test required the measurement of the weight of the material before and after it had been placed into a furnace to remove the resin matrix content. In doing so, the following table details the material characteristics of the fiberglass skin. 
Table 19: Fiberglass Skin Weight and Volume Fractions

\begin{tabular}{|c|c|c|c|}
\hline \multicolumn{4}{|c|}{ Fiberglass Skin } \\
\hline \multicolumn{2}{|c|}{ Weight Fraction } & \multicolumn{2}{c|}{ Volume Fraction } \\
\hline Fiber & Matrix & Fiber & Matrix \\
\hline $59.52 \%$ & $40.48 \%$ & $57.97 \%$ & $42.03 \%$ \\
\hline
\end{tabular}

\subsubsection{Delamination Arrestment Key Properties and Characteristics}

In order to have a better understanding of the composite sandwich and its components, the delamination arrestment key was also investigated to determine its mechanical properties. The differences between the fiberglass skin and key characteristics will not always be the same because of the different manufacturing process and has a different fiber to matrix ratio.

Table 20: Epoxy Material Properties and Characteristics [29]

\begin{tabular}{|l|c|c|}
\hline \multicolumn{1}{|c|}{ Type } & Value & Units \\
\hline Model & Arrestment Key & - \\
\hline Compressive Modulus (Parallel) & 187721 & psi \\
\hline
\end{tabular}

\subsubsection{Weight and Volume Fractions of Delamination Arrestment Key}

During the test for the mechanical properties, data regarding the content of the arrestment key was also studied. The test required the measurement of the weight of the material before and after it had been placed into a furnace to remove the resin matrix content. In doing so, the following table details the material characteristics of the fiberglass skin.

Table 21: Delamination Arrestment Key Weight and Volume Fractions

\begin{tabular}{|c|c|c|c|}
\hline \multicolumn{3}{|c|}{ Delamination Arrestment Key } \\
\hline Weight Fraction & \multicolumn{2}{c|}{ Volume Fraction } \\
\hline Fiber & Matrix & Fiber & Matrix \\
\hline $47.46 \%$ & $52.54 \%$ & $45.92 \%$ & $54.08 \%$ \\
\hline
\end{tabular}

\subsection{Buckling Length}

Buckling length for this thesis project is based on geometry where the cross-sectional area is the same through the entire test specimen collection. The length of the specimen varies from 1 inch to 11 inches per 1 inch increment to see which length will produce a perfect buckling 
of the test specimens. A perfect buckling entitles no compression of the composite sandwich structures but only a rotation about the pinned boundaries and buckles the specimen.

For the experimental testing, 10 or more of the same test specimens were tested for each of the discussed lengths. The purpose for testing 10 test specimens per case is to reduce the errors and allow for a more accurate measurement of the case studied. The outliers from the data set for each case were discarded and recalculated to provide a better measurement. Even though having more specimens has a better statistical average, errors can sprout up from other locations such as manufacturing imperfections. The experimental results detailed in Table 22 show the results per each case length. The numbers in Table 22 can be considered as outliers due to an inconsistency in the averages of the ultimate loads and ultimate stresses and falls out of the common trends. These outliers are then removed from the graph and trend line calculations.

Table 22: Experimental Results for Buckling Length

\begin{tabular}{|c|c|c|c|c|c|c|}
\hline $\begin{array}{c}\text { Lengt } \\
\mathbf{h}\end{array}$ & $\begin{array}{c}\text { Slenderness } \\
\text { Ratio }\end{array}$ & $\begin{array}{c}\text { Vertical } \\
\text { Extension }\end{array}$ & $\begin{array}{c}\text { Ultimate } \\
\text { Load }\end{array}$ & $\begin{array}{c}\text { Vertical } \\
\text { Strain }\end{array}$ & $\begin{array}{c}\text { Ultimate } \\
\text { Stress }\end{array}$ & $\begin{array}{c}\text { Horizonta } \\
\mathbf{l} \\
\text { Extension }\end{array}$ \\
\hline in & - & in & lbf & in/in & psi & in \\
\hline 1 & 5.58629 & 0.04358 & 995.42188 & 0.04648 & 2206.56422 & 0.00511 \\
\hline 2 & 11.74121 & 0.05143 & 1305.05053 & 0.02612 & 2910.08920 & 0.00922 \\
\hline 3 & 17.87665 & 0.05963 & 1267.84653 & 0.02009 & 2941.31715 & 0.01274 \\
\hline 4 & 23.67655 & 0.06225 & 1256.44660 & 0.01568 & 2878.66952 & 0.04393 \\
\hline 5 & 29.73425 & 0.06324 & 1098.30648 & 0.01273 & 2506.73170 & 0.03287 \\
\hline 6 & 35.98031 & 0.06477 & 931.48706 & 0.01079 & 2148.59724 & 0.13753 \\
\hline 7 & 41.56688 & 0.07000 & 984.09727 & 0.01014 & 2248.52657 & 0.11821 \\
\hline 8 & 47.06140 & 0.07142 & 842.34922 & 0.00907 & 1883.31489 & 0.15174 \\
\hline 9 & 52.90415 & 0.07562 & 786.83135 & 0.00852 & 1785.63294 & 0.19437 \\
\hline 10 & 58.72629 & 0.09490 & 680.90911 & 0.00961 & 1518.88466 & 0.30626 \\
\hline 11 & 65.43407 & 0.11083 & 685.60279 & 0.01005 & 1545.26154 & 0.42417 \\
\hline
\end{tabular}

The average of the ultimate load for the 1-inch case was removed before it significantly deviates from the trend in Table 22. Noted by the graph, there is a decline in the ultimate loading capacity as the length of the specimen increase. This is a common trend for most materials because the geometry causes the test specimens to deflect horizontally and become unstable. This 
horizontal deflection reduces the vertical strains which correspond to the decrease in vertical inplane loading.

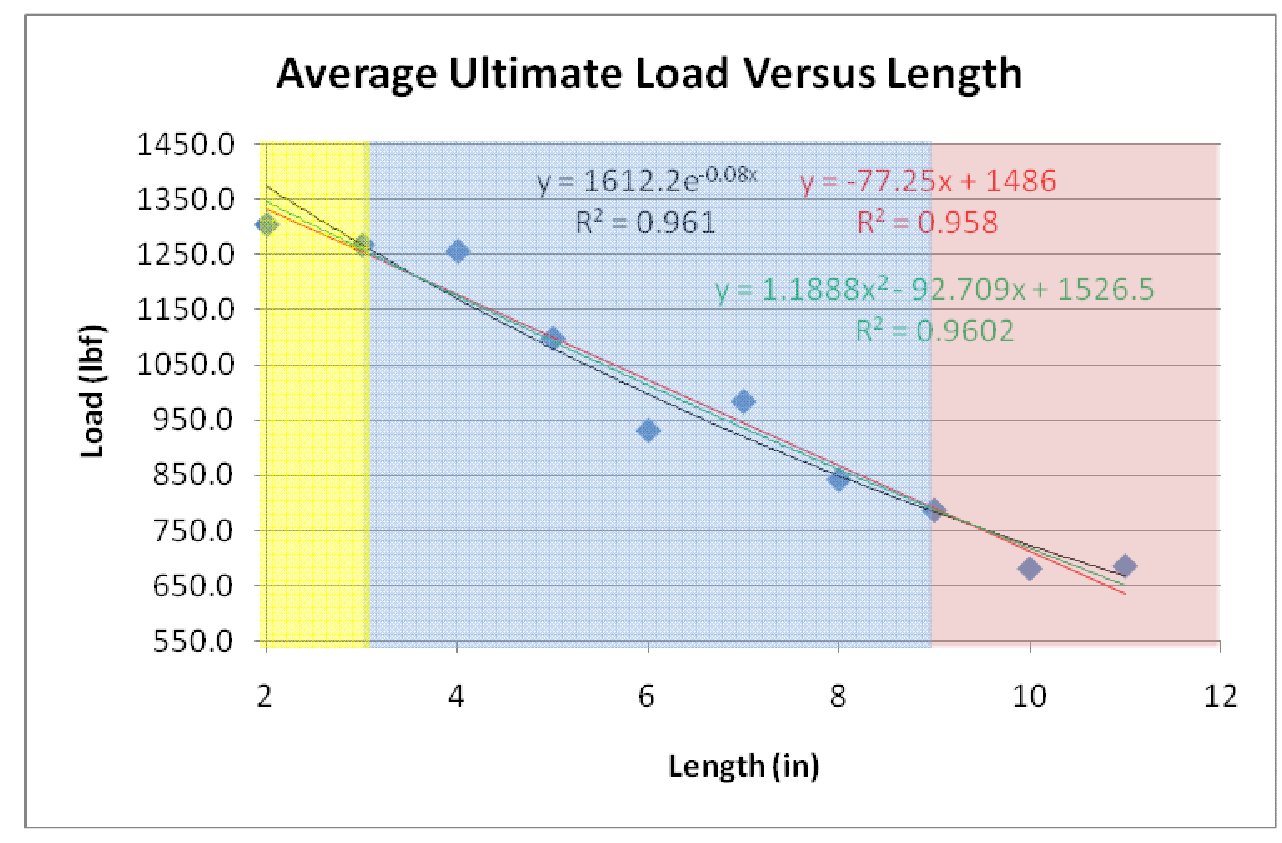

Figure 35: Average Ultimate Load versus Length

The graph is also divided into three different regions where each region designates if it is either pure compression phase, transition phase, or pure buckling phase. The pure compression phase is commonly seen with short specimens where there is no instability and Poisson's ratio is in full effect. The transition phase is a combination of some compression and some buckling in the structure; this is usually seen in immediate length specimens. And lastly, the pure buckling phase is designated for long specimens where the specimen only becomes unstable and sees no compression. The yellow region exhibits the pure compression phase, the blue region is the transition phase, and the purple region is the buckling phase.

Also in Figure 35, three different trend lines are able to represent the data within the range of $96 \%$ coefficient of determination with the 1-inch data removed. The three different trend lines are color coded. The green line and the equation in green represent a parabolic trend. The red trend line represents a linear trend. Lastly, the black trend line represents the exponential trend and best represents the data. Figure 36 shows a similar graph as Figure 35, but it eliminates 
geometry from the graph. The calculated equations can still be represented with geometry. The coefficient of determination for Figure 36 is lower but still accurately represents the decline of stresses as the geometric ratio increases. Similarly, the trend lines are color coded to represent the different trend types. The same colors are used for the same phases in Figure 36.

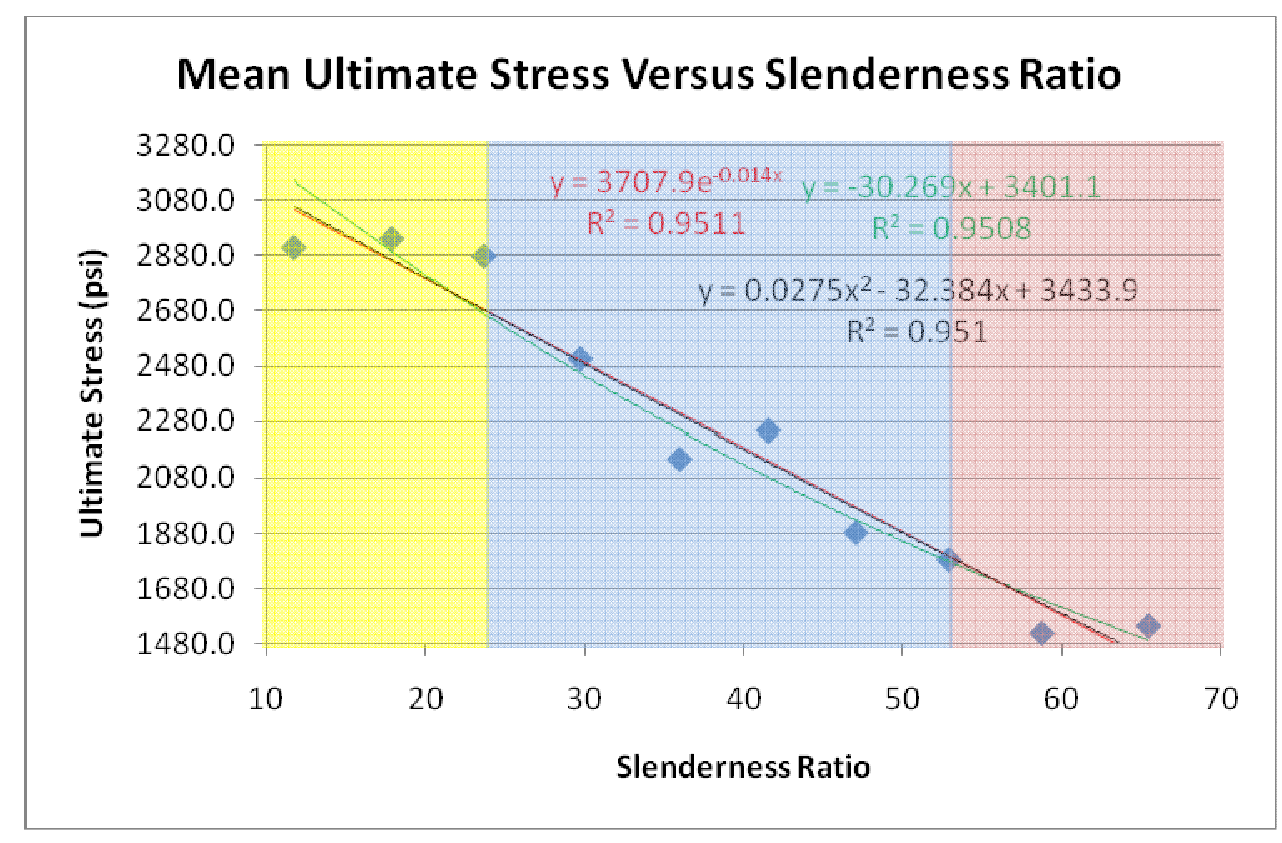

Figure 36: Average Ultimate Stress versus Slenderness Ratio

The horizontal extension is the measure of how much the various lengths of test specimens deflect when subjected to in-plane displacement. The data graph shown in Figure 5.3 shows the average maximum of the horizontal extension per each length case. This graph is strictly based off of measured data. The graph shows an increase in horizontal extension with an increase in the geometric length and same cross-sectional area for all the test specimens. Much of the horizontal extension is due to the test specimen buckling causing the increase in the horizontal deflection. The trend lines detail a good representation with the exponential equation but it is best fitted to a parabolic equation with a $95.9 \%$ coefficient of determination. The parabolic trend line and equation is denoted by the color black. 


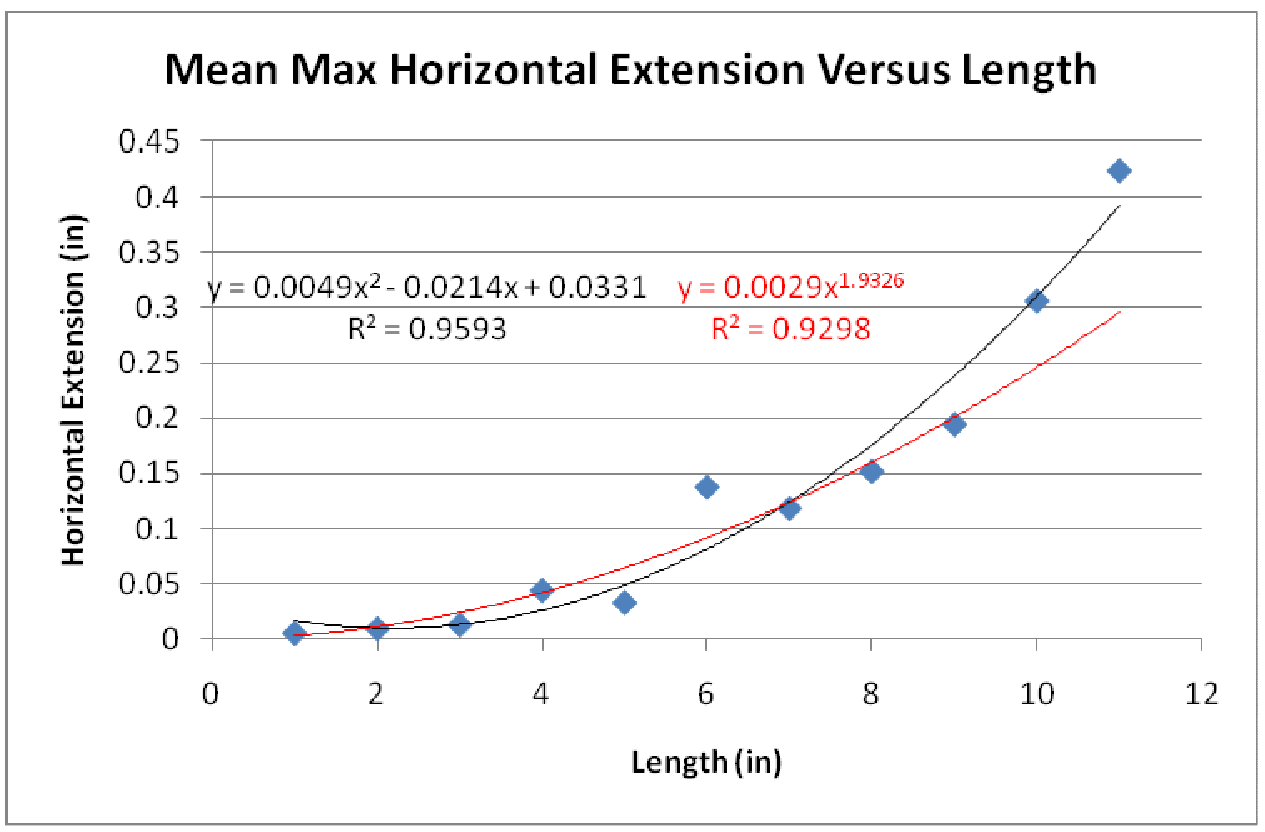

Figure 37: Average Horizontal Extension versus Length

Similarly to Figure 37 , Figure 38 details the vertical extension in relation with the specimen length and shows an increase in extension as the length of the specimen increases. Yet again, a parabolic trend best fits the data and it is represented by the red colored line and equation. For shorter length test specimens, the vertical extension in relation to the horizontal extension is due to Poisson's ratio and it is only subjected to pure compression. For longer length test specimen, the specimen would have more horizontal extension due to the geometry and inplane displacement. 


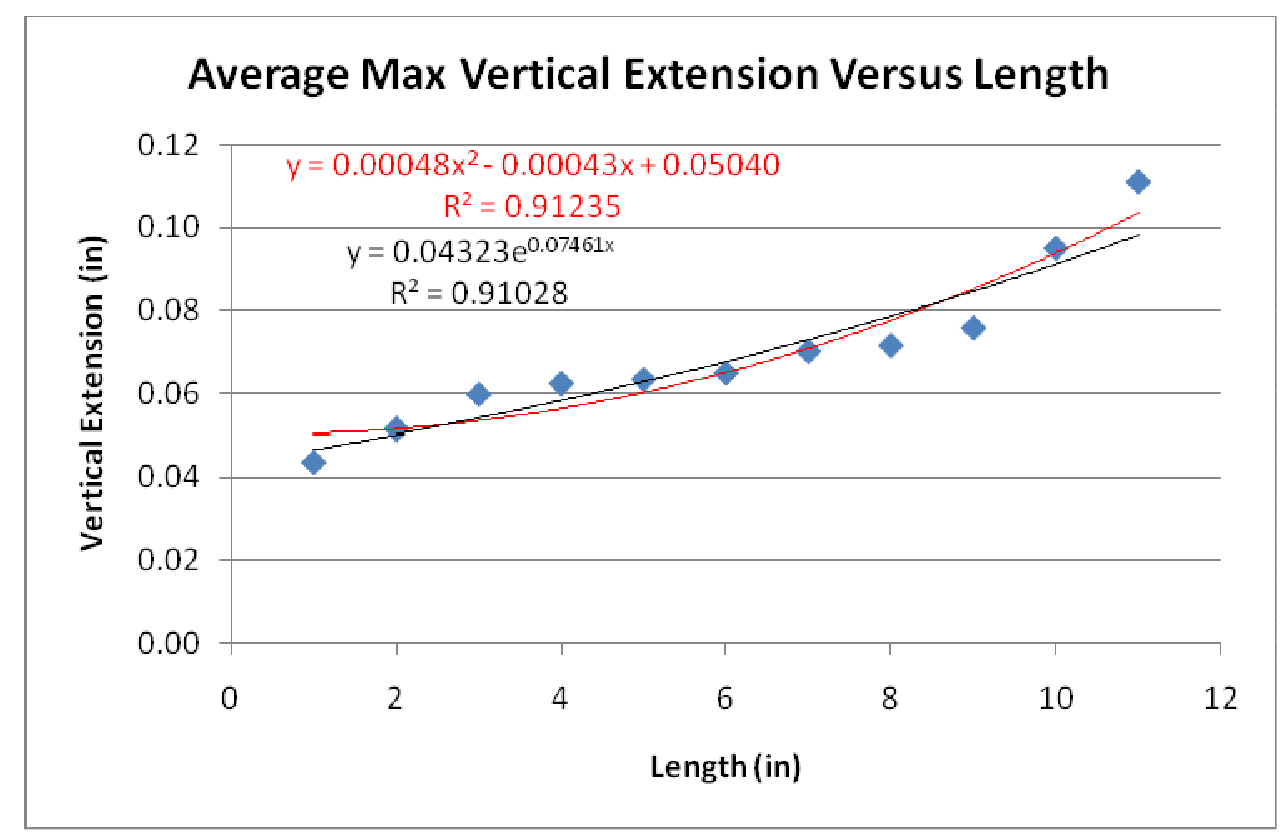

Figure 38: Average Vertical Extension versus Length

The last graph that is used to describe the geometric behavior of the test specimen is

Figure 39, which describes the maximum horizontal extension in relation to the vertical extension for the averages of the specimen cases. This graph shows how much of a change the test specimens experience when the geometry is changed. The longer the specimen is, the more extensions in both directions the specimen sees. The graph can be represented by a linear fit with a coefficient of determination of $93.4 \%$, but it is best represented by a parabolic fit. The linear trend line is coded with the color red and the parabolic is coded with the color black. 


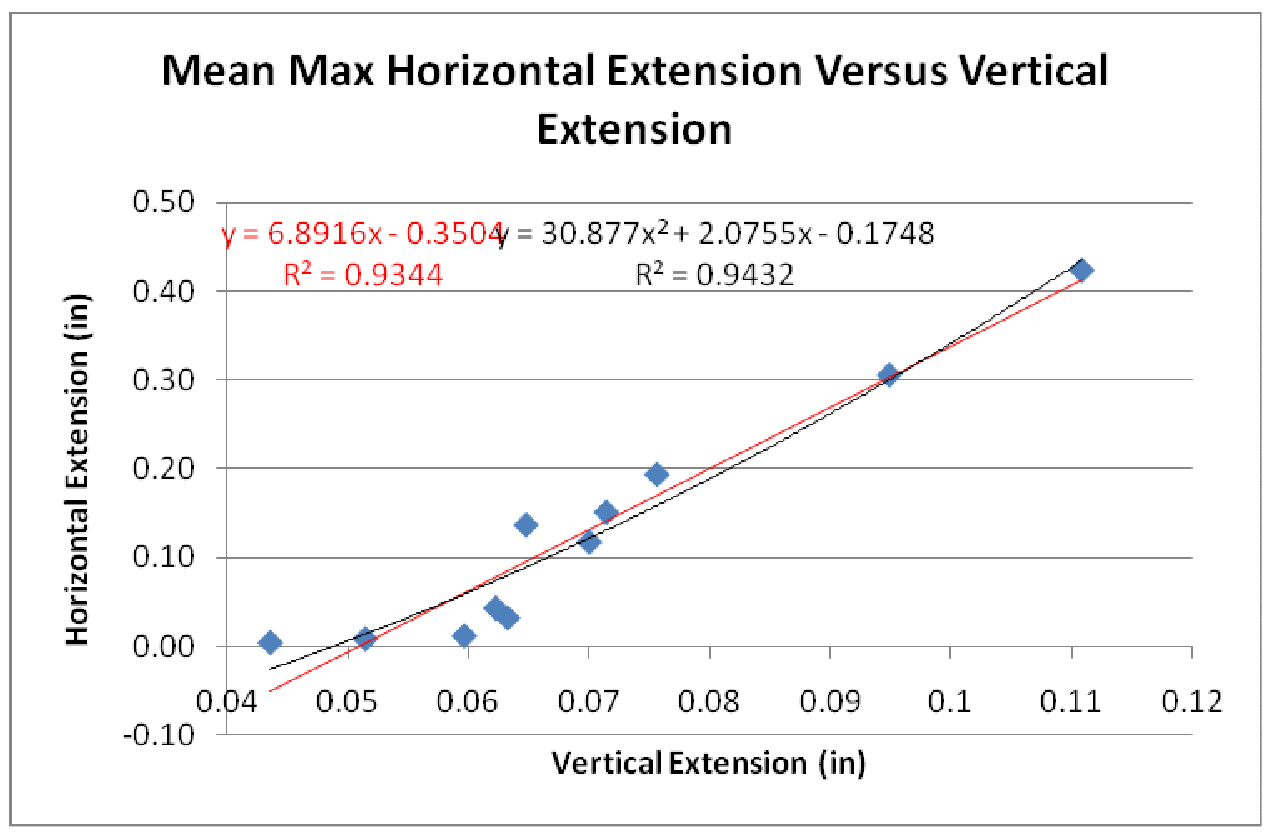

Figure 39: Average Horizontal Extension versus Vertical Extension

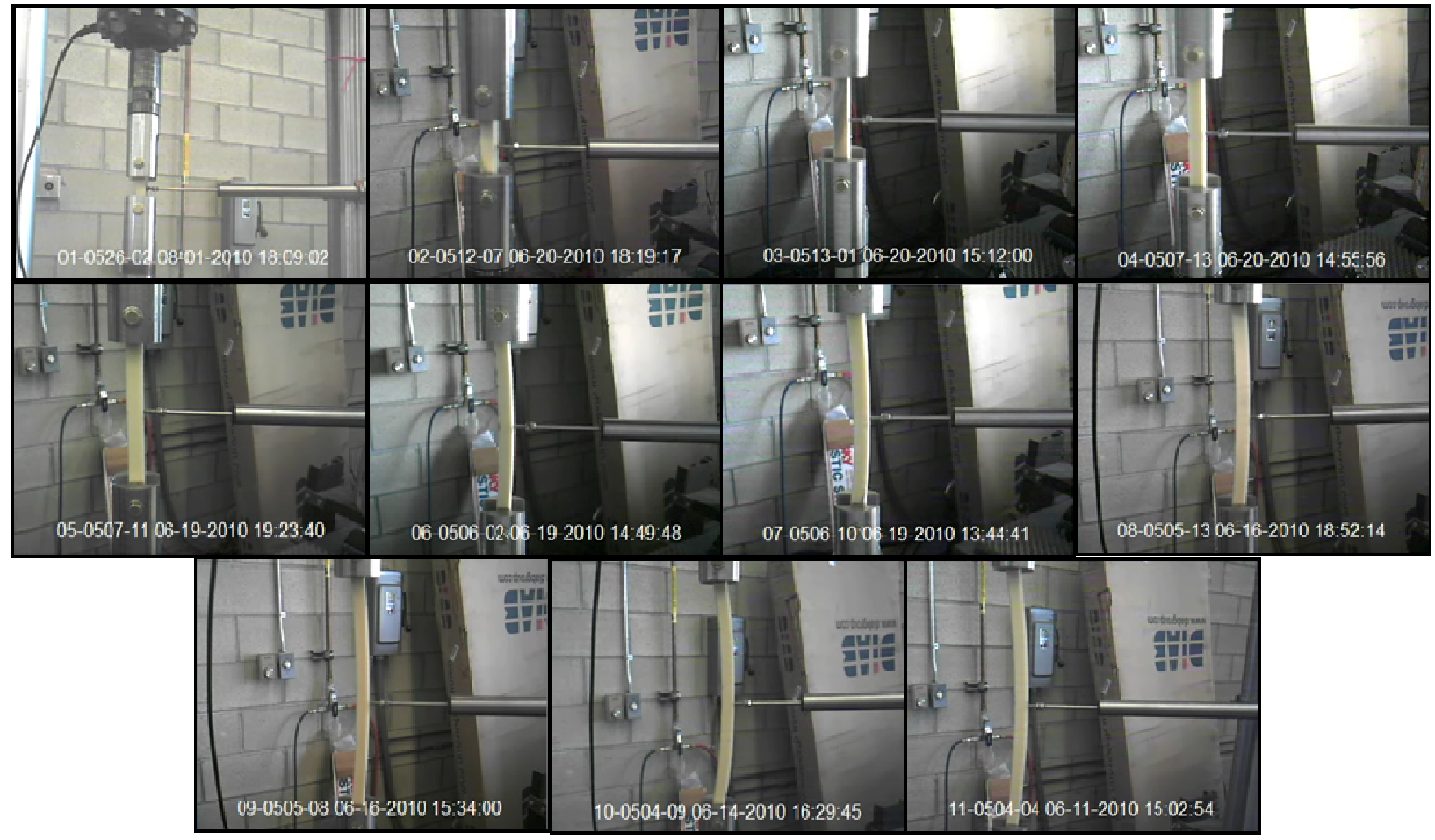

Figure 40: Experimental Test Specimen under In-Plane Displacement

To conclude this section, pictures of the various lengths of test specimens are displayed in Figure 40. These pictures are ordered left to right starting with 1-inch as the starting length and ending with the 11-inch specimen. Note that the material properties, the cross-sectional area, and 
how it is manufactured are the same. The only difference is the length of the test specimens and is subjected to the same in-plane displacement rate. From Figure 40, it can be seen that the longer test specimen lengths experience more horizontal extension and can be achieved at lower loads.

\subsection{Full Solid Cure Trend}

During preliminary testing, the manufacturer information regarding the curing cycle was followed. According to West System 105/206 epoxy labeling, the test specimens would be a full solid cure between 10 to 15 hours at a room temperature of 72 degrees Fahrenheit. The curing temperature for the hardener ranges from approximately between 60 to 95 degrees Fahrenheit.

There is also a note on the labeling that states that the epoxy cures faster in warmer temperature and thicker applications and the opposite with cooler temperatures. The label information is listed in Table 23:

Table 23: Epoxy Label Information

\begin{tabular}{|c|c|c|c|c|c|}
\hline Hardener & Epoxy Uses & $\begin{array}{c}\text { Hardener Temperature } \\
\text { Range (deg F) }\end{array}$ & Pot Life & $\begin{array}{c}\text { Working } \\
\text { Time }\end{array}$ & $\begin{array}{c}\text { Cure to } \\
\text { Solid }\end{array}$ \\
\hline 205 & $\begin{array}{c}\text { Bonding, coating } \\
\text { and fabric } \\
\text { application }\end{array}$ & $40-90$ & $\begin{array}{c}9-12 \\
\text { minutes }\end{array}$ & $\begin{array}{c}60-70 \\
\text { minutes }\end{array}$ & $\begin{array}{c}6-8 \\
\text { hours }\end{array}$ \\
\hline 206 & $\begin{array}{c}\text { Bonding, coating } \\
\text { and fabric } \\
\text { application }\end{array}$ & $60-95$ & $\begin{array}{c}20-25 \\
\text { minutes }\end{array}$ & $\begin{array}{c}90-110 \\
\text { minutes }\end{array}$ & $\begin{array}{c}10-15 \\
\text { hours }\end{array}$ \\
\hline 207 & $\begin{array}{c}\text { Clear coating and } \\
\text { fabric application }\end{array}$ & $60-95$ & $\begin{array}{c}20-25 \\
\text { minutes }\end{array}$ & $\begin{array}{c}90-110 \\
\text { minutes }\end{array}$ & $\begin{array}{c}10-15 \\
\text { hours }\end{array}$ \\
\hline 209 & $\begin{array}{c}\text { Bonding, coating } \\
\text { and fabric } \\
\text { application }\end{array}$ & $65-100+$ & $\begin{array}{c}40-50 \\
\text { minutes }\end{array}$ & $\begin{array}{c}3-4 \\
\text { hours }\end{array}$ & $\begin{array}{c}20-24 \\
\text { hours }\end{array}$ \\
\hline
\end{tabular}

The full solid cure did not actually finish curing after the manufacturer's quote of 10 to 15 hours, but shows no significantly changes between the elastic moduli or the loading capabilities after 13 to 14 days. Therefore, testing of the cured test specimens started on Day 15 and remains valid any time after Day 15 because there are no significant changes in the material properties.

In order to prove that Day 15 and beyond can be used for testing, a test in search of when the epoxy will fully cure with the fiberglass woven roving commenced. The test specimens were 
tested over a 16 day time period with approximately 5 to 6 specimens per day. In order to obtain the resultant data, composite strips made in accordance to ASTM D-3039 standard and placed under tension to determine its material properties. The resultant data over the average 5 to 6 specimens per each day is listed in Table 24. Note that there were only 2 specimens on Day 16 because Day 16 was not in the initial test criteria and was tested because of excess test specimens. This is the reason why Day 16 is a bit higher in the resultant data than the other days and Day 16 is not included when finding trends. There is also no data for Day 1 because the manufacturing of the test specimens required a day of layup and curing.

Table 24: Tensile Material Properties per Each Day

\begin{tabular}{|c|c|c|c|c|c|}
\hline Day & $\begin{array}{c}\text { Average } \\
\text { Max Load }\end{array}$ & $\begin{array}{c}\text { Average Max } \\
\text { Tensile Stress }\end{array}$ & $\begin{array}{c}\text { Average Extension at } \\
\text { Max Load }\end{array}$ & $\begin{array}{c}\text { Vertical } \\
\text { Strain }\end{array}$ & $\begin{array}{c}\text { Average } \\
\text { Modulus }\end{array}$ \\
\hline$\#$ & lbf & psi & in & in & Psi \\
\hline 2 & $1.5608 \mathrm{E}+03$ & $3.8063 \mathrm{E}+04$ & 0.0977 & 0.0199 & $1.8438 \mathrm{E}+06$ \\
\hline 3 & $1.6500 \mathrm{E}+03$ & $3.8490 \mathrm{E}+04$ & 0.0968 & 0.0197 & $1.9404 \mathrm{E}+06$ \\
\hline 4 & $1.6795 \mathrm{E}+03$ & $3.6786 \mathrm{E}+04$ & 0.0955 & 0.0194 & $1.8130 \mathrm{E}+06$ \\
\hline 5 & $1.6482 \mathrm{E}+03$ & $3.6886 \mathrm{E}+04$ & 0.0915 & 0.0186 & $1.8656 \mathrm{E}+06$ \\
\hline 6 & $1.6052 \mathrm{E}+03$ & $3.6312 \mathrm{E}+04$ & 0.0917 & 0.0185 & $1.8297 \mathrm{E}+06$ \\
\hline 7 & $1.6256 \mathrm{E}+03$ & $3.6695 \mathrm{E}+04$ & 0.0880 & 0.0177 & $1.9089 \mathrm{E}+06$ \\
\hline 8 & $1.7416 \mathrm{E}+03$ & $4.0080 \mathrm{E}+04$ & 0.0914 & 0.0186 & $1.9964 \mathrm{E}+06$ \\
\hline 9 & $1.6927 \mathrm{E}+03$ & $3.9878 \mathrm{E}+04$ & 0.0919 & 0.0188 & $1.9730 \mathrm{E}+06$ \\
\hline 10 & $1.7772 \mathrm{E}+03$ & $4.0044 \mathrm{E}+04$ & 0.0902 & 0.0184 & $2.0175 \mathrm{E}+06$ \\
\hline 11 & $1.6036 \mathrm{E}+03$ & $3.7855 \mathrm{E}+04$ & 0.0898 & 0.0183 & $1.9123 \mathrm{E}+06$ \\
\hline 12 & $1.8756 \mathrm{E}+03$ & $4.0641 \mathrm{E}+04$ & 0.0951 & 0.0191 & $1.9969 \mathrm{E}+06$ \\
\hline 13 & $1.7340 \mathrm{E}+03$ & $3.7549 \mathrm{E}+04$ & 0.0909 & 0.0184 & $1.9168 \mathrm{E}+06$ \\
\hline 14 & $1.7941 \mathrm{E}+03$ & $3.8833 \mathrm{E}+04$ & 0.0927 & 0.0187 & $1.9181 \mathrm{E}+06$ \\
\hline 15 & $1.7381 \mathrm{E}+03$ & $3.8122 \mathrm{E}+04$ & 0.0895 & 0.0179 & $1.9459 \mathrm{E}+06$ \\
\hline 16 & $1.9188 \mathrm{E}+03$ & $4.0803 \mathrm{E}+04$ & 0.0923 & 0.0186 & $2.0117 \mathrm{E}+06$ \\
\hline
\end{tabular}

The resultant data regarding the loading capabilities of the test specimens compared over a 16 day time period is detailed in Figure 41. With the initial start at Day 2, the graph shows that the loading capability of the test specimens increases over time until it begins to plateau around Day 14. There are two trend lines that are able to represent the data. First the exponential fitted trend line coded with the color red and has a coefficient of determination of $45.6 \%$. The other is 
the parabolic fitted trend line coded with the color black and has a coefficient of determination of 45.7\%. The coefficient of determination for this experiment is low due to the limited number of test specimens per day. The coefficient of determination would increase if there were more specimens to test, but with limited resources, testing 5 to 6 specimens per case seems reasonable to obtain the trends.

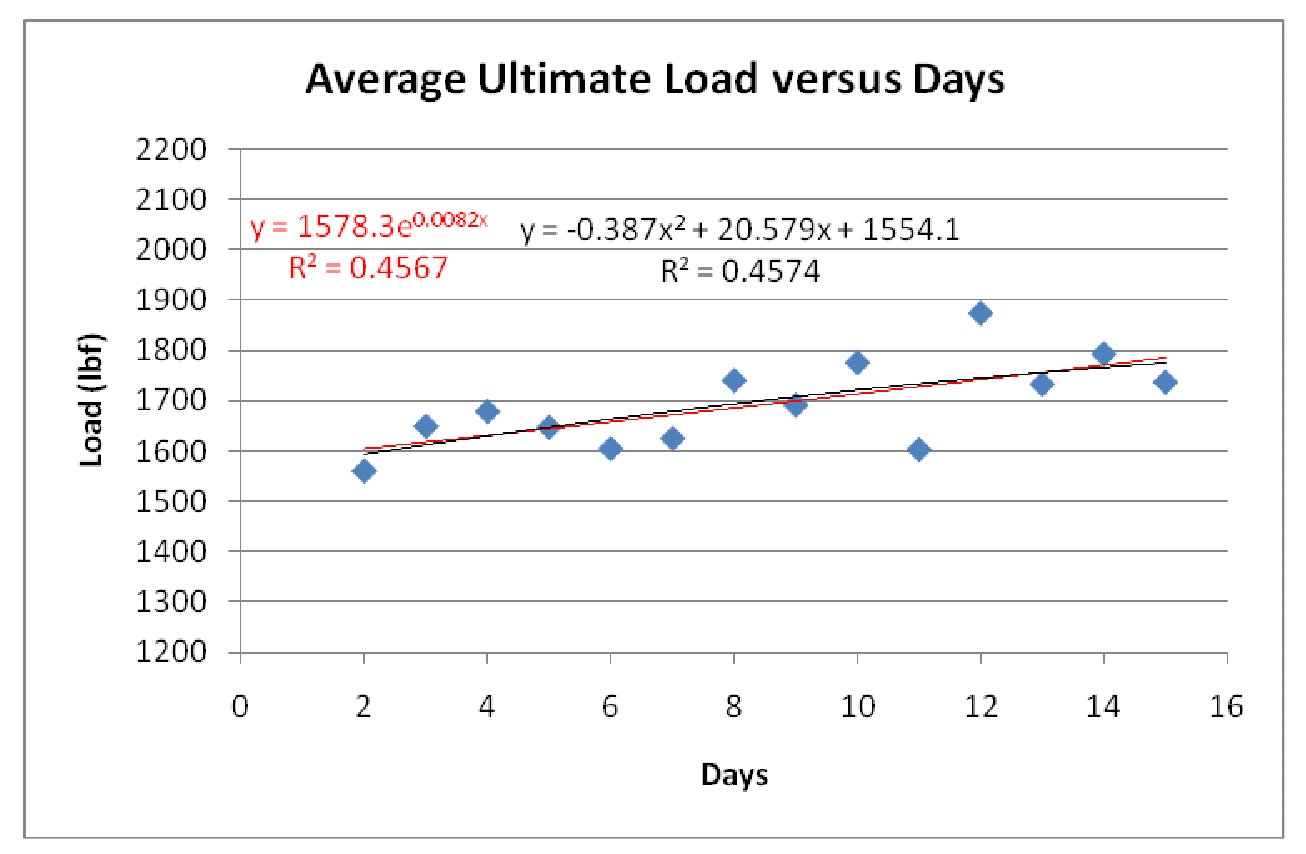

Figure 41: Average Ultimate Load versus Days

Similarly, the ultimate tensile stress versus the various days is detailed in Figure 42. The trend is similar to that found in Figure 41 where it shows no significant changes around Day 14 and is represented by the parabolic trend line and equation listed in Figure 42 . 


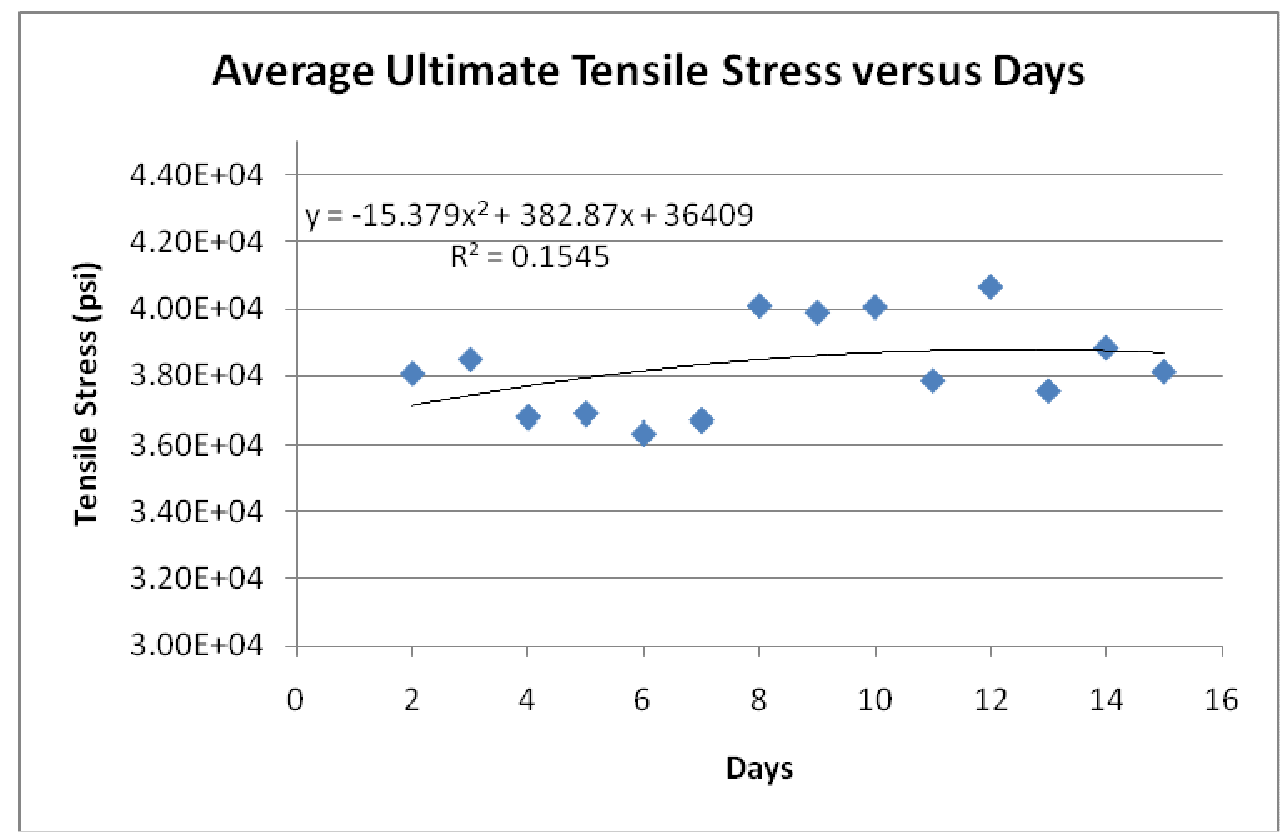

Figure 42: Average Ultimate Tensile Stress versus Days

Figure 43 shows the average maximum vertical extension the test specimens experience over the 16 day time period and shows no significant changes after Day 12 . The decrease in the extension can be attributed to the increase in the stiffness of the material as it becomes fully cured. The specimens would break at shorter extensions over a longer time period before it began to plateau. The longer elongation during the earlier times details a more malleable material. The parabolic trend line is best fitted to the plotted data with a coefficient of determination of $53.7 \%$ while the logarithmic trend line has a coefficient of 50.0\%. It can be believed that the parabolic trend line provided a good fit up and trails off after its lowest point. The logarithmic trend line seems to be more believable because material properties of the test specimens would become constant after an amount of time. The logarithmic trend line and equation is coded by the red color and the parabolic trend line is represented by the black color. The issue with these trend lines can be accounted for in the limited amount of test specimens as discussed before. 


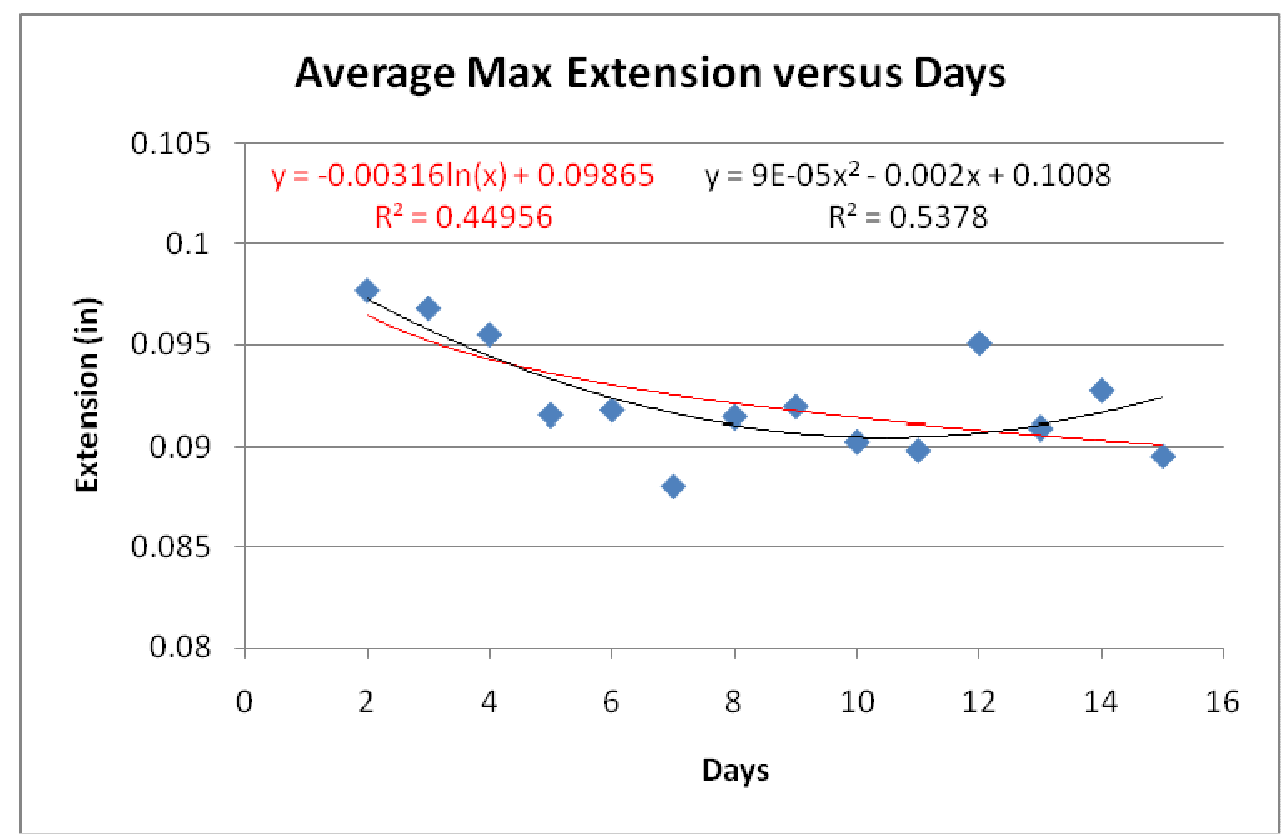

Figure 43: Average Maximum Extension versus Days

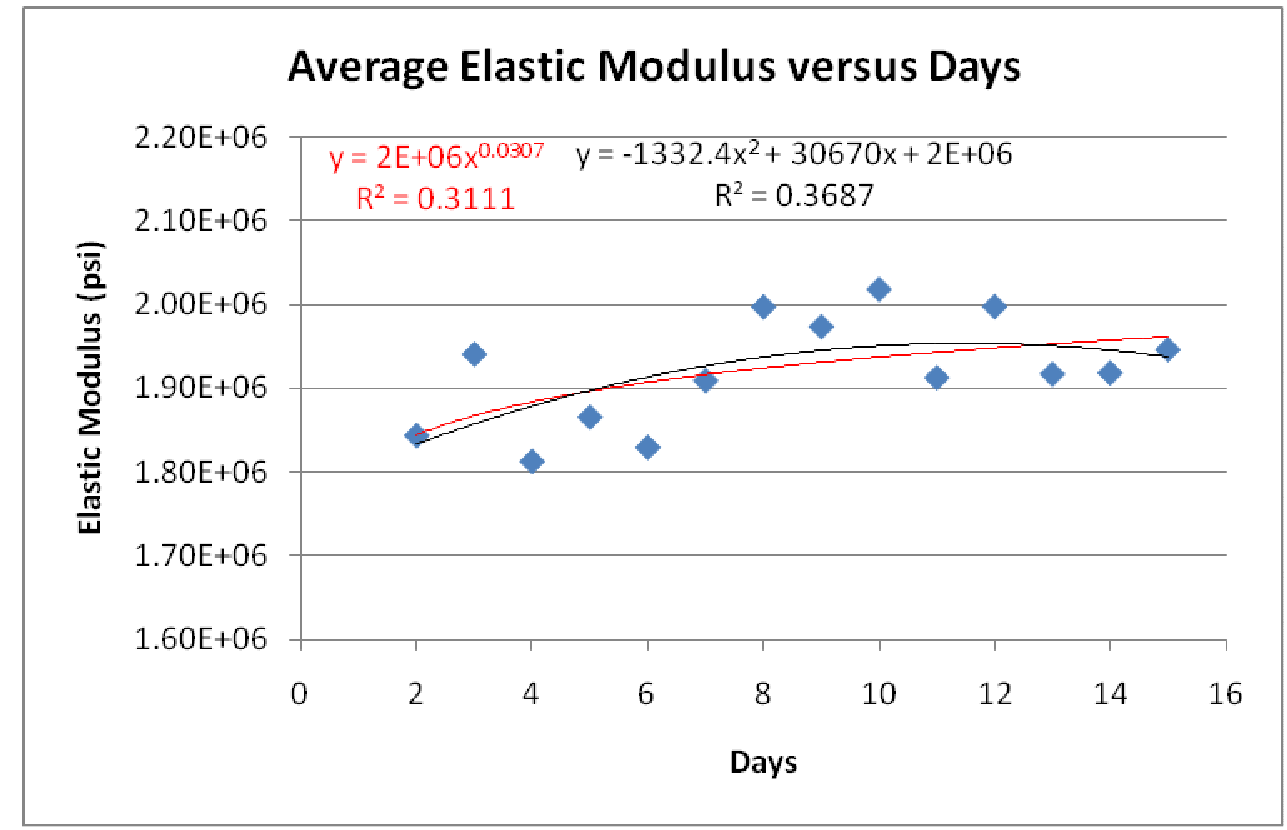

Figure 44: Average Elastic Modulus versus Days

The average elastic modulus of the test specimens begins to plateau around Day 13 to Day 14 and is best represented by the parabolic trend line. Again, the parabolic trend line is best fitted to a certain point before it trails off. The parabolic trend line and equation is coded with the black color in Figure 44. The other trend line has an exponential fit and, similar to the logarithmic 
trend line in Figure 42, provides a more reasonable fit because the material properties of the specimens become constant after a certain amount of time.

Day 15 is the break point when testing begins and it is validated that no significant changes occurred in the studied material properties.

There were also some differences in how the failure happened when the specimens were tested. Detailed in Figure 45, it shows a couple different failure modes the specimens go through. Depending on when the specimens were made, the failure mode seen in the top image of Figure 45 is more prevalent until the 12th day when the test specimens mature to have a failure mode defined by the bottom image. The top image was quite an interesting one when the regions of delamination started to pop up before the fracture occurred. This usually means that the fibers are carrying the loads and the matrix did not support them well enough. As for the bottom image, the fracture occurs and then the region around the fracture began to delaminate.
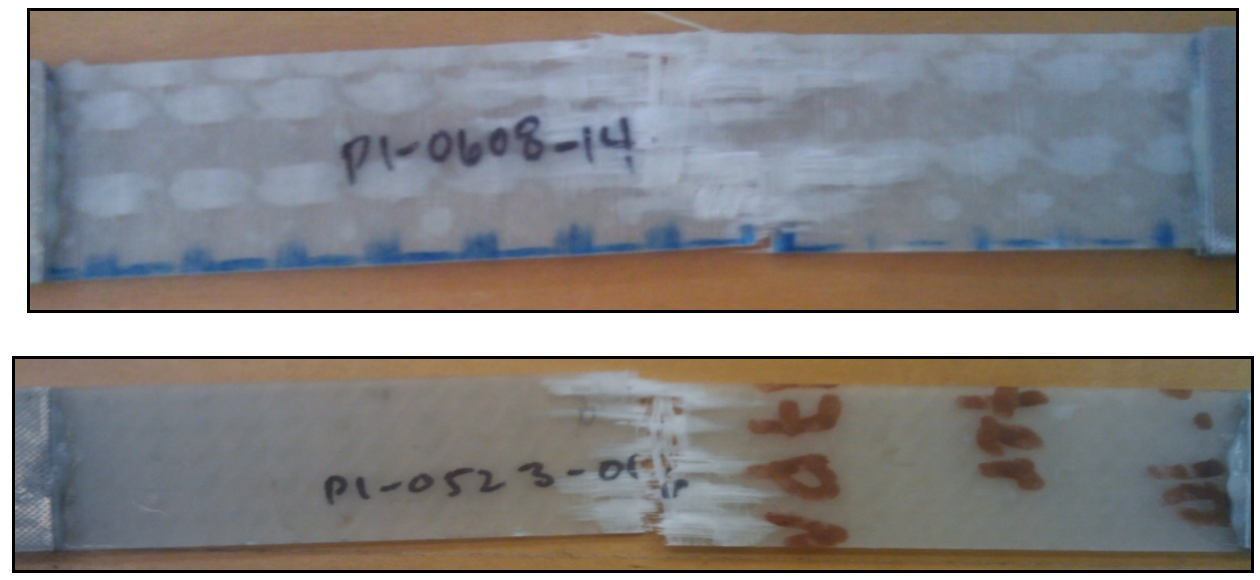

Figure 45: Different Failure Modes

\subsection{Manufacturing Process Selection}

There are two particular manufacturing process used throughout this project. One is known as vacuum resin infusion (VRI) method and the other is the hand "wet layup" curing method. These two methods were chosen because it is used fairly often in the boating and aerospace industries, its manufacturing process is fairly simple, it is cheaper in cost to 
manufacture than pre-impregnated composite material, and it provides a consistency similar to that of pre-impregnated composite material.

Though these manufacturing processes have favorable benefits, there are also limitations to them as well. Some of the limitations include a lot of components during the layup process especially for VRI and epoxy being exposed to air contaminates for the wet layup method.

The hand cure technique is used throughout many fields that use composites and they are mainly used for small-scale repairs and simple forming of the composite structure. The hand cure technique is a method used to manually infuse the epoxy into the fiber of the composite material prior to forming the structure. Usually the infused composite structure is vacuumed to provide better matrix consistency across the structure and to secure the shape of the structure. That is why this particular technique is used for the manufacturing of the arrestment key. A trial layup using the VRI became ineffective because the infused epoxy did not have enough suction to pull across all 22 keys in its mold. Due to this ineffectiveness, the hand layup was the optimal choice to make these arrestment keys.

As for the VRI technique: consistency, avoidance of contamination, and cost are the driving forces in building all of these composite sandwich structures. There were more than 20 different configurations to do and they can be quite costly. Also, this particular technique removes many worries about consistency because of the uniform distribution of pressure across the vacuumed environment. Air contaminates were eliminated so that they do not affect the structural integrity.

\subsection{Testing Apparatus Design}

The testing apparatus has gone through several design iterations in order to overcome certain limitations and to obtain certain conditions to provide the correct environment for the composite test specimens. These are three different iterations that the testing apparatus went through and coincide with the test specimen's evolution. 


\subsection{1 $\quad 1^{\text {st }}$ Generation Testing Apparatus Design}

The first test specimen started off with a simple design where there were no wood boundary core, no holes in the structure, and no complicated testing apparatus. The test specimen was a simple composite sandwich structure which consisted of just the e-glass skin and the foam core and nothing else. The test apparatus to support this structure and to have the structure placed under buckling loading was a tee-shaped jig where the edgewise of the specimen would lie on the top of the tee-shaped jigs and be displaced by the Instron machine. The tee-shaped jigs are mounted to the wedge grips that came with the Instron machine.

There were a few major issues that arose from this design. The first is that the testing apparatus causes edge bearing to happen and fails at the edge before the test specimens can even buckle properly. The second is that the tee-shaped jig required perfection alignment with the Instron machine and the test specimens in order to avoid angled loading. These issues drove a need for a new design of the test specimen and the test jigs. An example of this testing apparatus can be seen in Figure 46. 


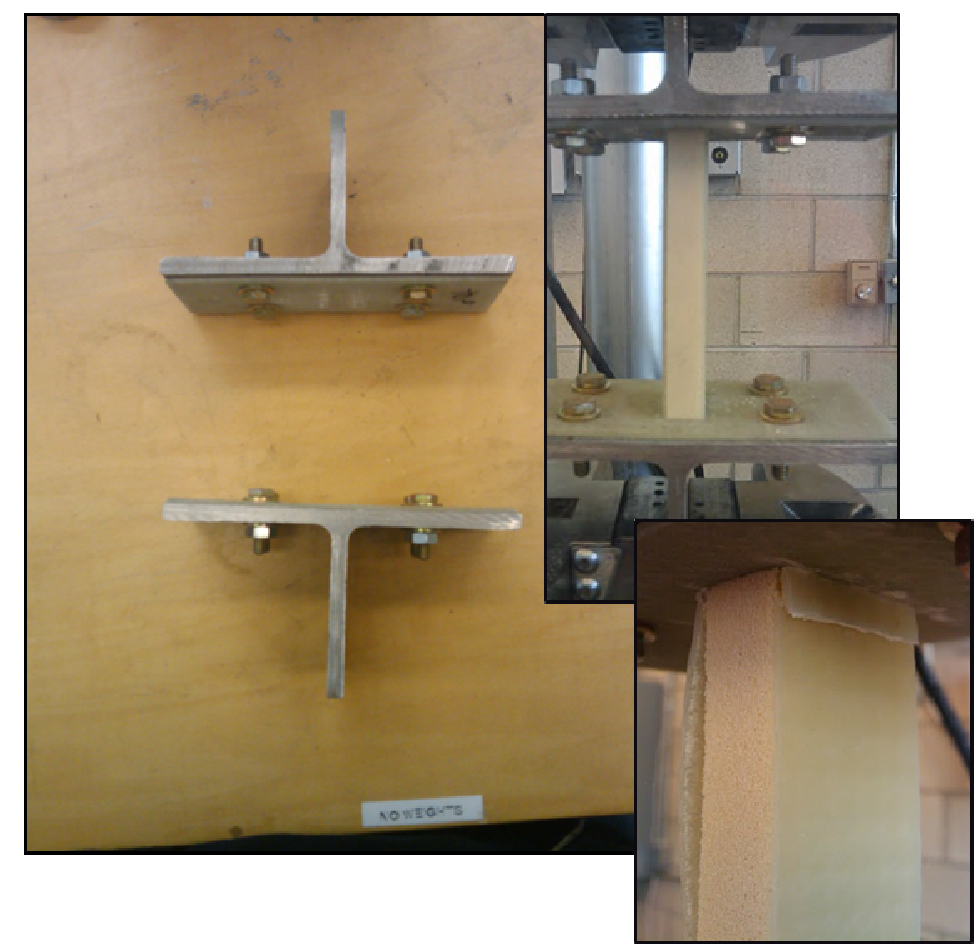

Figure 46: Tee-Shaped Jig and Its Issues

\subsection{2 $\quad 2^{\text {nd }}$ Generation Testing Apparatus Design}

The second iteration of the test apparatus improved in order to overcome the issues seen in the first iteration. This particular apparatus utilizes a pinned-pinned condition in order to remove the edge bearing issue seen in the first design. It also provides an update of the test specimen were a wood core is introduced. An example of the test jig is displayed in Figure 47. 


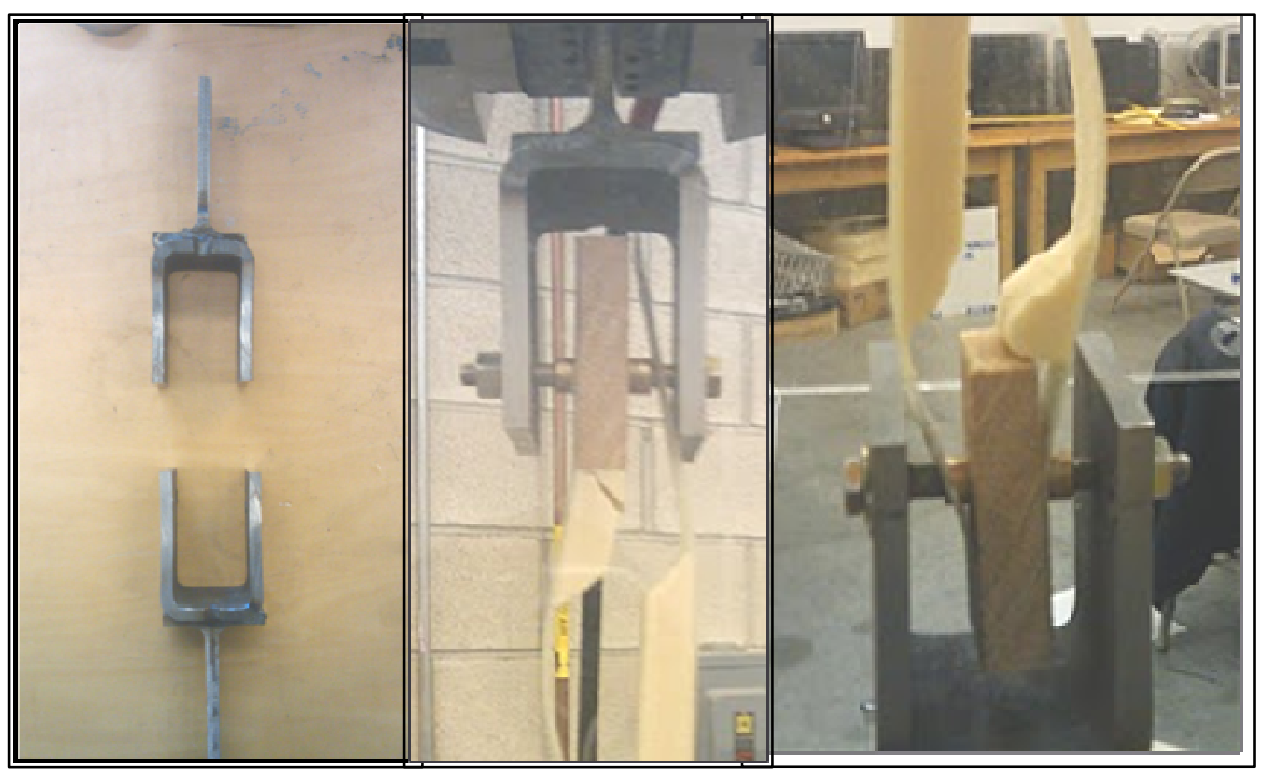

Figure 47: Second Iteration Testing Apparatus and Its Issues

This particular design did eliminate the previous errors found in the first design, but led to a few others. The issue found in this design is with the alignment of the testing jigs to the wedge grips that caused angled loading to happen frequently. On top of that, the moment of inertia in the direction of the buckling of the test specimen was off. Thus a third generation of the test specimen and testing apparatus was created.

\subsection{3 $\quad 3^{\text {rd }}$ Generation Testing Apparatus Design}

The last generation for the testing apparatus did the trick. It was able to produce a pure buckling of the longer test specimens and misalignment issue when second-guessing how the test jigs were to be mounted to the wedge grips.

An example of the third and last generation is displayed in Figure 48. 


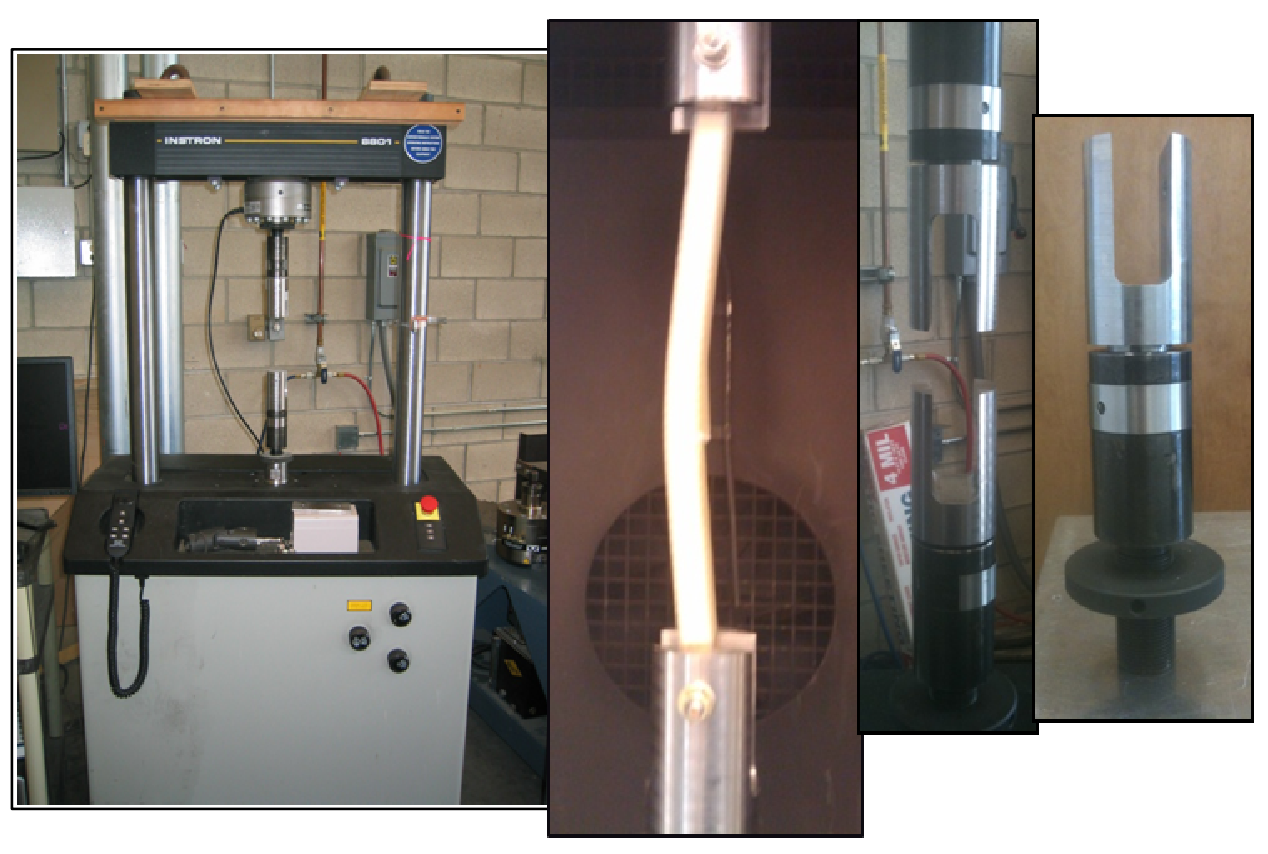

Figure 48: Third Generation Testing Apparatus

This generation of the test apparatus removed the alignment issue by removing the wedge grips themselves and mounting the testing apparatus directly to the Instron machine connecting it to the displacement rod and load cell. In order to achieve the buckling, the pinned-pinned boundary had to be rotated 90 degrees and was supported by only the wood cores. This generation of the testing apparatus was used for all the different configurations. The first generation testing apparatus was only used for determining several of the mechanical characteristics under compressive loading.

\subsection{Hole Drilling Optimization.}

What was discovered when trying to drill the pivot holes through the composite skin or even the wood core alone was that there were a lot of nonconformity that arises during the manufacturing process. With previous research and actually testing of this, the problem with drilling into fibrous composite material is that burrs, delamination, and even heat damage can affect the structural integrity at these hole locations.

The first attempt was to use a standard drill bit that can easily be found. The issue with these types of drill bits is the amount of teeth they have. The use of this standard bit shows burrs 
at slow feed rate and delamination at high feed rate. This was not acceptable for the structure because if loaded, it put a high eccentric stress concentration at the hole locations.

The second drilling method uses a rotary sander to form the holes. The issue with this is that the hole would be inconsistent and hole diameters were larger than what the sanding bit specified.

This issue led to the use of a tile drill bit that was shaped similar to a spade. At slow feed rate and moderate revolution rate, there were minimal signs of burrs and no signs of delamination. This method was used to make all the required holes for the different configurations. Examples of the tile drill bit in action can be seen in Figure 49.

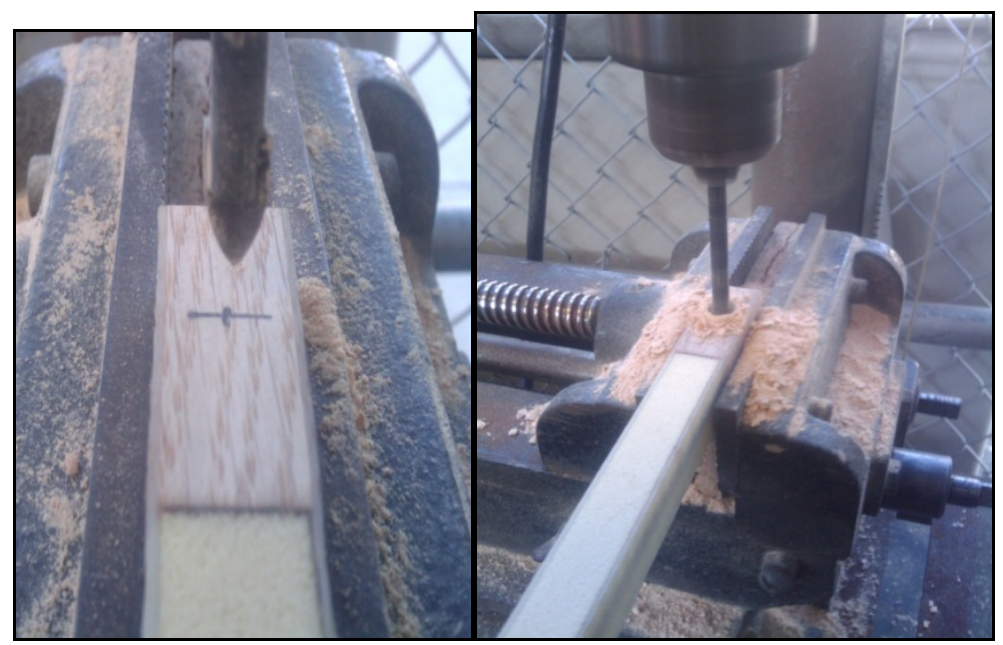

Figure 49: Examples of Tile Bit in Use

\subsection{Wood Boundary Core Issues and Resolution}

As discussed before, the wood boundary cores are used to support the support when it is loaded. There was an issue that came up during the first use of these wood cores. With in-plane loading, the hole would be oblong and crack propagation begins at the hole location and travels through the whole structure. This initial failure at the boundary was not acceptable.

The issue was the wood grain orientation. The grain was initially placed perpendicular to the in-plane load vector. With the rotation of the wood core such that the grains were parallel to 
the load vector, there was no sign of holes being oblong and cracks did not start at the hole locations. Examples of these issues can be seen in Figure 50.

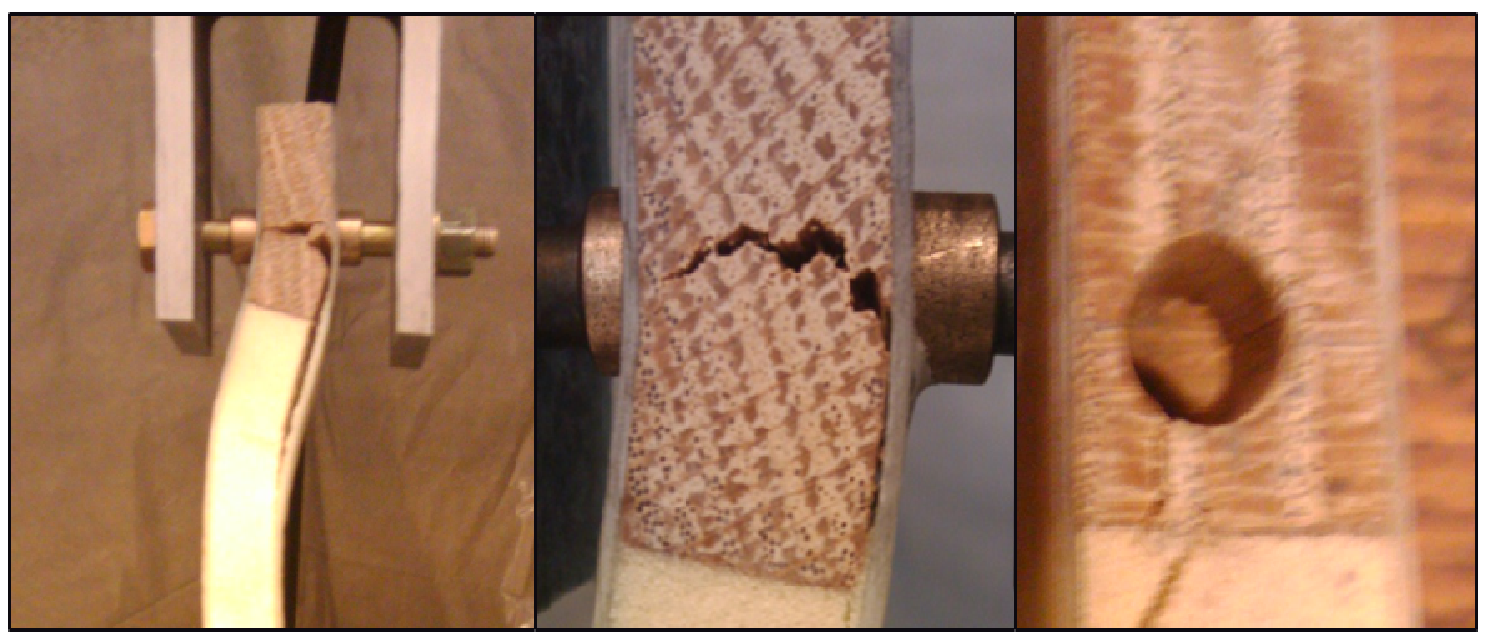

Figure 50: Examples of Wood Boundary Core Issues 


\section{MANUFACTURING, PREPPING, AND TESTING PROCEDURES}

\subsection{Manufacturing and Prepping Procedures}

This section will discuss the manufacturing and build up of all the different test specimens which include the composite strips to test for material properties to the composite sandwich structure with all its different configurations. The manufacturing and prepping procedures will go into details starting with the initial cut of the foam core to the resin infusion process.

\subsubsection{Foam Preparation}

The preparation of the foam starts off with obtaining the foam sheets from the manufacturer which is dimensioned 8 feet long by 4 feet by 0.5 inches in thickness. With the limited width size of the supplied wood and how much pressure the vacuum can provide once vacuumed and epoxy is pulled, the foam sheets are cut down to be 11 inches by 9.75 inches by 0.5 inches to fit these parameters. A table saw is used to cut the 8 feet by 4 feet foam sheets to the correct measurements and requires a few people to maintain the steadiness of the foam sheet while it is fed through the table saw. A picture of the cutting process can be seen in Figure 51.

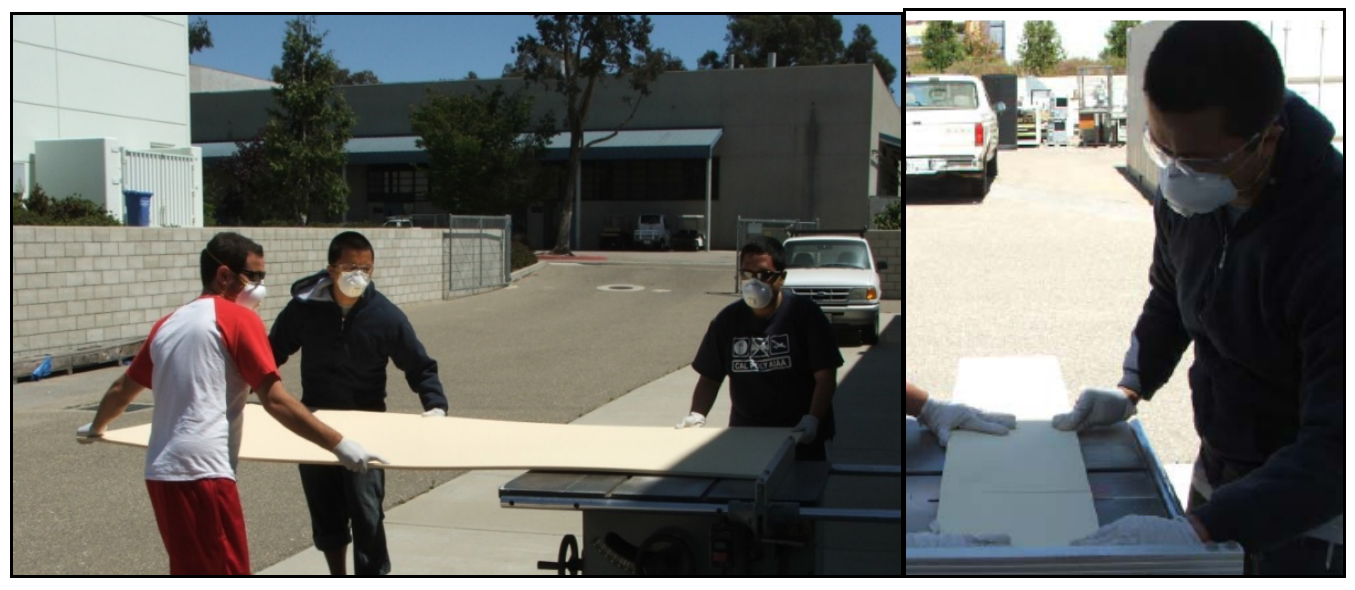

Figure 51: Foam Prepping 
With cutting these foam sheets, well-being of each individual is a priority. Safety goggles, particle-based masks, and latex gloves are used to protect the users at all times during the foam prepping process. Also, conscious awareness of a rotating table saw blade is paramount. Inhalation of foam particles or from the table saw blade can lead to major bodily injuries and health issues, so safety is always a priority in all prepping processes.

Approximately 40 foam plates were cut down to size and use to manufacture all the test specimens used in this thesis project.

\subsubsection{Foam Groove Milling Prep}

Several of the configurations of test specimens require the foam to be milled and be able to support the arrestment keys. Therefore, with the correct cut down dimensions of the foam plates, the plate is manually milled using a 0.25 -inch ball end mill bit to create all the necessary grooves as was discussed previously in the specimen design section of this report. An example of the foam milling is displayed in Figure 52.

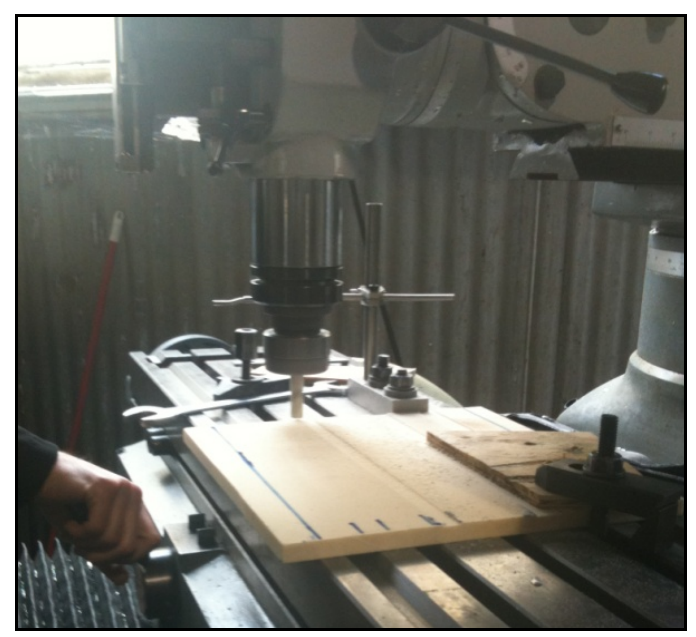

Figure 52: Foam Milling

\subsubsection{Fiber Preparation}

The preparation of composite fiber starts with the obtained roll of fiberglass woven roving from a distributor. It is important to note the significance of contaminate prevention and fiber alignment when cutting these sheets. Contaminates can also be anything from dust particles 
to small residues from a previous layup and can affect the structural integrity and the results.

Precautions such as covering the fiberglass roll and storing it away from large sources of motion are some of the ways to minimize these errors. Fiber alignment is specifically targeting the cutting of the fiberglass into sheets where a rotary blade is used to cut the fiberglass sheet rather than a box cutter. The roll of fiberglass roving starts off with a cut down of the fiberglass roll into sheets that are slightly larger than the overall foam plate with its wood boundaries. The actual dimensions are approximately 16 inches by 12 inches, which provides a 1 -inch margin around the whole plate configuration. When the rotary cutters are worn, the blades are scrapped and replaced to avoid tussle-like edges. In order to avoid manufacturing delays, the majority of these sheets are cut down to size prior to all layups. This reduces the manufacturing time and easy integration. There are 4 of these sheets used for each layup and are specifically used as the composite skin of the structure. An example of the cut down sheet is displayed in Figure 53.

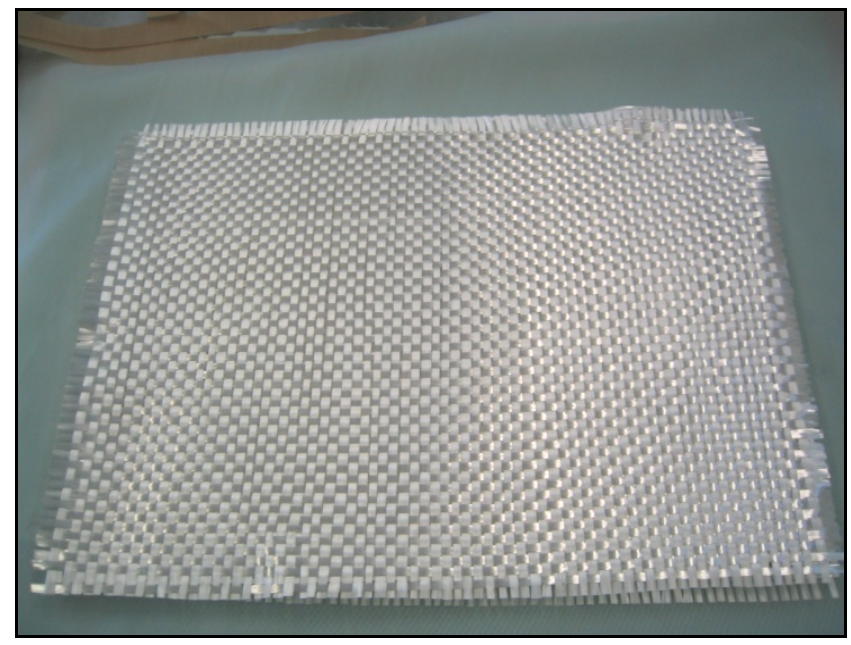

Figure 53: Fiber Prep

\subsubsection{Delamination Arrestment Key Preparation}

The same fiberglass woven roving is used during the prep work for the arrestment key. Instead of cutting it down to form sheets, the fiberglass strands are pulled off of the weave and grouped together into a bundle of 17 strands. The 17 strand count are derived from how many of these strands can fit into the grooves on the mold and be manually capable of maintaining the 
cross-sectional shape of the arrestment key throughout the layup. The length of the strands has to be a bit longer than 12 inches to provide excess length for trimming and fitting perfectly into the composite sandwich structure. The fiberglass strands pulled off the weave can be seen in Figure 54.

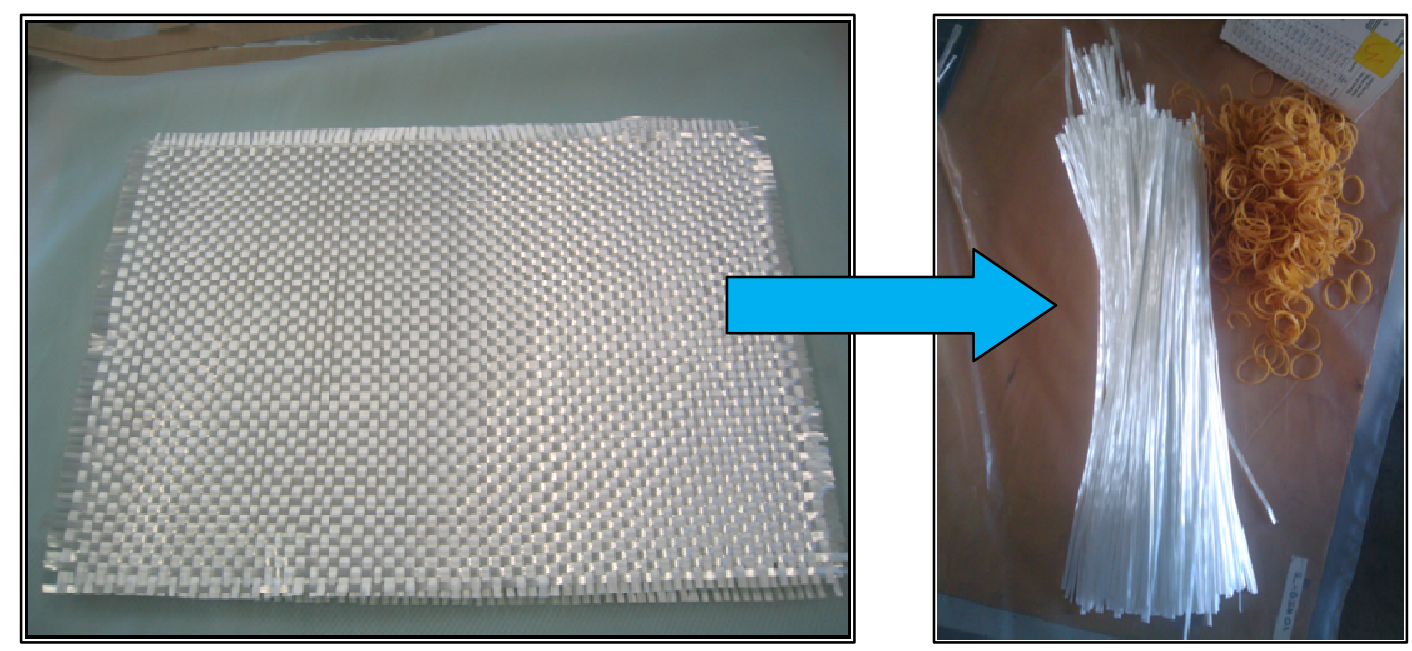

Figure 54: Obtaining the Fiberglass Strands

Once the fiberglass strands are grouped in a set of 17 strands, the strands are held together with a rubber band on one end. It does not need to be a rubber band; it can be anything that holds the strands together and not impact the mechanical properties of the keys. This bundling process can be seen in Figure 55. 


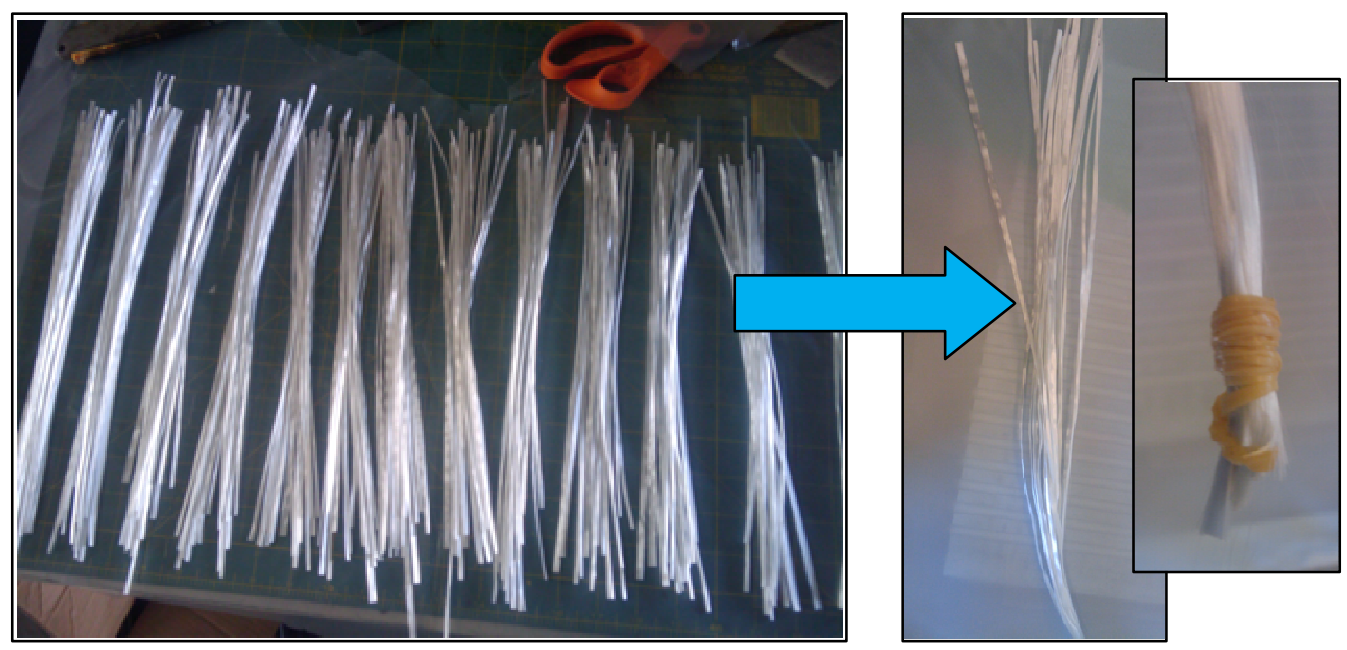

Figure 55: Bundling of the Fiberglass Strands

With a cup of 5 to 1 ratio of the West System 105/205 epoxy, the sets of fiberglass strands are then infused manually to where there is an excess amount dripping when holding the set up vertically. The excess epoxy will later be removed per pressurized vacuumed system. It is important to have enough epoxy rather than end up with dry fiberglass in the delamination arrestment key. Make sure that all parts of the sets are wetted with the epoxy. Once the set of fiberglass strands have enough epoxy, it is placed into the grooves on the mold. The mold is first coated with a non-stick wax and is made of Aluminum AL 2024. The surfaces and grooves of the mold are clean before every use to avoid any contaminates getting into the keys during the manufacturing process. The infused set of strands placed on the mold can be seen in Figure 56.

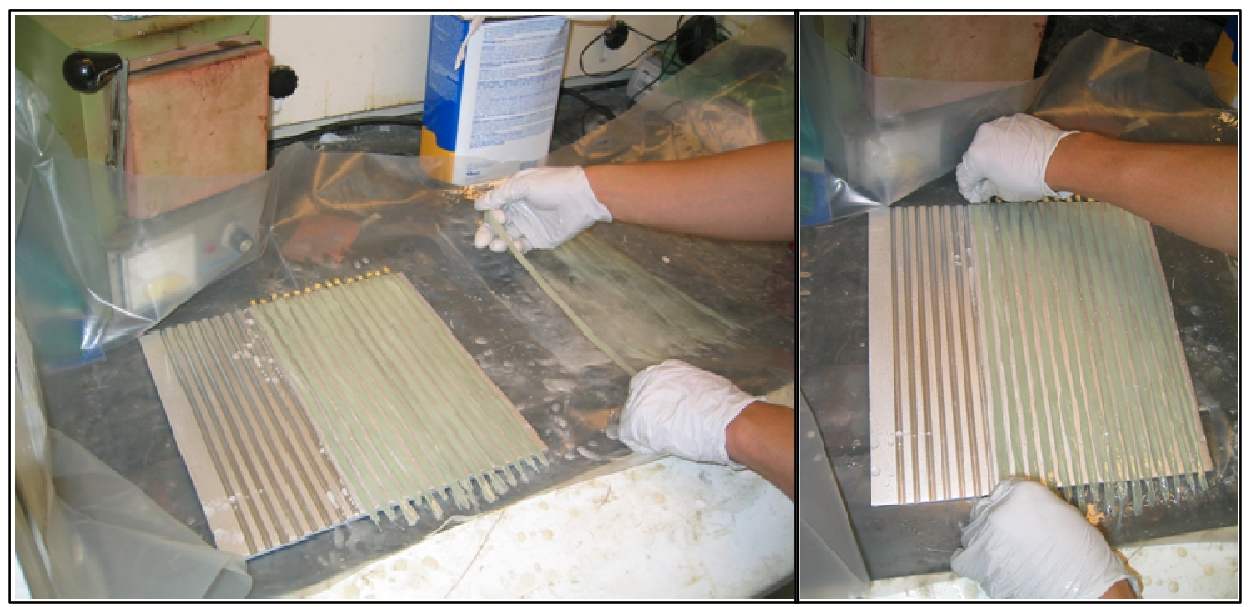




\section{Figure 56: Placing into the Mold}

After all the sets of fiberglass strands are put in place, the whole mold with the premature keys is then placed into a vacuum bag and sealed. The vacuum bag has only one output to pull to create the necessary pressure on the keys and remove any unused epoxy in the vacuumed environment. This is displayed in Figure 57.

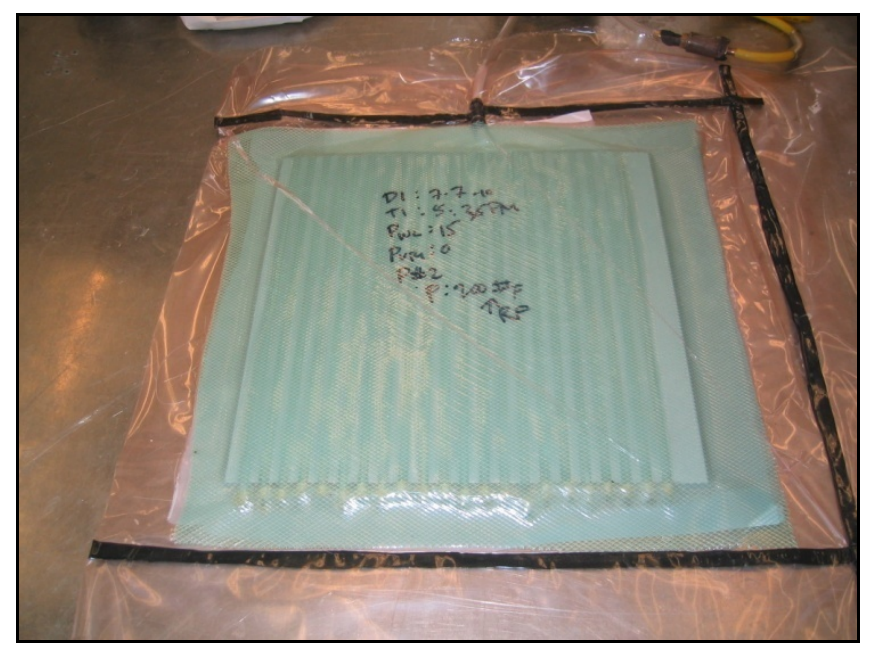

Figure 57: The Vacuumed Mold and Premature Keys

To add additional support for the shape of the delamination arrestment keys, a uniform plate and 200 pounds of additional weight is placed on top of the vacuumed structure. This is done because the pressure in the vacuumed environment would otherwise leave a thin layer of epoxy through the part. In order to help remove the excess epoxy, the weights are added and also provide a flat backing for the shape of the keys. Figure 58 provides a better look into how the weights are placed. 


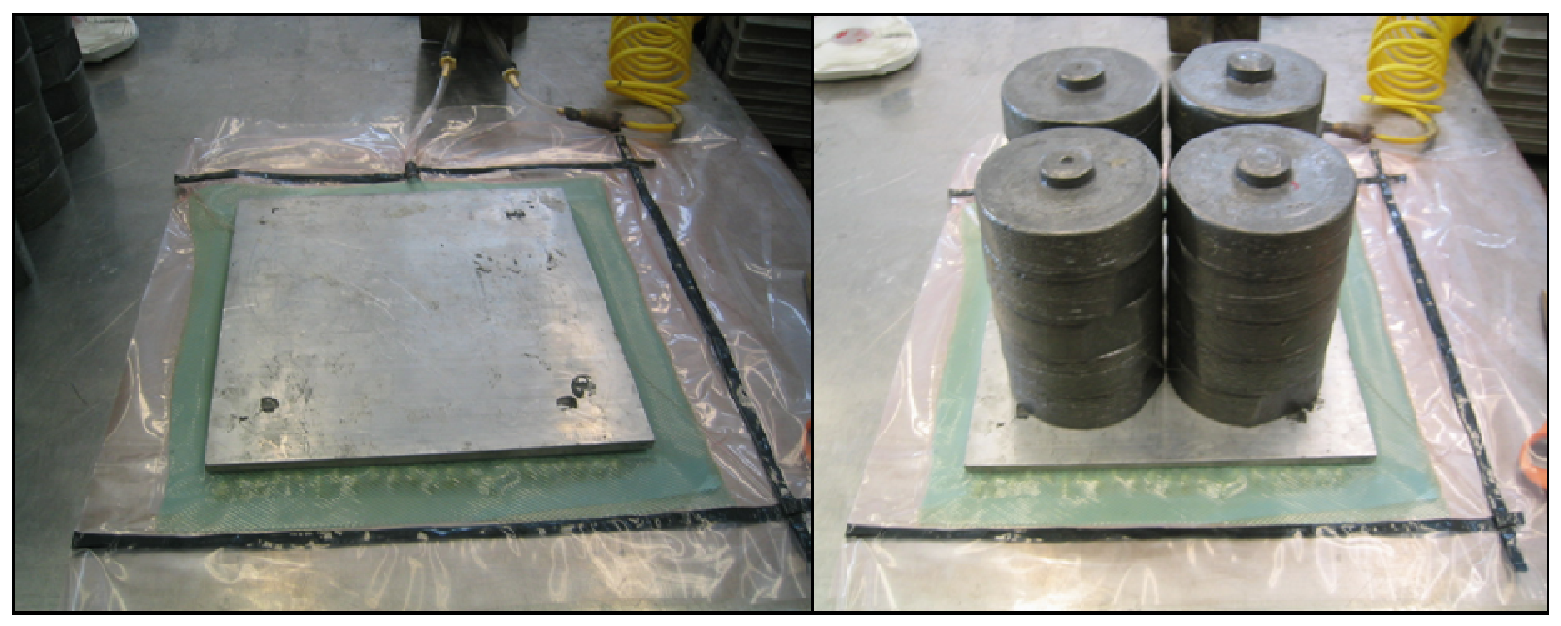

Figure 58: Weights used on Mold

After the curing of the keys is completed, the structure is then taken out of the mold. At this point, the rubber band section of the layup is later cut off to remove what does not belong in the composite sandwich structure. The keys are cut down along its length to reduce most of the excess materials before being placed back into the mold where the keys are then sanded down to the correct dimensions. A picture of the sanding process is displayed in Figure 59.

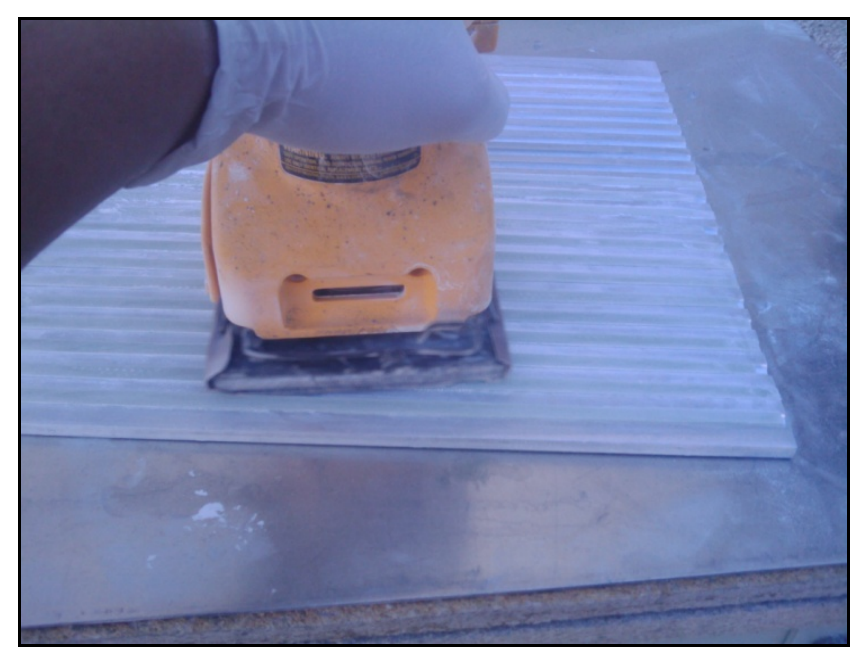

Figure 59: Sanding of the Keys

The keys would look something like Figure 60 after the sanding and cleaning of the keys is completed. The cleaning of the key is done with water and nothing else. All residues on the exterior of the keys are removed, and the keys are stored in a clean environment. 


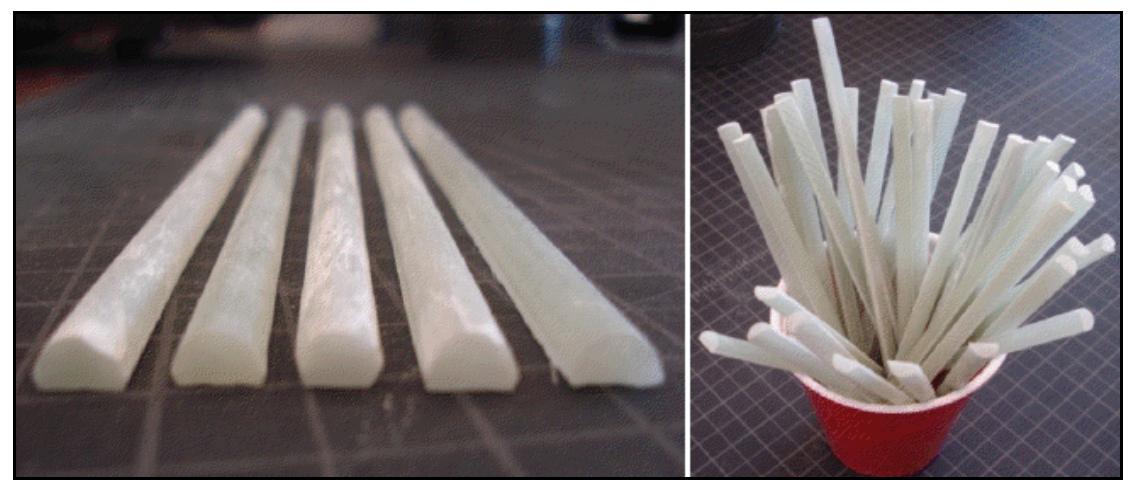

Figure 60: The Keys Completed

\subsubsection{Composite Strip Preparation for Mechanical Properties Testing}

The composite strips used to test for the tensile and compression properties were discussed before in the previous chapter in regards to ASTM D-3039 and ASTM D-3410 standard methods for the design, manufacturing, and testing of these specimens.

The manufacturing of these specimens come from the VRI method where two layers of the fiberglass woven roving is epoxy is infused into the fibers by vacuum suction. The plate that comes from the VRI layup is then cut down to the correct dimensions and the appropriate tabs are glued onto the test specimens using 3M Scotch-Weld Epoxy Adhesive DP-460 with its 3M EPX Plus II Applicator. Note that the fiberglass regions where the tabs will be placed are lightly sanded with 80 grit sandpaper to provide better surface adhesion, and the tabs are also roughened.

Once all four tabs are placed at the appropriate locations per ASTM D-3039 and ASTM D-3410 standard methods, a uniform plate and weights are placed on the test specimens to provide a uniform spread of the Scotch-Weld adhesive. After a day of letting the specimens cure, they are ready for testing. An example of the outcome after manufacturing is displayed in Figure 61.

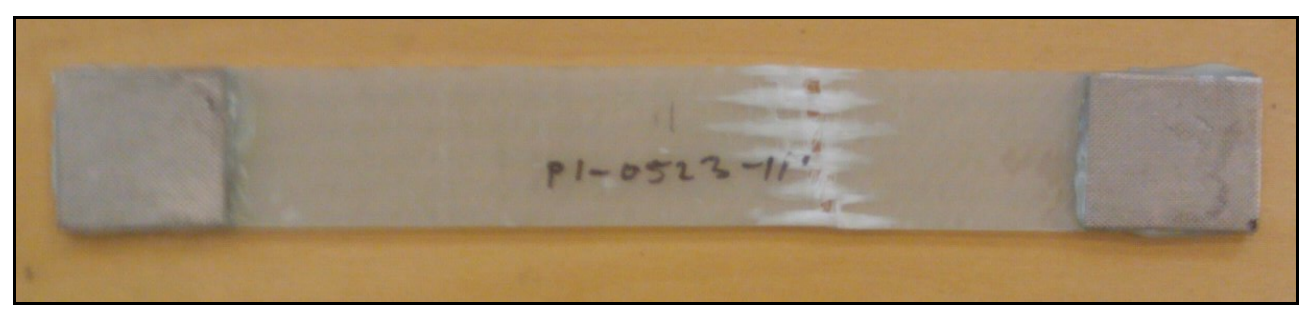




\section{Figure 61: Outcome from Manufacturing Composite Strips}

\subsubsection{Roughening the Tabs}

The tabs are the simplest part of all the manufacturing processes. These tabs were received pre-cut as show in Figure 62.

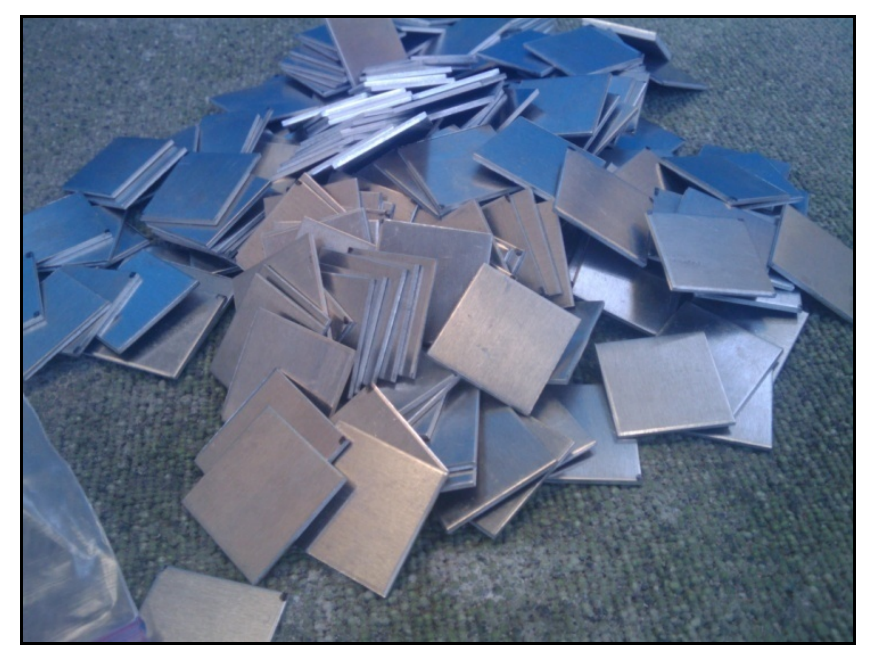

Figure 62: Pre-Cut Tabs

Each of the tabs is then roughened up using a rotary tool with a grinding bit. The purpose to roughen the surface of the tabs to provide more area for better adhesion between the test specimens and the supporting tabs. This can be seen in Figure 63.

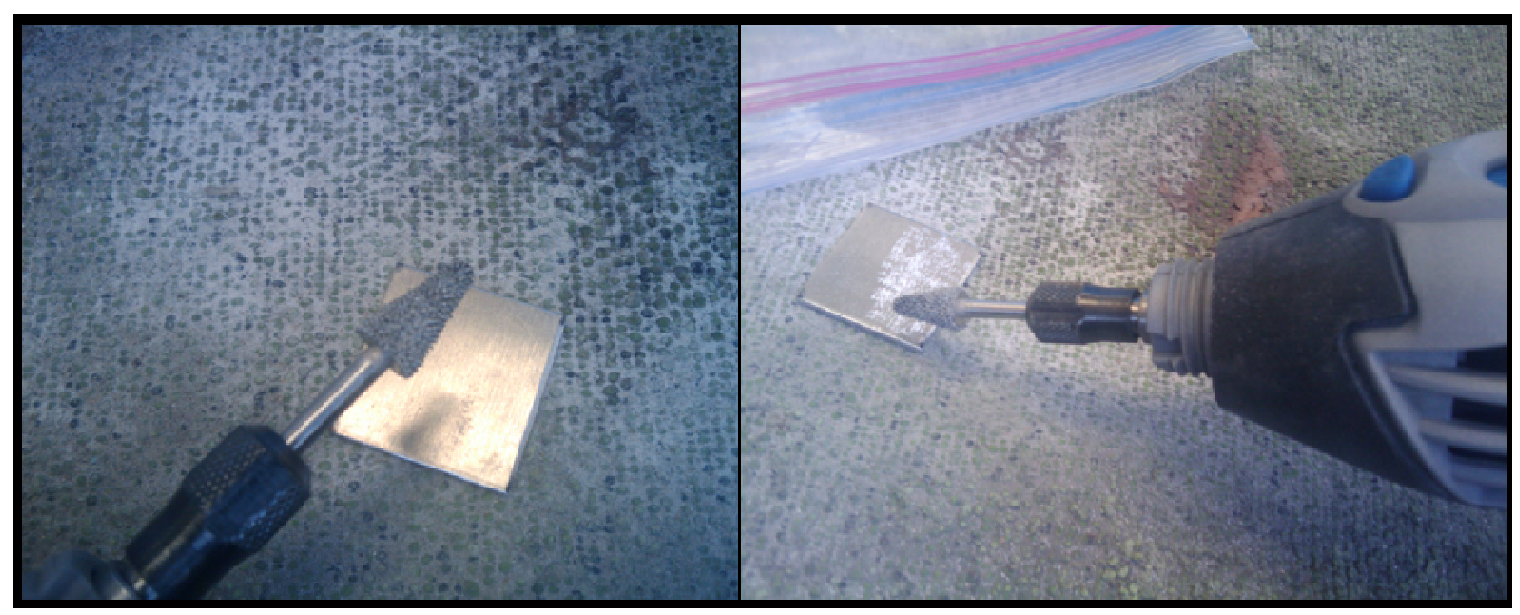

Figure 63: Roughening the Tabs

It is important to roughen the surface enough to have the best adhesion. What is done to these tabs is the roughening strokes are completed in two different directions. From the figure 
below, the image shows the transition from a plain tab to a one direction grinding to a two direction grinding. The two grinding provides the most surface roughness per rotary tool with the grinding bit.

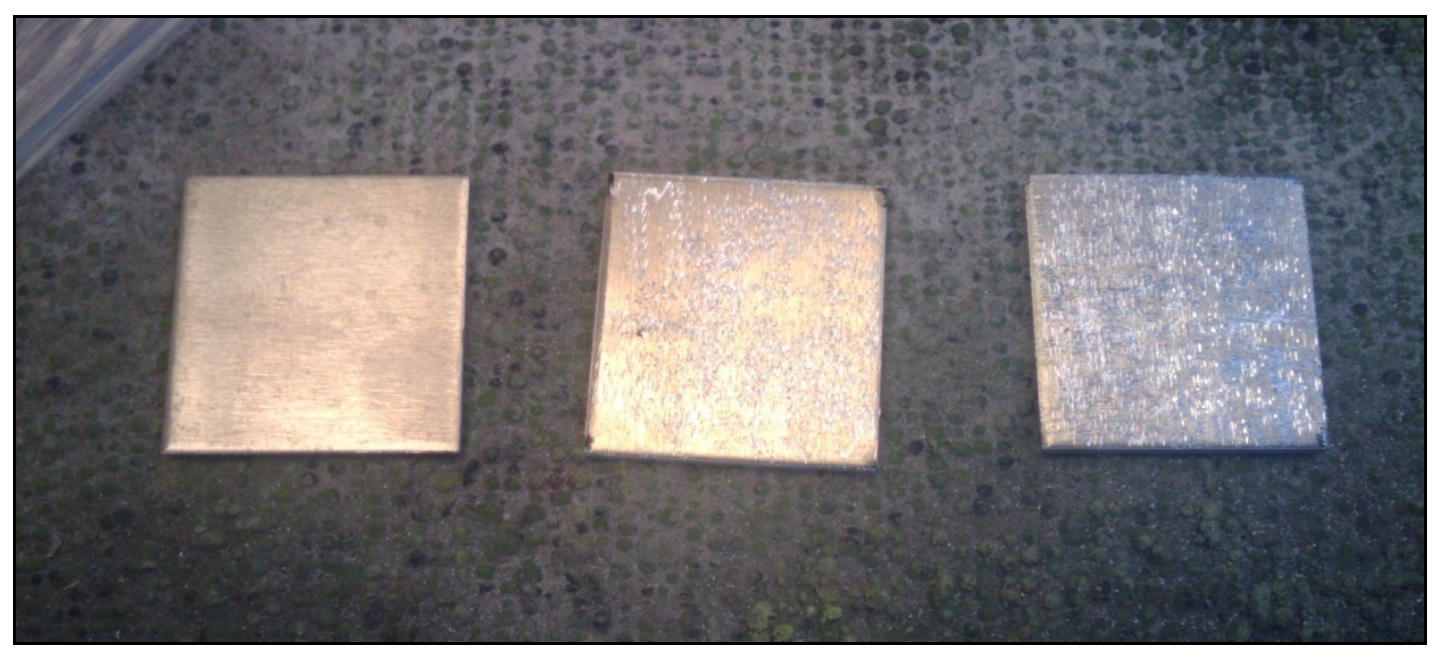

Figure 64: Tab Transitions

\subsubsection{Adding Strain Gages}

The strain gages are added to several of the test specimen to obtain the strain in the horizontal direction. To do so, an M-Bond 200 Adhesive Kit is used as the adhesive between the strain gages and the surface of the test specimen. Figure 65 shows the kit with its resin and hardener.

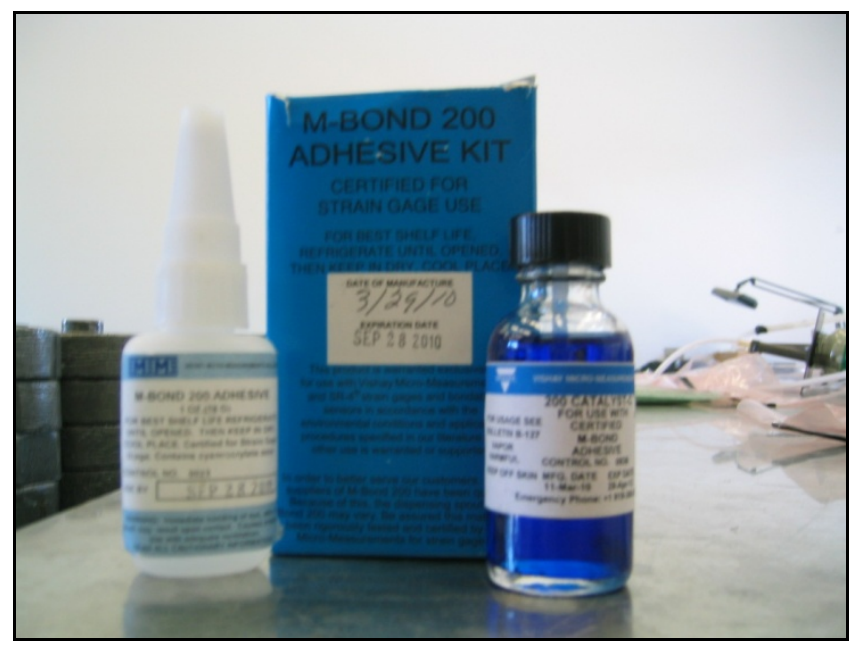

Figure 65: M-Bond 200 Adhesive Kit 
To start off, the surface of the composite strip needs to be clean in order to have the best adhesion for the strain gage to the surface. A swab with a few drops of cleanser that comes in the kit is used. The swab is stroked in one direction only to remove contaminates and not to spread them. The swabbing can be seen in Figure 66.

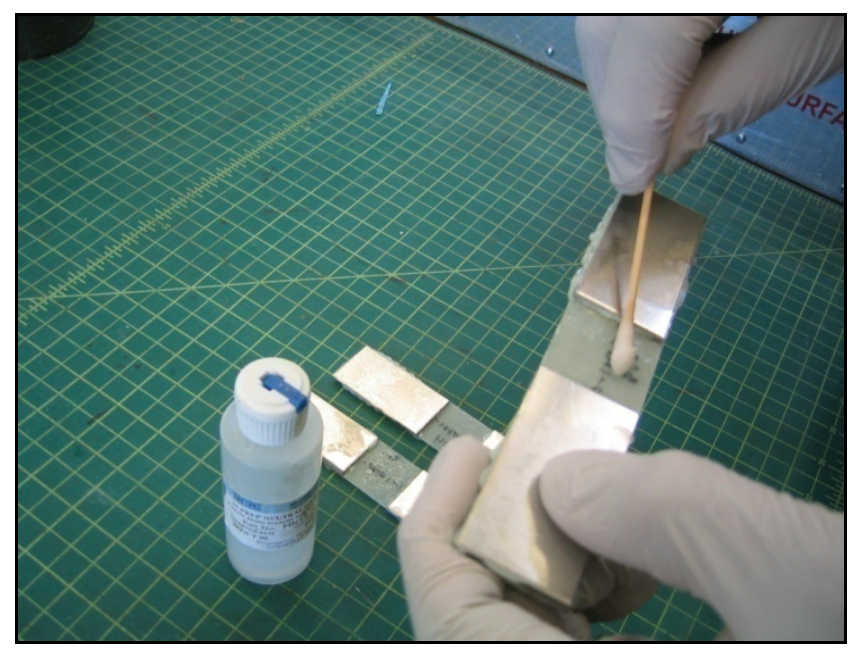

Figure 66: Cleaning the Surface

Using tongs for the small strain gage, the gage is placed on the surface of the composite strip where the gage will later be permanently positioned. With scotch tape, it is placed over the gage and lead strip as a means to hold the strain gage in the correct position and remove it from the surface. This is to maintain the correct position when it is placed back with the epoxy. This can be seen in Figure 67. 


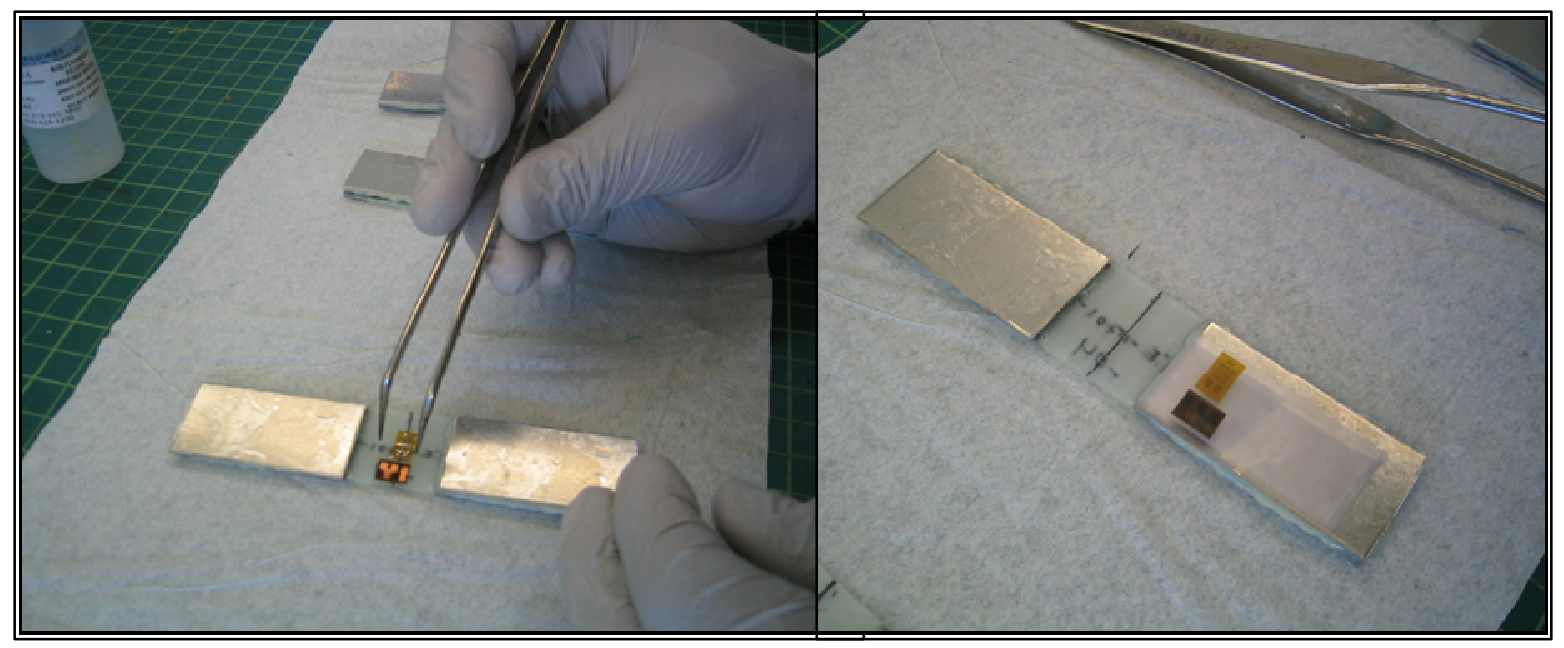

Figure 67: Strain Gage Placement and Place Holder

The resin from the kit is then applied to the surface of the strain gage and let dry for a few seconds. Note that the resin is inactive until it reacts with the hardener catalyst. The application of the resin can be seen in Figure 68.

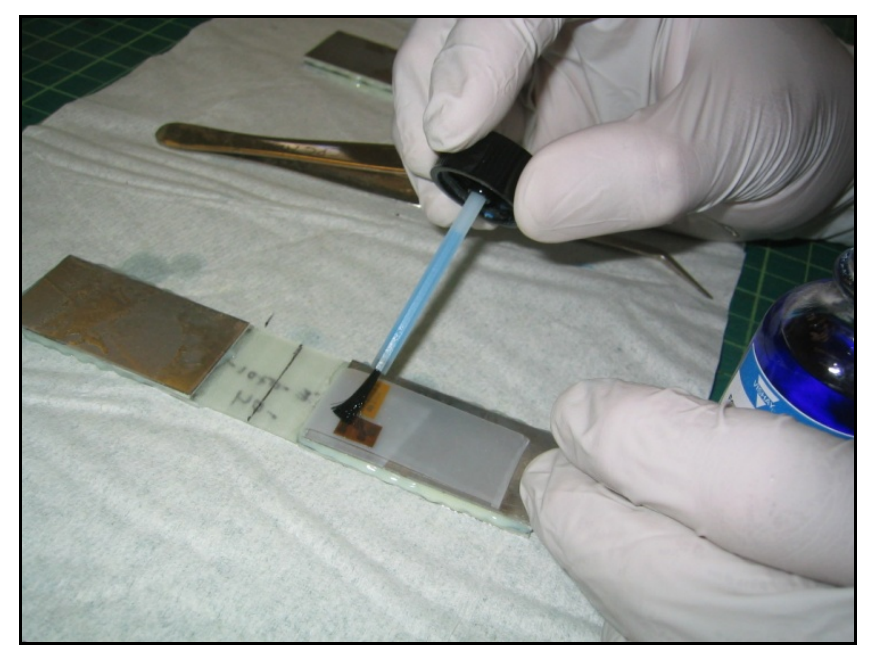

Figure 68: Applying Resin to Strain Gage

At this point, the hardener is then applied to the surface of the composite strip. The hardener dries up very quickly therefore the pre-applied resin on strain gage must make contact with the hardener as soon as possible. Pressure is then applied over the work area for approximately 2 minutes so the epoxy will cure. This can be seen in Figure 69 . 


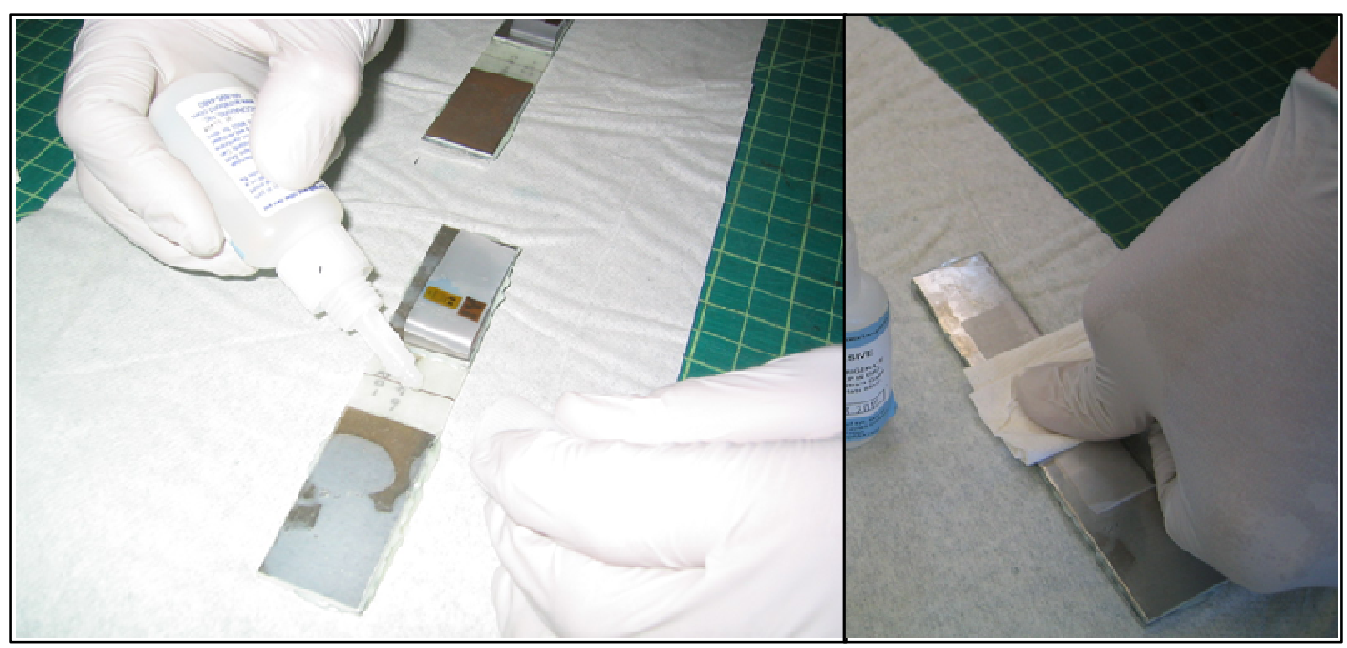

Figure 69: Applying Hardener and Pressure

The last thing on the list is to solder leads to the strain gage and verify the resistance is still with the specified requirements. An example of a completed strain gage system is displayed in Figure 70.

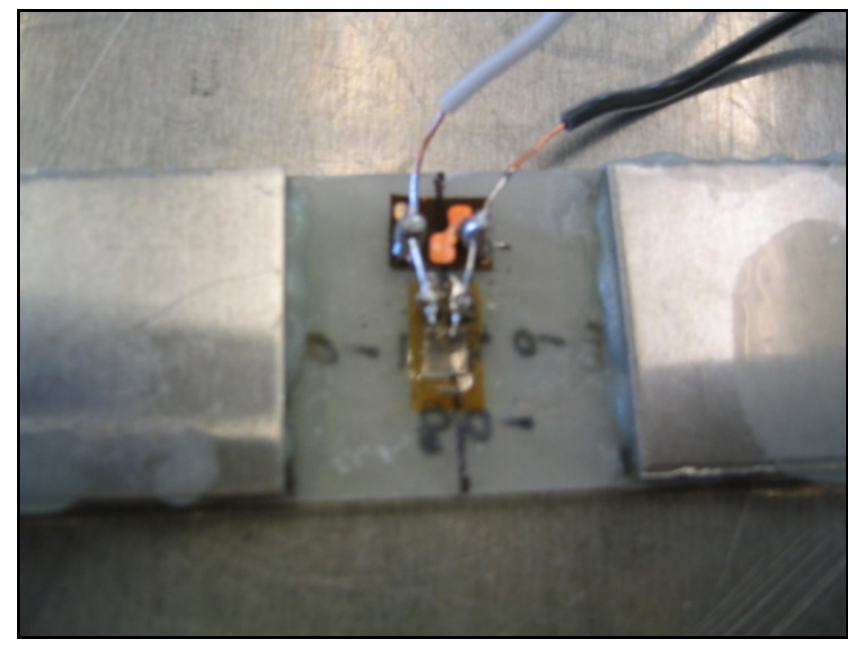

Figure 70: Completed Strain Gage System

\subsubsection{Composite Sandwich Structure Preparation}

To start the preparation of the composite sandwich structure from bare material to a fullon solid structure, the following materials are used for this project: 
Table 25: Components Required for Composite Sandwich Structure

\begin{tabular}{|l|l|l|}
\hline Type & Vendor & Model \\
\hline Fiberglass & Jamestown Distributor & FIB-947 18 oz Woven Roving \\
\hline Non-Porous Material & - & Obtained Anywhere \\
\hline Oak Wood & - & Local Lumberyard \\
\hline Flow Medium & AirTech Online & Greenflow 75 \\
\hline Peel Ply Release Cloth & AirTech Online & Econolease \\
\hline Tee Fittings & Sidewinders Computer & White Polypropylene T 1/4” ID \\
\hline Spiral Tubing & Cable Ties and More & SPW-250SP \\
\hline Tubing & Plant Lighting Hydroponic & 1/4” ID Clear Vinyl Tubing \\
\hline Sealant & The Composites Store, Inc. & $\begin{array}{l}\text { V197 Economy Vacuum Bag } \\
\text { Sealant }\end{array}$ \\
\hline Cotton Breather & AirTech Online & Econoweave \\
\hline Epoxy: Resin & Jamestown Distributors & $\begin{array}{l}\text { West System 105B Epoxy } \\
\text { Resin }\end{array}$ \\
\hline Epoxy: Hardener & Jamestown Distributors & $\begin{array}{l}\text { West System 206C Slow } \\
\text { Hardener }\end{array}$ \\
\hline Vacuum Bag Film & AirTech Online & Econolon \\
\hline
\end{tabular}

The composite sandwich structure consists of a foam core, 2 wood boundary cores, 4 sheets of the fiberglass woven roving ( 2 sheets per side), and any other components such as delamination arrestment keys and non-porous material to complete the structure. The dimensions of each item that goes into the structure varies based on the required structure size. This is usually 14 inches in length by 9.75 inches in width. In order to do the VRI technique to make the structure, several other components are required. These components are the peel ply release cloth, the flow medium, vacuum bag, sealant, cotton, and all the necessary fittings. Figure 71 shows what goes into the vacuum bag. 


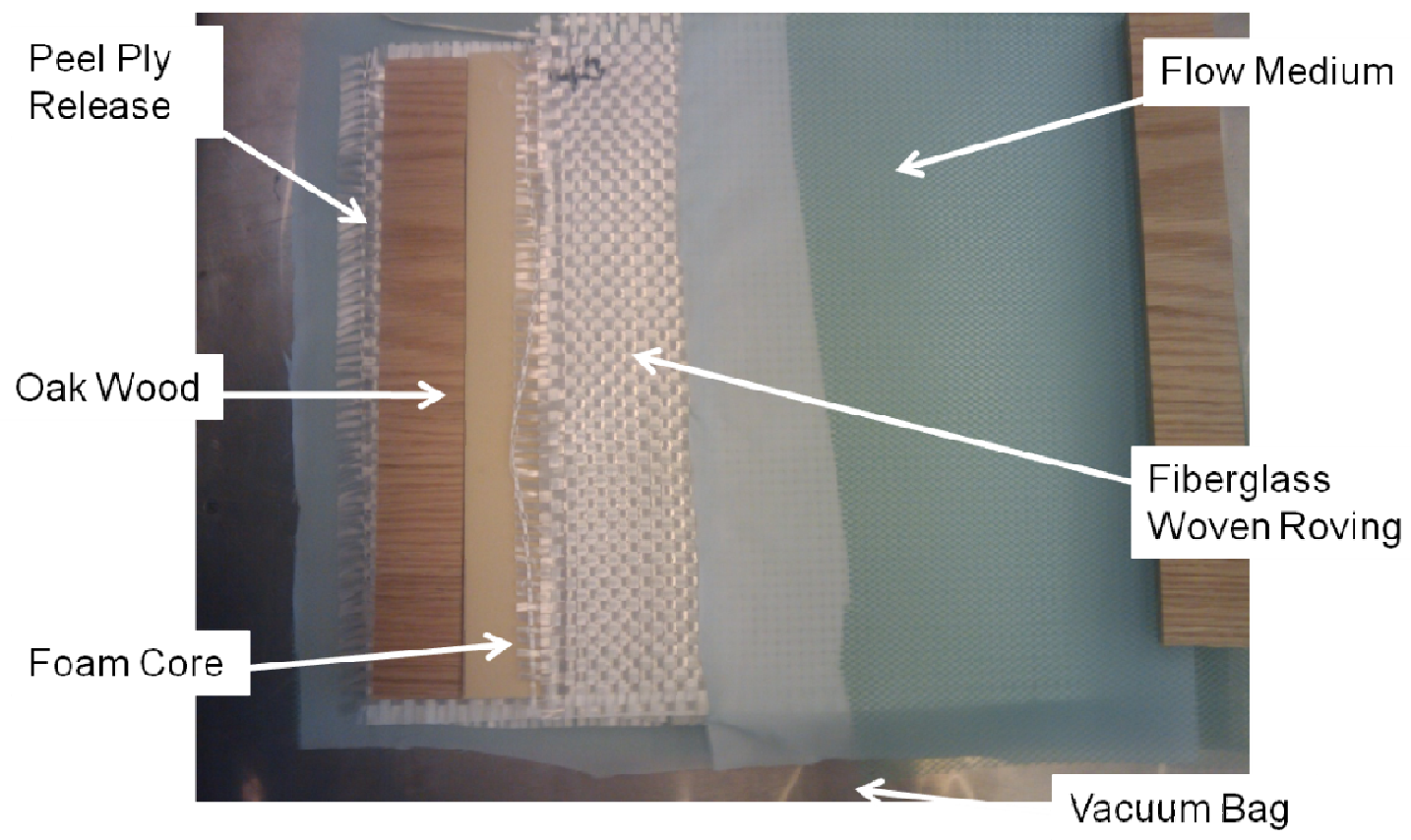

Figure 71: What Goes in Vacuum Bag

The purpose of the peel ply release cloth and the flow medium over the whole structure is to provide a means for removal of the structure after the epoxy is solid cured and for easier epoxy infusion as the epoxy is flowed from one side to the next. The vacuum bag is used to create a sealed and pressurized environment to prevent component shifts and consistency once the epoxy is infused. A diagram of how the composite sandwich structure is laid up and placed in the vacuum bag can be seen in Figure 72 .

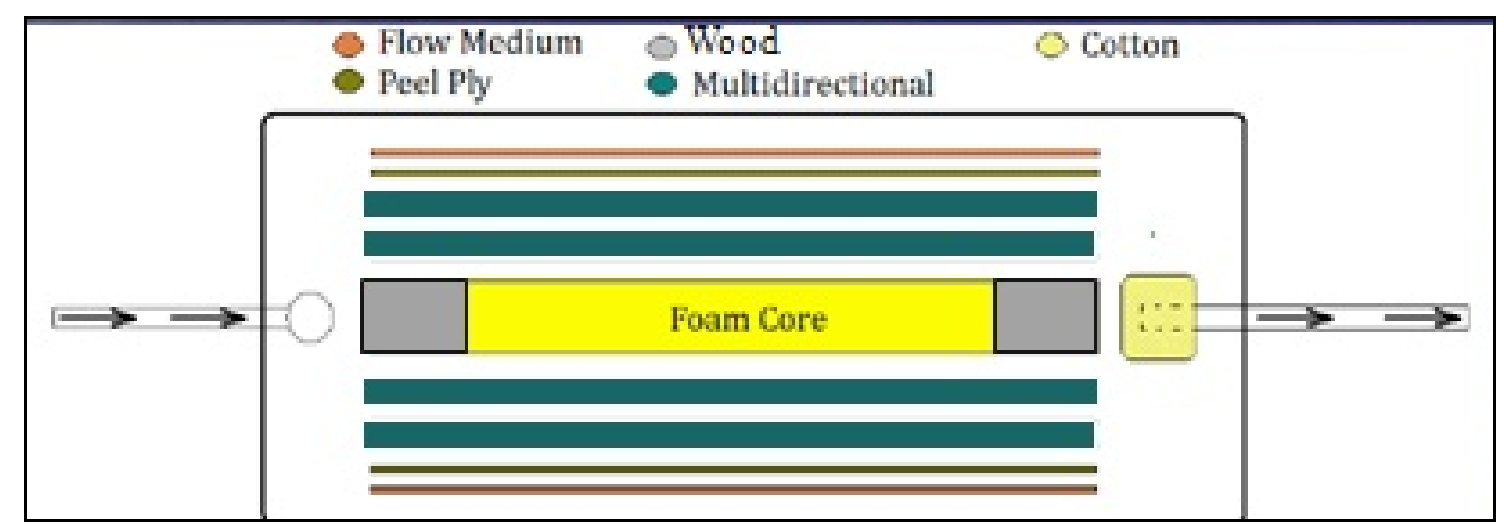

Figure 72: Composite Sandwich Structure Layup in Vacuum Bag 
The cotton padding with the vacuum bag envelope is used to absorb some of the excess epoxy as it is being pulled across the structure. As a secondary precaution, a resin trap is also used to catch all the unused epoxy in a jar. A little more about this will be discussed later.

To start off the layup, the foam core is first obtained. The foam core can be non-milled or milled which is dependent on the configuration. As discussed before, the dimensions of foam has a length of 11 inches by 9.75 inches in width by the half inch thickness. Make sure to check the quality of the foam to make sure no contaminates are on the foam surface. If cleaning is required, remove the contaminate using a shop vacuum or other cleaning materials that do not damage nor change the foam properties. Next, the wood boundary cores are obtained. The wood surfaces are roughened up a bit using 80-grit sandpaper and manually stroking the surfaces and clean off in a similar matter as the foam core. The wood boundary core is then laid next to the foam core as seen in Figure 73.

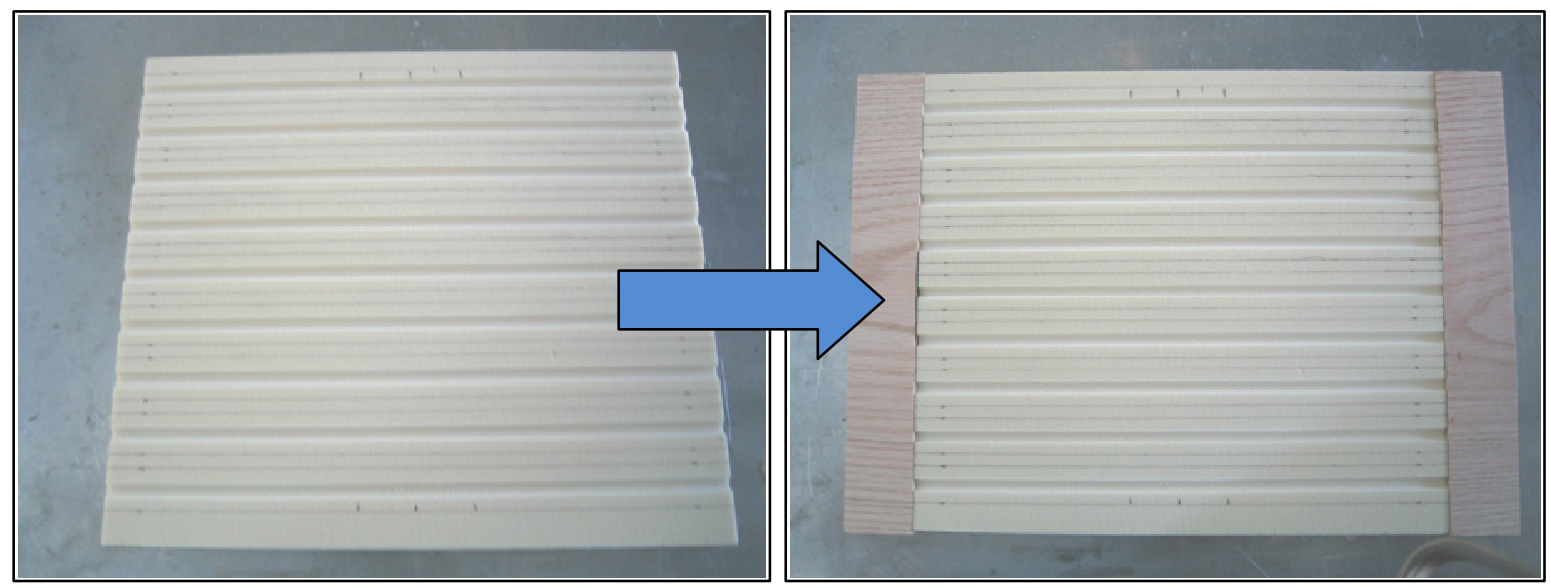

Figure 73: Foam and Wood Cores

Depending on the requirements per configuration, the components such as the non-porous material and arrestment key are placed into the placed as seen in Figure 74. The left image denoted by 'ND' is the non-delaminated test specimen with only the embedded keys. The middle image is denoted by 'SD' and has the non-porous strip material lain over the keys and placed center of the specimen. The 'SD' specimen has the skin delaminated from the key and the foam core. The right image is denoted by 'KD', which is where the skin and key is delaminated from 
the foam core in the delamination region. The keys in this configuration are placed over the nonporous strip to create the delamination when the structure is solid cured. Any of these configurations will go into the same VRI process and layup according to the configuration needs. More details regarding how the keys and non-porous will be discussed later in its subsections.

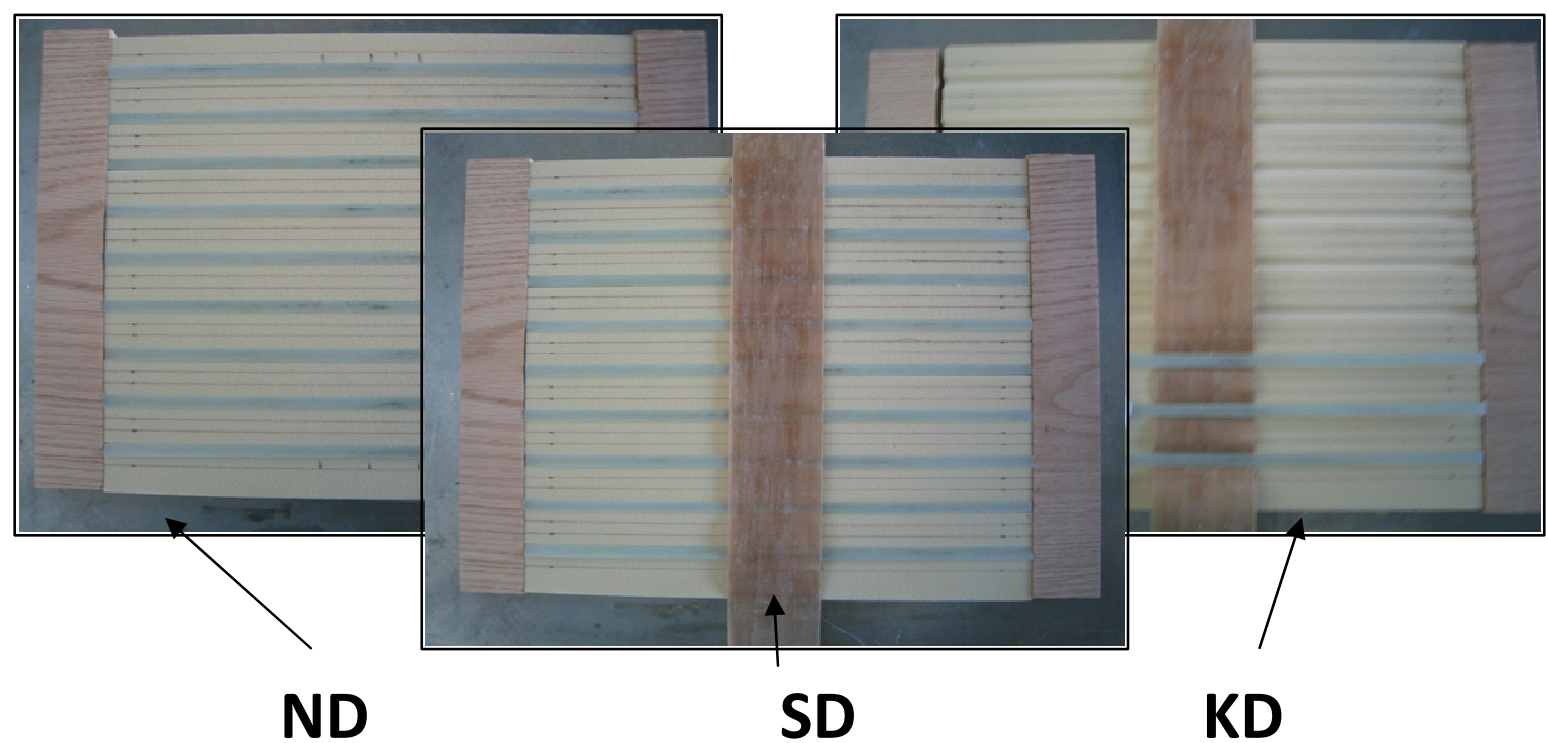

Figure 74: Different Layup Configurations

With all configuration components layup accordingly, the fiberglass sheets are placed over and under the configured structure. There are two sheets per side and they are marginally oversized to provide an inch margin on all sizes. This can be seen in Figure 75.

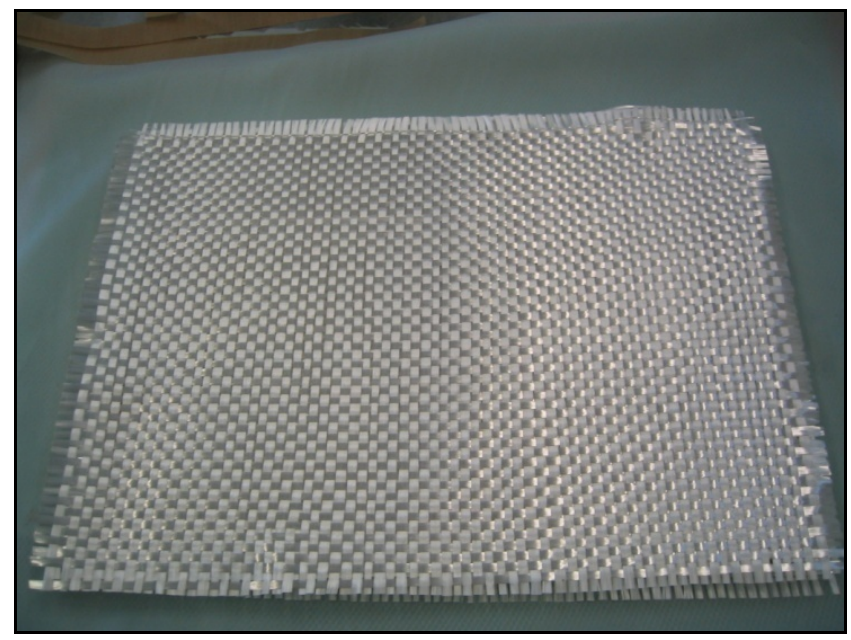

Figure 75: 2 Layers of Fiberglass on Top and Bottom 
The next steps are to obtain the peel ply release and flow medium materials. Note that the peel ply release material is laid down before the flow medium is placed. The same goes for the bottom side of this layup. From Figure 76, the images show that the peel ply release cloth and the flow medium are larger than the fiberglass sheets. The reason for the larger pieces of these materials is to cover the entire structure, allow for easier epoxy flow, and more efficient cloth and flow medium removal.

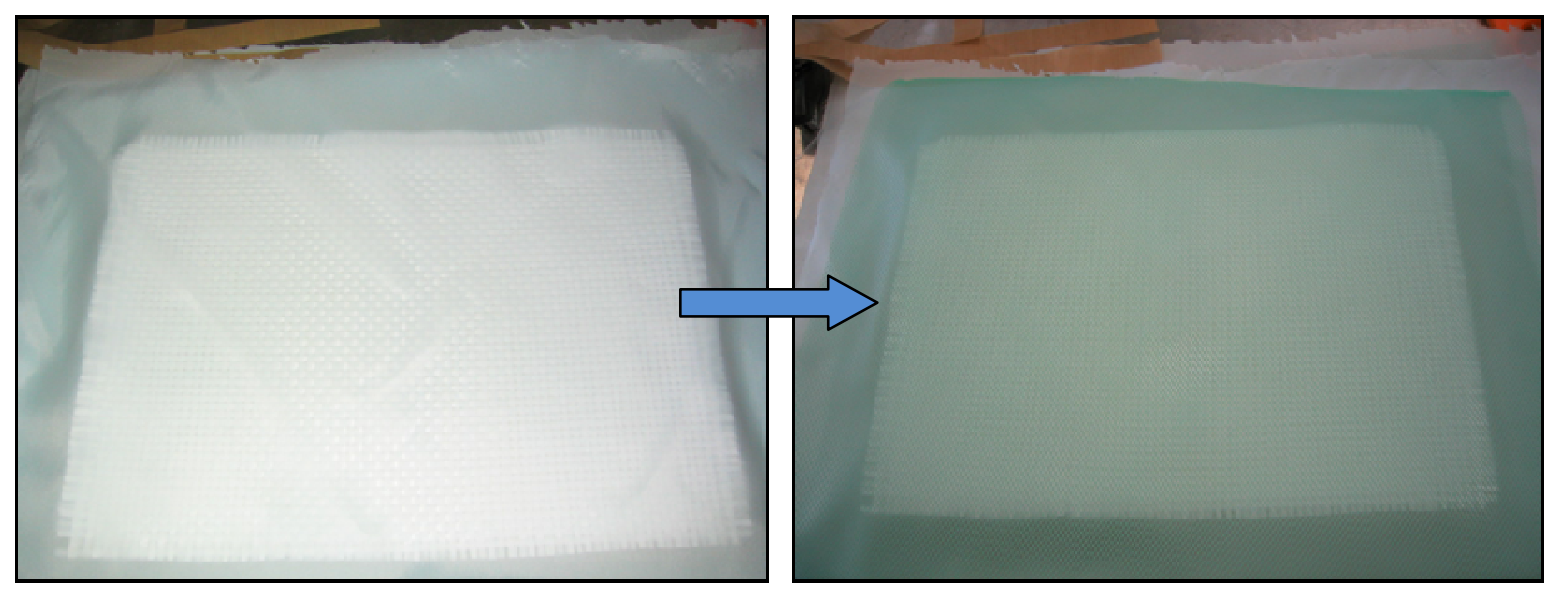

Figure 76: Peel Ply Release Cloth and Flow Medium

After all the materials are laid up on both sides of the structure, it is placed into a vacuum bag where the structure is sealed and vacuumed. The selant is placed along the rims of the structure with an approximately 2 to 3 -inch margin on all sides. This can be seen in Figure 77. Also, the tee fitting is jointed to the tubing and wrapped with the spiral tubing as seen in the same figure. The other end of the bag has a tubing where the outflow will be. The main problem discovered using this process is that if it is not sealed properly, there will be air contaminates in the structure. To avoid that, the areas surrounding the tubings need to be sealed with sealant and pressed firmly on the lining seal. To avoid contaminates on the seal, the non-stick paper on the seal is not removed until it is time for the bag to fully cover the structure. 


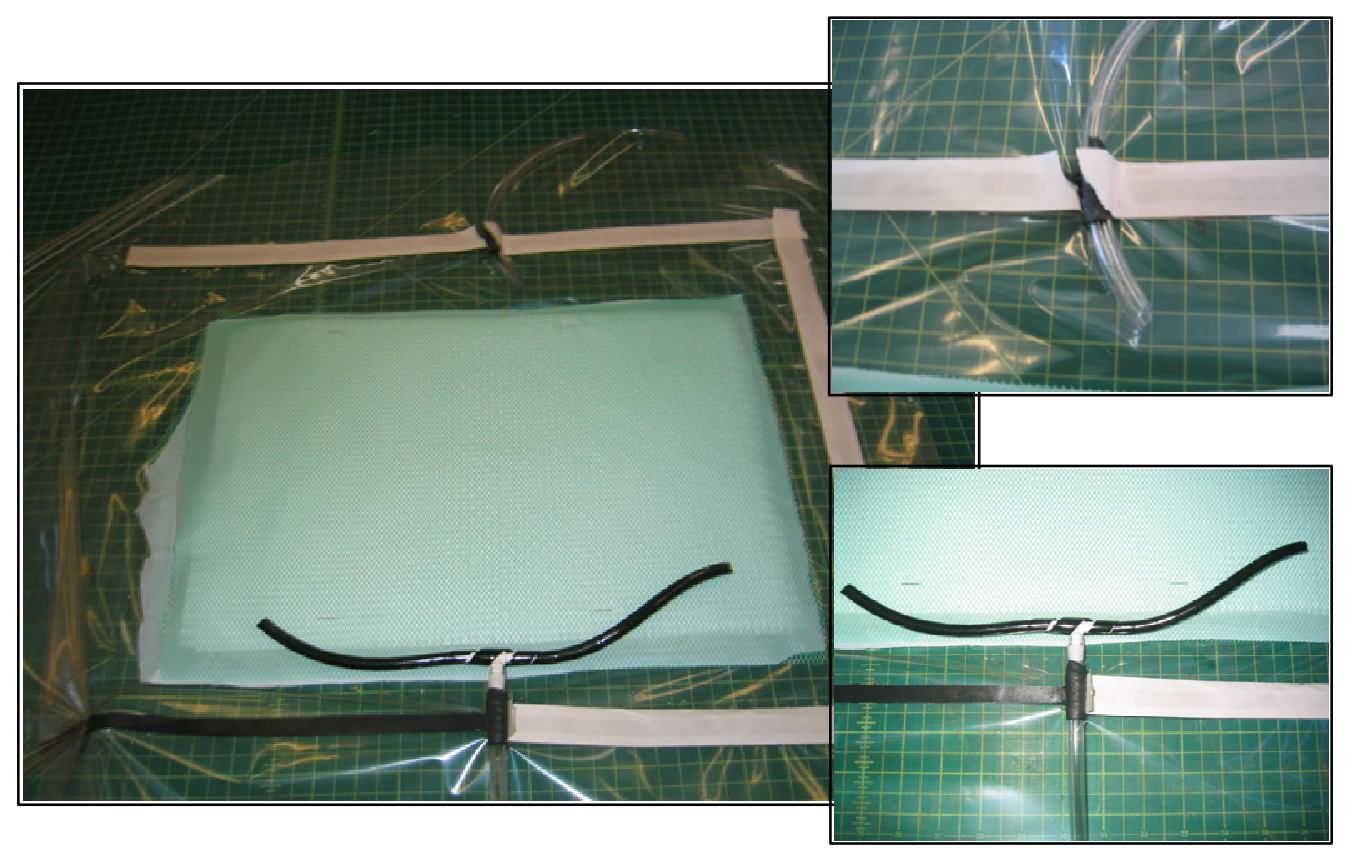

Figure 77: Structure in the Vacuum Bag

The cotton breather is placed at the outflow tubing within the bag envelope and not on the seal. Make sure that the cotton breather is covering the tubing and touching the flow medium as shown in Figure 78. The purpose for this allows for the outflow tubing to not be closed off by the bad material when the vacuum is on and to allow for the epoxy to travel more smoothly in the secondary trap. Next, the bag film is wrapped around the structure and sealed at the seal lining. At this time, visual inspection is done along the seal lining to remove any possibilities of leaks. The common method for removing leaks on the seal is to remove the air channels by sealing off the channels.

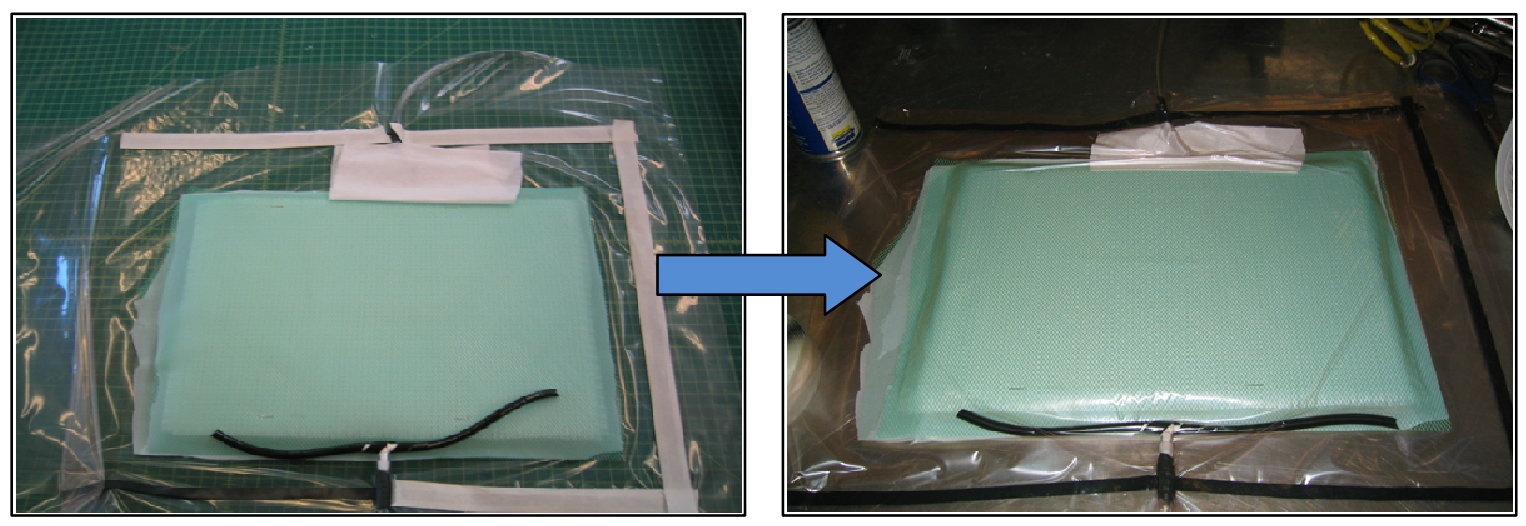

Figure 78: Cotton Breather to Sealing the Bag 
After all the seal lining is checked, the vacuum is turned on and the inlet tubing is manually clamped as a final check is done where the structure is pressurized as seen in Figure 79. Visible and auditory inspection of the seal lining and all connection points are checked for leaks. Also from the figure, the resin trap is connected to the vacuum bag and the vacuum itself. This removes any chances that the excess epoxy will go into the vacuum system.

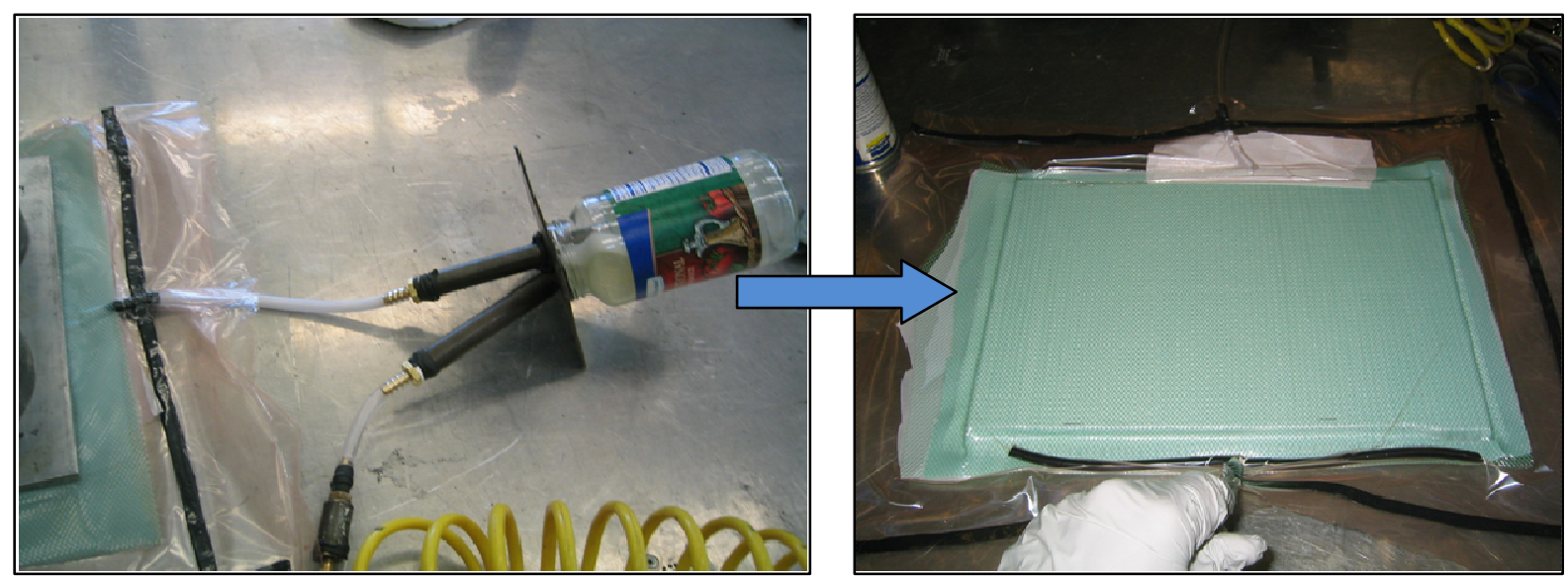

Figure 79: The Resin Trap and Final Inspection

Once the correct amount of epoxy is obtained, the epoxy is fed through the inlet tubing and flows across the structure where the epoxy wets the fiberglass skin and bonds to the cores. The volume of the epoxy is obtained from a ratio of the weighted structure. Once the epoxy completely saturates the structure, a clamp is used to stop the inlet flow. The epoxy within the structure at this point will continue to distribute itself uniformly across the composite sandwich structure because of the pressurized environment the structure is in. At this point, the resin will start to solid cure and is left to slow cure for a good 24 hours or so before it is removed from the vacuum bag. Figure 80 shows how the clamp is placed on the inlet flow and how the structure looks after the epoxy is infused. 


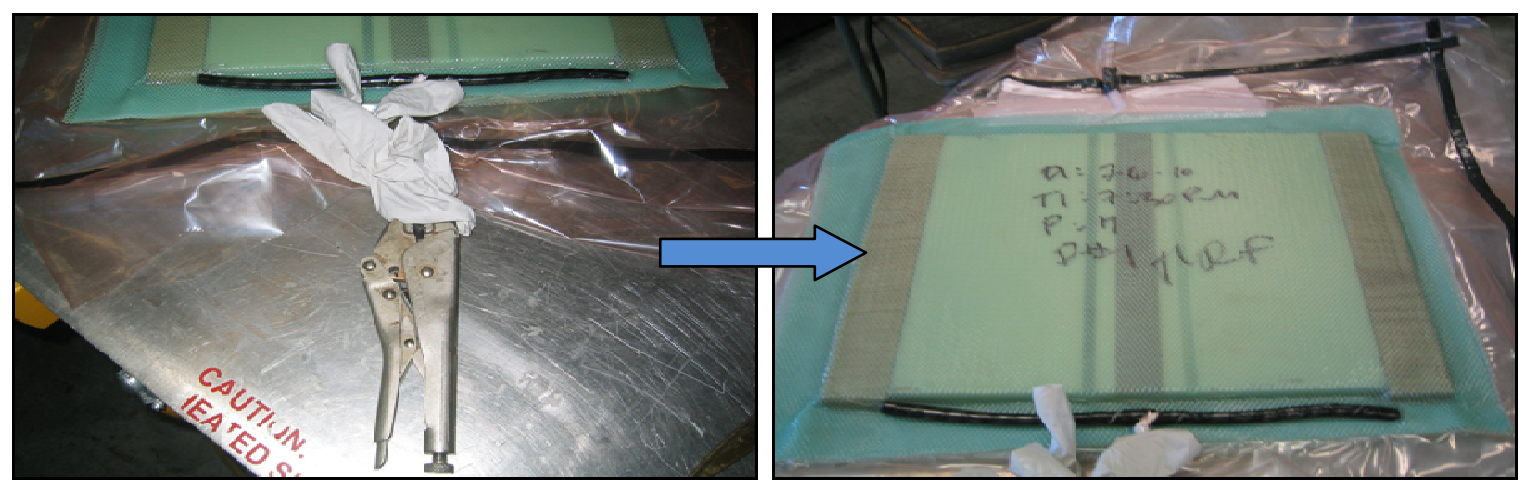

Figure 80: Clamping of Inlet Flow and Infused Structure

\subsubsection{Adding Delamination Region}

During the configuration step of the structure before it becomes vacuumed and goes

through the VRI process, some of the configurations would be introduced with a non-porous strip material waxed from top to bottom. An average of 2 to 3 strips are placed on top of one another to make sure an initial delamination region is created in the composite sandwich structure during the manufacturing process. Make sure that the wax material will not change the material characteristics of the adjacent components. An example of the waxed non-porous can be seen in Figure 81 .

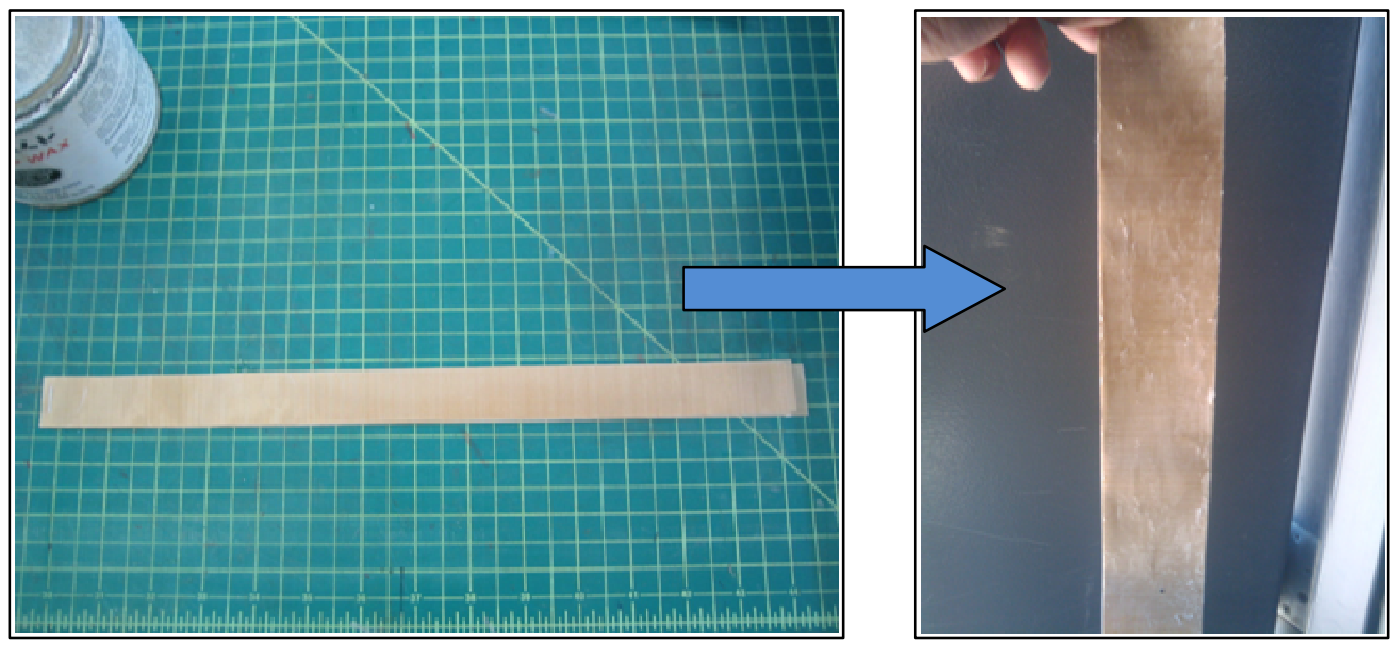

Figure 81: Waxed Non-Porous Strips 
The waxed non-porous strips are then placed in the center of the composite sandwich structure usually in between the foam core and fiberglass skin. The strips on the foam core can be seen in Figure 82.

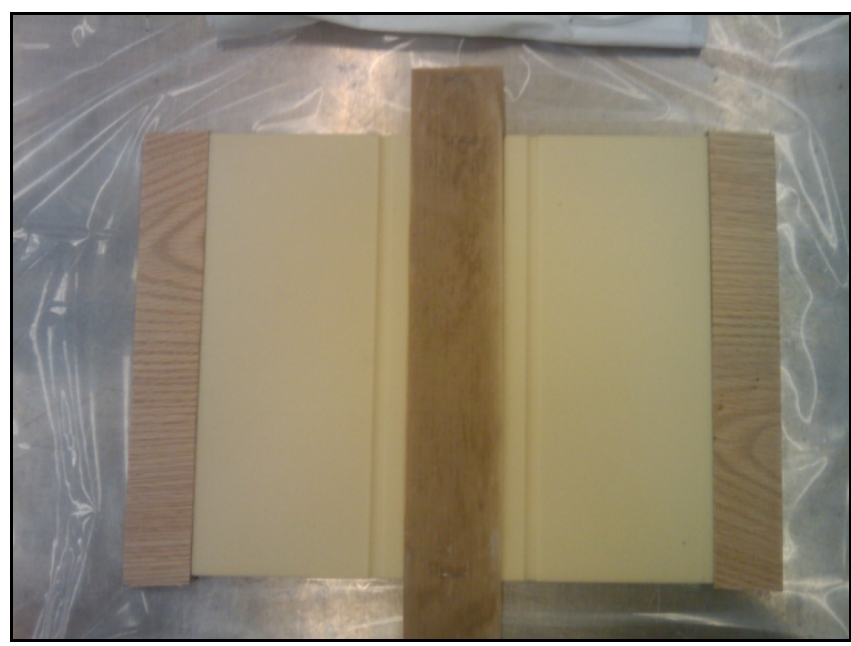

Figure 82: Waxed Non-Porous Strips on Foam Core

\subsubsection{Embedding Delamination Arrestment Key}

The embedding of the delamination arrestment keys start off with the milled out foam core. The milled foam core is dependent on the composite sandwich configuration chosen during the layup. The length of the arrestment keys are also dependent on the configuration and match the length of the grooves in the foam core. A small amount of epoxy is coated on the key before it is placed into the grooves to provide better bonding to the foam and the composite skin. Note that with the mixed epoxy on the keys, there is a limited time period before the epoxy begins to crystallize thus this requires integrating the structure and following through with the VRI process in a timely manner. Figure 83 shows an example of how the arrestment keys are wetted and placed in the grooves. 


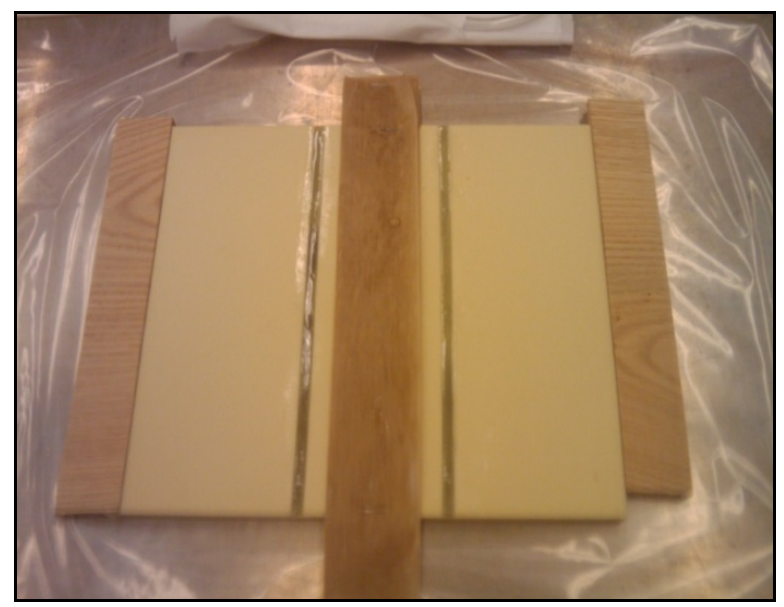

Figure 83: Key Placement in Foam Core

The 'PL' configuration which is denoted by its discontinuity in how the arrestment keys are placed requires a bit more attention. During the milling of these grooves, it is impossible for the ends of the grooves to be flat thus it is rounded per ball end mill bit. To minimize the issue, epoxy is poured into these ends as filler. The arrestment keys are coated with the epoxy and inserted in the same process. The epoxy at the ends will then harden and bridge the gaps between the ends and the keys. Detail images of the epoxy filler and the key embedment in the 'PL' configuration is shown in Figure 84.

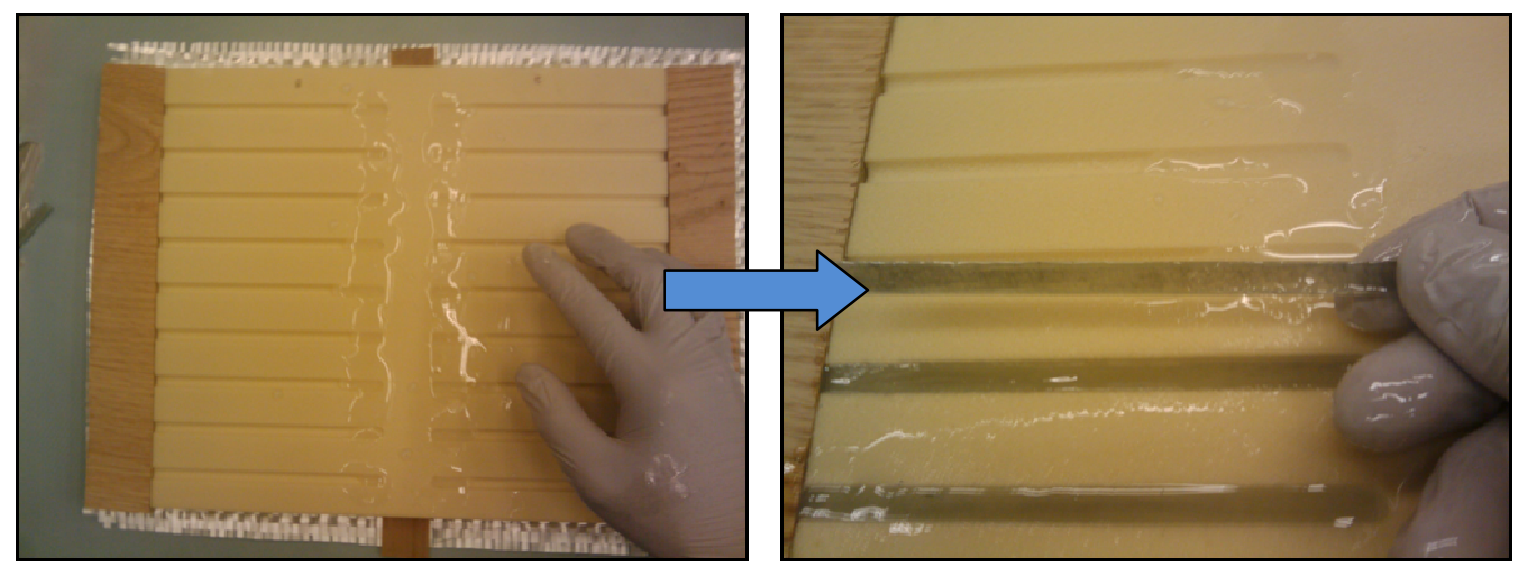

Figure 84: Epoxy Filler and Key Embedment for 'PL' Configuration

\subsubsection{Specimen Preparation}

After all composite sandwich structure is to cure for a day or so, it is then removed from the vacuumed bag, the used flow medium and peel ply cloth are discarded, and the test specimen 
is cut down to the correct dimensions using a wet tile saw. The purpose of a wet tile saw is to avoid burns and delamination during the cuts. Safety is a priority when manufacturing and preparing these test specimens. The appropriate attire and safety equipments are used at all times during the specimen preparation process. Figure 85 shows the wet tile saw used for the preparation, and the structure before it is cut down.
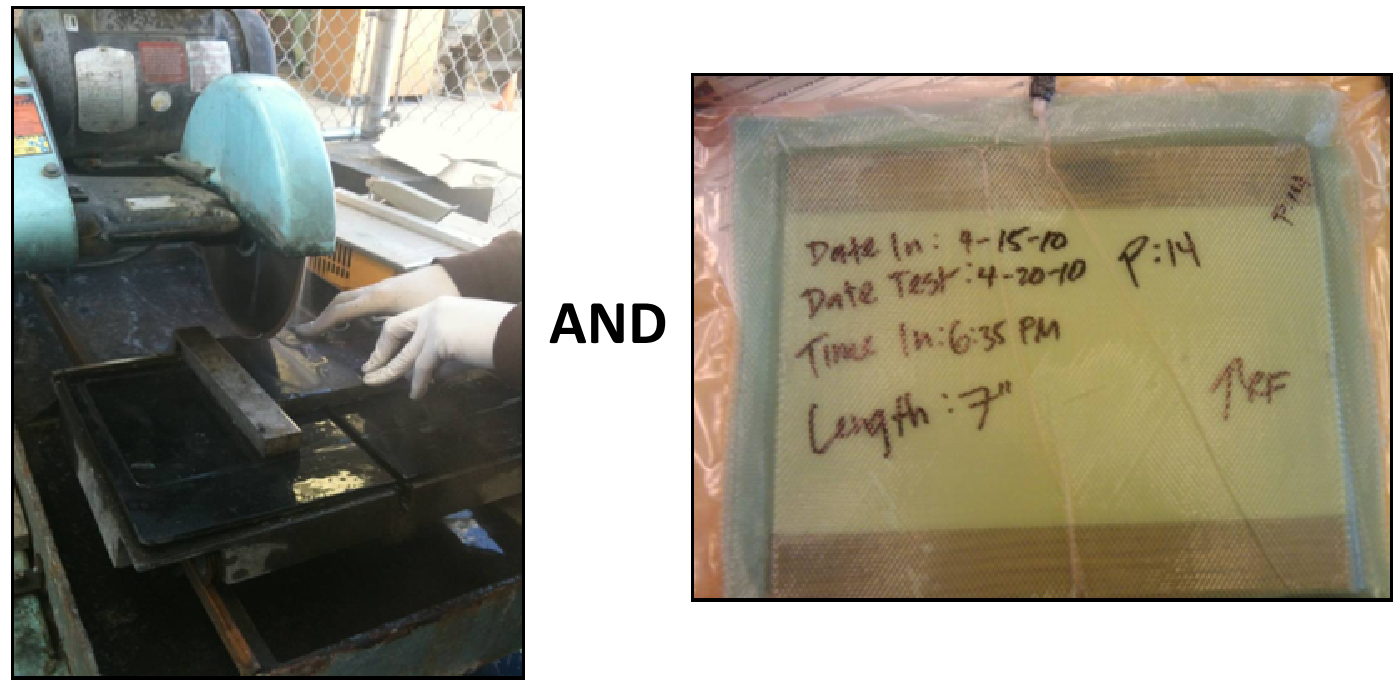

Figure 85: Wet Tile Saw and Pre-Cut Structure

After the specimens are cut down, they are measured and recorded for future processing. Lastly, the test specimens are drilled through the wood core forming the correct boundary conditions needed for buckling to occur. Any burrs at the drill site are removed. Examples of the cut down specimens and the drilled hole can be seen in Figure 86. 


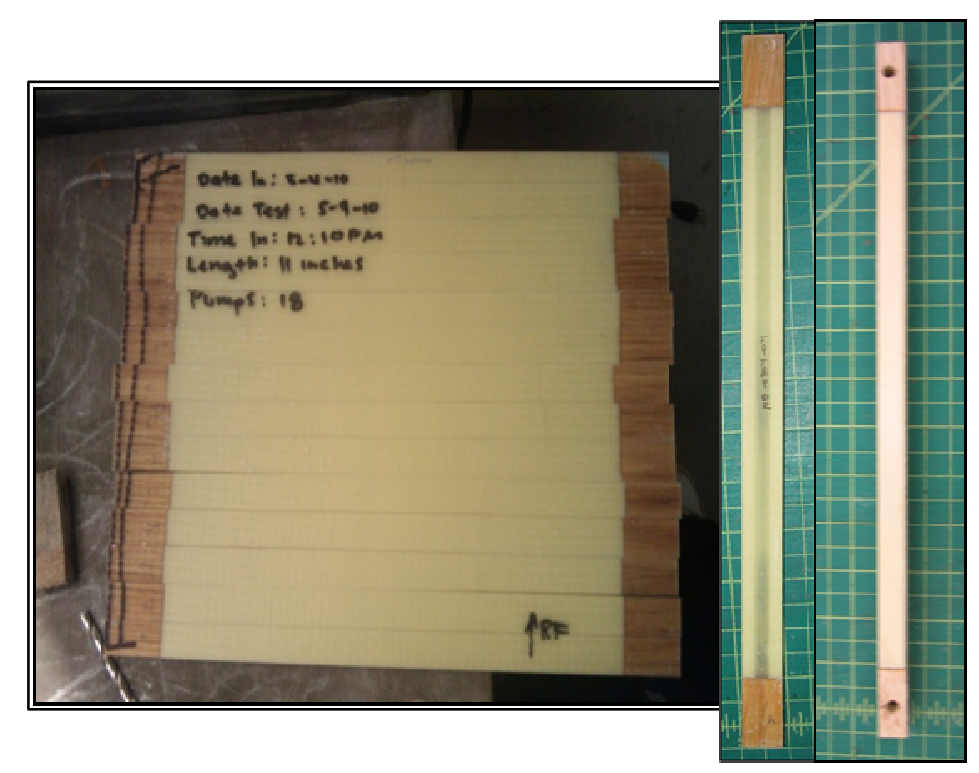

Figure 86: Cut Down Specimens and Drilled Holes

\subsection{Testing Procedures}

This section will be discussing the different procedural steps required to perform the experimental testing of the specimen to obtain its mechanical properties. This section provides a step by step procedure in how these tests are performed and how the results are recorded.

\subsubsection{Weight and Volumetric Fraction Procedure Testing for Face Sheet}

The weight and volumetric fraction testing is done on face sheets of the composite sandwich structures. The process starts off by removing the face sheet from a previously tested specimen separating the skin from the foam core. Using a belt sander, the face sheet is sanded down to remove any residual foam left over during the separation. The purpose of sanding down the structure is to remove as much error in order to accurately find the weight and volumetric fractions. The fiberglass skin is then cut down to be 0.75 inch by 1.0 inch using the wet tile saw. The size of the specimens is limited because of the dimensioned width of the composite sandwich specimen. An example of the cut down fiberglass face sheet is shown in Figure 87. 


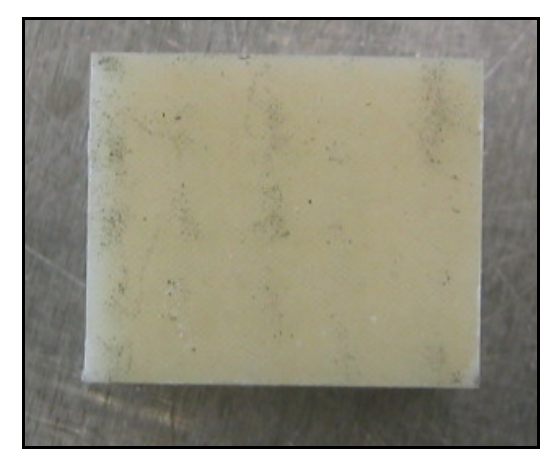

\section{Figure 87: Cut Down Fiberglass Face Sheet}

The dimensions of each test specimen are measured and recorded for future processing. The weight of each test specimen is also recorded. These test specimens are then placed into the furnace and burned at a temperature of 1100 degrees Fahrenheit. The test specimens stay in the furnace until all the epoxy is burned off and the fibers are left; this can be seen in Figure 88 .

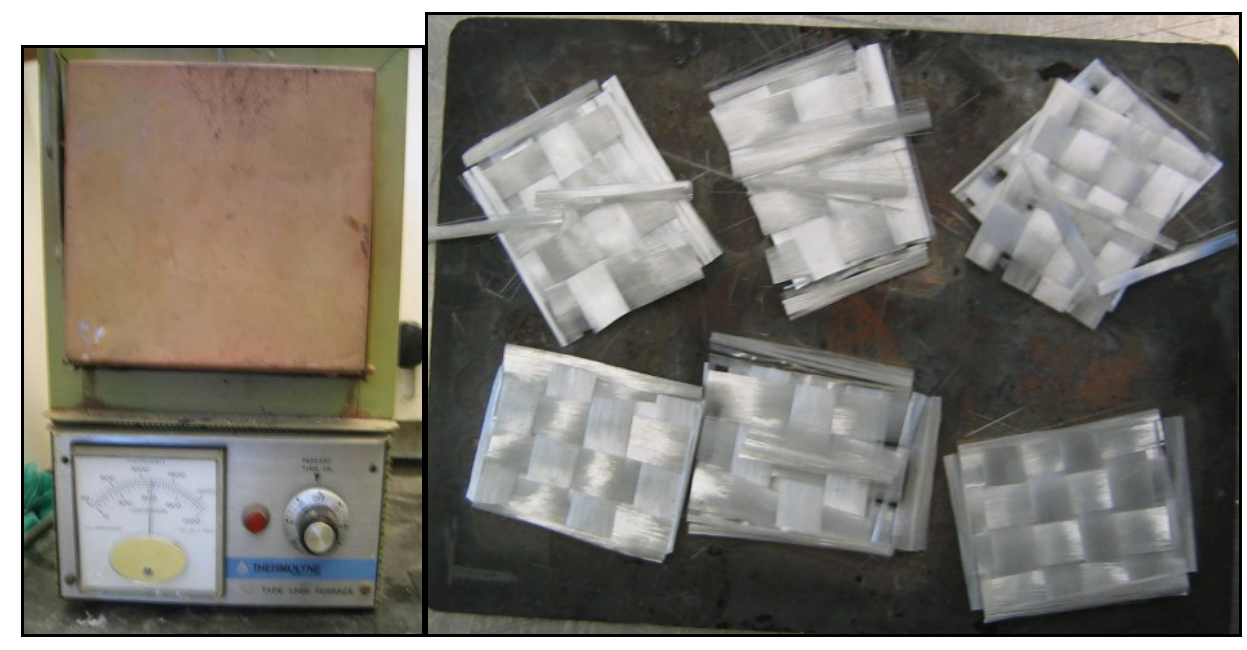

Figure 88: Volumetric Testing of Fiberglass Skin

The fibers are then weighed again for later processing for the volumetric fraction of the fiber to matrix. Each of these specimens are numbered and stored for records.

\subsubsection{Weight and Volumetric Fraction Procedure Testing for Arrestment Keys}

The weight and volumetric testing of the arrestment keys is completed the same way as the fiberglass face sheet. The only difference is the dimensions. The arrestment key is cut down to have a length of 1 inch and maintain its 0.25 inch semi-circular cross-section. The weights are also recorded in the same manner as the face sheet test specimens. 


\subsubsection{Composite Skin Testing for Material Properties}

As discussed before, the design to manufacturing is directly used from ASTM D-3039 and ASTM D-3410 standard methods with slight modifications to the specimens in order to fit the wedge grips on the Instron displacement machine at California Polytechnic State University at San Luis Obispo, California. The wedge grips are closed onto the aluminum tabs of the test specimens and loaded either in tensile or compression. The quasi-static strain rate used by the standard methods is 0.05 -inch per minute and controlled digitally by Bluehill 2 software.

With the help of Bluehill 2 software, as the displacement of the specimens begin, the software records all the loads and extension per given system interval. This is later used for processing for other material properties such as the material's moduli, stresses, and strain.

\subsubsection{Testing for Material Properties using Plate-Plate Boundary}

Similar to the compressive skin testing procedure, this test is only done in compression with the same quasi-static strain rate of 0.05 -inch per minute. The tee-shaped jigs are placed in between the wedge grips attached to the Instron displacement machine. Note that the tee-shaped jigs must be aligned and properly balanced before adding test specimens and loading it.

Prior to putting the test specimen between the jigs, the dimensions of the test specimen are measured and recorded for future processing. A test specimen is then placed in between the jigs and strapped with a strain transducer in order to get the strain in the horizontal direction and recorded using Bluehill 2 software. The software will digitally control the displacement rate and the load criteria when the specimen is loaded. All the raw data will be to be parsed with the measured dimensions of each test specimen. An example of this looks like in the Instron displacement machine can be seen in Figure 89. 


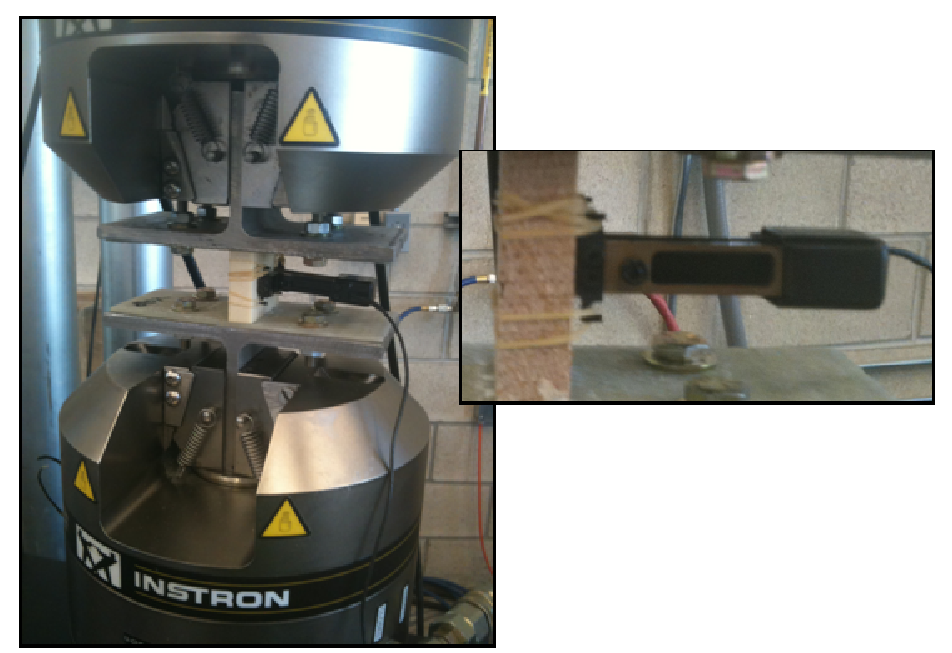

Figure 89: Testing with Plate-Plate Boundary

\subsubsection{Testing for Mechanical Behaviors and Properties using Pinned-Pinned Boundary}

Similar to the testing procedure using the plate-plate boundary, this testing procedure is also inwardly displaced. According to ASTM C-364 standard method, the quasi-static strain rate is 0.02 -inch per minute because of how the test specimen is being displaced. The setup of the test jig is mounted directly to the displacement unit and the load cell of the Instron displacement machine. Instron LVDT Displacement Gauge is placed perpendicular to the test specimen and the load vector in order to get the horizontal displacement the specimen is seeing. The test setup can be seen in Figure 90.

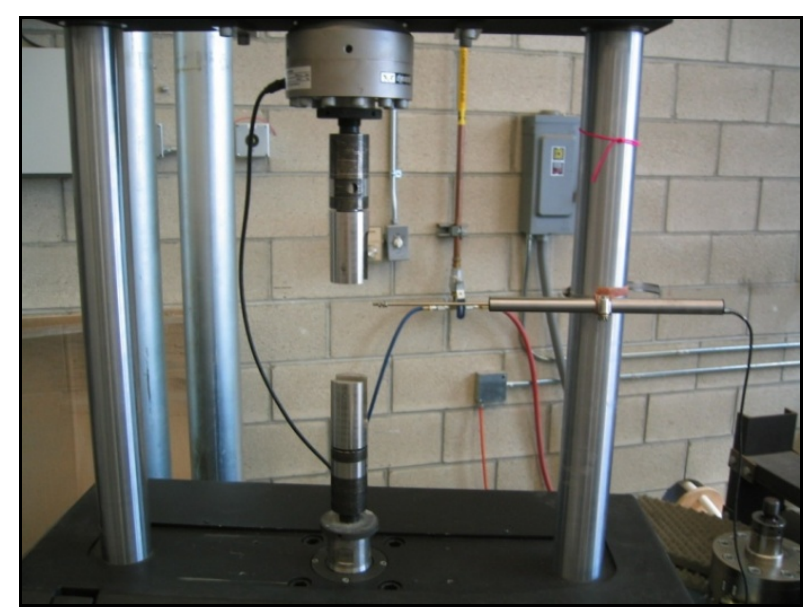

Figure 90: Pinned-Pinned Boundary Set Up 
The test specimen is placed into the pinned-pinned boundary with aircraft grade bolt and fastened to avoid any horizontal play. The specimen is only allowed to move vertically and horizontally with rotation capabilities around the hole location. The displacement gauge is lean against the test specimen at its center and measures its displacement. The specimen is loaded digitally using Bluehill 2 software and all raw data is recorded for future processing.

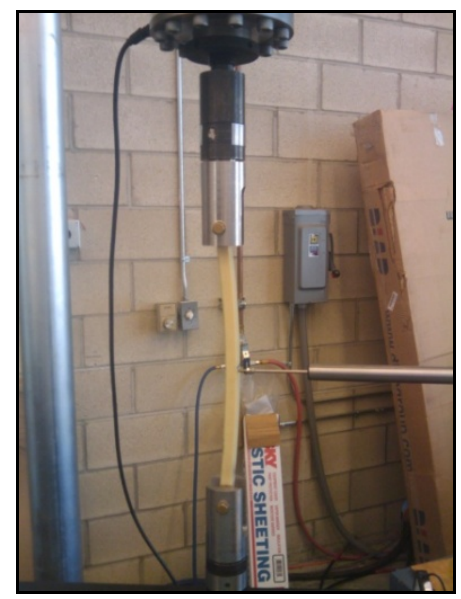

Figure 91: Testing Using Pinned-Pinned Boundary 


\section{THEORETICAL ANALYSIS}

This section of the report will be discussing the theoretical analysis of the composite sandwich structure. Analysis of the weight and volumetric fractions will be investigated to see what percentages of the fiber and matrix contents are within the composite skin and the delamination arrestment key.

\subsection{Analysis of Weight and Volumetric Fractions}

The importance of knowing what the weight fraction and volume fraction of the composite structure such as the skin and the delamination arrestment key allows for future iteration of the design. That is, the ability to correlate the strengths and weaknesses of the structure by knowing how much fiber and matrix contents is within the structure. The weight fraction is the easiest to find because it only requires the measured weights of the specimen with and without epoxy infused. This can be done during the layup process where each part is measured or during an experimental burn test to remove the epoxy and measured.

The weight fraction can be defined as $\mathrm{W}$ and the subscripts are dependent on content that is being looked at. The subscript, tot, is the total of the whole structure, F, is the fiber, and M, is the matrix of the composite structure. The weight of each component can be defined as W. The weight fraction is a ratio of the component to the whole structure. The equations that summed up the components and its weight fraction can be found the set of equations in Equation 1.

\section{Equation 1: Weight Fraction and Weight of Composite Structure}

$$
\begin{aligned}
& w_{t o t}=w_{f}+w_{m} \\
& w_{f}=\frac{w_{f}}{w_{t o t}} \\
& w_{m}=\frac{w_{m}}{w_{t o t}}
\end{aligned}
$$

The volume fraction is a bit more difficult to figure out because it requires manipulation of several equations and theoretically calculated. The volumetric fractions for the fiber and matrix 
content require density of each component which is used to correlate the component weight to find the component volume and volume fractions. Similar to component weight and weight fraction analysis equations in Equation 1, the volumes and volume fractions of each component can be seen in Equation 2.

\section{Equation 2: Volumetric Fraction and Volume of Composite Structure}

$v_{t o t}=v_{f}+v_{m}$

$V_{f}=\frac{v_{f}}{v_{t o t}}$

$V_{m}=\frac{v_{m}}{v_{\text {tot }}}$

The volume fraction is defined as $\mathrm{V}$ and has the same subscripts as the weight fraction equations. The volume is denoted by $\mathrm{V}$ and it is used to define the volume of each component material.

The relationship between the volumetric fraction and weight fraction is dependent on the density of the material P. That is, the density of the fiber and matrix need to be known in order to determine the volumes and volumetric fractions. The proportional relationship of the weight fraction to the volumetric fraction for the fiber and matrix materials can be seen in Equation 3.

\section{Equation 3: Relationship between Weight Fractions and Volumetric Fractions}

$$
\begin{aligned}
& V_{f}=\frac{\rho_{\text {tot }}}{\rho_{f}} W_{f} \\
& V_{m}=\frac{\rho_{\text {tot }}}{\rho_{m}} W_{m}
\end{aligned}
$$

Similarly, if the density of the structure is unknown, the volumetric fractions can be determine correlated with the component weight fractions only. This can be done with the equation set in Equation 4.

Equation 4: Relationship between Component Weight Fractions and Volumetric Fractions

$$
\begin{aligned}
& V_{f}=\frac{\rho_{m} W_{f}}{\rho_{f} W_{m}+\rho_{m} W_{f}} \\
& V_{m}=1-V_{f}
\end{aligned}
$$


With the volumetric fractions, it can be used to determine the Elastic Modulus, E, of the composite structure in its longitudinal, denoted by subscript long, and lateral axes, denoted by subscript lat. The equations to determine the Elastic Modulus of the composite structure can be seen in Equation 5. The Elastic Modulus in the longitudinal and lateral directions is dependent of the component material modulus, the Poisson's ratio of the epoxy material, and the volumetric fractions.

\section{Equation 5: Elastic Modulus using Volumetric Fractions}

$$
\begin{aligned}
& E_{\text {long }}=V_{f} E_{f}+V_{m} E_{m} \\
& E_{\text {lat }}=\frac{E_{f} E_{m}^{s}}{V_{f} E_{m}^{s}+V_{m} E_{f}\left(1-v_{m}^{z}\right)} \text { where } E_{m}^{s}=\frac{E_{m}}{\left(1-2 v_{m}^{z}\right)}
\end{aligned}
$$

Finding the Elastic Modulus of the composite structure is one of the variables that can be determined using the volumetric fractions. There are other uses for the volumetric fractions such as finding the ultimate stresses and strength pertaining to the composite structure. 


\section{EXPERIMENTAL RESULTS AND DISCUSSIONS}

\subsection{Configuration of Composite Sandwich Structures}

The embedment of the arrestment key into the composite sandwich structure was investigated. This investigation consisted of how delamination arrestment key was placed, varying the delamination arrestment key length based on the specific configuration, and where the delamination sizes varied per inch increment. This includes looking into the controlled configurations to see the similarities and differences between configurations. All of these groups have approximately 9 test specimens or more. All of the studied cases are listed below:

- Control Groups

○ Control Group: No Delamination and No Embedded Arrestment Key

○ Control Group: Initial Delamination with No Embedded Arrestment Key

○ Control Group: No Delamination with Embedded Arrestment Key

- Experimental Groups

- Experimental Group: Continuous Key in Vertical Direction with Skin Delamination

○ Experimental Group: Continuous Key in Vertical Direction with Key Delamination

○ Experimental Group: Continuous Key in Horizontal Direction with Skin Delamination Configuration

○ Experimental Group: Discontinuous Key in Vertical Direction with Skin Delamination

Control Group: No Delamination and No Embedded Arrestment Key - This control group is free from all experimental variables such as delamination or an embedded key within the composite sandwich structure. This section of the experimental results comes from the already 
tested 11-inch specimens when determining the test specimen length that buckled. This will later be used to compare the experimental groups to see the similarities and differences between them.

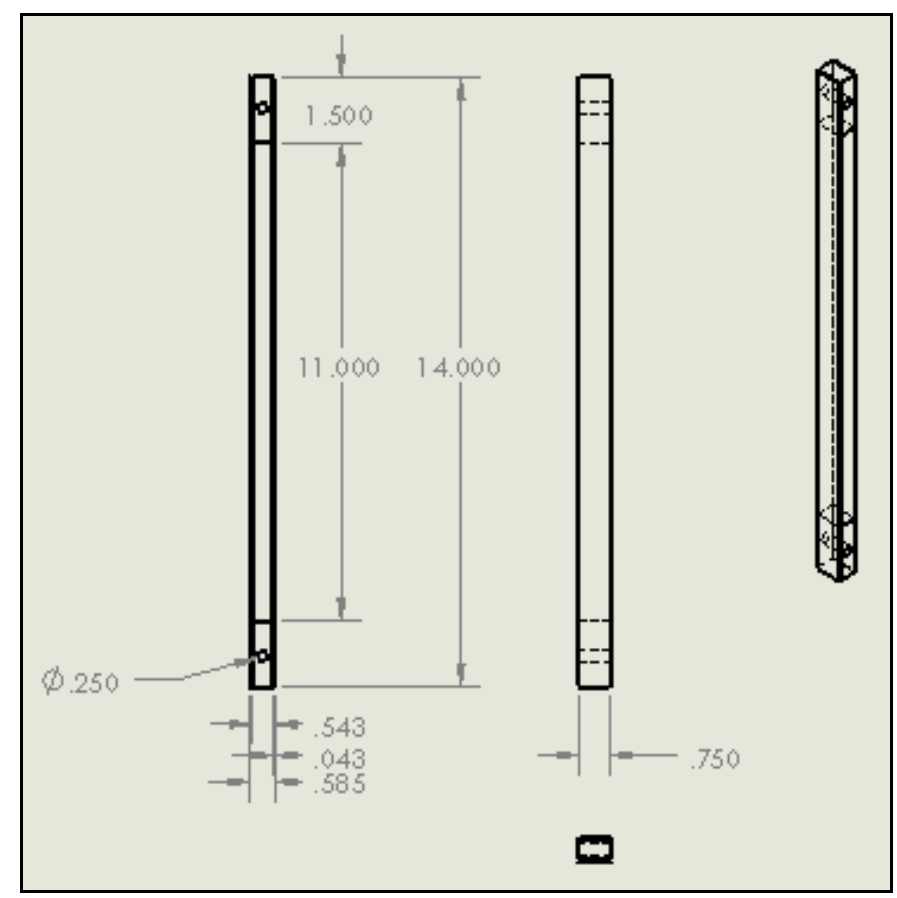

Figure 92: No Delamination and No Embedded Arrestment Key

Control Group: Initial Delamination with No Embedded Arrestment Key - Similar to the previous group, this control group has an initial delamination manufactured into the test specimens. There are different delaminated region sizes that were studied. The area of the delaminated region spans the width of the test specimen and increase in length per half-inch increment starting from 0.5 -inch to 2.0 -inch in length. This particular control group is also compared with the first control group to see its major impact on the composite sandwich structure. 


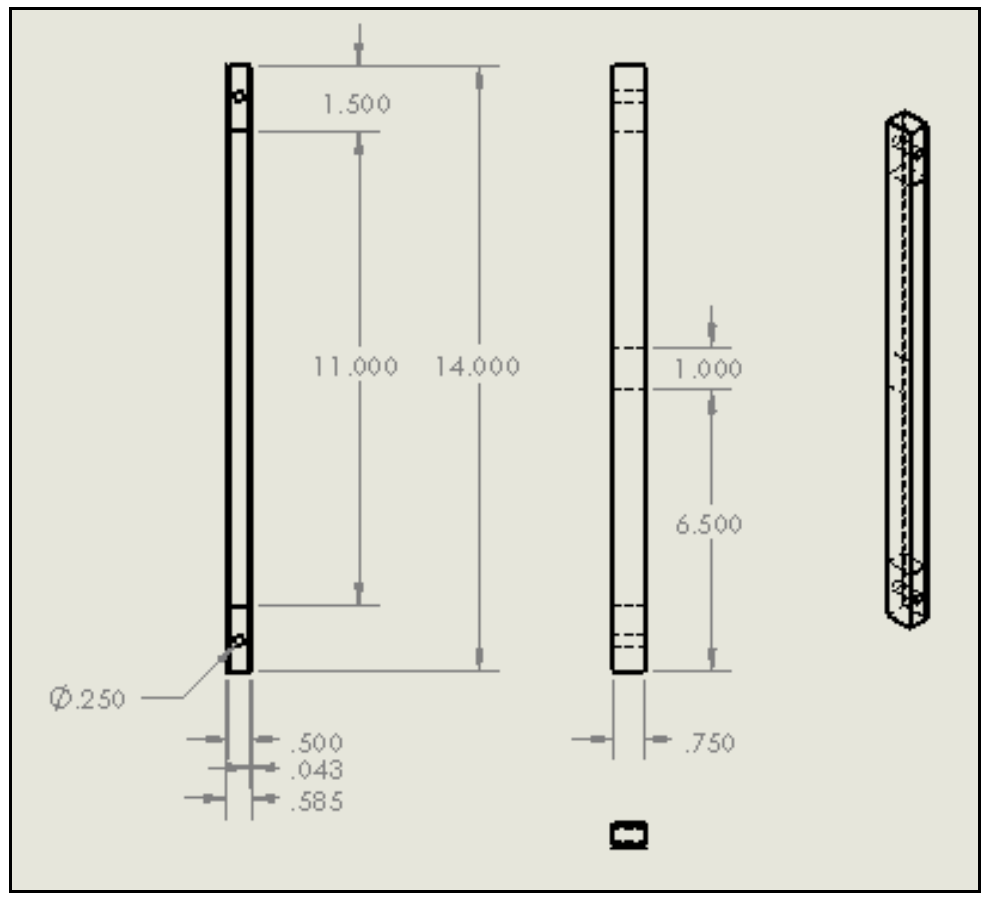

Figure 93: Initial Delamination with No Embedded Arrestment Key

Control Group: No Delamination with Embedded Arrestment Key - The last of the control groups is where the test specimen has no delamination but has an embedded arrestment key that is continuous, centered along the structure, and placed in the vertical direction. This particular control group is also compared with the first control group to see its major impact on the composite sandwich structure. 


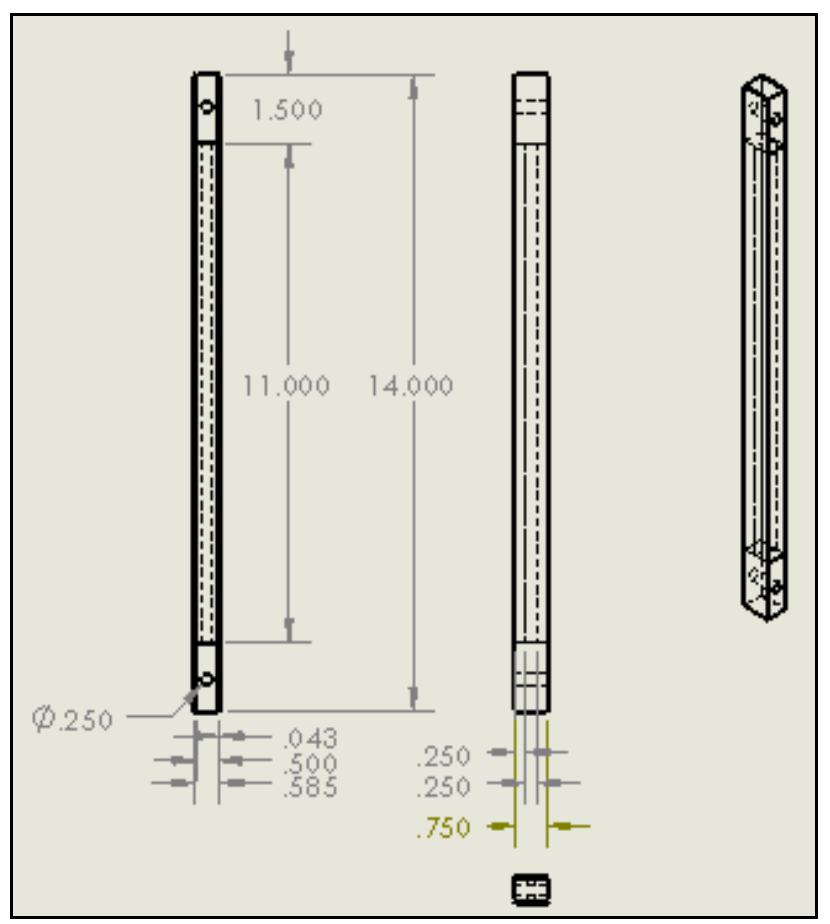

Figure 94: No Delamination with Embedded Arrestment Key

Experimental Group: Continuous Key in Vertical Direction with Skin Delamination -

This experimental group utilizes a continuous key which is placed in the vertical direction in the same fashion as the third control group. The delamination is placed in the same fashion as the second control group where the skin is delaminated from the foam and the key is in the designated region. The particular experimental group only looks into the 1-inch and 2-inch initial delamination cases. 


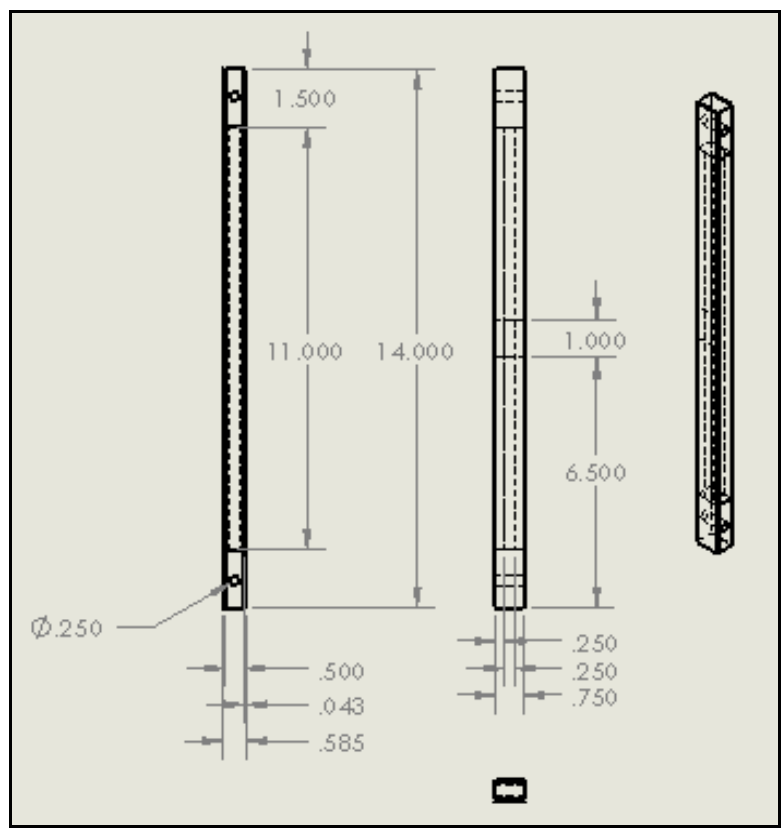

Figure 95: Continuous Key in Vertical Direction with Skin Delamination

Experimental Group: Continuous Key in Vertical Direction with Key Delamination -

This experimental group is similar to the previous experimental groups except the delamination occurs where the key and skin delaminates from the foam. This was studied per 1-inch and 2-inch delamination lengths.

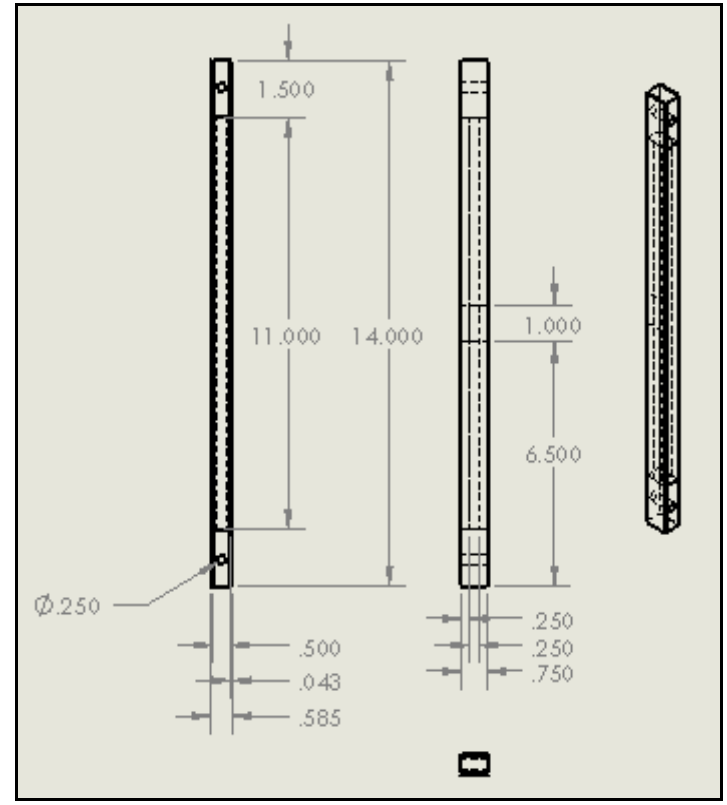

Figure 96: Continuous Key in Vertical Direction with Key Delamination 


\section{Experimental Group: Continuous Key in Horizontal Direction with Skin Delamination}

Configuration - This experimental group is the last of the continuous key configurations but differs where the key is placed in the horizontal direction rather than the vertical direction. There are two keys used in this configuration. The keys extend the width of the composite sandwich structure and have a gap of half an inch from the edge of the delamination area to the edge of the key per each key. This is also studied per 1-inch and 2-inch delamination lengths which mean the keys are shifted to provide the half an inch gap between the delaminated area and the keys.

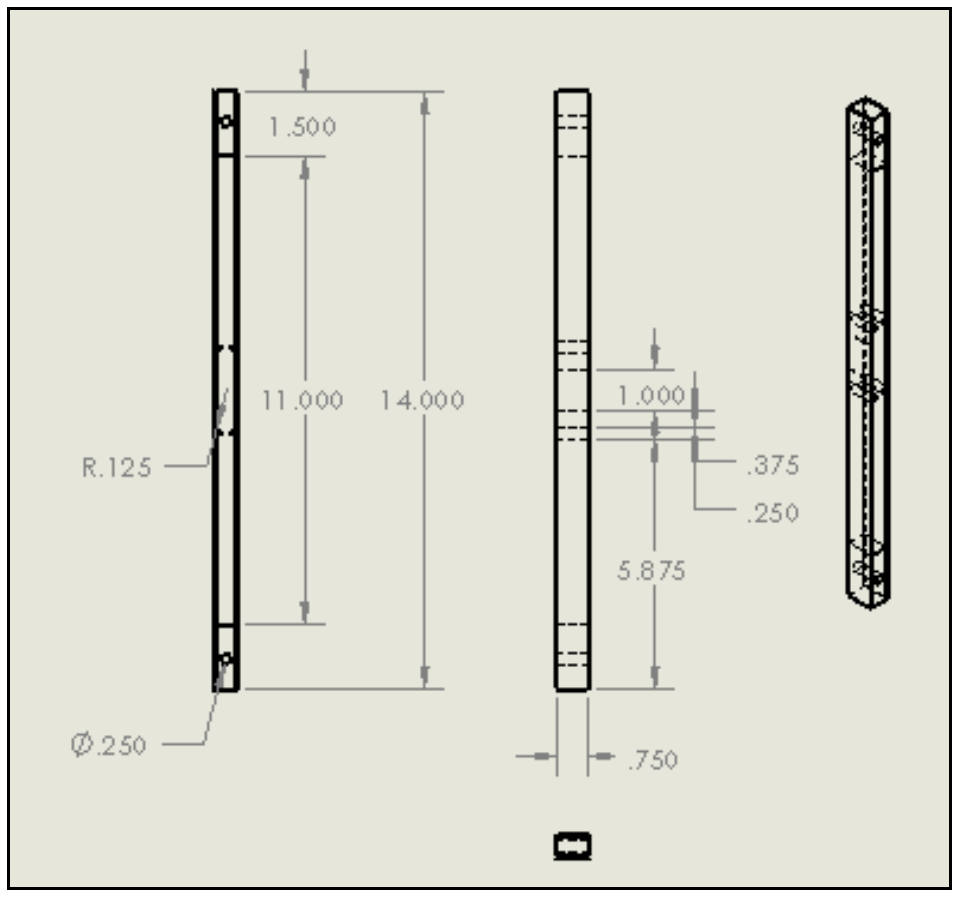

Figure 97: Continuous Key in Horizontal Direction with Skin Delamination Configuration Experimental Group: Discontinuous Key in Vertical Direction with Skin DelaminationThe last of the experimental groups is where the keys are placed in a discontinuous pattern. The pattern is similar to having a continuous key placed in the vertical direction except that there is a break in the continuous key that spans the initial delamination length. 


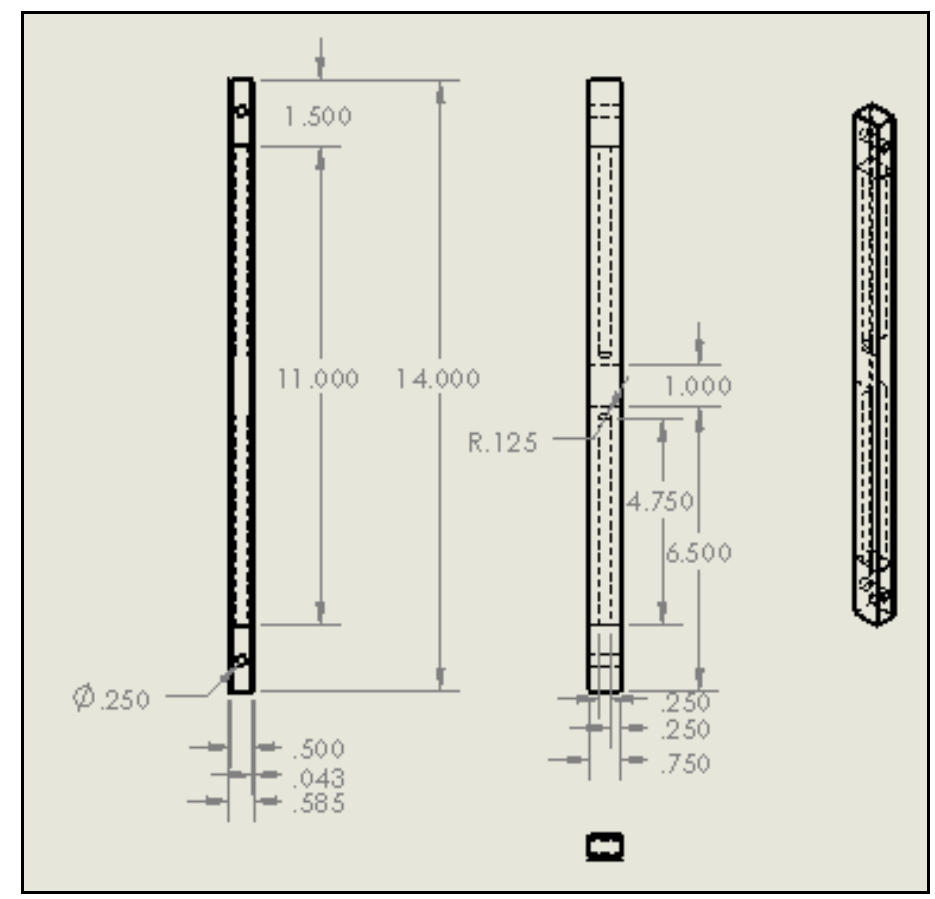

Figure 98: Discontinuous Key in Vertical Direction with Skin Delamination

Another factor that was considered when choosing what configurations to investigate was the direction the key is placed in the composite sandwich structure; that is, whether it is in-plane to the load vector or perpendicular. The other consideration is how the delamination occurs. The most common type of delamination is the skin being separated from the core material; this is also studied. The other type of delamination that was looked into is what if the key delaminated as well from the composite structure. The resultant data will be discussed in the following sections.

\subsubsection{Control Groups}

The control groups, as discussed, are used to provide comparable results between control groups and their experimental group counterparts. The groups include investigating where there is no delamination or an embedded key, test specimens with initial delamination only, and test specimens with an embedded key only.

\subsubsection{Control Group: No Delamination and No Embedded Arrestment Key}

The '11 inches' configuration was retrieved from the buckling length research discussed earlier and is displayed in Table 26. 
Table 26: '11 inches' Configuration

\begin{tabular}{|c|c|c|c|c|}
\hline Case & $\begin{array}{c}\text { Slenderness } \\
\text { Ratio }\end{array}$ & $\begin{array}{c}\text { Max Vertical } \\
\text { Extension }\end{array}$ & $\begin{array}{c}\text { Ultimate } \\
\text { Load }\end{array}$ & $\begin{array}{c}\text { Max Horizontal } \\
\text { Extension }\end{array}$ \\
\hline- & - & in & lbf & in \\
\hline 11 inches & 65.39673 & 0.11083 & 685.60279 & 0.42417 \\
\hline
\end{tabular}

From the Figure 99, the graph shows a gradual climb in load and then begins to round off at the top of the load versus vertical extension until the test specimen experiences a failure. This configuration is built where no additional components in the structure are so that the bonding between the skin and cores is not disturbed.

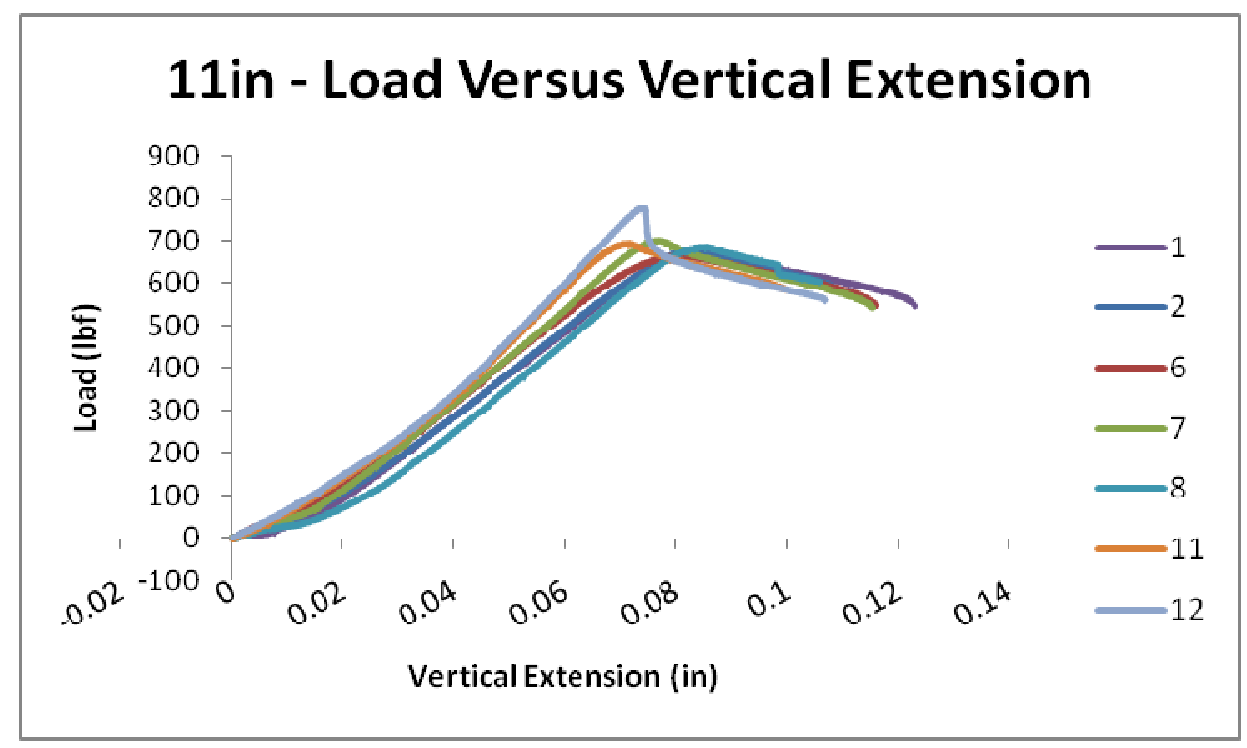

Figure 99: '11 inches' - Load versus Vertical Extension

Figure 100 is quite similar to Figure 99 because the vertical displacement transitioned a displacement in the horizontal direction. This transition is due to the pinned boundaries on the test specimen as it rotates to create a deflection with the maximum extension located at the center. It starts off with a slow increase in horizontal displacement per given loading then begins to exponentially displace. 


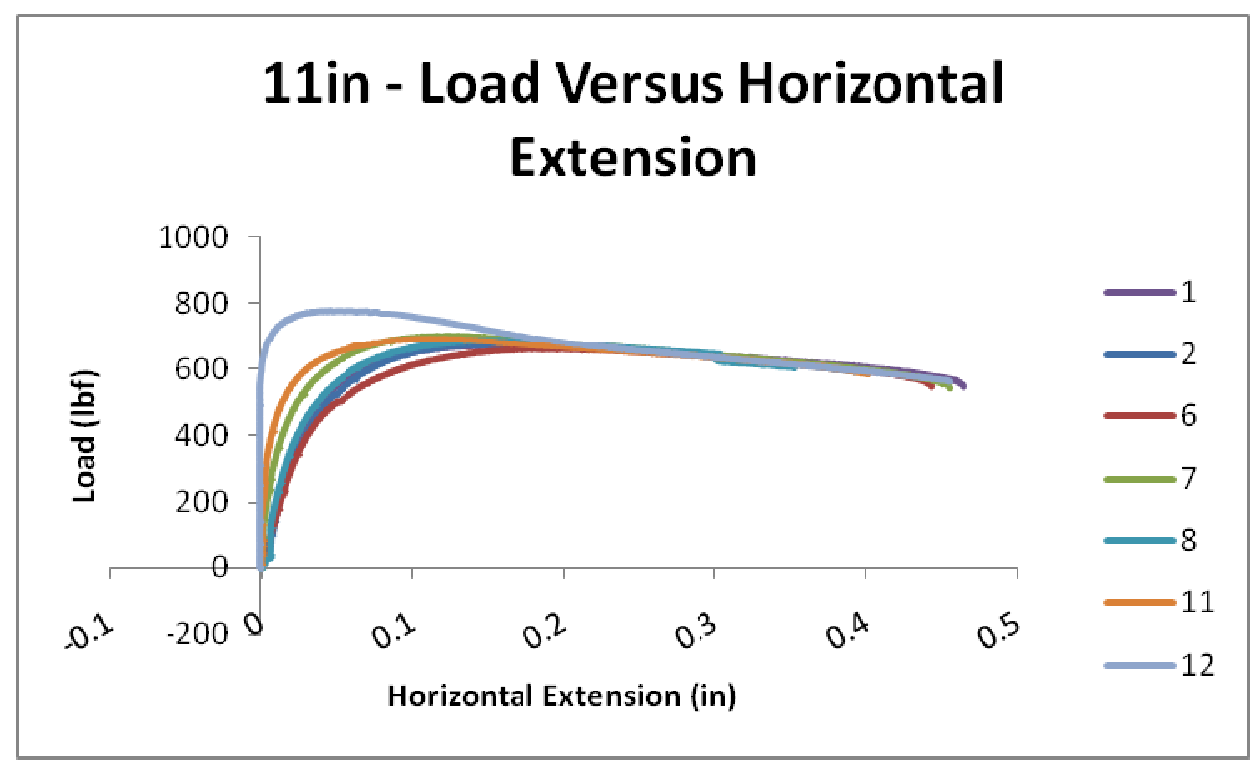

Figure 100: '11 inches' - Load versus Vertical Extension

With the combination of the horizontal extension data and the vertical extension data seen in Figure 101, the graph shows an increased horizontal displacement when the load is applied. This means that the test specimens began to rotate around the pinned points and buckle.

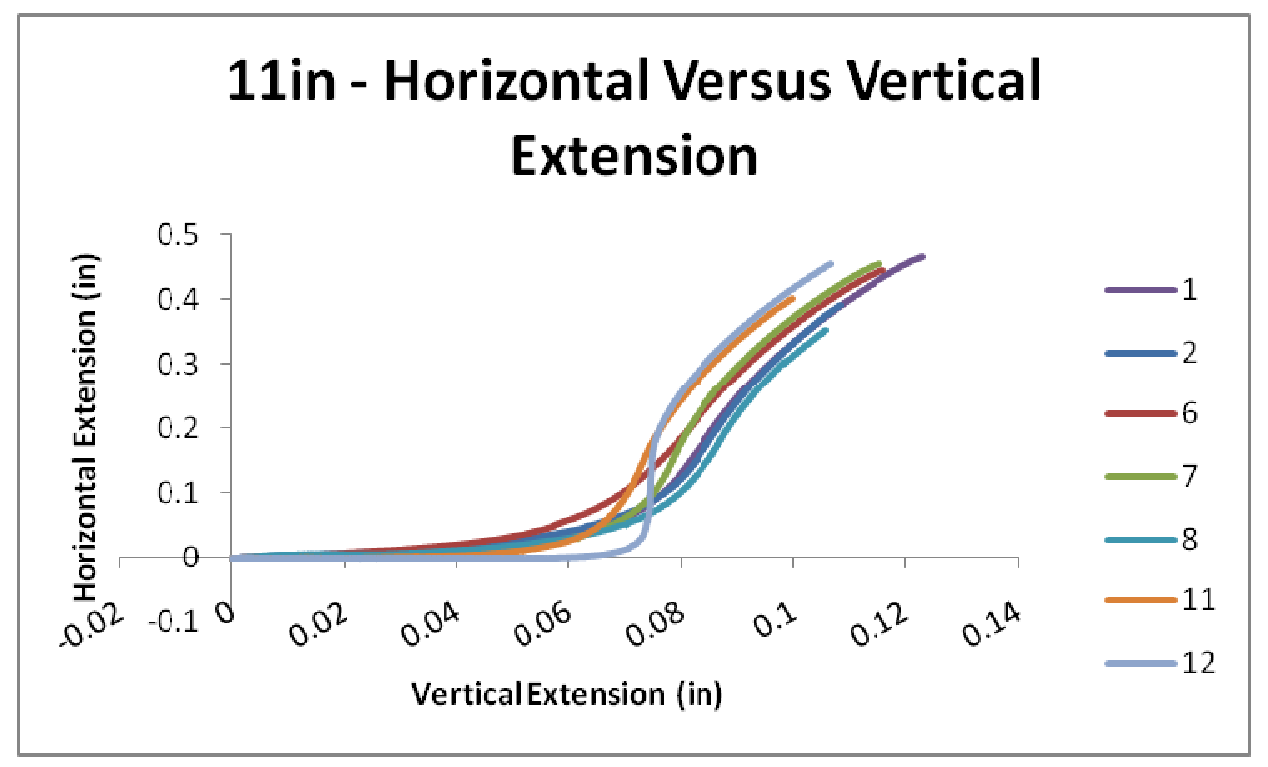

Figure 101: '11 inches' - Load versus Vertical Extension

\subsubsection{Control Group: Initial Delamination with No Embedded Arrestment Key}

The second control group that was studied was test specimens that were introduced with a delamination before they were tested. The introduction of the initial delamination is done with 
non-porous material that is thin and inserted before the skin and the core materials. This nonporous material does not change the material properties of the composite sandwich structure but only create the delamination used in this section. The delamination region extends the width of the structure and varies in height between half-inch to 2 inches in length per half-inch increment. For this group, the delamination length and the term 'ID' denote the different cases after; an example of this is ' 1.0 ID'. The resultant data is shown in Table 27:

Table 27: 'ID’ Initial Delamination Configuration

\begin{tabular}{|c|c|c|c|c|c|}
\hline Case \# & Case & $\begin{array}{c}\text { Slenderness } \\
\text { Ratio }\end{array}$ & $\begin{array}{c}\text { Max } \\
\text { Vertical } \\
\text { Extension }\end{array}$ & $\begin{array}{c}\text { Ultimate } \\
\text { Load }\end{array}$ & $\begin{array}{c}\text { Max } \\
\text { Horizontal } \\
\text { Extension }\end{array}$ \\
\hline- & - & - & in & lbf & in \\
\hline 0 & 11 inches & 65.39673 & 0.11083 & 685.60279 & 0.42417 \\
\hline 1 & 0.5 ID & 66.06927 & 0.05020 & 479.36398 & 0.02274 \\
\hline 2 & 1.0 ID & 65.69982 & 0.03675 & 267.32638 & 0.00986 \\
\hline 3 & 1.5 ID & 66.21160 & 0.02963 & 158.26181 & 0.02399 \\
\hline 4 & 2.0 ID & 65.89214 & 0.04118 & 132.60860 & 0.04519 \\
\hline
\end{tabular}

From the table, it shows that the delamination in the composite sandwich structure dramatically decreases the loading capability of the test specimens. The loading capability drops approximately $35 \%$ for the half an inch delamination, denoted by $0.5 \mathrm{ID}$, and continues to drop per each increase of the delamination's area size. This is why most structures will require repairs before the delamination can affect the structural integrity and needs frequent inspection.

Otherwise, the structure can easily become an expensive piece of scrap. 


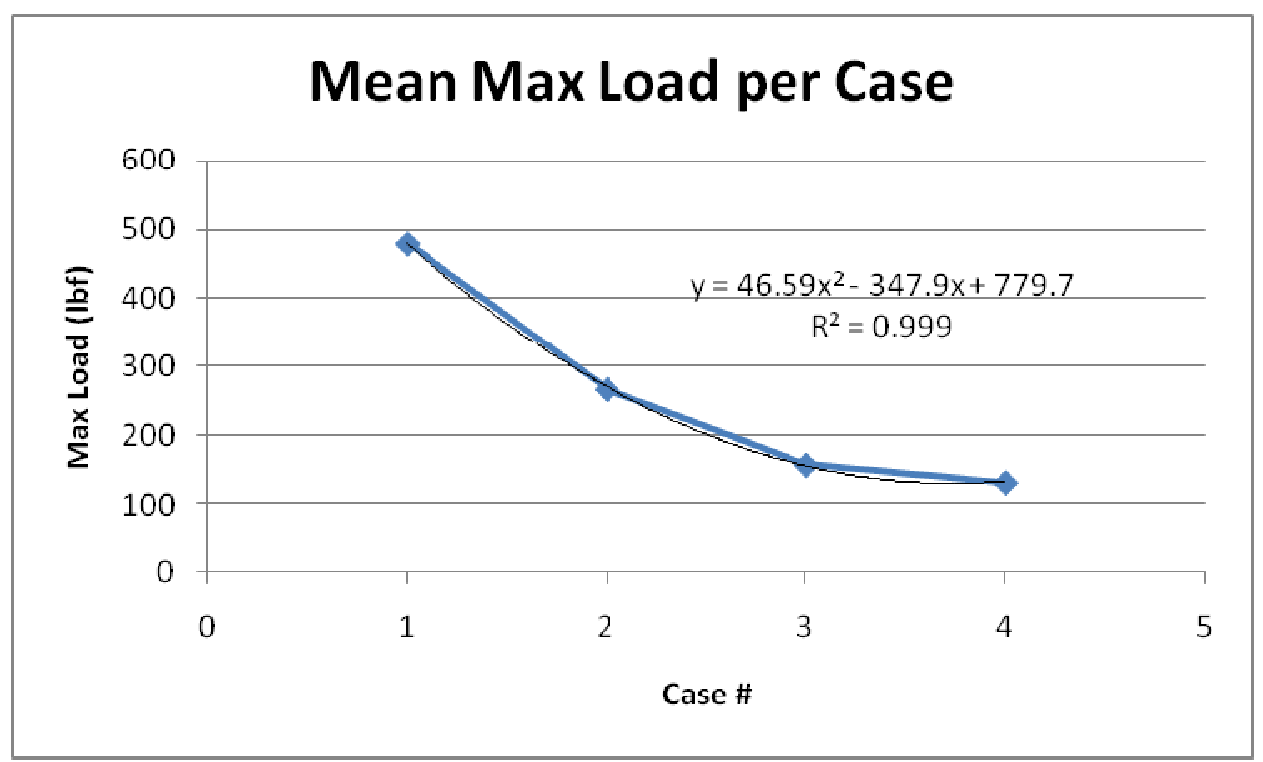

Figure 102: Mean Ultimate Load per Case

Using the data from Table 27, the graph shows decrease in the load capacity as the delamination region increases. This is a typical trend for most composite metals. One thing to note about Figure 102 is that it fits a parabolic trend line and seems to become asymptotic as the size of the delamination increases. As for Figure 103, it shows a similar trend as Figure 102 but the difference is the geometry factor.

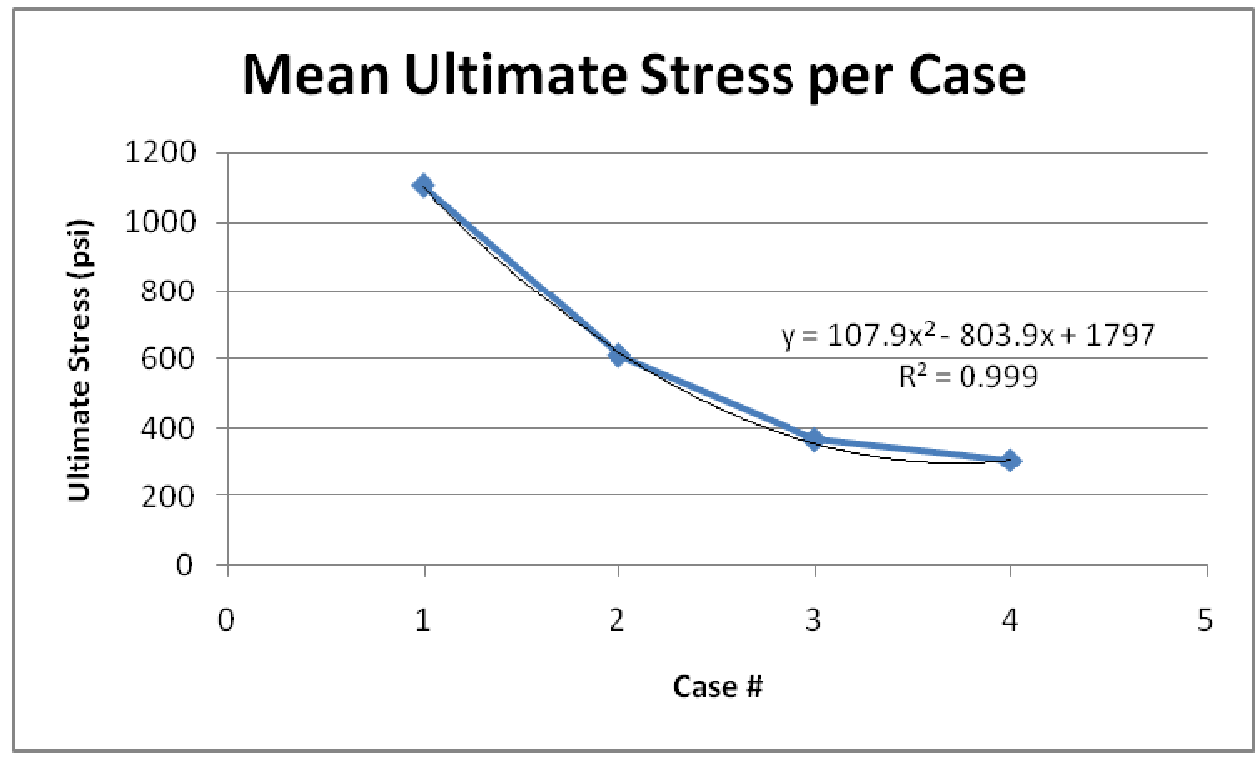

Figure 103: Mean Ultimate Stress per Case 


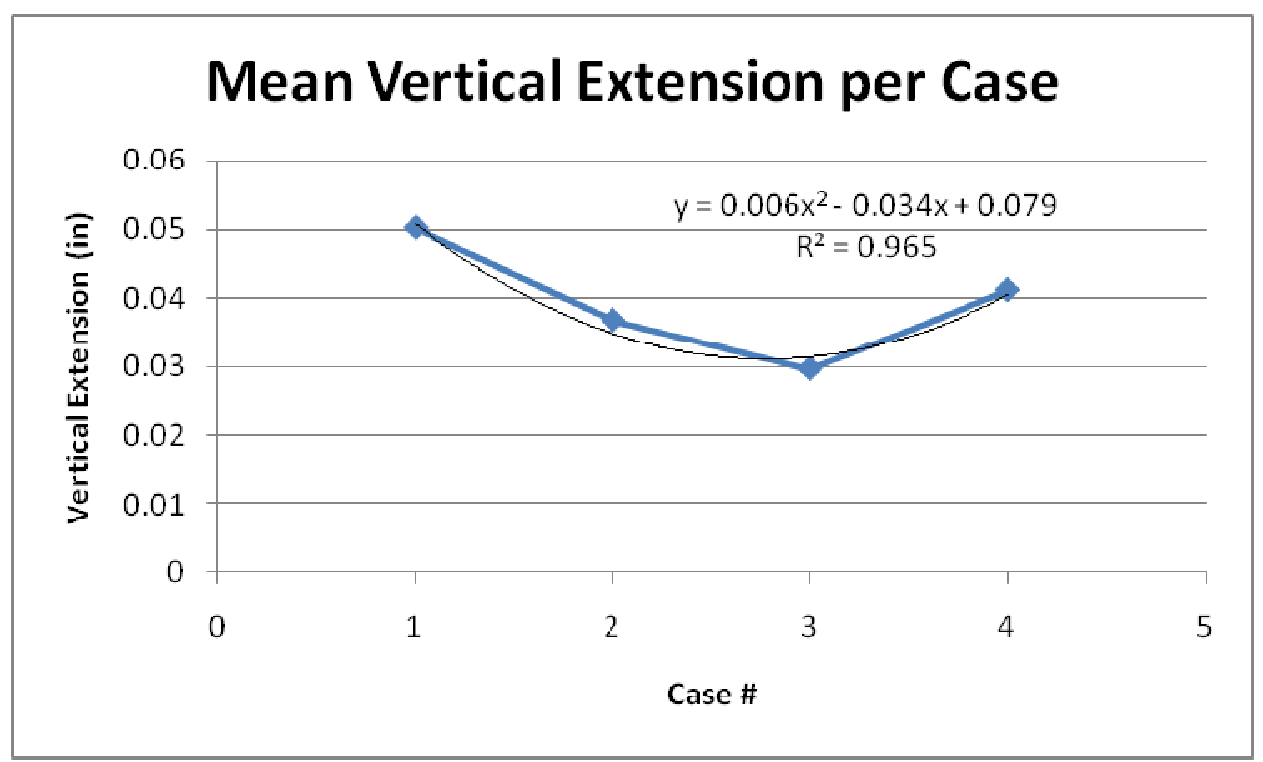

Figure 104: Mean of Maximum Vertical Extension per Case

The maximum vertical extension per each of the initial delamination cases is shown in

Figure 104. The data shows that the extension is greatest with the 0.5 - inch delamination and bottoms out at the 1.5-inch delamination test specimen. From this parabolic trend line, the increase in the size of the delamination means a decrease in vertical extension until the curve bottoms out and becomes asymptotic. The reason for the decrease in vertical extension with the increase in delamination size is because the composite sandwich structure fails much earlier. The 2.0-inch case should be disregard from the graph because the vertical extension during the data acquisition was not properly set up.

As the vertical extension decreases, the horizontal extension increases due to the structural incapacity to resist the flexure as the structure is loaded. Figure 105 details the results of the horizontal extension per each case. The case regarding the 0.5 -inch delamination was removed from the graph due to its high standard deviation. This high standard deviation can be attributed to the data acquisition setup process. 


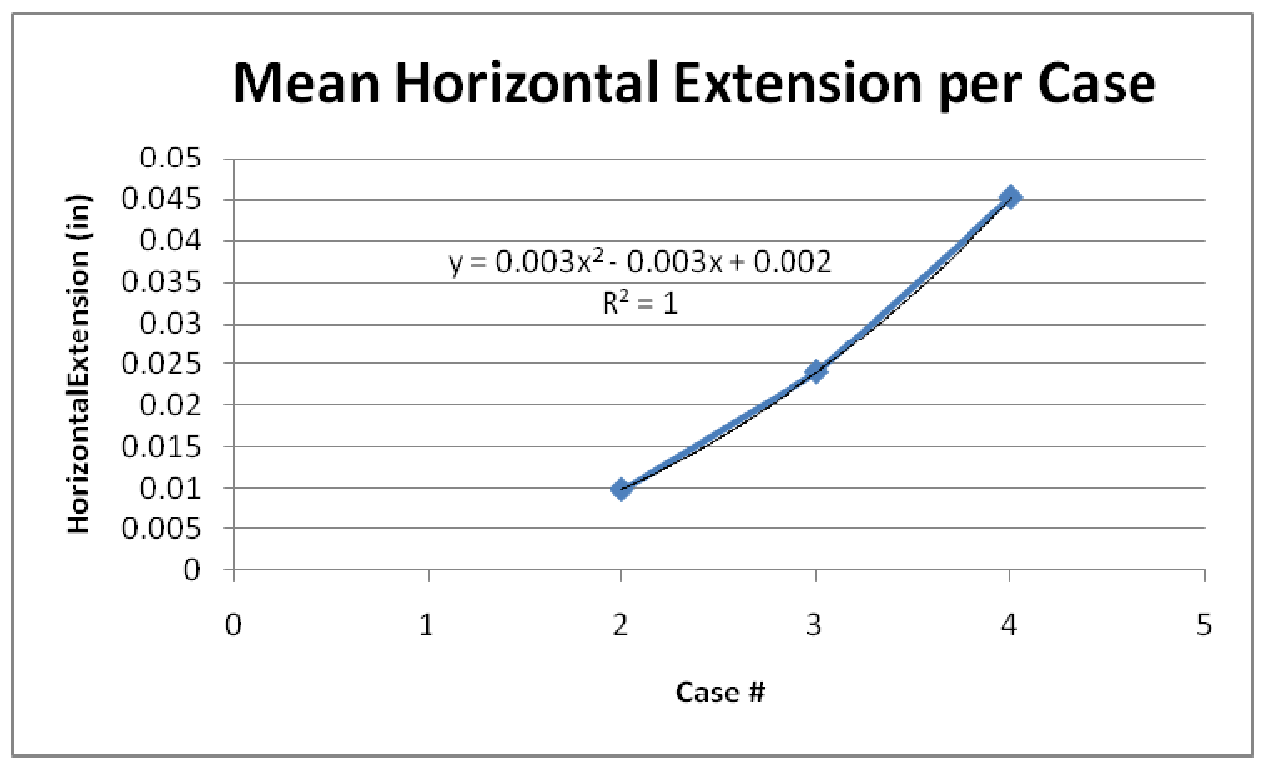

Figure 105: Mean of Maximum Vertical Extension per Case

Note that the following subsections show individual cases with the various delamination sizes. The test specimens in these subsections have the same geometry and same manufactured process except for the delamination sizes. The buckling of these test specimens is only measured on the side with the delamination region. The issue with the buckling of the structure and face sheet is that it requires two different extensometers to measure the extensions on both sides of the test specimens. With the limitations in resources, one extensometer was used and placed on the delamination region side of the test specimens.

\subsection{Half-Inch Initial Delamination}

The first delamination set of data comes from the test specimens that have an initial delamination that extends the width dimension and the 0.5 -inch length at the center of the test specimen. The test specimens remain elastic in nature until they fracture. This is detailed in Figure 106 where the test specimens are introduced to in-plane loading and remain in the elastic region. The main reason for the brittleness of the structure comes from the materials chosen. The foam core is quite brittle in nature and is reinforced with fiberglass skin. 


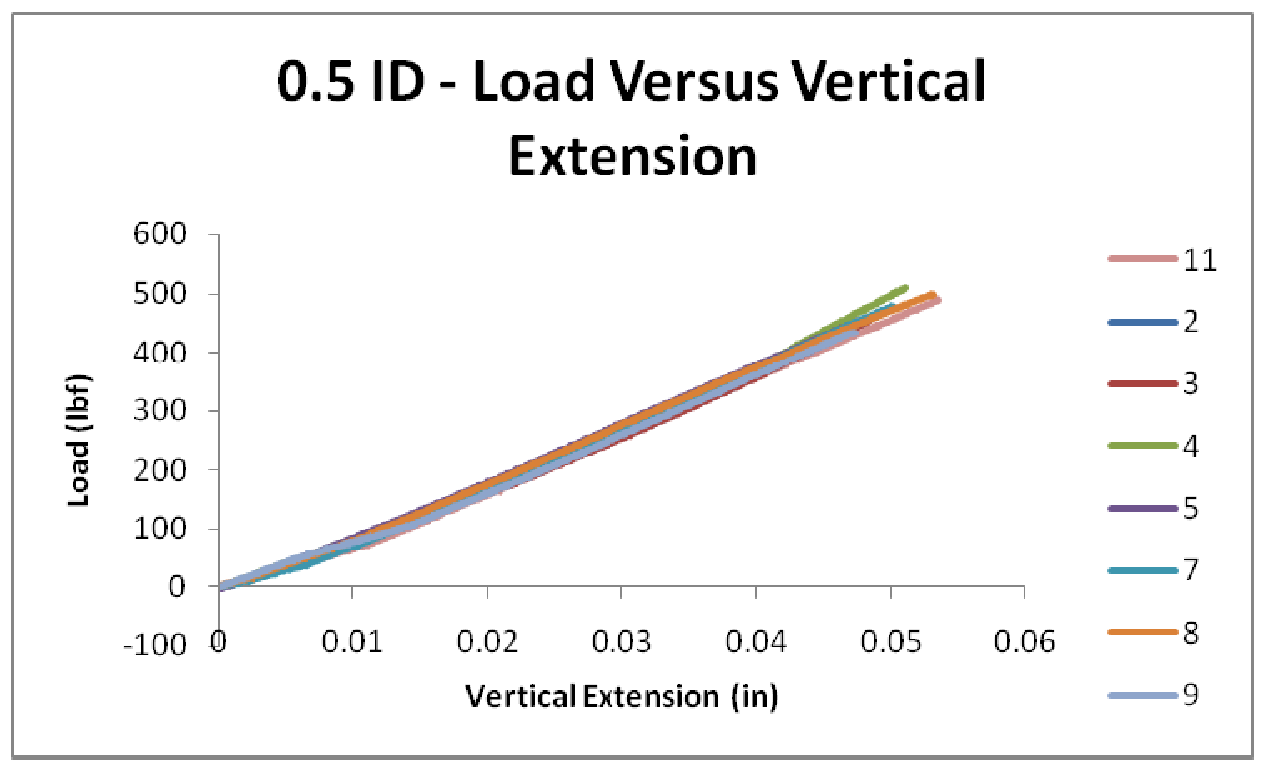

Figure 106: 0.5 ID - Load Vs Vertical Extension

Figure 107 shows the test specimens extending horizontally and the buckling of the face sheet before it fractures. This reflects how well the test specimens can hold the load conditions.

The smaller the delamination region, the higher loading capacity of the test specimen becomes.

This is due to less localized stresses on the part. Figure 107 looks odd due to the limitation of the extensometer causing the higher fluctuation.

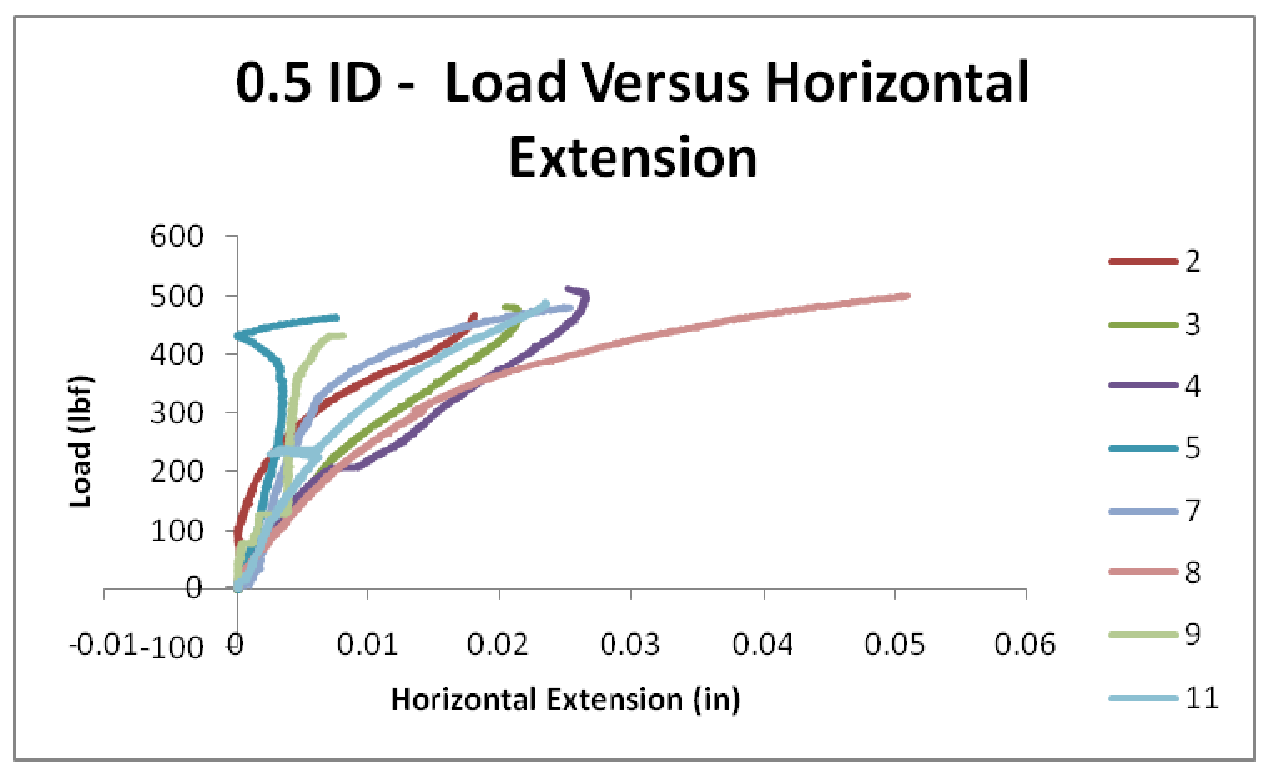

Figure 107: 0.5 ID - Load Vs Horizontal Extension 
Figure 108 represents the relationship between the horizontal extension and vertical extension and shows a progressive increase in horizontal extension as the vertical extension is being displaced. There is much more vertical displacement than horizontal displacement hence the low profile curves in the figure. This is seen as the little spike at the end of the plot.

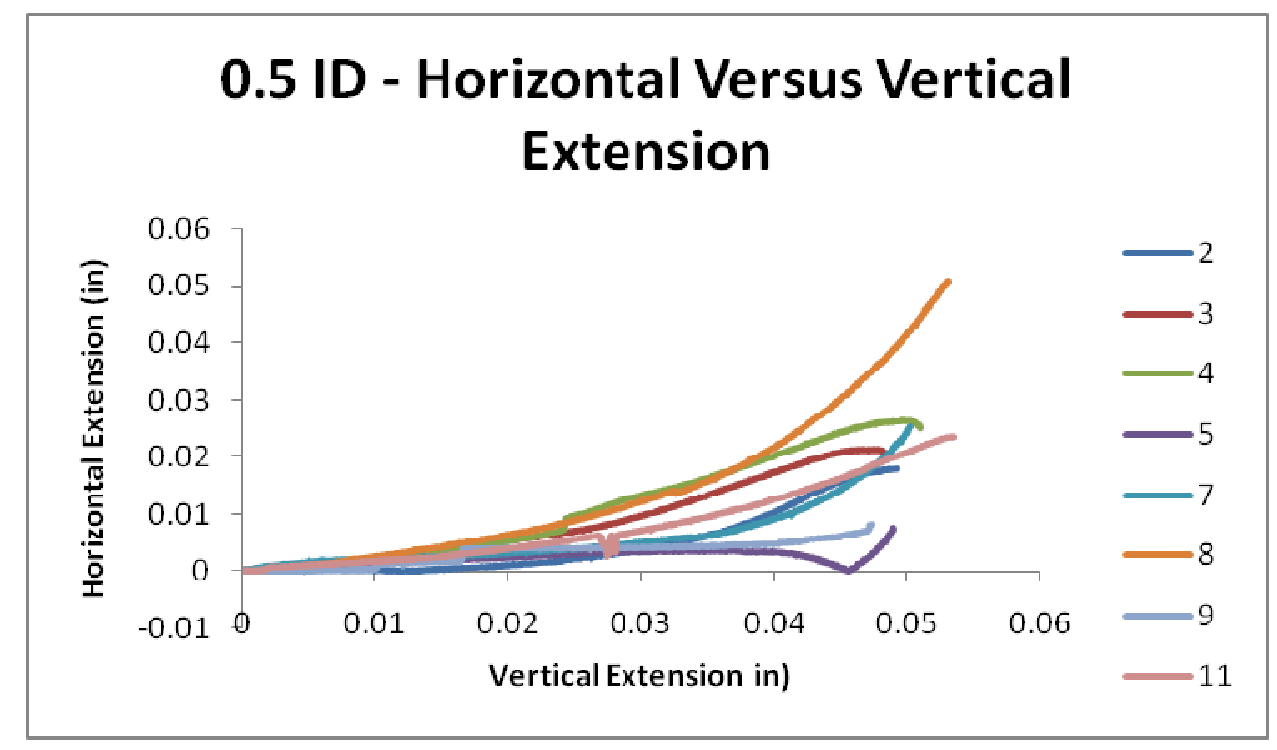

Figure 108: 0.5 ID - Horizontal Extension Vs Vertical Extension

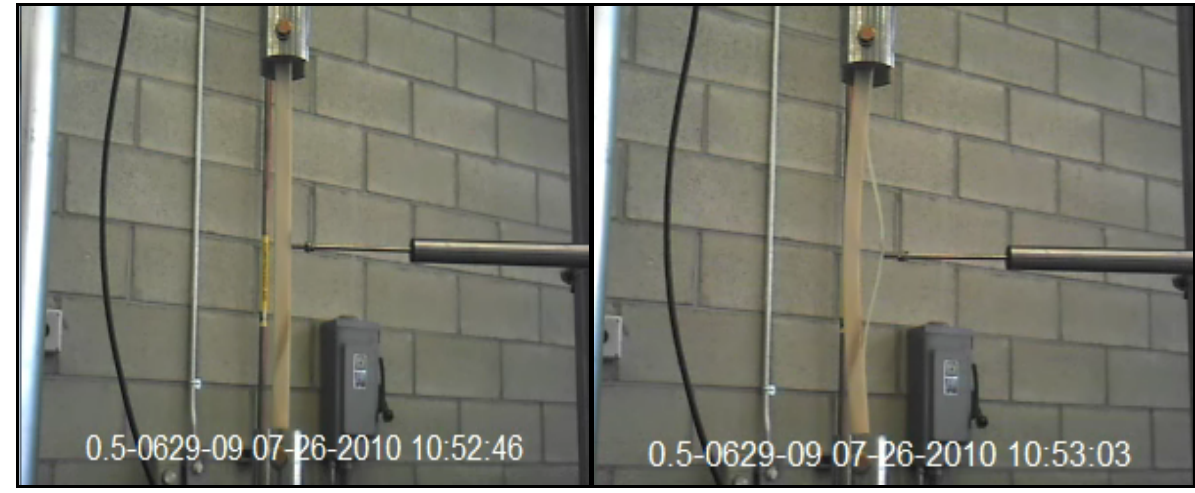

Figure 109: 0.5 ID - Experimental Testing Before and After Failure

What can be seen from Figure 109 was that the experimental testing before failure shows very little horizontal extension and as the strain energy releases, the crack propagates very quickly through the test specimen as seen on the right image. 


\subsection{1-Inch Initial Delamination}

The second data set comes from the 1.0-inch delamination test specimens that were tested under in-plane loading. What differs in Figure 110 in comparison to the 0.5 -inch delamination is that the test specimens fail a bit earlier thus the reason for the shorter vertical extension. The test specimens still exhibit the same elastic properties, but begin to transition near the end of life before they fracture as seen in Figure 110.

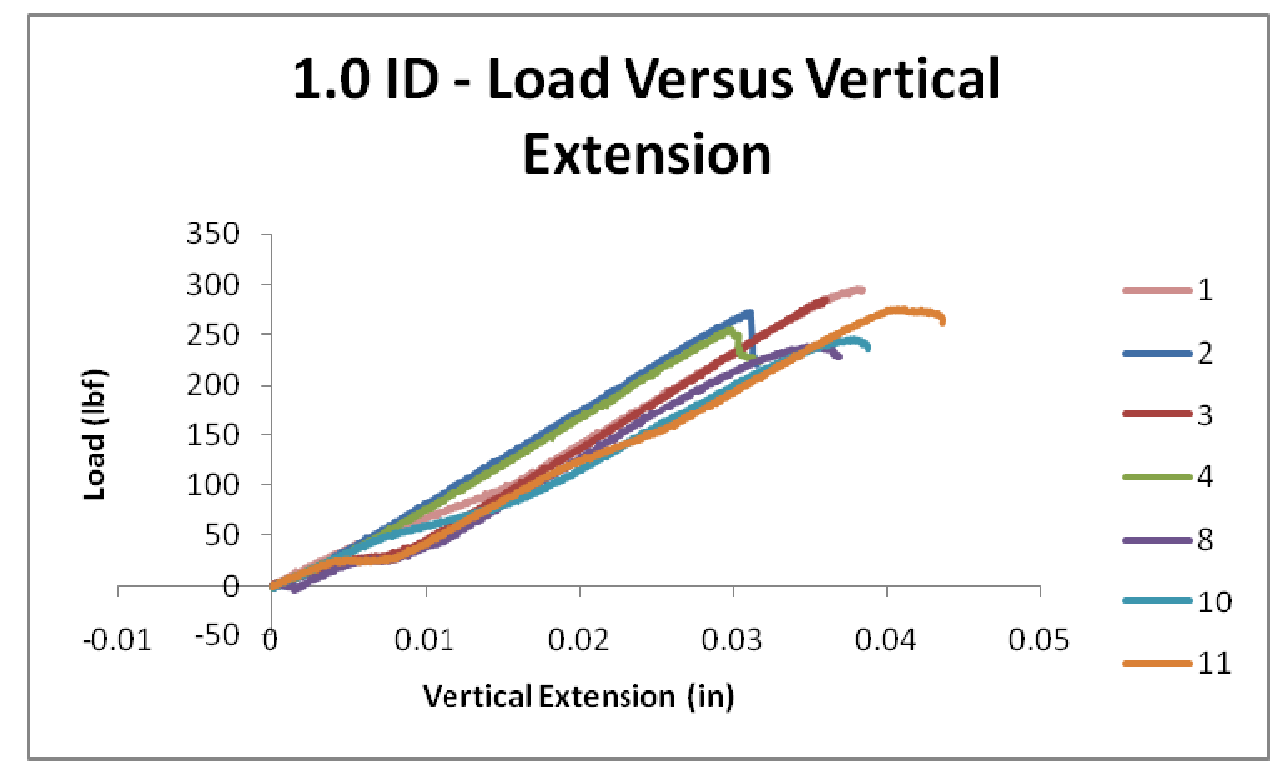

Figure 110: 1.0 ID - Load Vs Vertical Extension

In Figure 111, it shows a trend that deviates and transitions from the vertical extension where the test specimens would see a slight buckling occurrence of the face sheet when the loading is closer to the ultimate. As discussed earlier in ' 0.5 ID' configuration section, the limitation of the extensometer dramatically affects the deviation of the horizontal displacement. What can be extract from the graph is that the test specimens require less loading when increased in delamination length. 


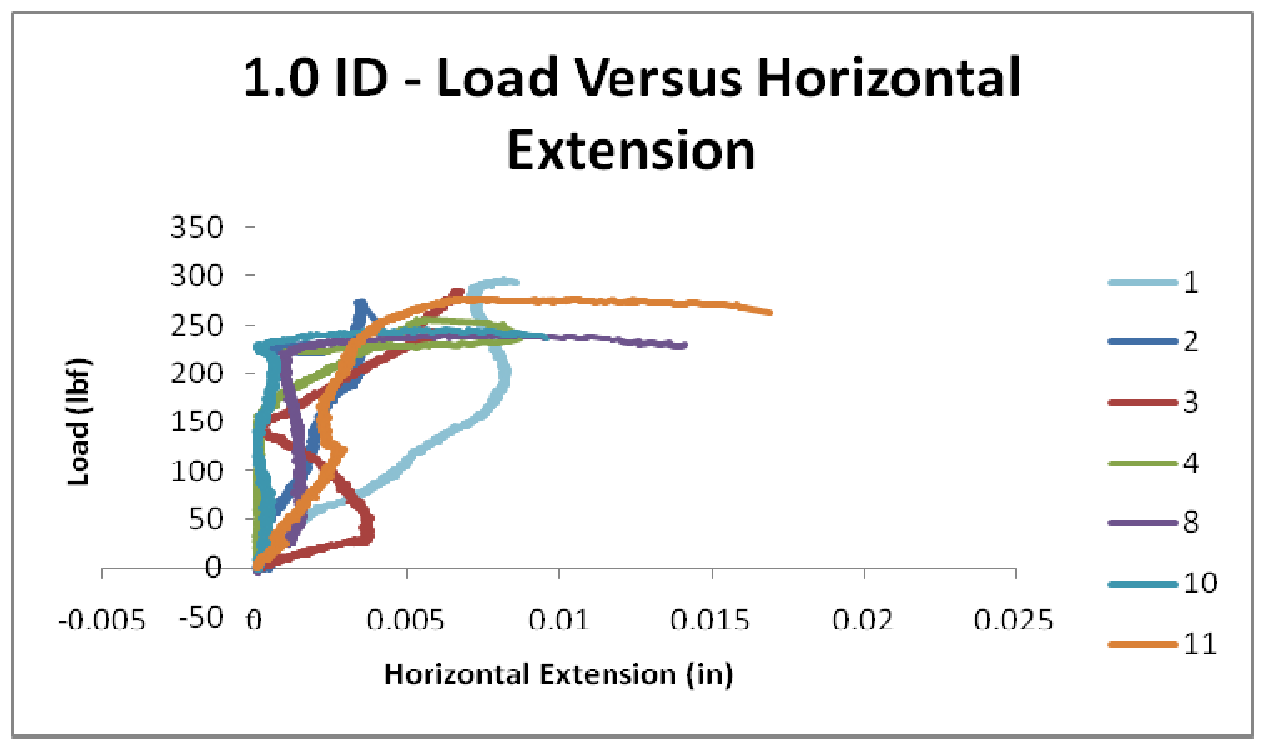

Figure 111: 1.0 ID - Load Vs Horizontal Extension

From the combination of the vertical extension with the horizontal extension data, it does show that the skin begins to buckle but the structure would fail before the initial delamination could do more damage. This is shown in Figure 112.

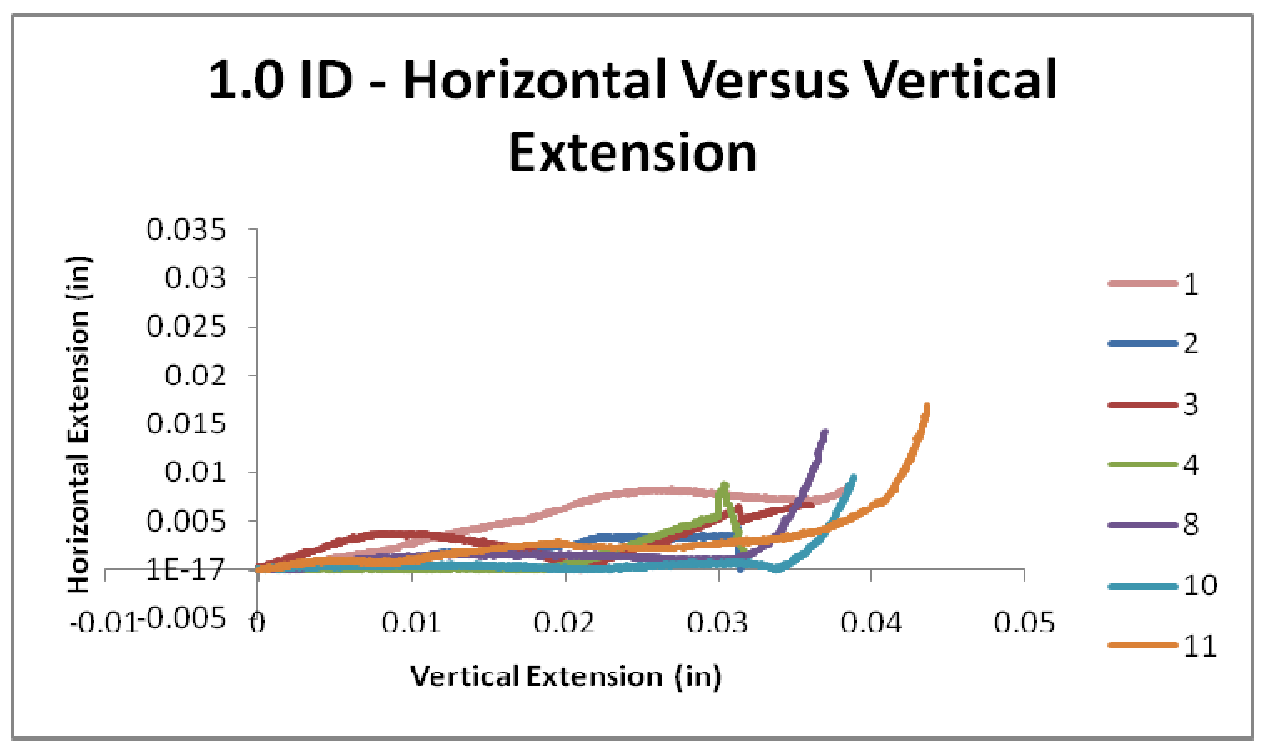

Figure 112: 1.0 ID - Horizontal Extension Vs Vertical Extension 


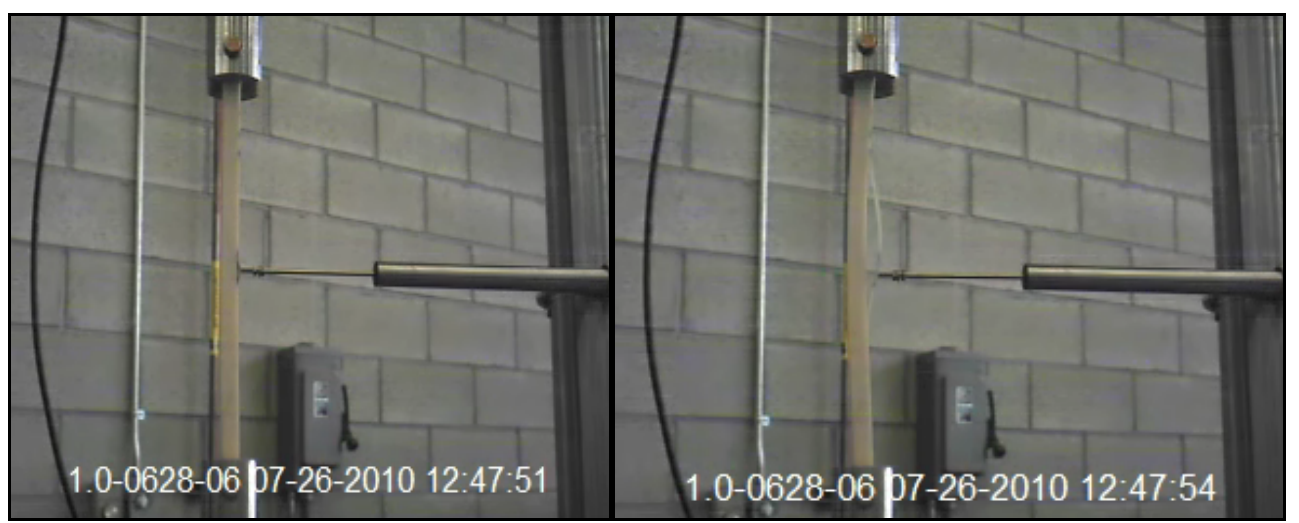

Figure 113: 1.0 ID - Experimental Testing Before and After Failure

Similar to what was seen for the ' 0.5 ID' configuration, the ' 1.0 ID' configuration differed by having a slightly larger extension. The strain energy also propagated the crack and caused the failure of the test specimen. This can be seen in Figure 113.

\subsection{5-Inch Delamination}

As for the 1.5-inch delamination test specimens, the delamination of these test specimens is buckling more than the previous two delamination cases. The buckling occurred because the delamination region is much larger and the edge of the delamination region, or the crack, propagates between the skin and core under in-plane loading. The Instron displacement machine will stop the test when the test specimens exhibit a $40 \%$ load drops from the ultimate loading. This is why Figure 114 shows a failure point at the largest vertical extension. Also, another thing to note is how the load versus vertical extension is curved. The curvatures show a displacement that requires less loading, which is exhibiting instability in the composite sandwich structure. 


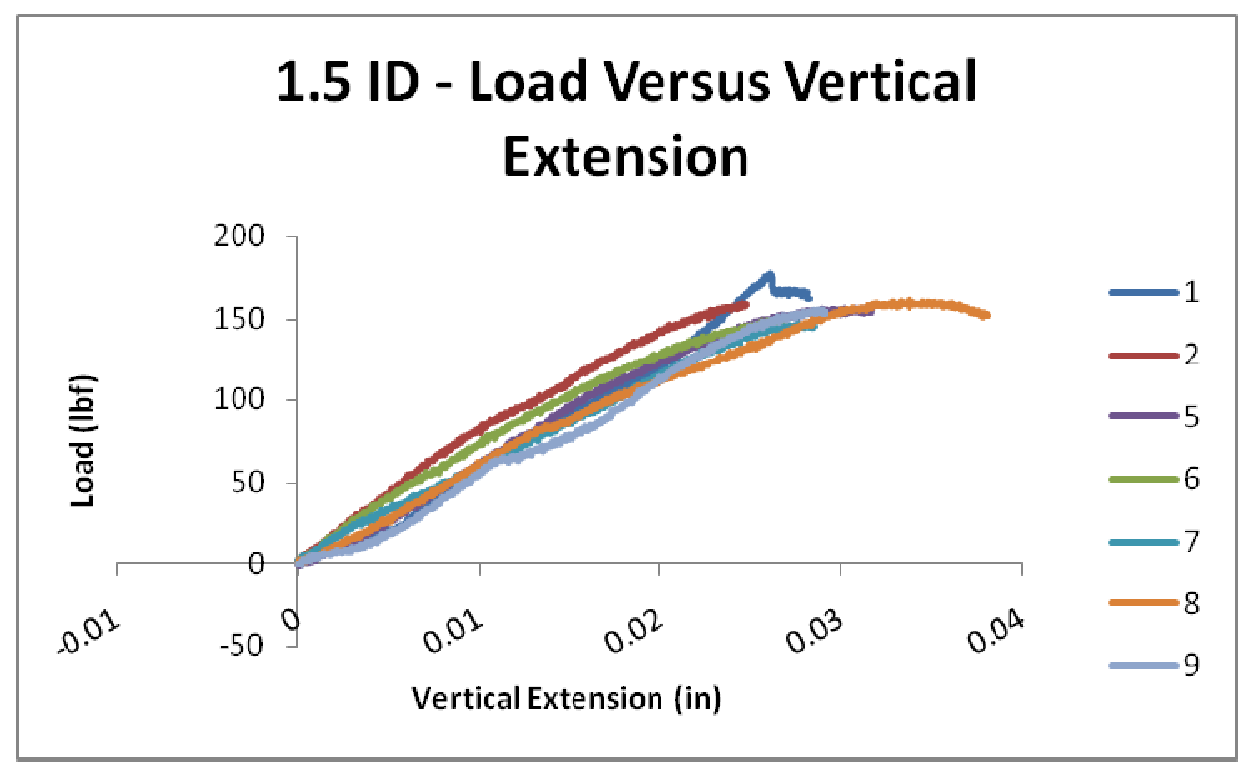

Figure 114: 1.5 ID - Load Vs Vertical Extension

As seen in Figure 115, the load versus horizontal extension transitioned from Figure 114.

The vertical extension is transitioned to the horizontal direction where it slows displacement in the horizontal direction with the increase in loading. The curvature in each of the curves details the test specimen's buckling of the whole structure in one direction. Then the hook in the curves details the horizontal extension of the face sheet in the opposite direction.

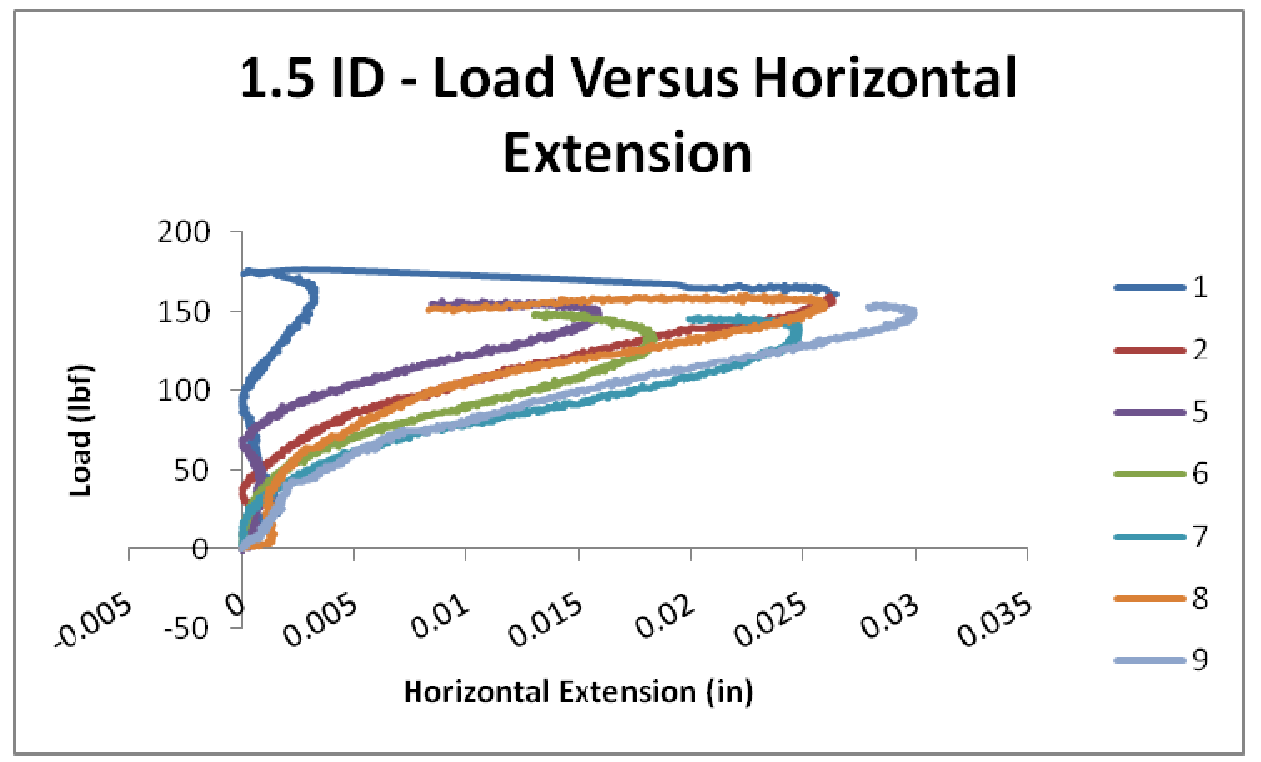

Figure 115: 1.5 ID - Load Vs Horizontal Extension 
The combination of the two extensions, which are the vertical and horizontal extensions, shows a great amount of horizontal extension as the vertical extension increases. Same as what has been discussed; the face sheet delamination buckles more with the increase size of the delamination. This figure below details that quite well.

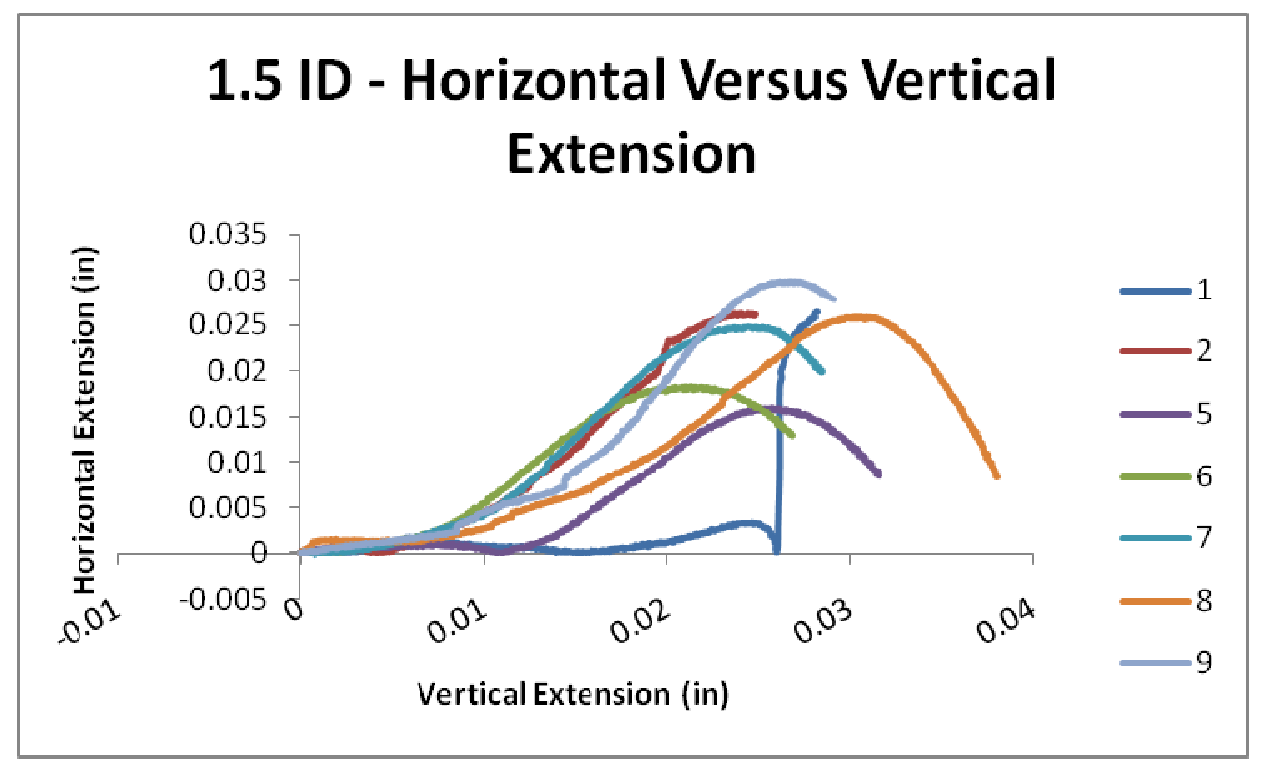

Figure 116: 1.5 ID - Load Vs Horizontal Extension

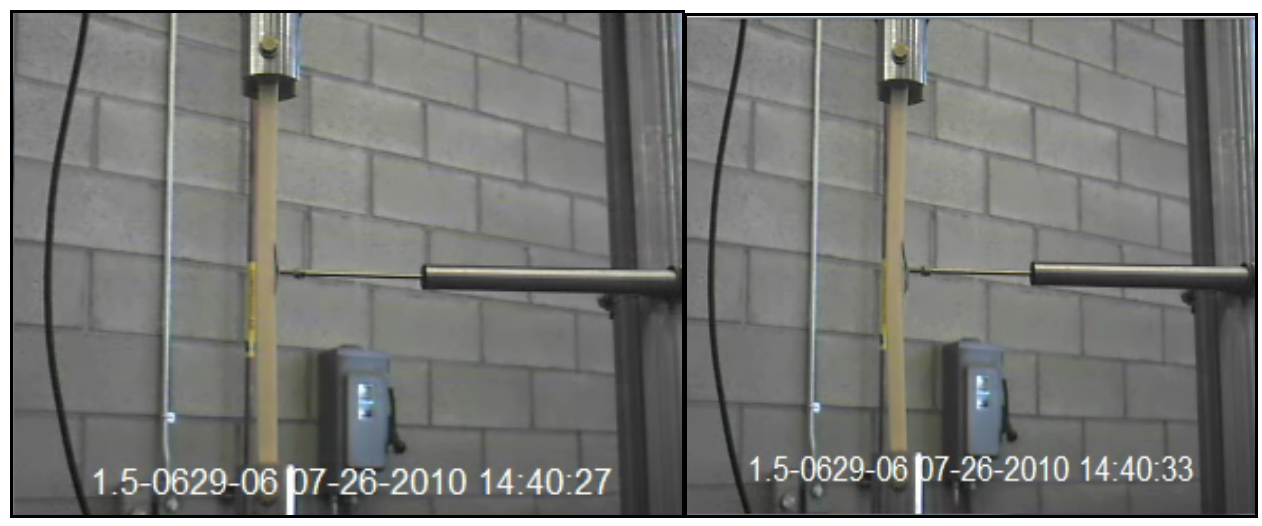

Figure 117: 1.5 ID - Experimental Testing Before and After Failure

As the initial delamination size starts to increase, the face sheet delamination becomes more visible and can be seen in Figure 117 on the left. The right image shows the failure of the test specimen and because of the larger delamination size. There is less strain energy causing the crack propagation to not spike as high along the seam between the foam and fiberglass skin. 


\subsection{2-Inch Delamination}

Similar to all the other subsections discussing the transition of the composite sandwich structures to the increased experience of a buckling feature; the 2-inch delamination region experiences this most and much earlier due to the delamination size and the ease of propagation.

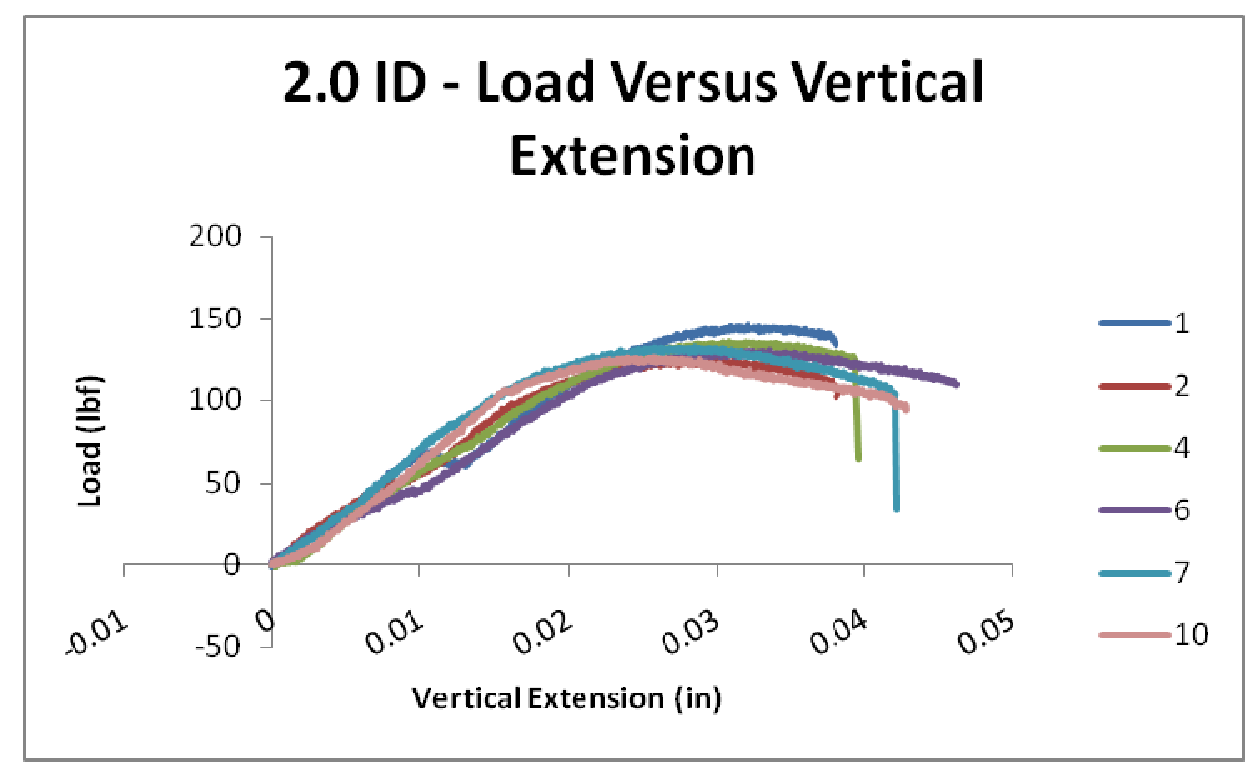

Figure 118: 2.0 ID - Load Vs Vertical Extension

Similar to what was discussed for '1.0 ID' configuration regarding the load versus the horizontal extension, this stands true for the '2.0 ID' configuration as well. The only difference shown in Figure 119 is the increase how much the face sheet is displaced as the vertical displacement is applied. These two graphs show a lot of similarities where the structure buckles in one direction as the face sheet buckles in the other direction. 


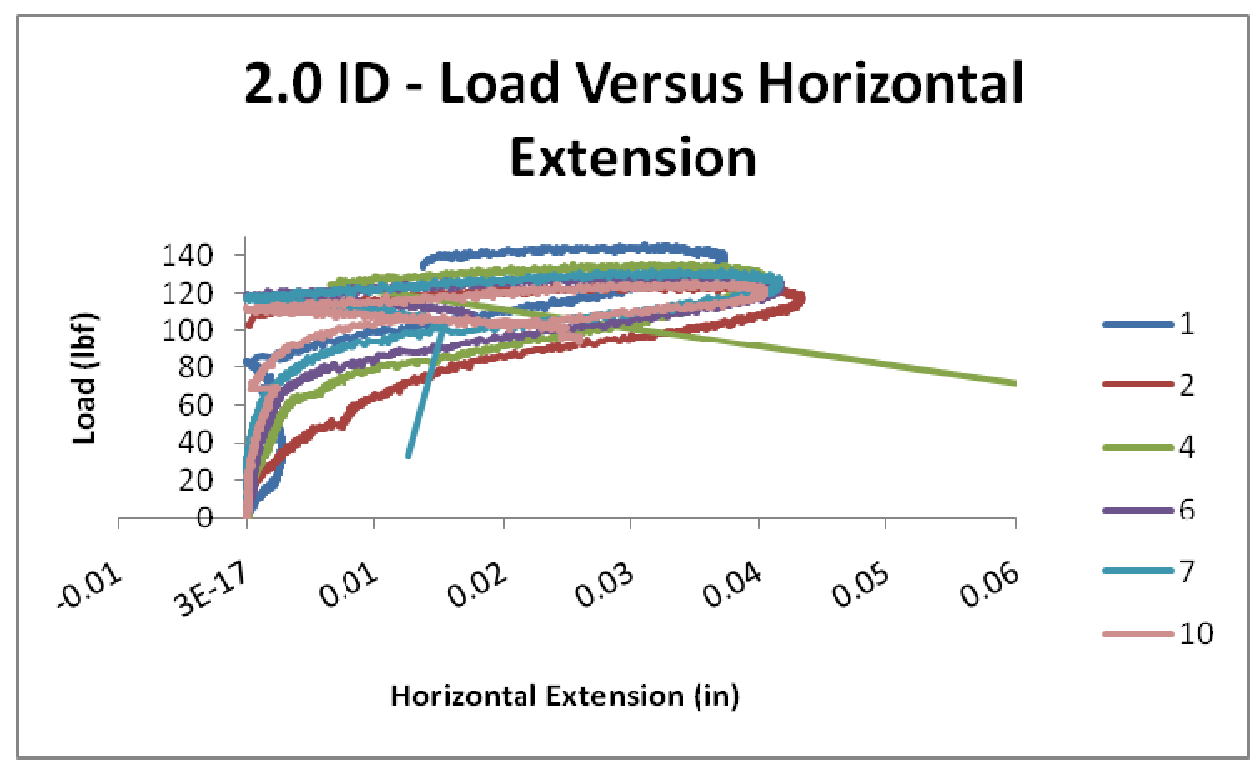

Figure 119: 2.0 ID - Load Vs Horizontal Extension

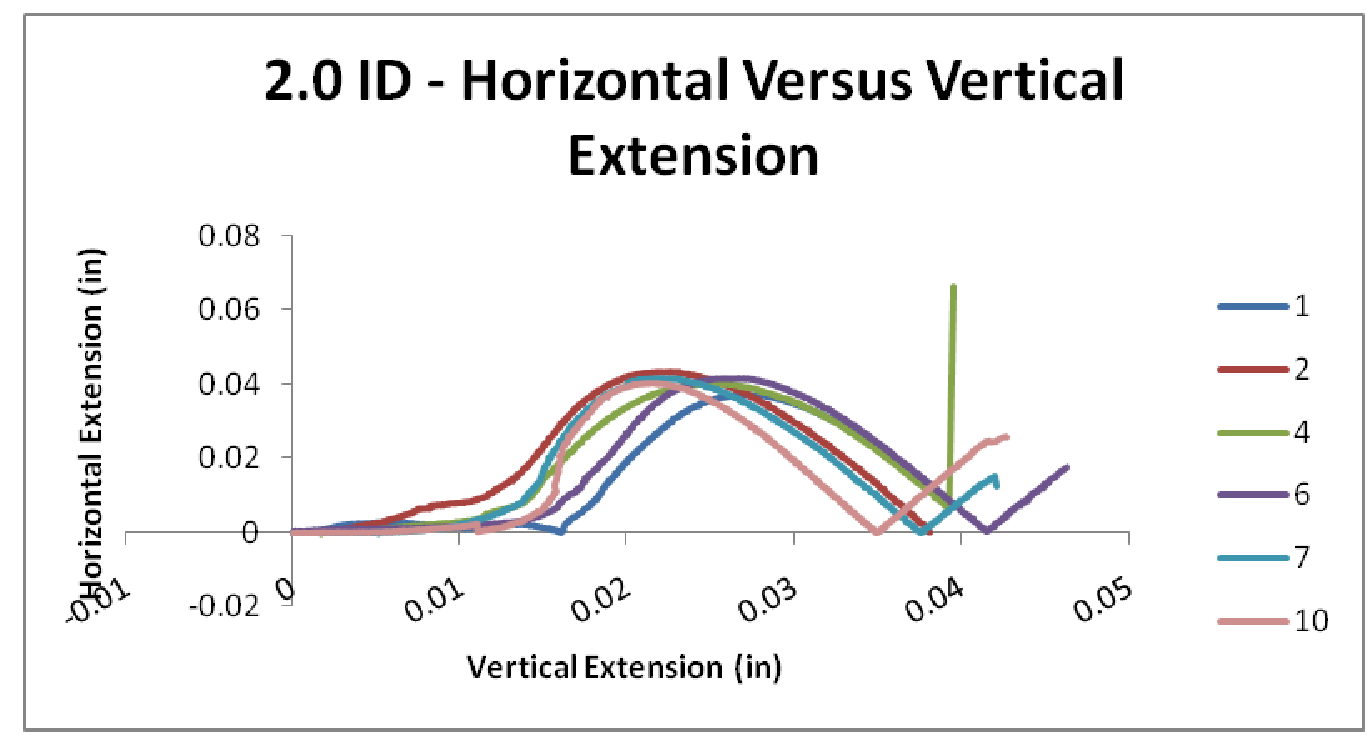

Figure 120: 2.0 ID - Horizontal Extension Vs Vertical Extension

In Figure 120, the trend is a bit different because the structure buckles much more before the face sheet begins to buckle. This is seen in the graph where the horizontal begins to climb then recedes as the vertical extension increases. The spikes at the end of the curves in Figure 120 are the points of failure. 


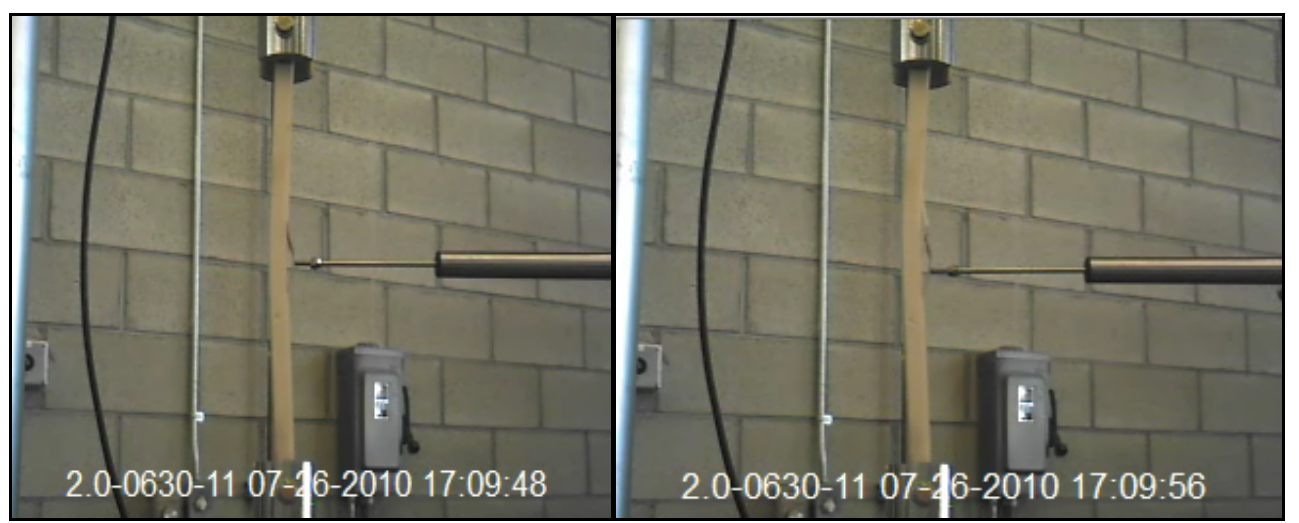

Figure 121: 2.0 ID - Experimental Testing Before and After Failure

Figure 121 shows a longer horizontal extension because of the initial delamination size

placed under loading. This later transitioned to a small burst of energy causing the edge of the initial delamination to propagate a bit more. The larger delamination size also shows a larger decrease in loading capacity.

\subsubsection{Control Group: No Delamination with Embedded Arrestment Key}

Starting off with the investigation, a control group was used to provide comparable differences between the experimental groups and the control group. In this case, the test specimen was manufactured and a delamination was not introduced into the structure but the embedment of the arrestment key was investigated. The table below shows the comparable material behaviors between a test specimen that did not have any flaws or embedment of a key, denoted as ' 11 inches', and the 'No Delamination' configuration (ND).

Table 28: No Delamination Configuration

\begin{tabular}{|c|c|c|c|c|}
\hline Case & $\begin{array}{c}\text { Slenderness } \\
\text { Ratio }\end{array}$ & $\begin{array}{c}\text { Maximum Vertical } \\
\text { Extension }\end{array}$ & $\begin{array}{c}\text { Maximum } \\
\text { Load }\end{array}$ & $\begin{array}{c}\text { Maximum } \\
\text { Horizontal } \\
\text { Extension }\end{array}$ \\
\hline- & - & in & lbf & in \\
\hline 11 inches & 65.39673 & 0.11083 & 685.60279 & 0.42417 \\
\hline ND & 66.58938 & 0.09881 & 737.98110 & 0.49481 \\
\hline 0.5 ID & 66.06927 & 0.05020 & 479.36398 & 0.02274 \\
\hline 1.0 ID & 65.69982 & 0.03675 & 267.32638 & 0.00986 \\
\hline 1.5 ID & 66.21160 & 0.02963 & 158.26181 & 0.02399 \\
\hline 2.0 ID & 65.89214 & 0.04118 & 132.60860 & 0.04519 \\
\hline
\end{tabular}


Just by looking at the table, it details the ultimate loading capacity the specimen can take when being compressing. The vertical strain is slightly lower as well because the specimen is less flexible and has more stiffness. This extra loading capacity will be useful when dealing with delamination and when the structural integrity is jeopardized.

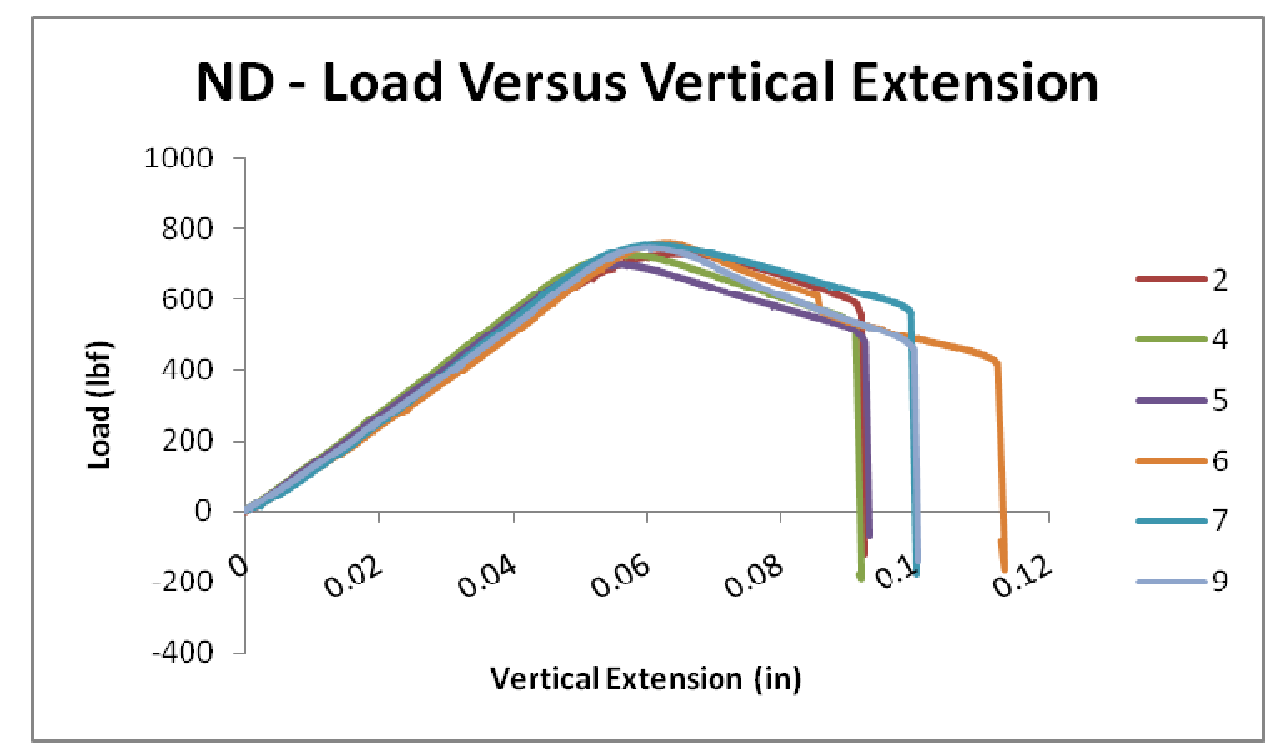

Figure 122: ND - Load Vs Vertical Extension

The load versus vertical extension detailed in Figure 122 shows how the geometric and material properties of the test specimens impact the loading over the vertical extension under loading. The rounded region around the ultimate is usually an indication that buckling has occurred. This leads to how the horizontal extension is related to the loading on the test specimen. Figure 123 shows quite a lot of horizontal displacement when the specimens are loaded. The test specimens would become unstable and rotate around its pinned-pinned boundaries. From both of the graphs so far, they show that the 'ND' test specimens are able to withstand much higher loading than the typical ' 11 inches' test specimens and maintain the majority its flexure and displacement. 


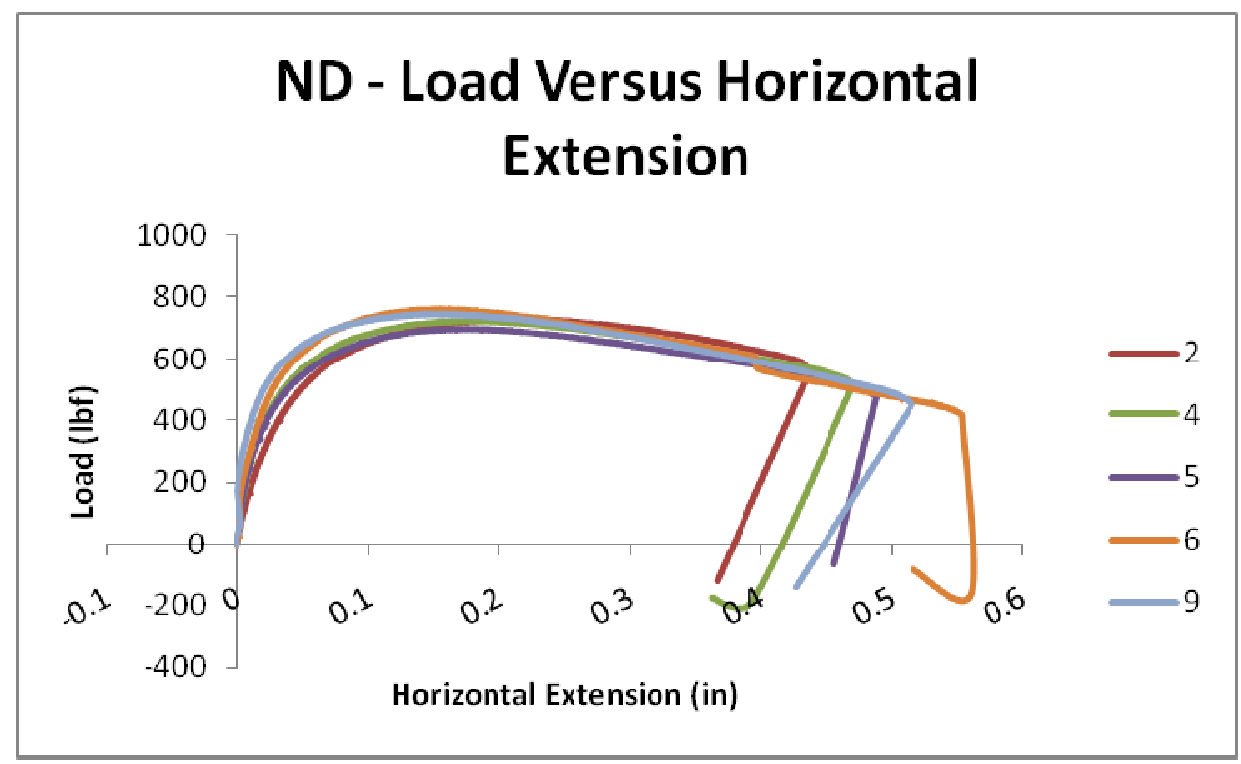

Figure 123: ND - Load Vs Vertical Extension

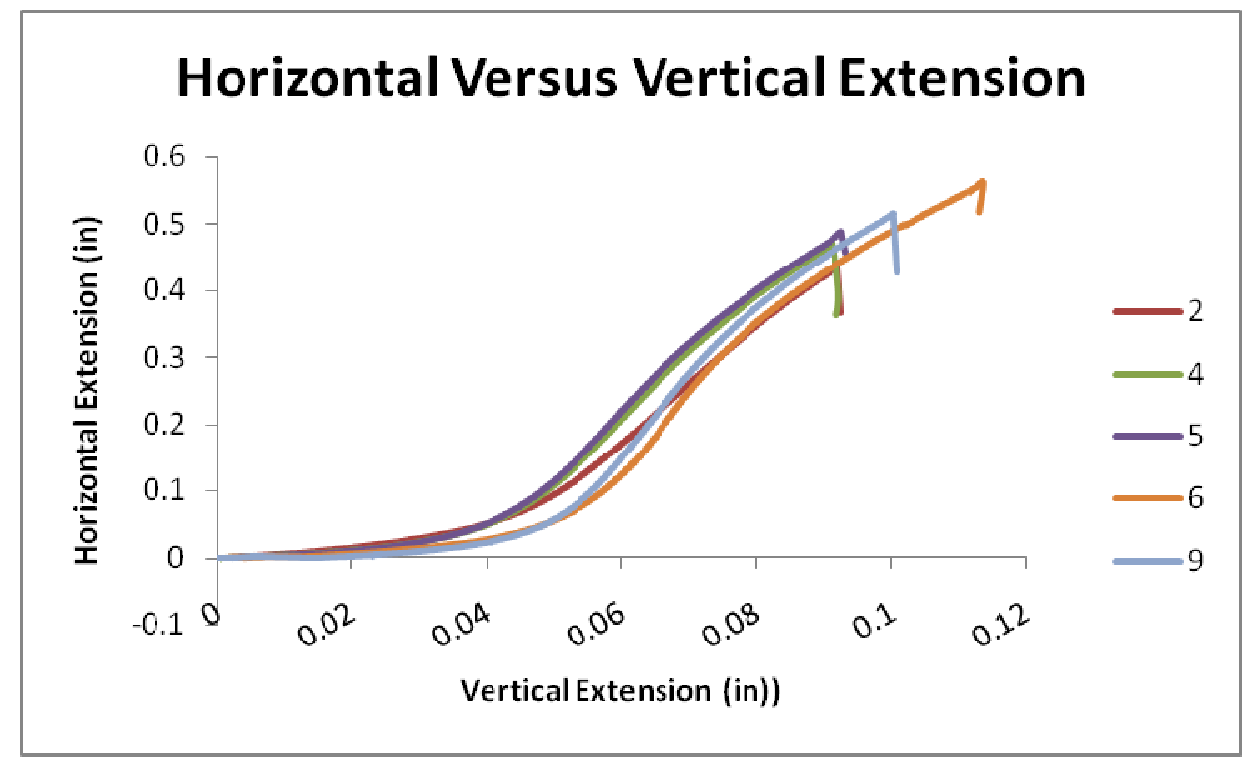

Figure 124: ND - Horizontal Vs Vertical Extension

Figure 124 is a combination of the horizontal extension to the vertical extension. It details how the specimens move when being loaded. The 'ND' test specimen begins to buckle when the load is first loaded then climbs until it fails. 


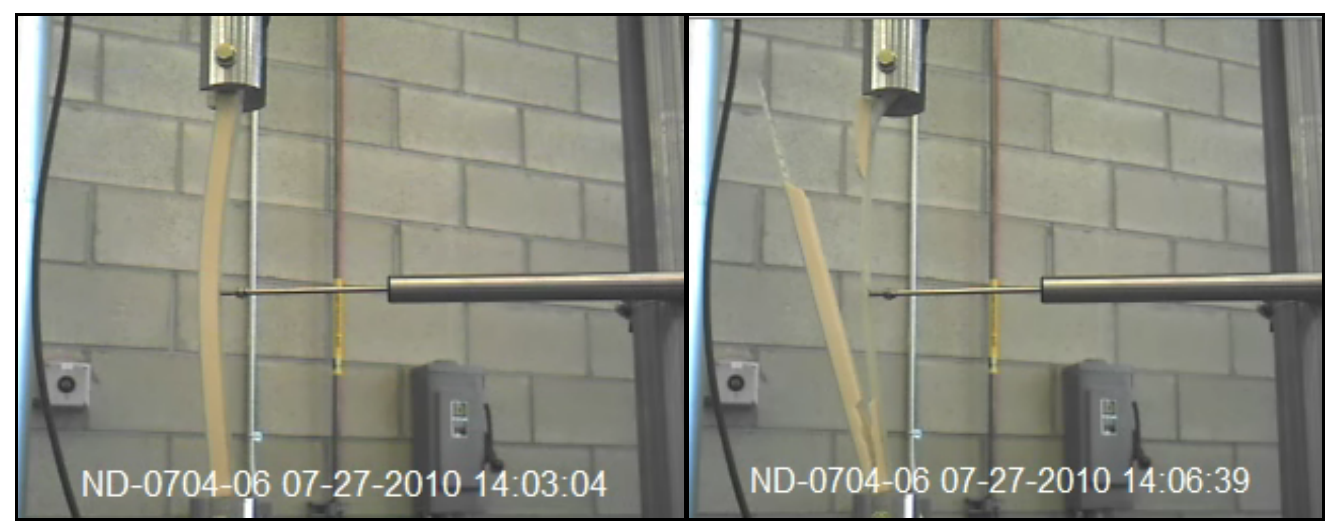

Figure 125: ND - Experimental Testing Before and After Failure

The experimental testing shows that the 'ND' configuration allows for buckling to still occur in the test specimen as seen in Figure 125 and the picture on the right shows the failure of the test specimen after it gave way.

\subsubsection{Experimental Groups}

The experimental groups look into the different variables that were considered such as a continuous delamination arrestment key placed in the horizontal or vertical directions, discontinuity in how the keys are placed, delamination where the skin is delaminated from the foam and key, and also where the skin and key is delaminated from the foam. Each of these experimental groups consists of 9 test specimens each.

\subsubsection{Experimental Group: Continuous Key in Vertical Direction with Skin Delamination}

For this section of the thesis project, the configuration for the test specimen consists of an embedded key placed in the vertical direction and a delamination region located at the center. The two delamination regions studied extend the width of the specimen and has either a 1-inch or 2inch length. The delamination area consists of a delamination between the key and the skin, but this does not separate the key from the foam core in the region where the delamination exists. The results from having the continuous key in the vertical direction did significantly improve the load capacity that the composite sandwich structure could take. This can be seen in Table 29. 
Table 29: SD1.0 and SD2.0 Results Comparison

\begin{tabular}{|c|c|c|c|c|}
\hline Case & $\begin{array}{c}\text { Slenderness } \\
\text { Ratio }\end{array}$ & $\begin{array}{c}\text { Maximum Vertical } \\
\text { Extension }\end{array}$ & $\begin{array}{c}\text { Maximum } \\
\text { Load }\end{array}$ & $\begin{array}{c}\text { Maximum } \\
\text { Horizontal } \\
\text { Extension }\end{array}$ \\
\hline- & - & in & lbf & in \\
\hline 11 inches & 65.39673 & 0.11083 & 685.60279 & 0.42417 \\
\hline ND & 66.58938 & 0.09881 & 737.98110 & 0.49481 \\
\hline 1.0 ID & 65.69982 & 0.03675 & 267.32638 & 0.00986 \\
\hline 2.0 ID & 65.89214 & 0.04118 & 132.60860 & 0.04519 \\
\hline SD1.0 & 65.63689 & 0.08530 & 604.48262 & 0.30740 \\
\hline SD2.0 & 64.88100 & 0.10069 & 680.74380 & 0.28825 \\
\hline
\end{tabular}

From the results, what was interesting was the loading capacity between the 'SD1.0' and 'SD2.0' data. 'SD1.0' is denoted for the 1-inch skin delamination test specimens with the vertical key and 'SD2.0' is for the 2-inch skin delamination specimens. The loading capacity for 'SD2.0' is a bit higher than 'SD1.0' and that is the interest of this discussion. The reason for this phenomenon is probably because the large skin delamination allows for the key to buckle with the rest of the structure a bit more. The key is buckling and is taking more loading under this condition before breaking. 'SD1.0' test specimens fail much earlier than the 'SD2.0' test specimens. The results do show that the 'ND' is still the leading and most preferable specimen for loading capacity, but 'SD1.0' and 'SD2.0' were able to maintain the majority of the loads and extension when the specimens were subjected to the in-plane loading.

\subsection{1-Inch Delamination}

From Figure 126, it is quite interesting to look at the initial load climbing before the load begins to drop. The graph differs quite a bit from the 'ND' graph in Figure 122 where the extension is slightly shorter. This means the test specimens fail a bit earlier than the 'ND' and '11 inches' specimens, but is still a significant improvement from the composite sandwich structures with just a delaminated region and no embedded key. 


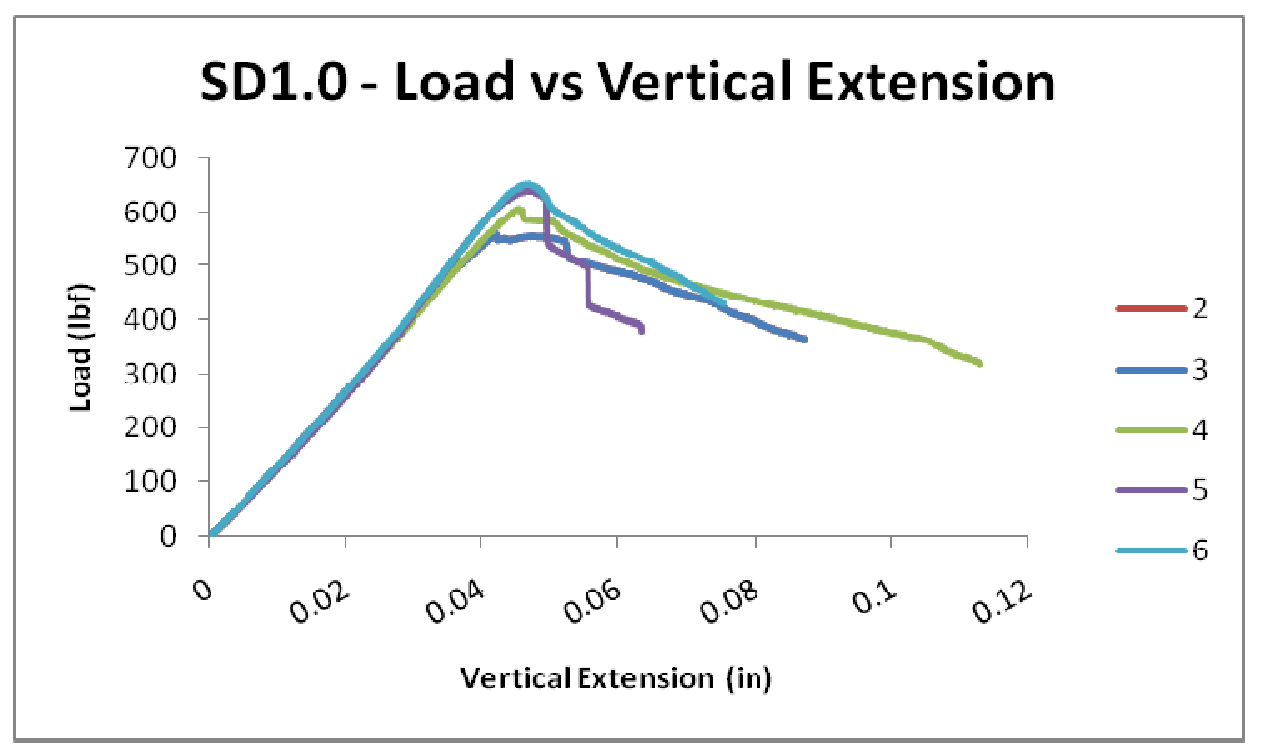

Figure 126: SD1.0 - Load Vs Vertical Extension

There are less horizontal displacement for the 'SD1.0' test specimens than the 'ND' and ' 11 inches' test specimens due to the embedded key. The embedded key increases the moment of area and the geometry change must be taken into consideration to better understand the upgraded structure. Figure 127 shows the load associated with the horizontal extension where the extensometer is placed on the delamination region side and Figure 128 shows the load associated with the horizontal extension where the extensometer is placed on the non-delamination side of the structure. Figure 127 and Figure 128 show similar trends because the delaminated region is quite small and the face sheet did not buckle as much. As discussed in the earlier section, limited resources were able to only provide one extensometer, which required several specimens to be dedicated to measuring each side of the test specimens. 


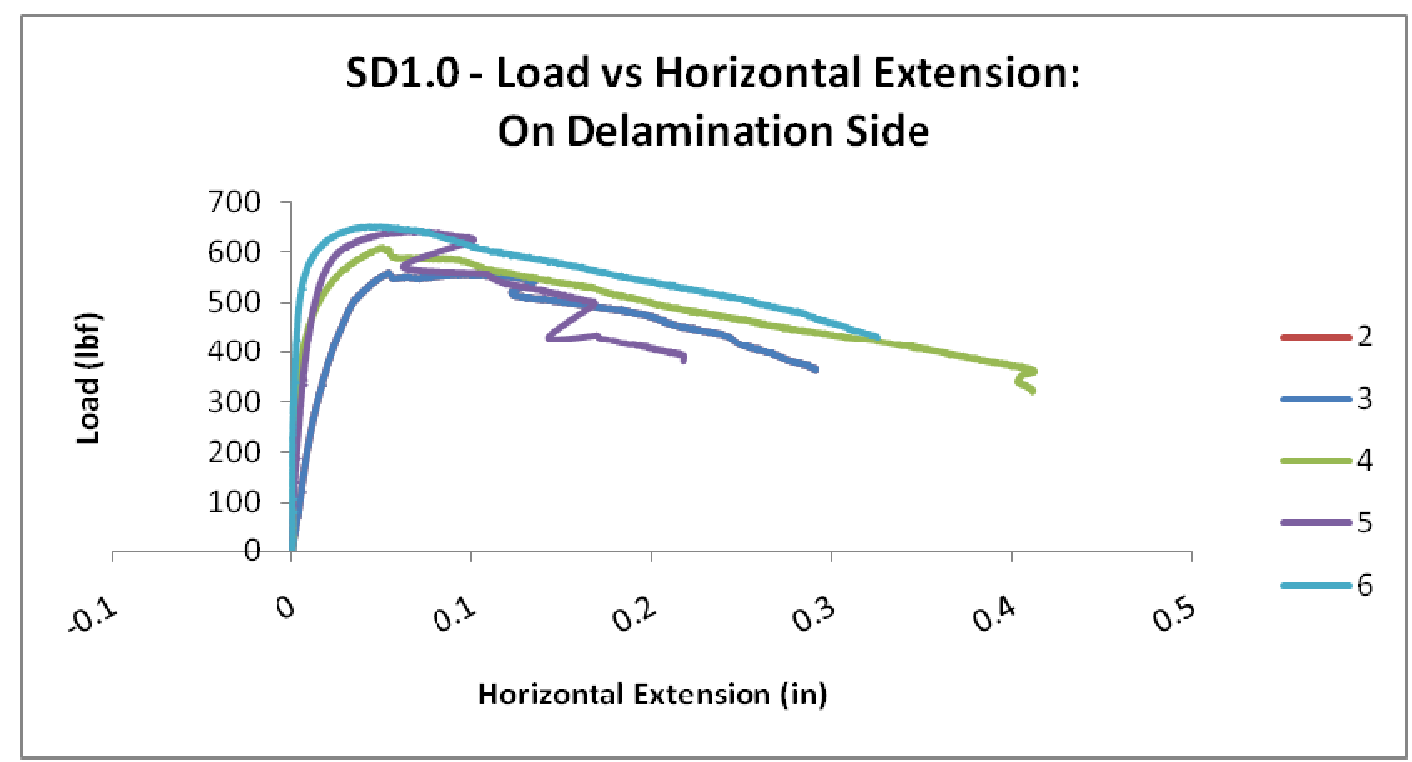

Figure 127: SD1.0 - Load Vs Horizontal Extension: On Delamination Side

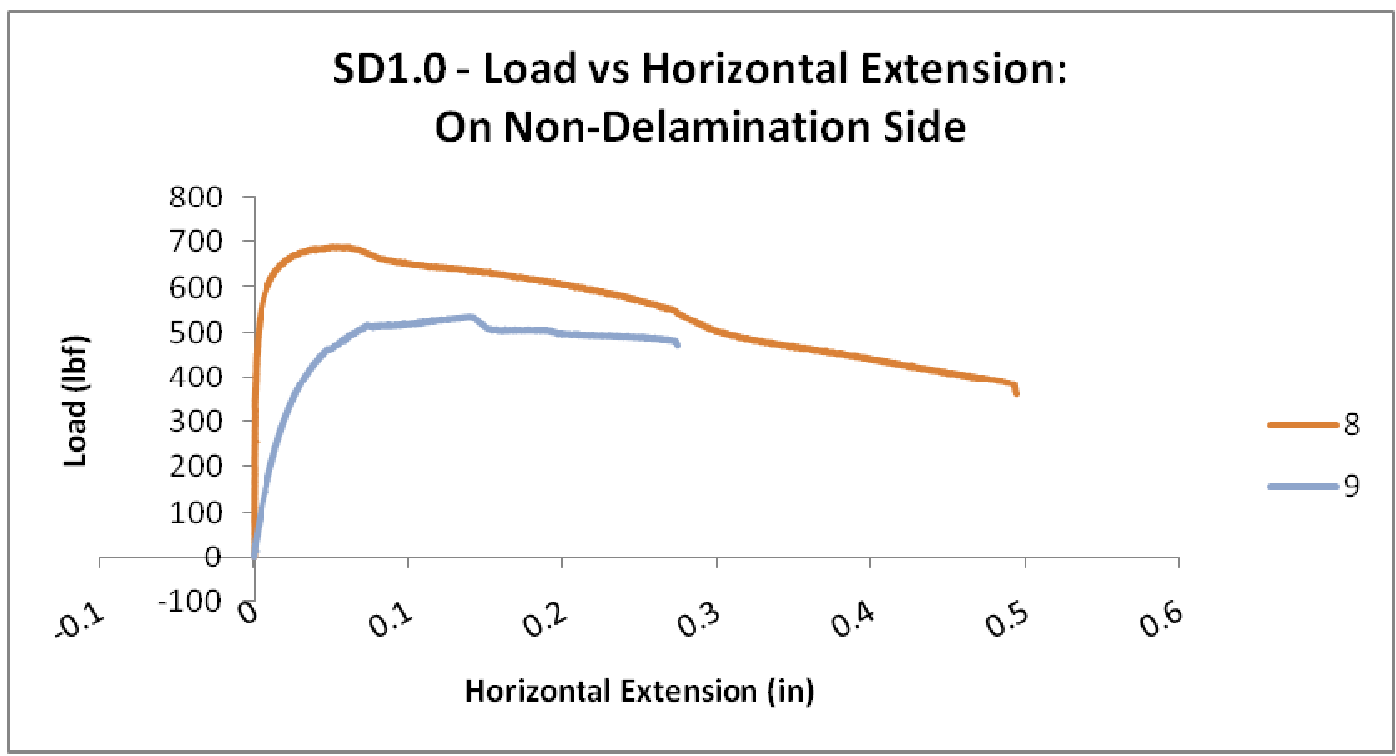

Figure 128: SD1.0 - Load Vs Horizontal Extension: On Non-Delamination Side

Figure 129 details the relationship between the horizontal extension and vertical

extension for the 1-inch delamination test specimens. The graph shows a small vertical extension before the horizontal extension begins to climb. Figure 130 details the same relationship but on the non-delamination side of the test specimens. Comparing the two graphs, the skin is showing more extension, which entails a higher buckling stress. The non-delamination side shows less extension and mostly due to the larger moment of inertia. 


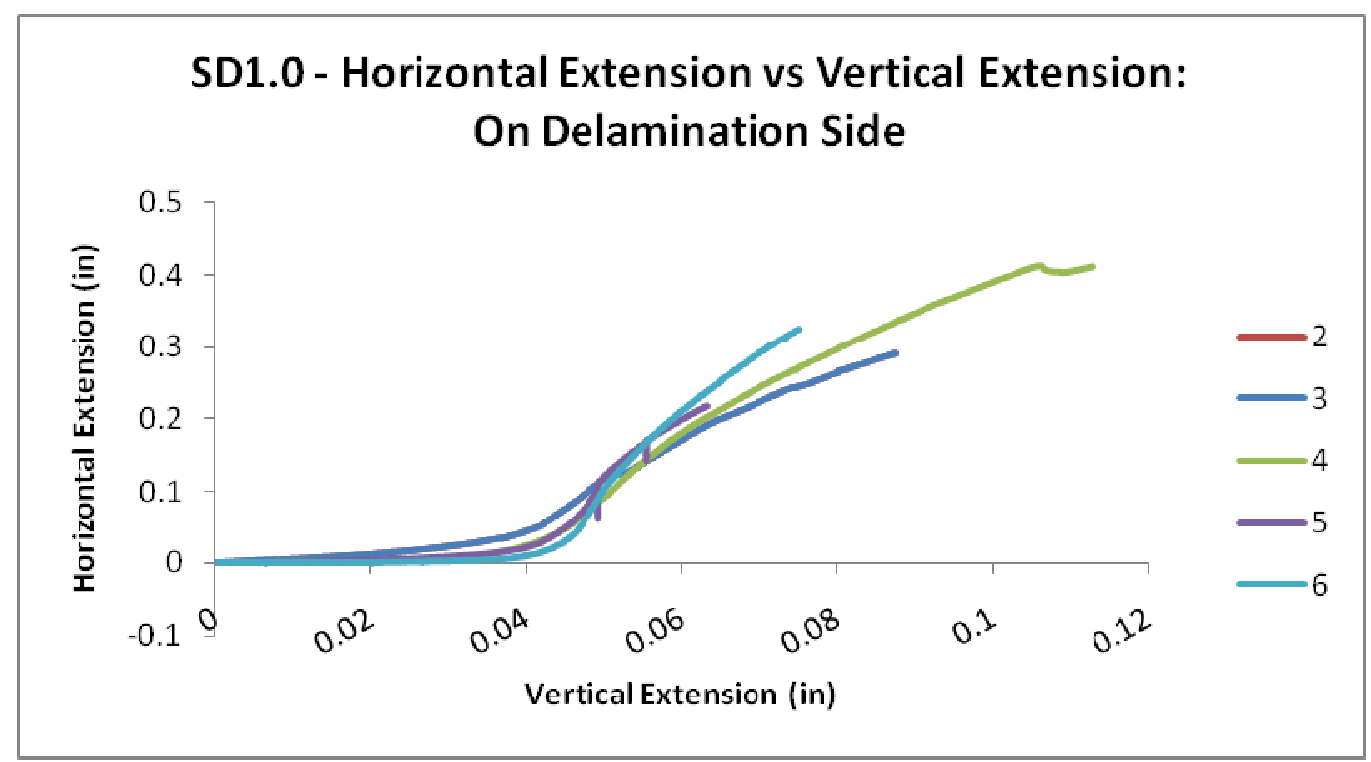

Figure 129: SD1.0 - Horizontal Extension Vs Vertical Extension: On Delamination Side

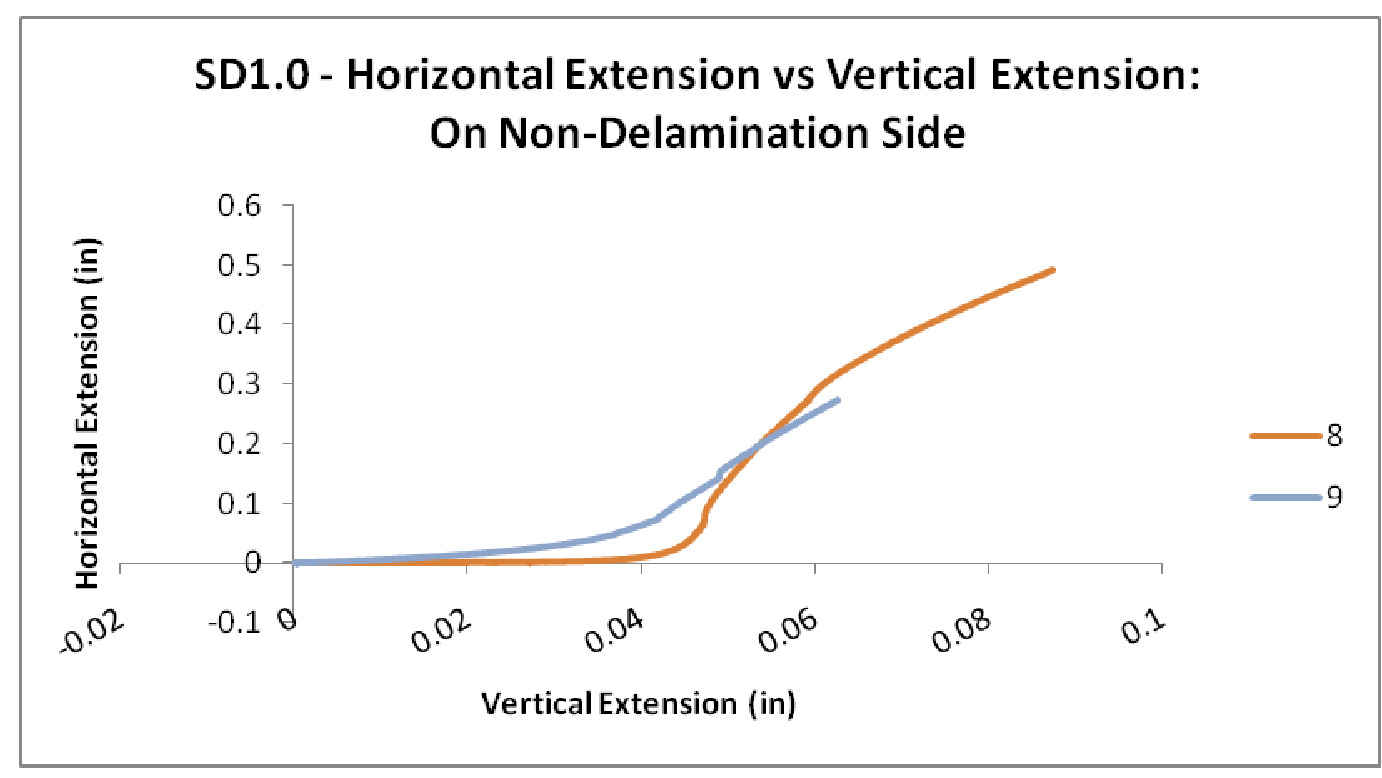

Figure 130: SD1.0 - Horizontal Extension Vs Vertical Extension: On Non-Delamination Side

Figure 131 shows the 'SD1.0' test specimen in action with the one extensometer placed on the delamination side. The test specimen shows the buckling effect of the structure and the face sheet in two different directions. 


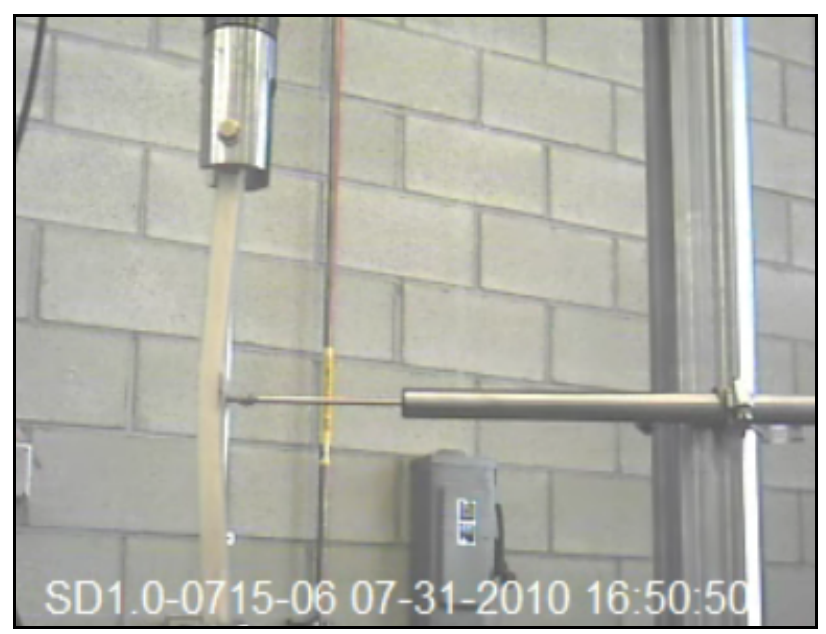

Figure 131: SD1.0 - Experimental Testing Before Failure

From what is seen in Figure 131, the graph shows that the 'SD1.0' configuration buckles the face sheet and is able to maintain its structural integrity for a longer time period. Figure 132 shows a structural failure when the load dropped as the delamination region begins to grow.

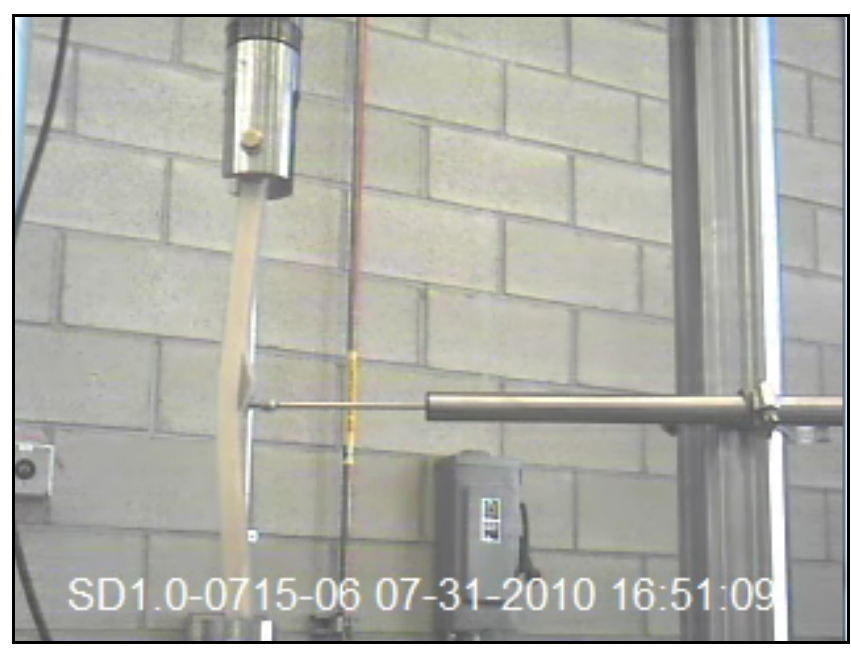

Figure 132: SD1.0 - Experimental Testing After Failure

\subsection{2-Inch Delamination}

Starting off with Figure 133, the graph is quite similar to the 'ND' graph for the load versus extension. The vertical extension and loading results are closer to the 'ND' results as well. What is quite interesting is how close this delaminated test specimen is compared to the original ' 11 inches' test specimens. The majority of the structural integrity is maintained and the key is great at transferring the load for this configuration with the skin being delaminated near the 
center. Note that the test specimens only had the extensometer placed on the delamination side of the structure.

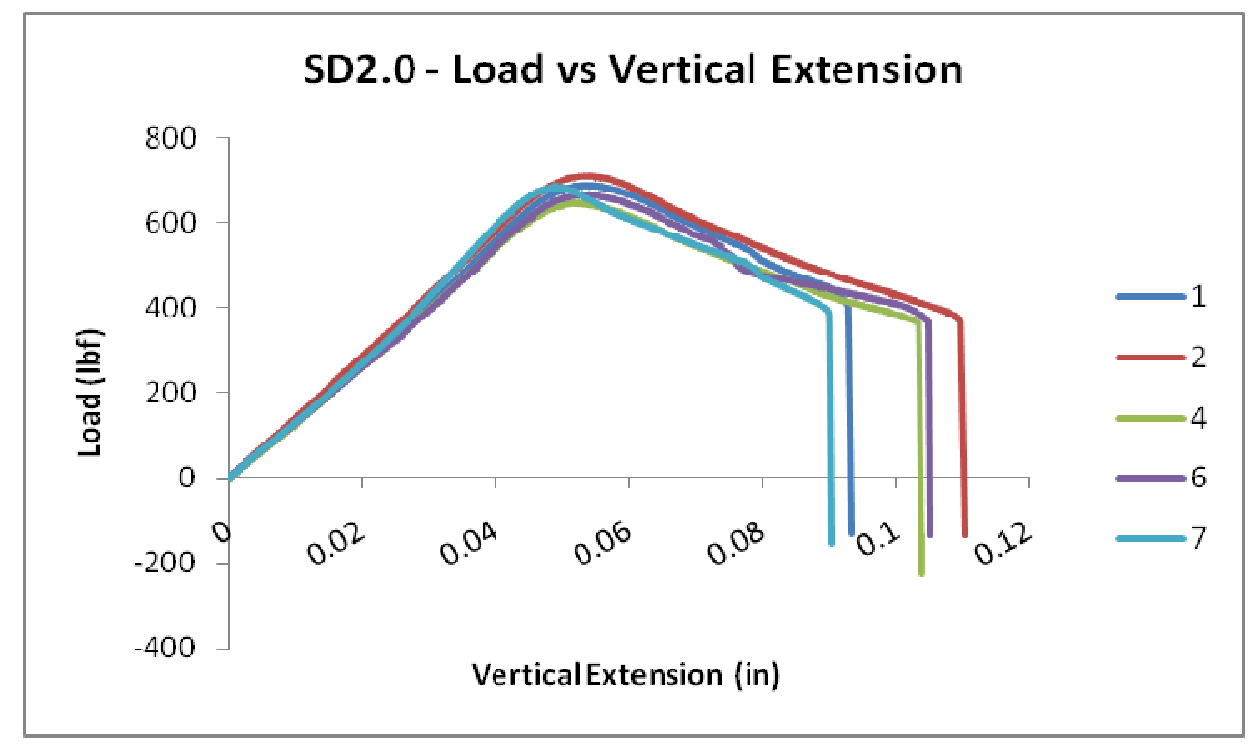

Figure 133: SD2.0 - Load Vs Vertical Extension

It is a bit different for Figure 134 though, the load versus the horizontal extension looks quite exotic in its trend but it is explainable. Starting from the origin of the graph, the curves progressively shows the buckling of the composite structure itself until the facesheet begins to buckle in the opposite direction to the rest of the structure. The extension differences between the two directions goes up to where the curves hit the $y$-axis and the facesheet extension data takes over. The horizontal displacement increases as the loading decreases. 


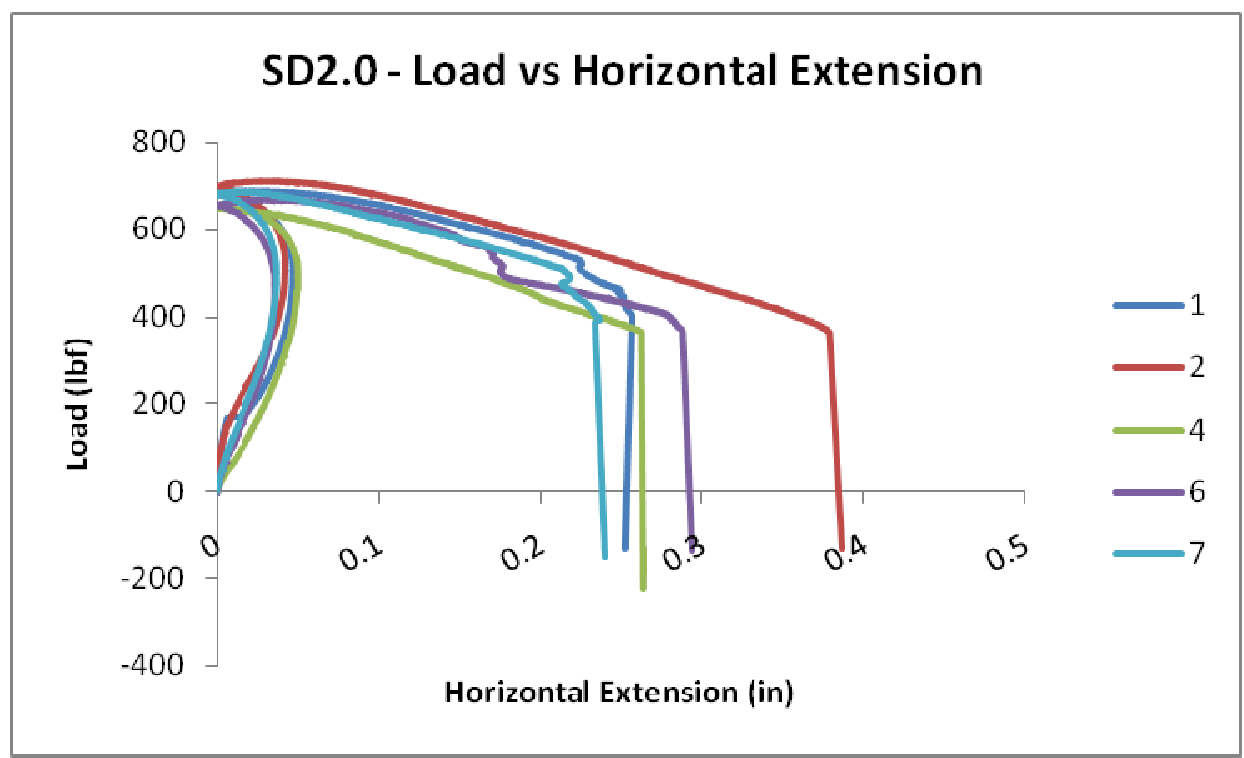

Figure 134: SD2.0 - Load Vs Horizontal Extension

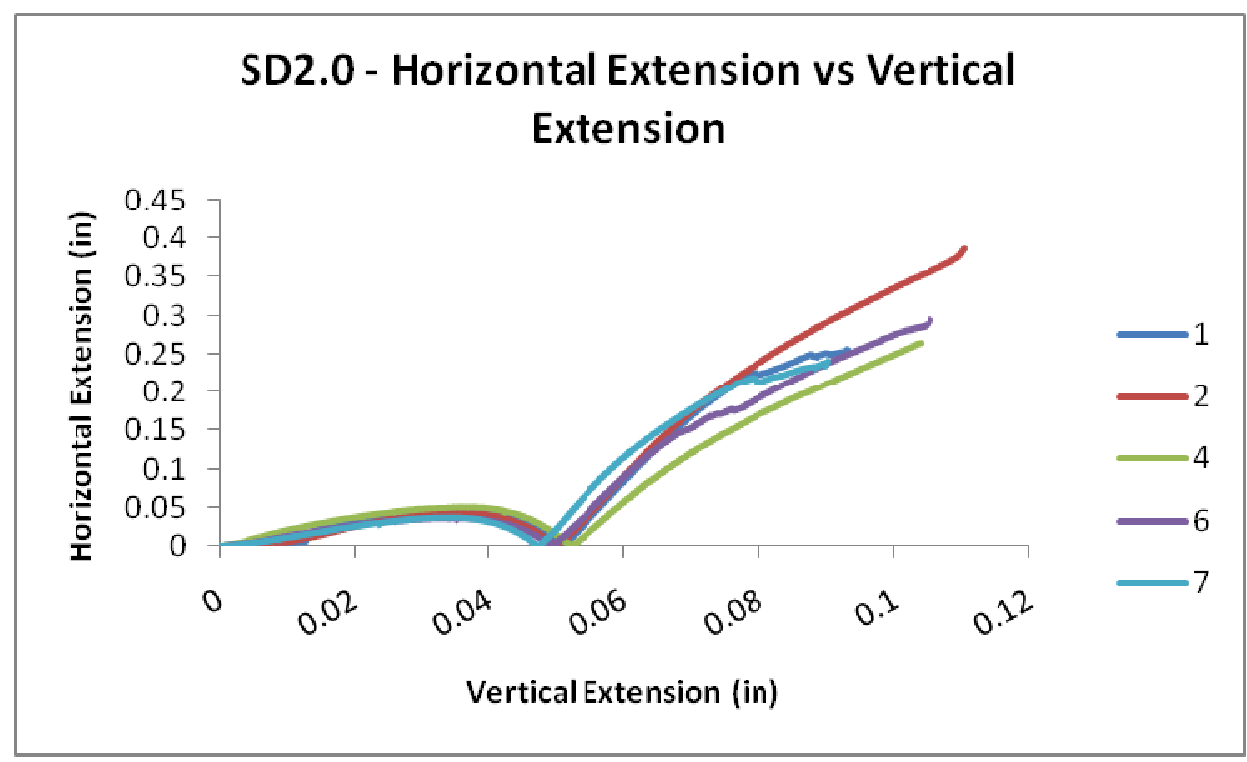

Figure 135: SD2.0 - Horizontal Extension Vs Vertical Extension

Figure 135 differs quite a bit from what was seen for 'SD1.0' test specimens. There is an initial buckling of the overall structure before the delamination begins to give way to the buckling of the face sheet. This keeps the structure intact longer and the delamination displacement smaller over a longer time period. 


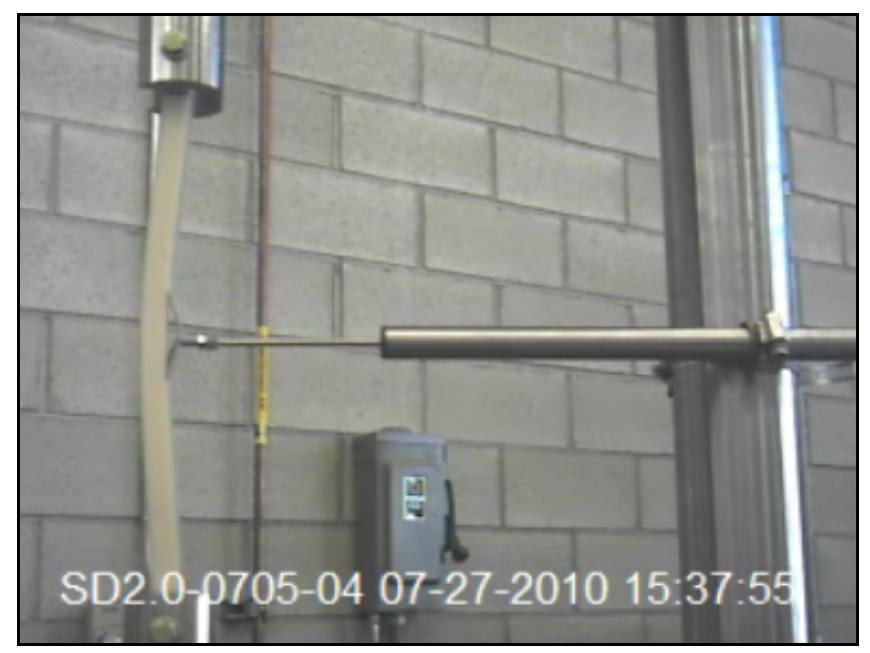

Figure 136: SD2.0 - Experimental Testing Before Failure

Similar to what was seen in the previous subsection, the experimental testing shows buckling of the structure and the face sheet in two different directions. In Figure 136, the buckled face sheet is more visible due to the delamination size. That is also true for the failure seen in Figure 137 where the delamination propagated upward.

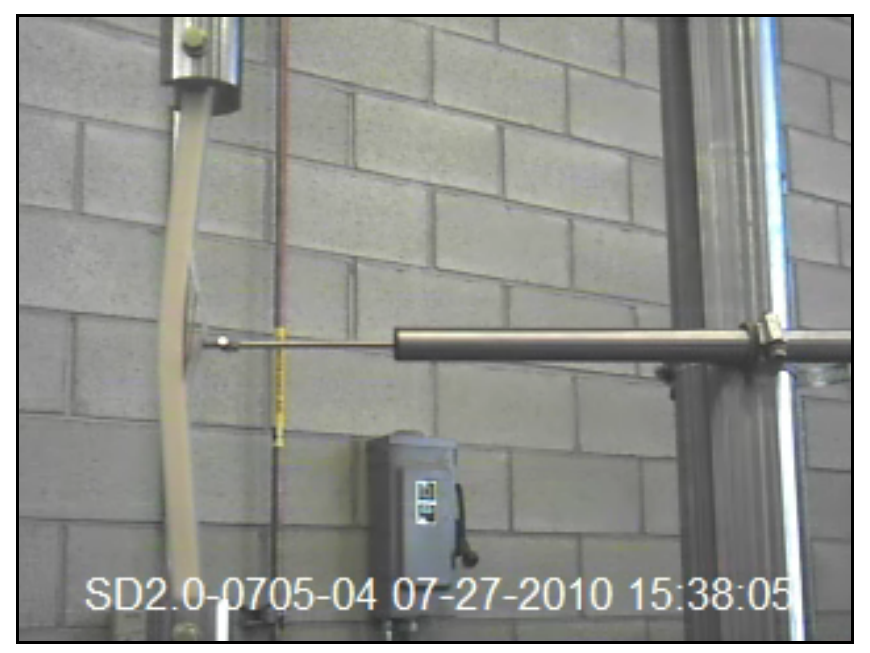

\section{Figure 137: SD2.0 - Experimental Testing After Failure}

\subsubsection{Experimental Group: Continuous Key in Vertical Direction with Key Delamination}

With the continuous study of how the continuous key in the vertical direction will impact the configuration, another tweak was investigated. That is where the arrestment key is delaminated from the foam core but maintains its bonding to the face sheet. Even with the key 
delaminated from the foam core; the test specimens were able to maintain much of their structural integrity. What is quite interesting is that the delamination shows signs of separation per 1-inch configuration while the 2-inch delamination configuration shows slight delamination between foam and the skin-key combo. The table with the results is displayed in Table 30.

\section{Table 30: KD Configuration Comparison}

\begin{tabular}{|c|c|c|c|c|}
\hline Case & $\begin{array}{c}\text { Slenderness } \\
\text { Ratio }\end{array}$ & $\begin{array}{c}\text { Maximum Vertical } \\
\text { Extension }\end{array}$ & $\begin{array}{c}\text { Maximum } \\
\text { Load }\end{array}$ & $\begin{array}{c}\text { Maximum } \\
\text { Horizontal } \\
\text { Extension }\end{array}$ \\
\hline- & - & in & lbf & in \\
\hline 11 inches & 65.39673 & 0.11083 & 685.60279 & 0.42417 \\
\hline ND & 66.58938 & 0.09881 & 737.98110 & 0.49481 \\
\hline 1.0 ID & 65.69982 & 0.03675 & 267.32638 & 0.00986 \\
\hline 2.0 ID & 65.89214 & 0.04118 & 132.60860 & 0.04519 \\
\hline KD1.0 & 65.12548 & 0.08611 & 864.98802 & 0.56654 \\
\hline KD2.0 & 66.27747 & 0.06617 & 746.09667 & 0.27680 \\
\hline
\end{tabular}

\subsection{1-Inch Delamination}

As discussed, the 'KD1.0' configuration resembles the 'ND' configuration except there is a 1-inch delamination. The 1-inch delamination did not even show up in any of the specimens, rather all the test specimens buckled similar to the 'ND' configuration. The 'KD1.0' has a higher loading capacity than the 'ND' tests specimens because there may have been a bit of a higher epoxy concentration where the non-porous material was placed during the manufacturing process. This is only a slight increase in loading capacity. Figure 138 even shows the same trend as 'ND' configuration. 


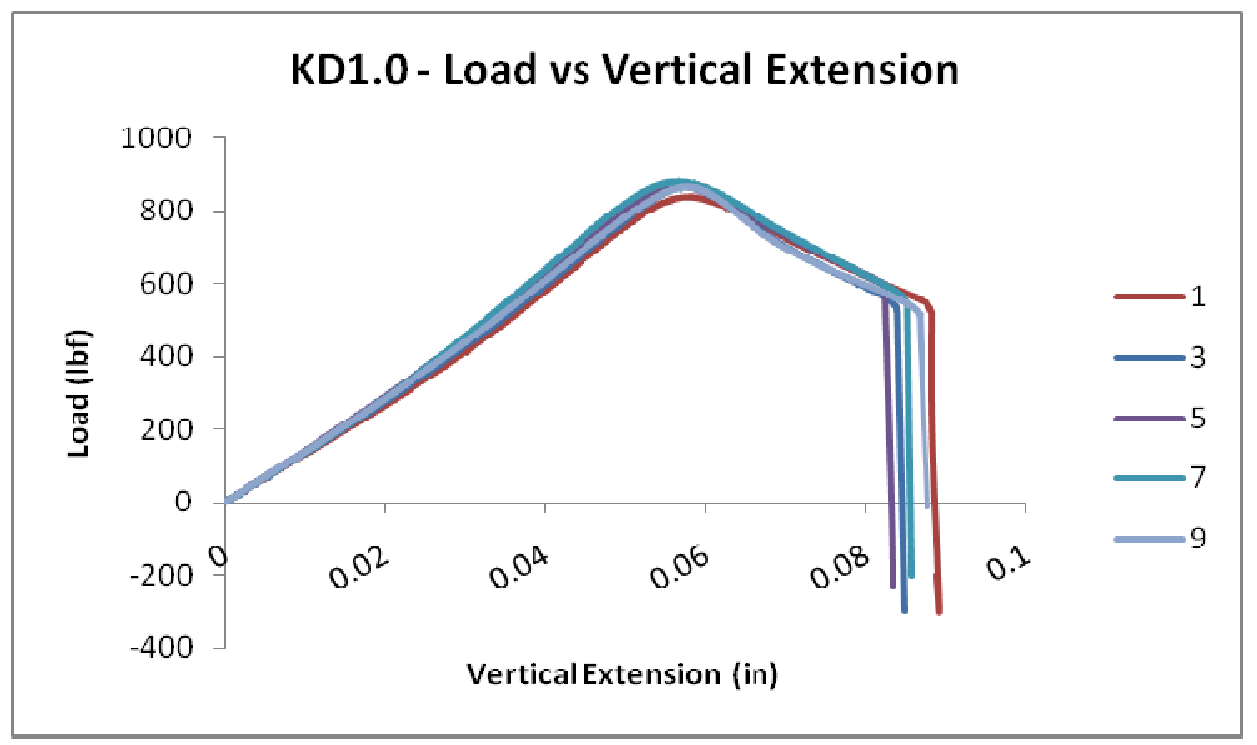

Figure 138: KD1.0 - Load Vs Vertical Extension

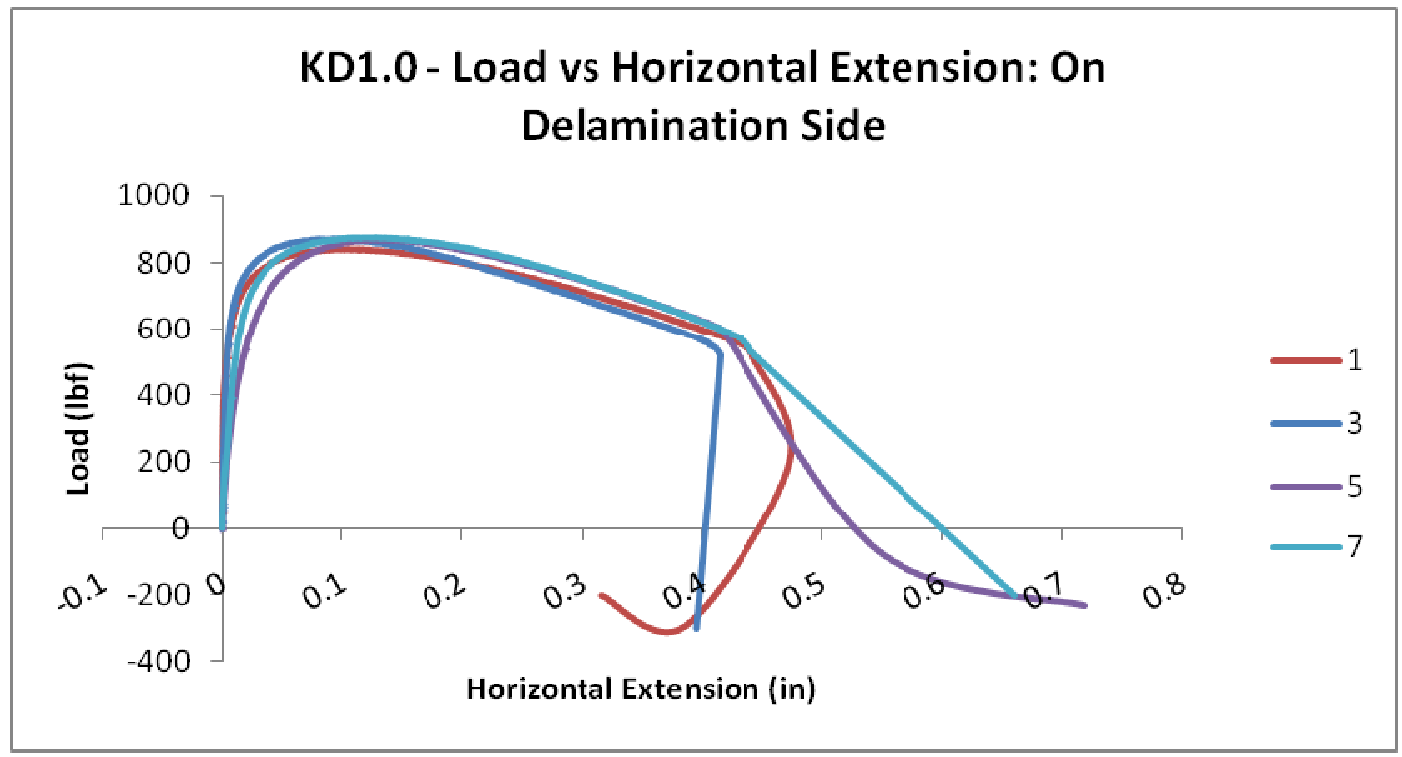

Figure 139: KD1.0 - Load Vs Horizontal Extension: On Delamination Side

Similarly, the horizontal extension did not differ from the 'ND' configuration as well.

This is seen in Figure 139 and Figure 140. Between the two graphs, the extensometer was placed on the two different sides to see if anything differs but the data did not differ at all. 


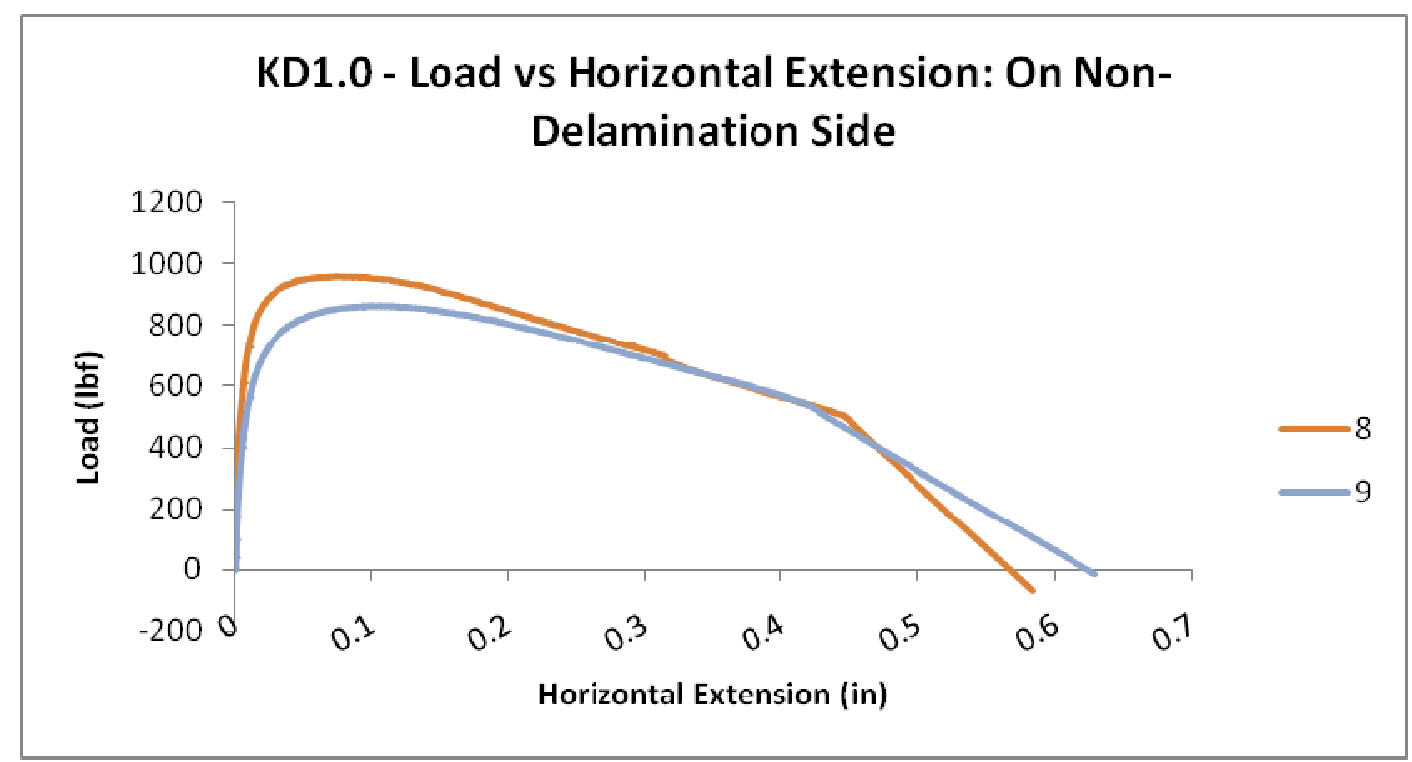

Figure 140: KD1.0 - Load Vs Horizontal Extension: On Non-Delamination Side

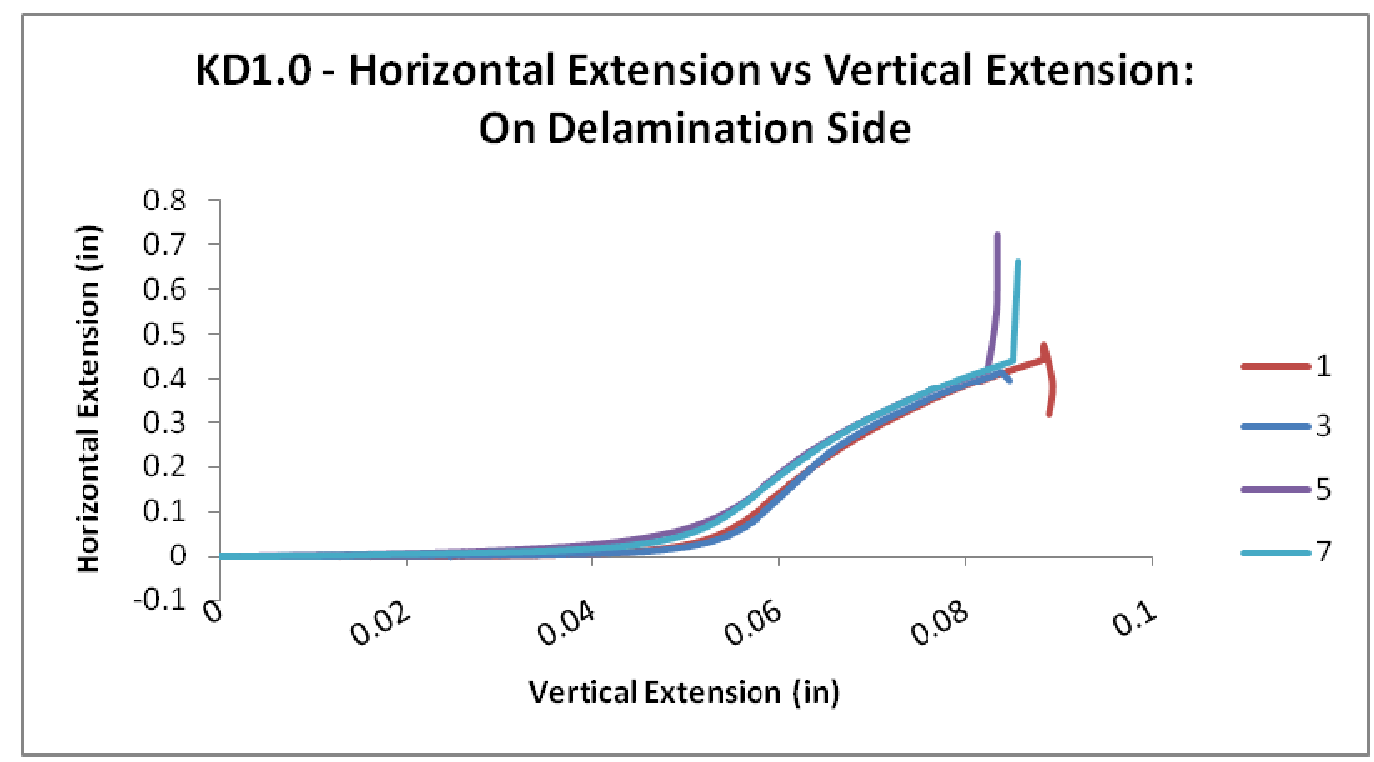

Figure 141: KD1.0 - Horizontal Extension Vs Vertical Extension: On Delamination Side

Figure 141 and Figure 142 do not show any differences either. The trends seen in both of these do not differ from what is seen in the 'ND' configuration. 


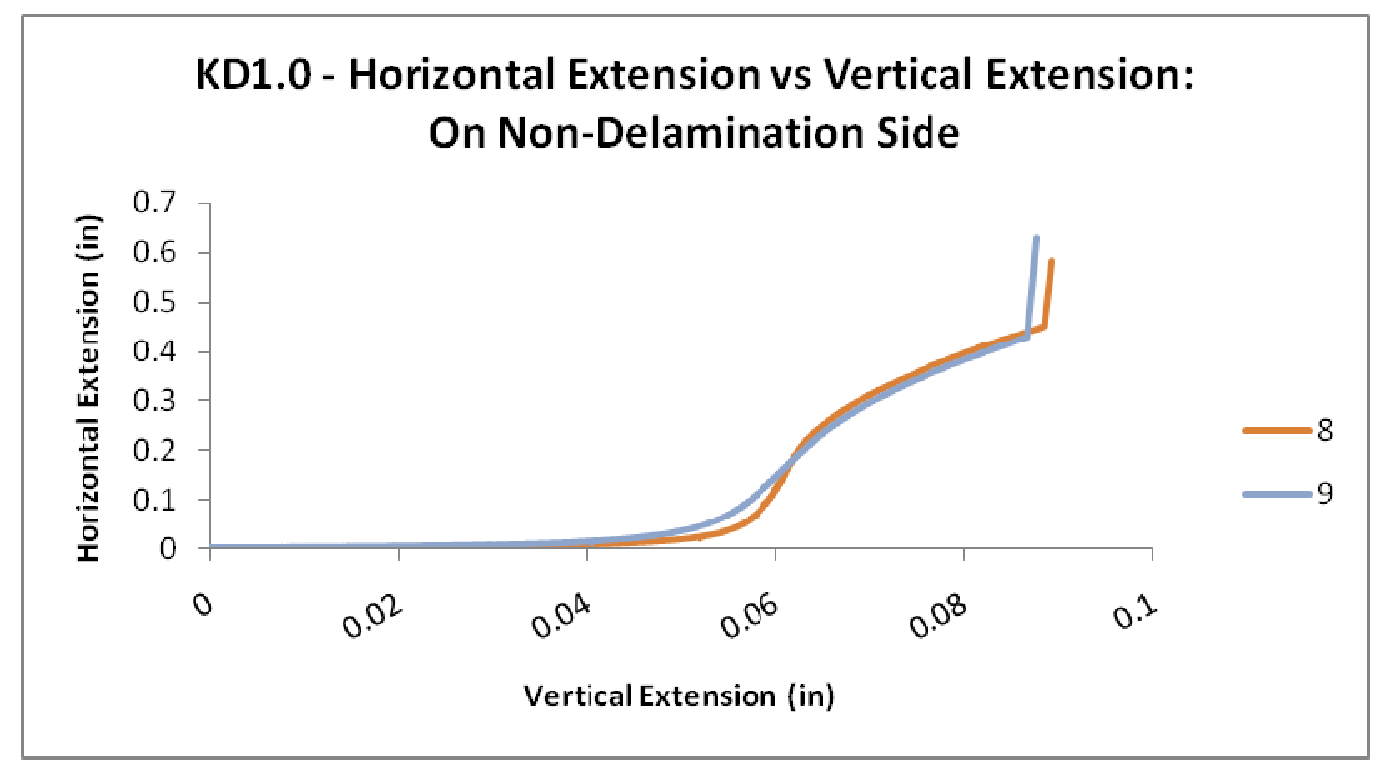

Figure 142: KD1.0 - Horizontal Extension Vs Vertical Extension: On Non-Delamination Side

From Figure 143, it can be seen how the 'KD1.0' buckles and is relatively the same as the 'ND' configuration. From Figure 144, the failure mode is a bit different. The difference is the fracture where the initial delamination is, but the crack propagation along one side of the test specimen is also found on the 'ND' configuration. This 'KD1.0' configuration is able to arrest the delamination and maintain the structural integrity.

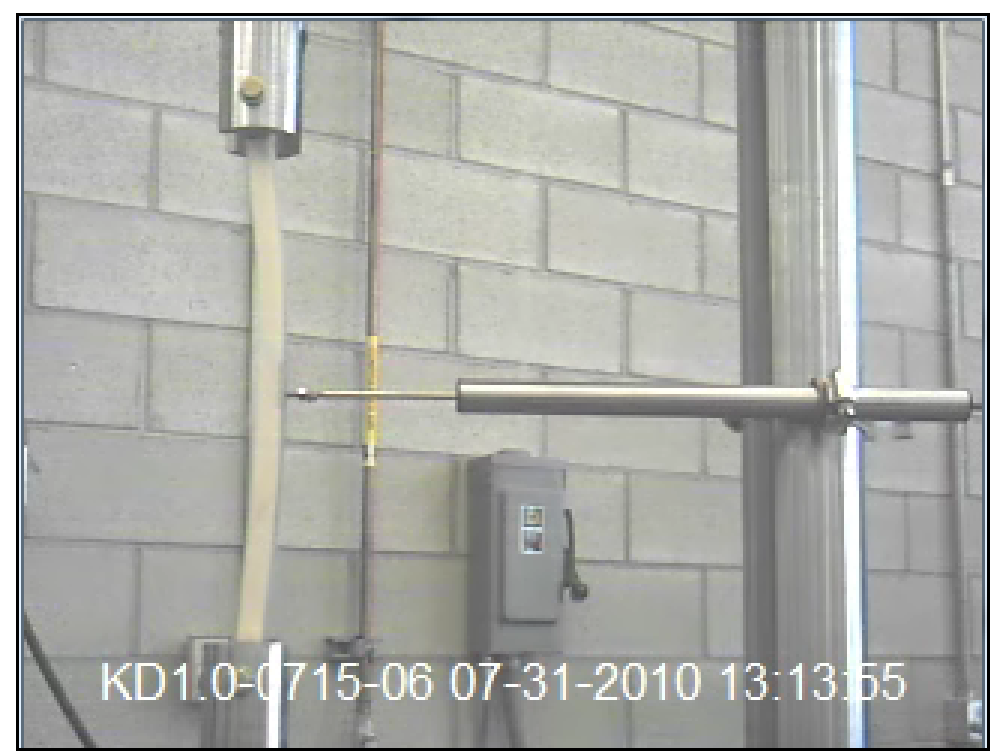

Figure 143: KD1.0 - Experiment Testing Before Failure 


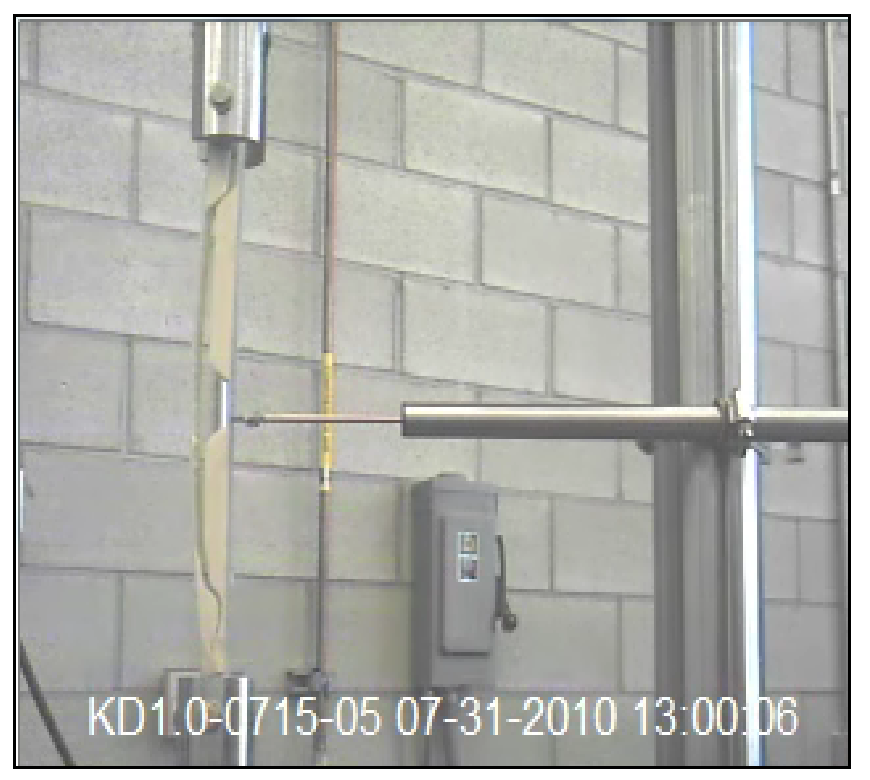

Figure 144: KD1.0 - Experimental Testing After Failure

\subsection{2-Inch Delamination}

Unlike the 'KD1.0' configuration, the 'KD2.0' configuration deviates quite a bit. The failure is much earlier and at a shorter vertical extension. It can be seen from Figure 145 that the loading capacity is maintained, but the test specimens fail a bit earlier.

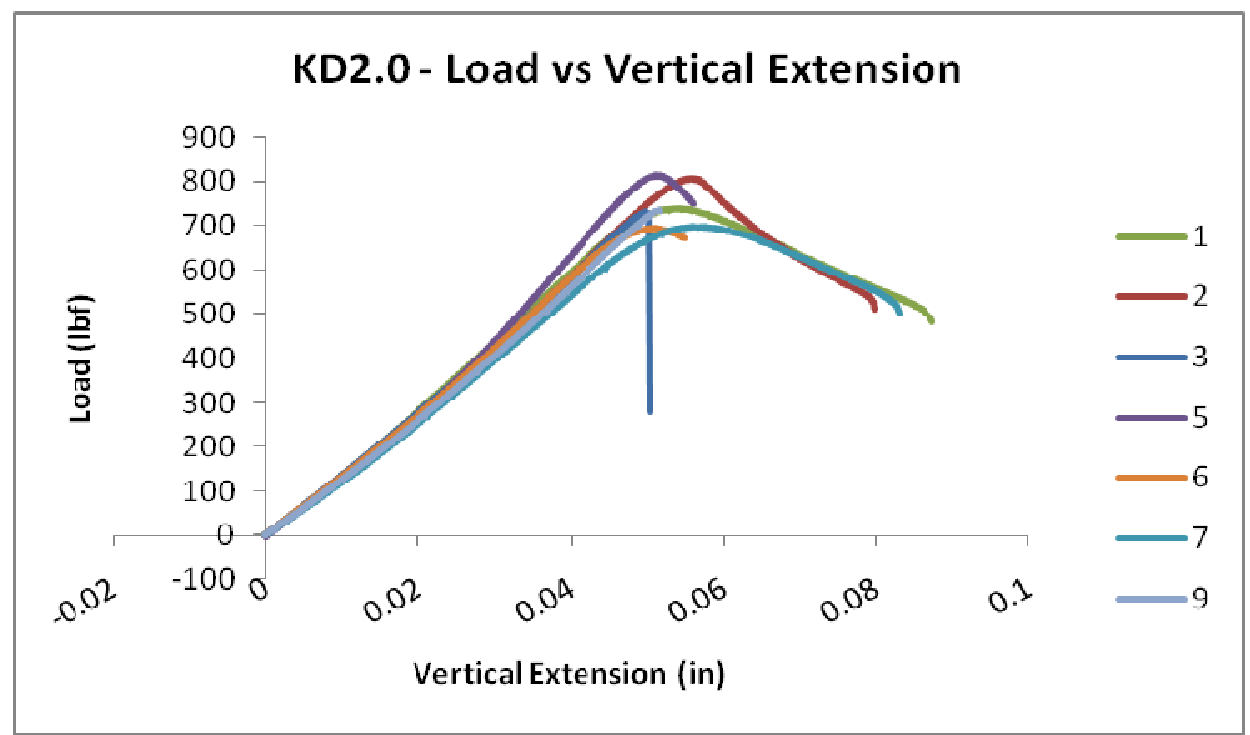

Figure 145: KD2.0 - Load Vs Vertical Extension 


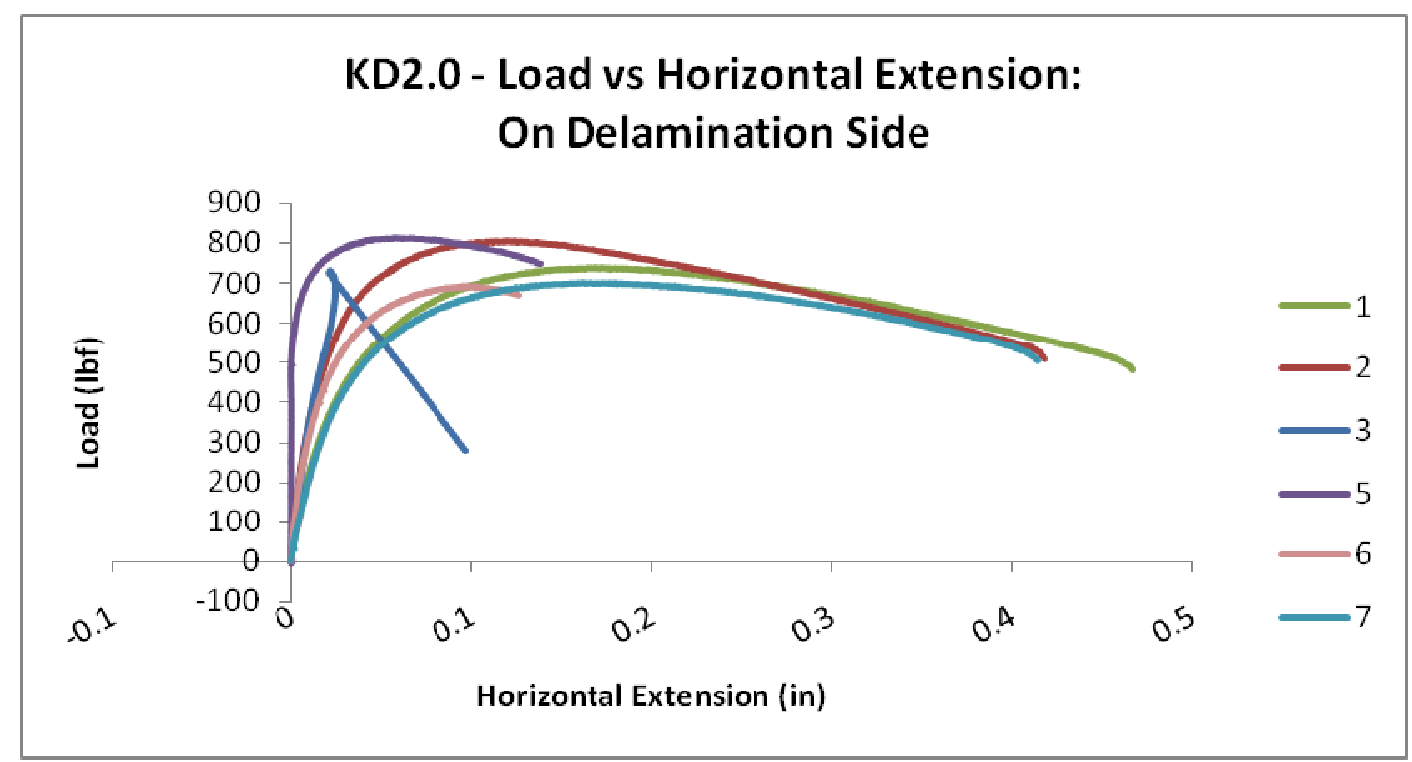

Figure 146: KD2.0 - Load Vs Horizontal Extension: On Delamination Side

The 'KD2.0' configuration does show a slight delamination of the key with the face sheet. This is why the trends found in Figure 146 and Figure 147 have a higher loading per horizontal extension until the buckling gives way under the vertical displacement. The difference between Figure 146 and Figure 147 is where the extensometer is placed. The non-delamination side differs in how it displaces than the delamination side. The difference is due to the geometry of the structure versus the skin-key combo.

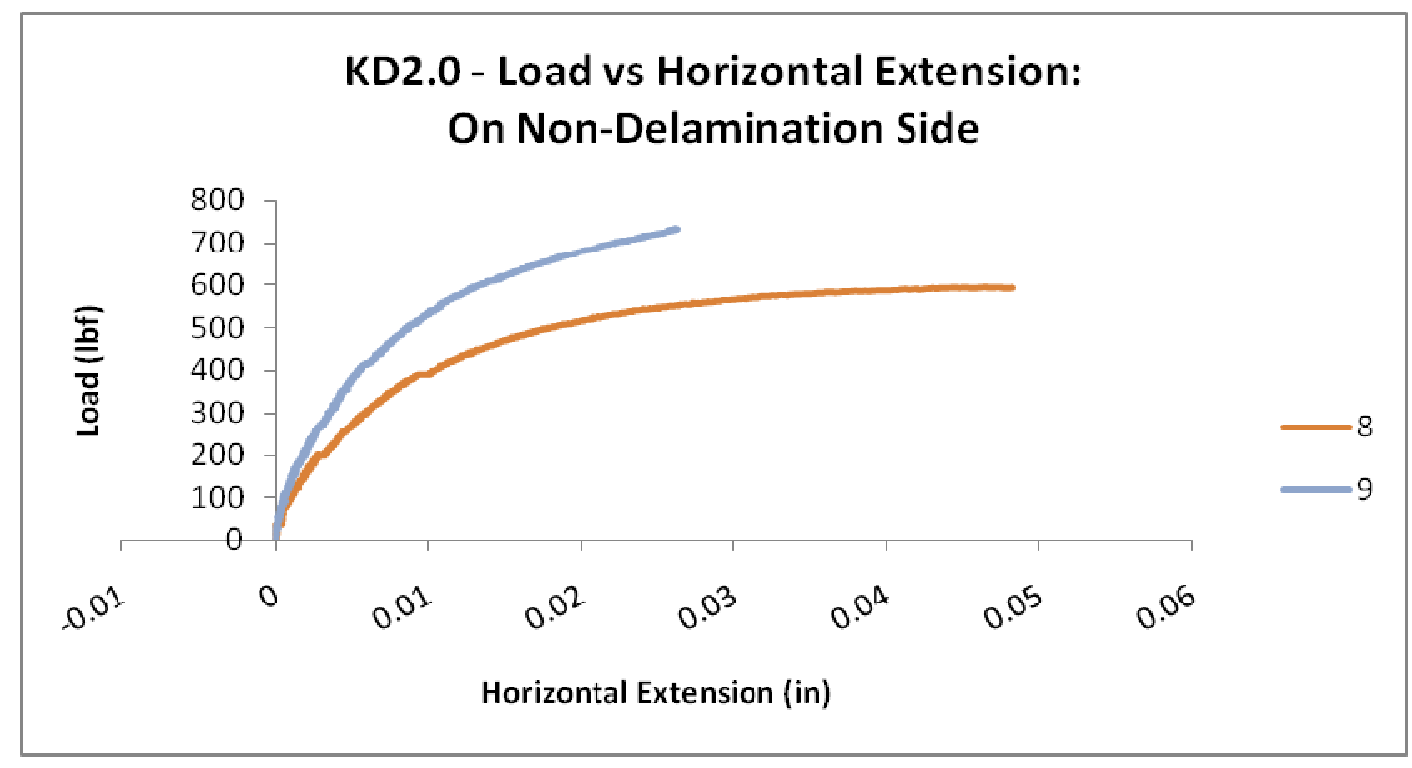

Figure 147: KD2.0 - Load Vs Horizontal Extension: On Non-Delamination Side 


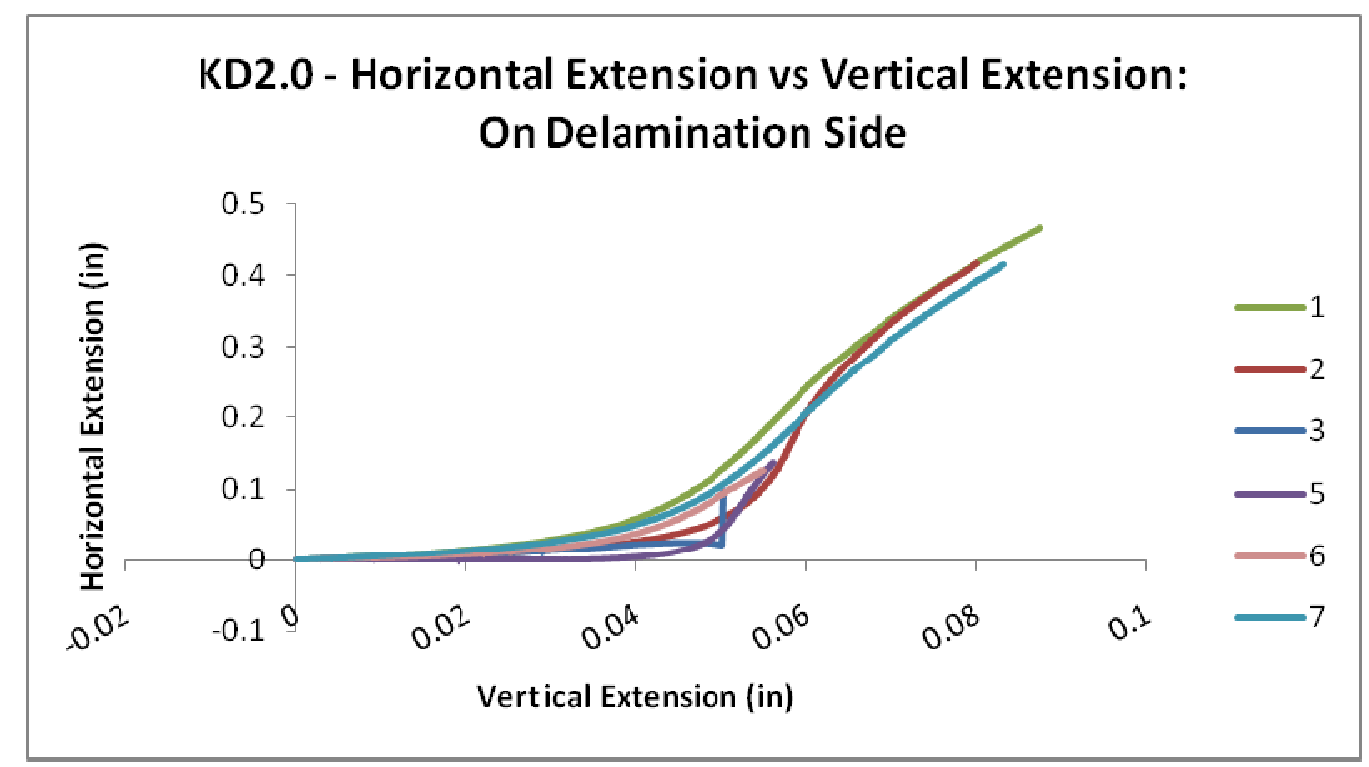

Figure 148: KD2.0 - Horizontal Extension Vs Vertical Extension: On Delamination Side

Figure 148 is quite similar to what is seen for 'KD1.0' configuration except at a shorter horizontal extension. The shorter horizontal extension is due to the stiffness of the structure because the moment of area is the combination of the key and the skin. This differs though for Figure 149 because the extensometer is placed on the non-delamination side of the test specimen. The data shows that this side of the test specimen can continue to displace even more before failure, but the delamination side gave way first.

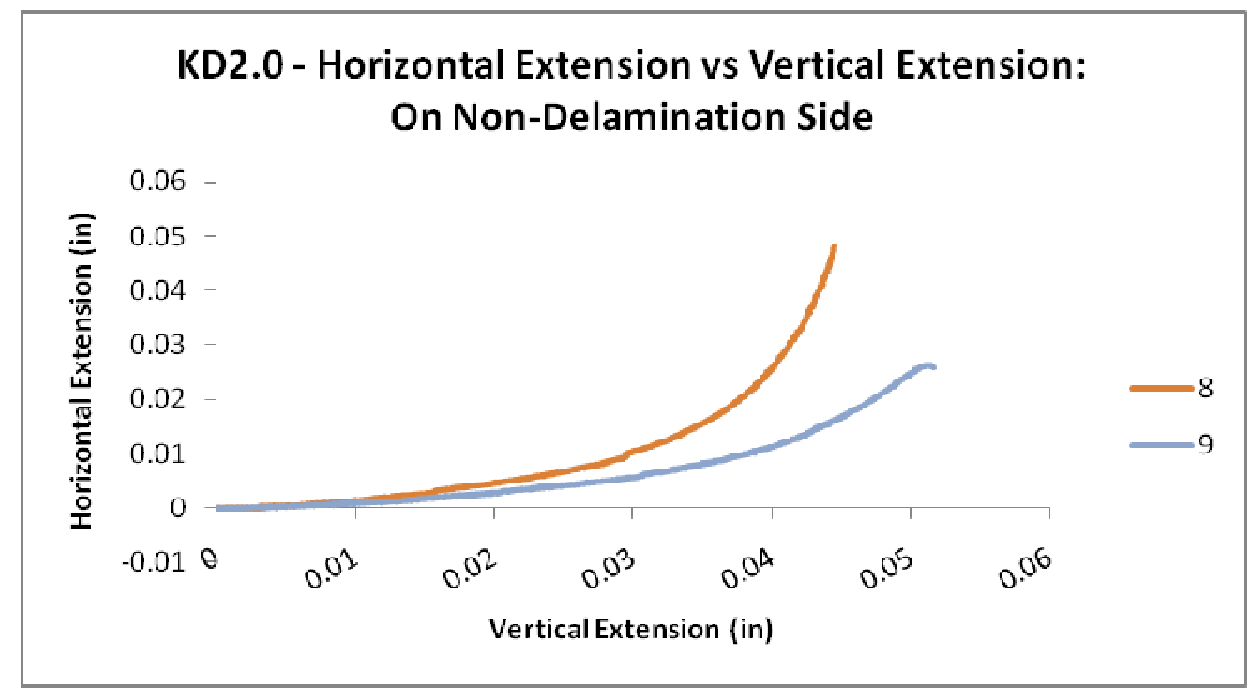

Figure 149: KD2.0 - Horizontal Extension Vs Vertical Extension: On Non-Delamination Side 


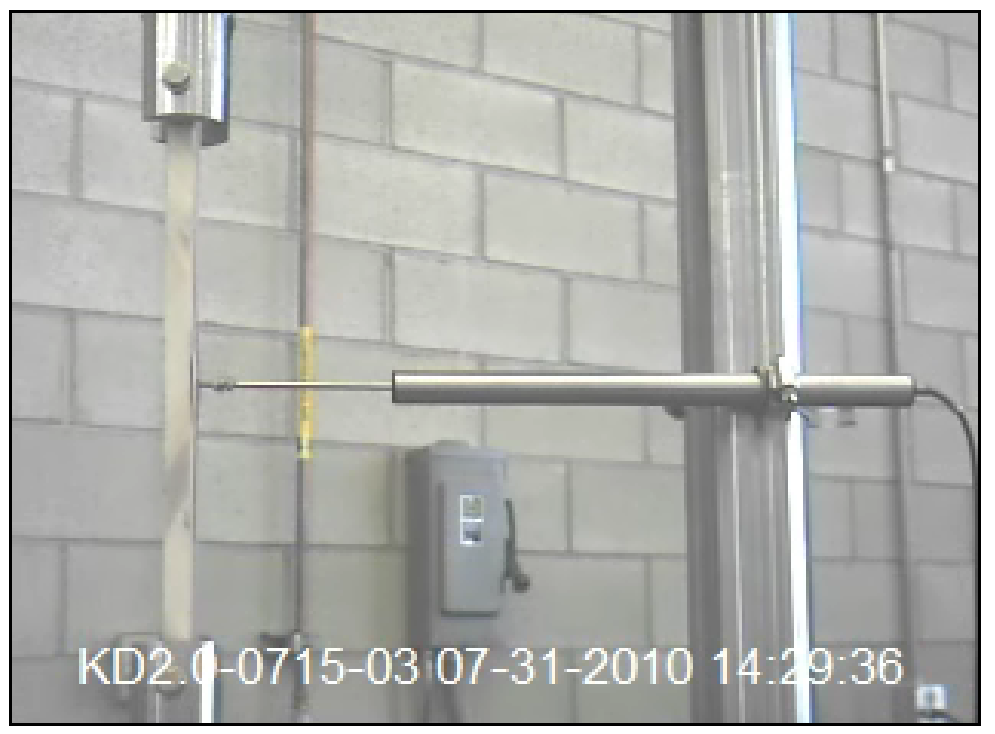

Figure 150: KD2.0 - Experimental Testing Before Failure

Figure 150 shows a visible horizontal displacement of delamination of the skin-key combo. The delamination is much smaller than many of the other configurations, which means the load is transferred through the moment of area of the skin-key combo much better than having the skin alone. As Figure 151, the entire initial delaminated side separated from the test specimen.

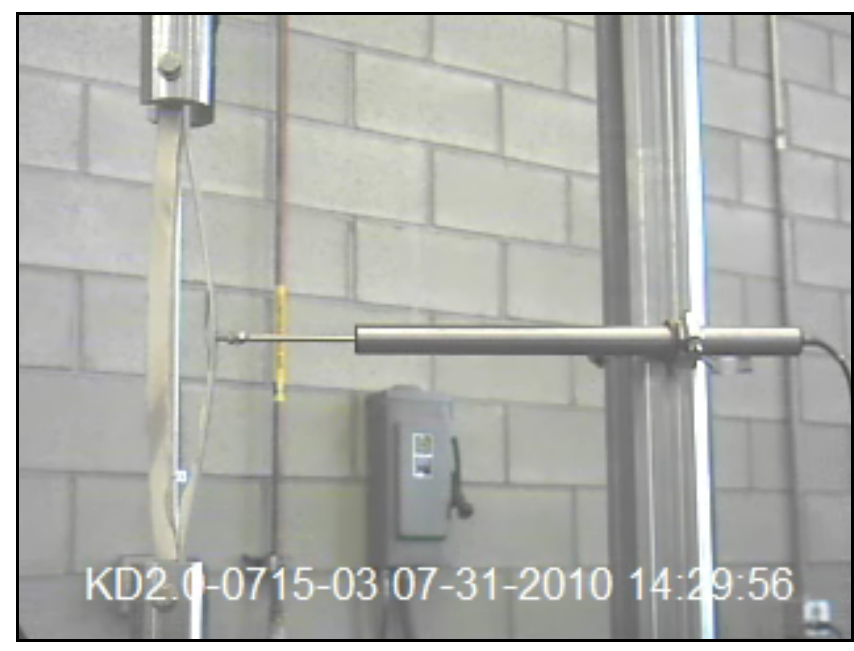

Figure 151: KD2.0 - Experimental Testing After Failure

\subsubsection{Experimental Group: Continuous Key in Horizontal Direction with Skin}

\section{Delamination Configuration}


In this particular experimental group, there are two keys used for this configuration. The keys extend the width of the composite sandwich structure and have a gap of half an inch from the edge of the delamination area to the edge of the key per each key. This is also studied per 1inch and 2-inch delamination lengths which mean the keys are shifted to provide the half an inch gap between the delaminated area and the keys. From the data in Table 31, it shows that the continuous keys placed in the horizontal direction in this configuration did not improve the structural integrity at all. In fact, it closely matches the '1.0 ID' and '2.0 ID' configurations.

Table 31: HD Configuration Comparison

\begin{tabular}{|c|c|c|c|c|}
\hline Case & $\begin{array}{c}\text { Slenderness } \\
\text { Ratio }\end{array}$ & $\begin{array}{c}\text { Maximum Vertical } \\
\text { Extension }\end{array}$ & $\begin{array}{c}\text { Maximum } \\
\text { Load }\end{array}$ & $\begin{array}{c}\text { Maximum } \\
\text { Horizontal } \\
\text { Extension }\end{array}$ \\
\hline- & - & in & lbf & in \\
\hline 11 inches & 65.39673 & 0.11083 & 685.60279 & 0.42417 \\
\hline ND & 66.58938 & 0.09881 & 737.98110 & 0.49481 \\
\hline 1.0 ID & 65.69982 & 0.03675 & 267.32638 & 0.00986 \\
\hline 2.0 ID & 65.89214 & 0.04118 & 132.60860 & 0.04519 \\
\hline HD1.0 & 64.53014 & 0.03443 & 280.36569 & 0.01353 \\
\hline HD2.0 & 65.27143 & 0.20916 & 101.60259 & 0.43025 \\
\hline
\end{tabular}

\subsection{1-Inch Delamination}

From what is seen in Figure 152, the graph does show that the test specimens remain elastic in nature until the drop at the end of the trend curves. With the inclination the vertical extension has to the load, this is a usual indicator that there is a large horizontal displacement. 


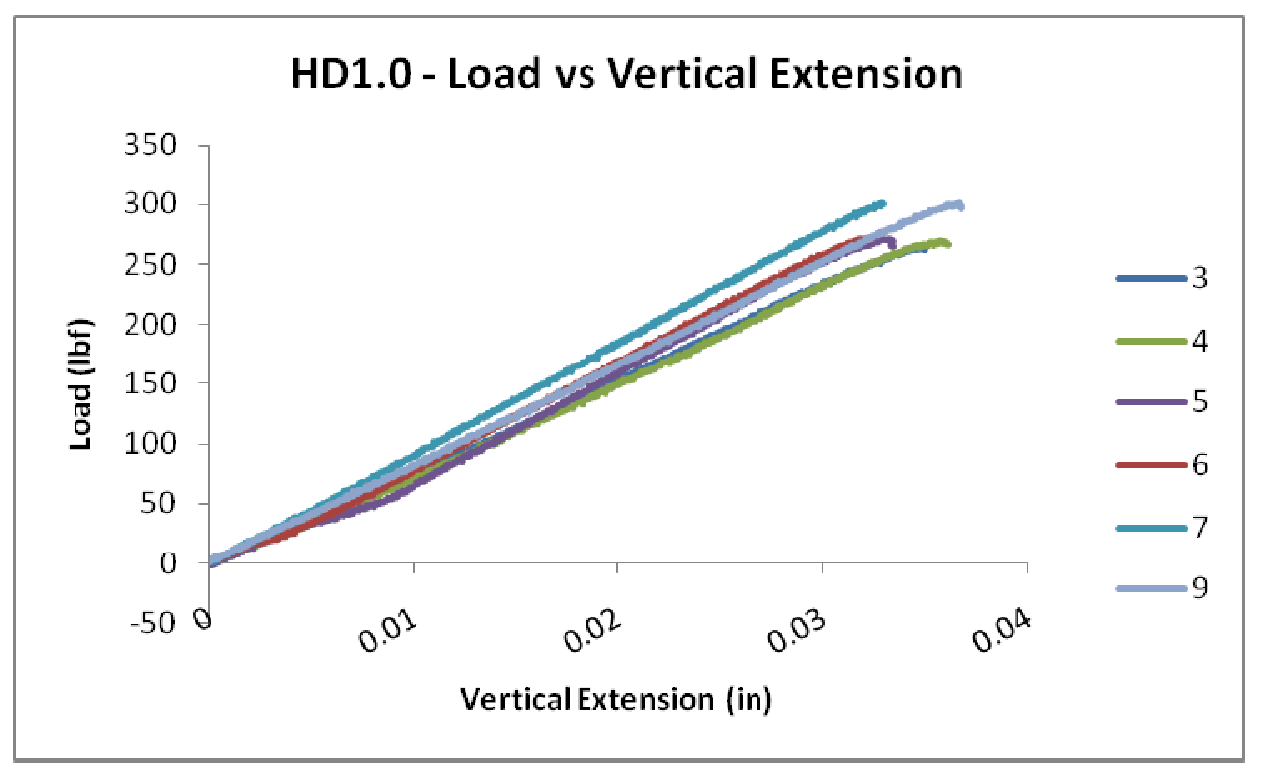

Figure 152: HD1.0 - Load Vs Vertical Extension

Figure 153 and Figure 154 differ in how the trends look due to which sides the extensometer is on. Figure 153 shows that buckling on the face sheet and the rest of structure occurred at the same time in two opposite directions. The difference between the two opposite extensions was what created the hook in the curves and indicates that one side had more extension per unit load initially and then the other side took over. As for Figure 154, it shows that the face sheet was buckling more than the rest of structure under the loading then it dramatically climbed in horizontal extension as the delamination region gave way.

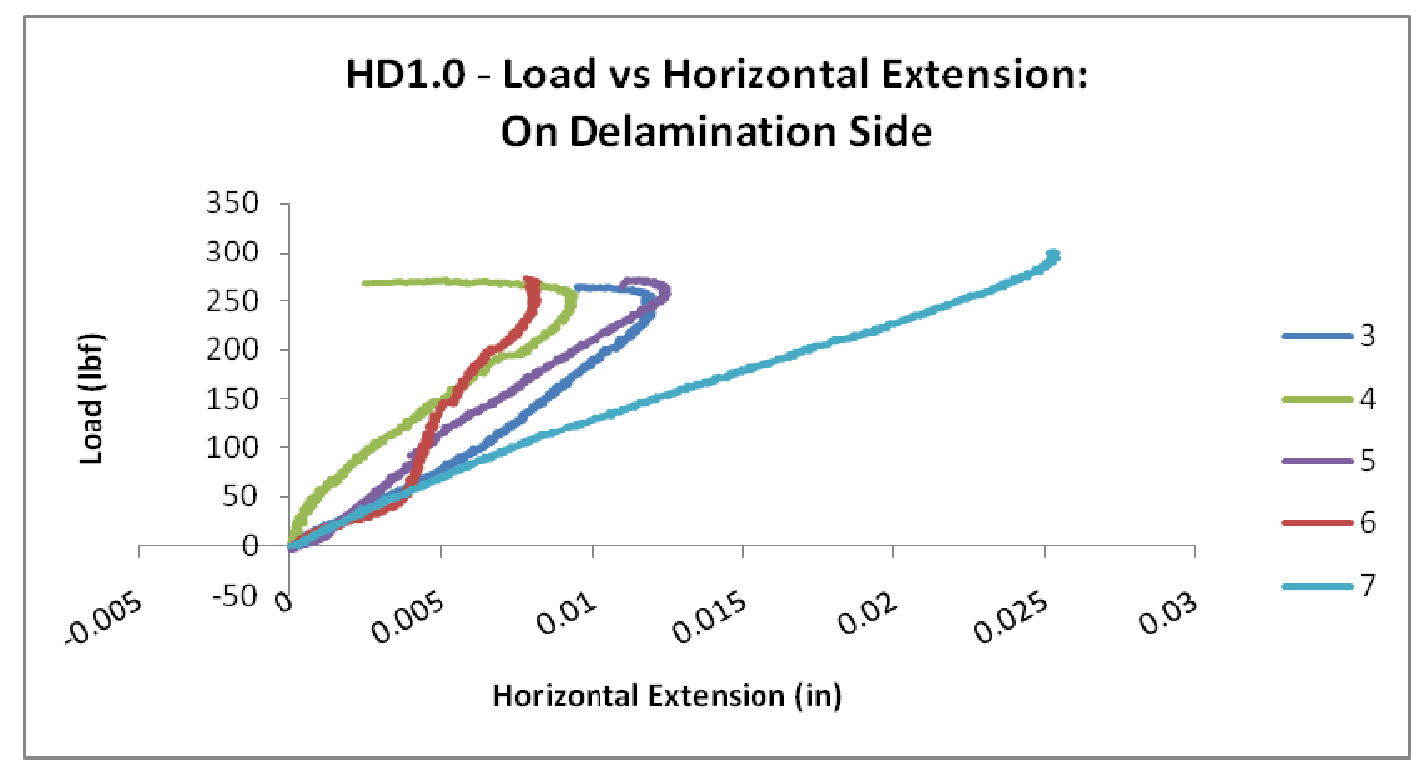


Figure 153: HD1.0 - Load Vs Horizontal Extension: On Delamination Side

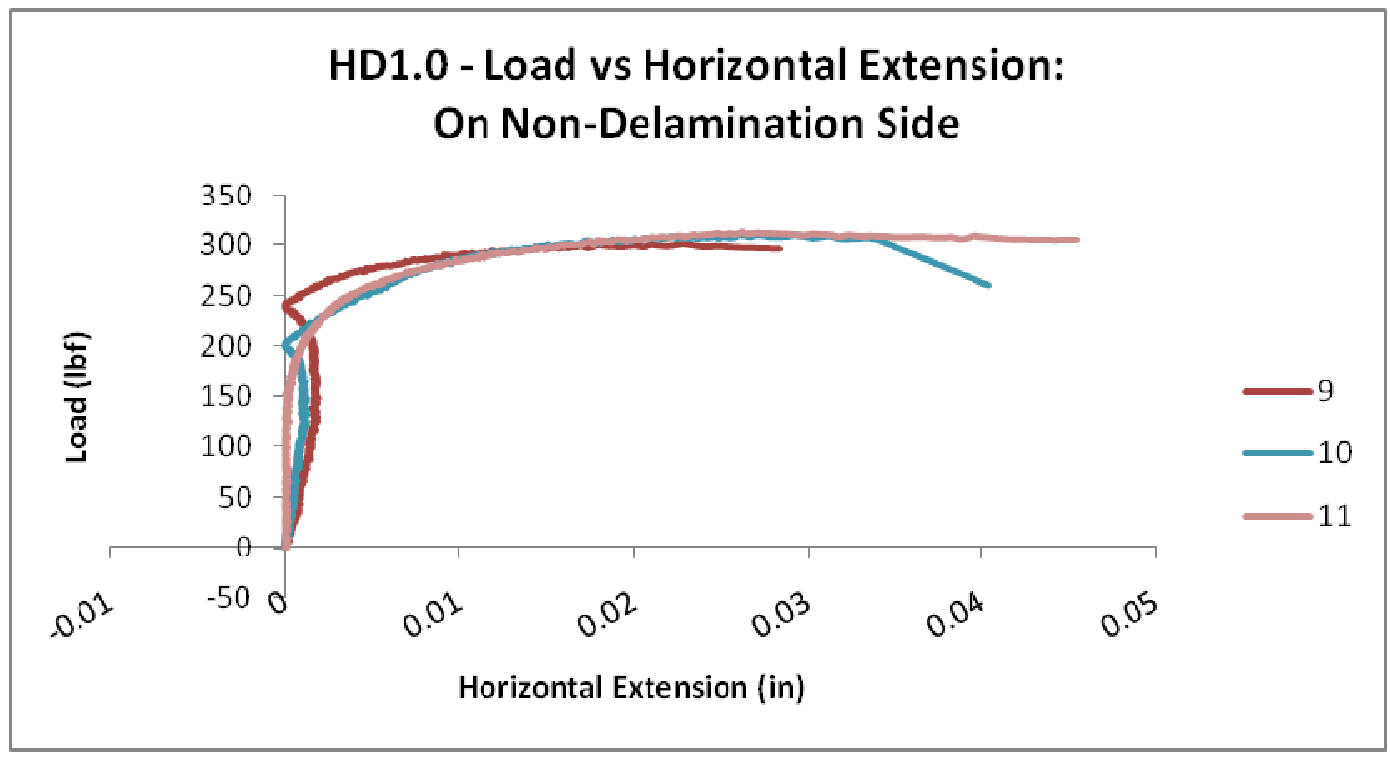

\section{Figure 154: HD1.0 - Load Vs Horizontal Extension: On Non-Delamination Side}

It is quite interesting to see such a difference between Figure 155 and Figure 156 because the two graphs show that the face sheet buckling took the lead over the non-delamination side of the structure. In Figure 155, the horizontal extension progressively got higher in value and continued to do so until the non-delamination side started to experience its own horizontal displacement. This is why the increase in horizontal displacement is seen and then followed by a drop near the end. As for Figure 156, the structure held on for a longer period of time until it gave way and started to buckle as well. This is seen near the end of the trend lines of the graph. 


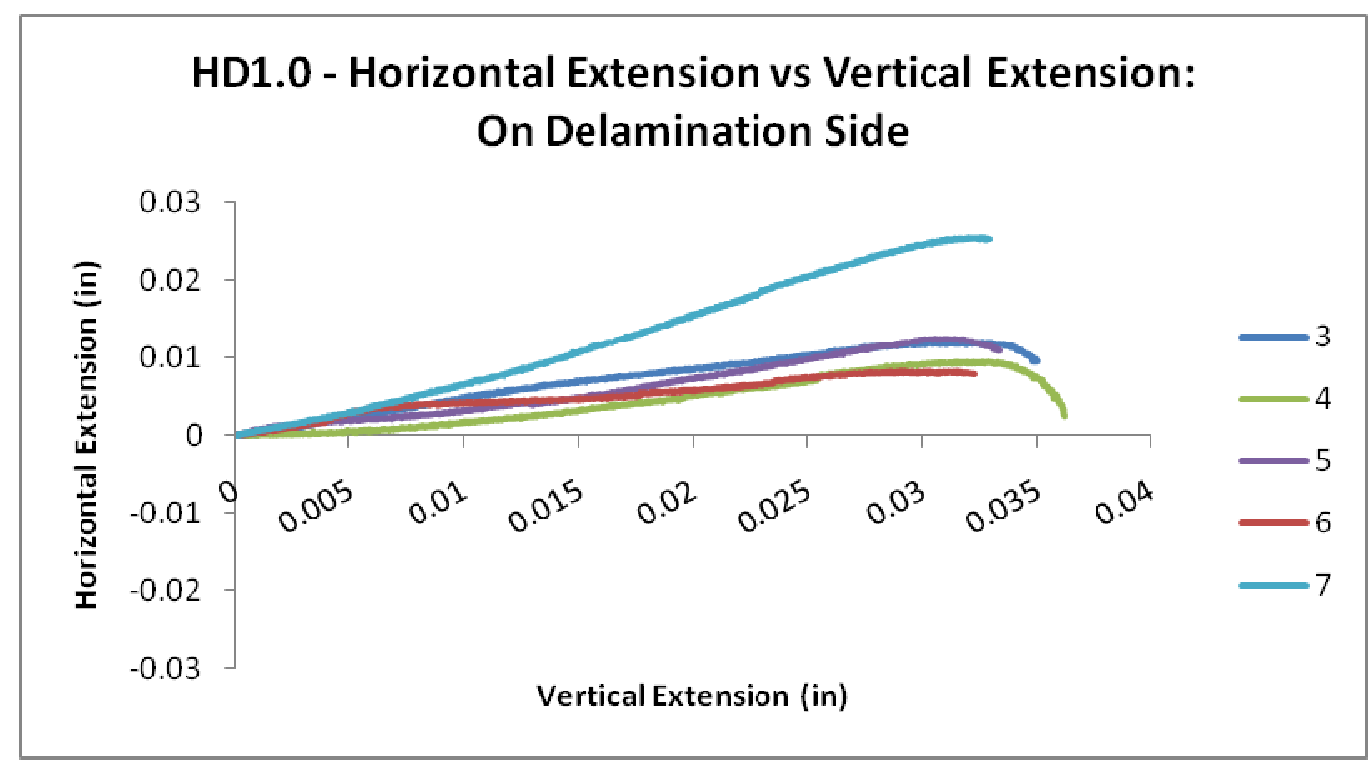

Figure 155: HD1.0 - Load Vs Horizontal Extension: On Non-Delamination Side

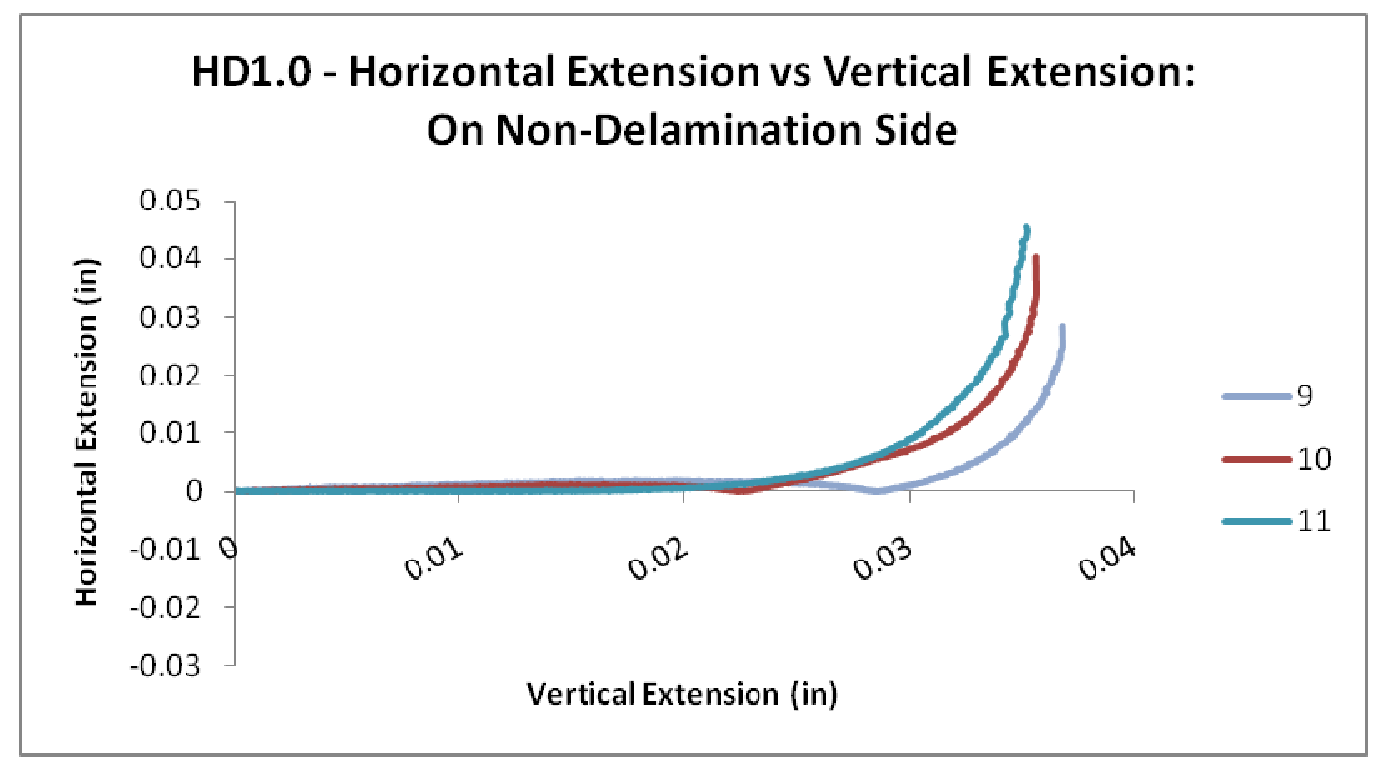

Figure 156: HD1.0 - Horizontal Extension Vs Vertical Extension: On Non-Delamination Side

Between the before and after failure figures shown in Figure 157 and Figure 158, it can seen that the test specimen would buckle when the weaker side was under compression and the non-delaminated side was in tension. Also, the after failure figure details that the horizontal key placement did not do anything to start the delaminated area from growing. This is most likely due to the half an inch gap between the delaminated region and the key. 


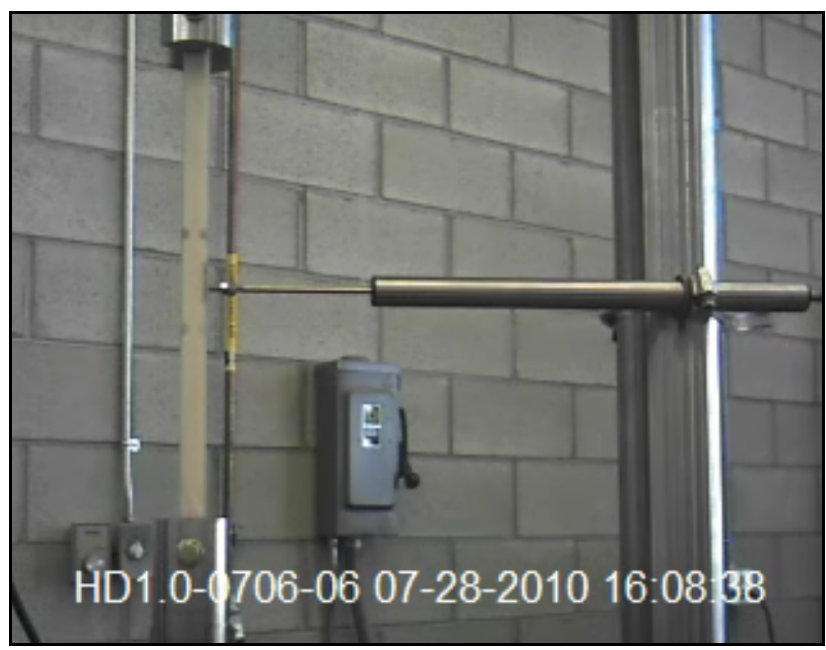

Figure 157: HD1.0 - Experimental Testing Before Failure

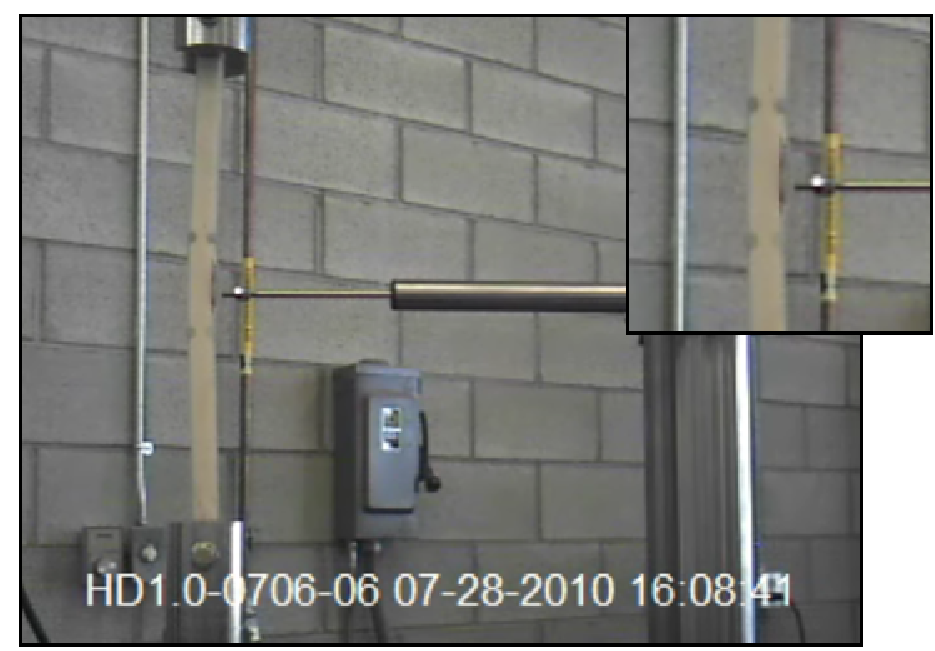

Figure 158: HD1.0 - Experimental Testing After Failure

\subsection{2-Inch Delamination}

As for the 'HD2.0' configuration, the results are a bit different from its 'HD1.0'

configuration counterpart. Figure 159 shows the load in association with the vertical extension and it displays a steep loading increase before the structures fail. This is because the delamination region started to propagate and the keys did not do anything for this configuration. This is why there is also a sharp decline right after. 


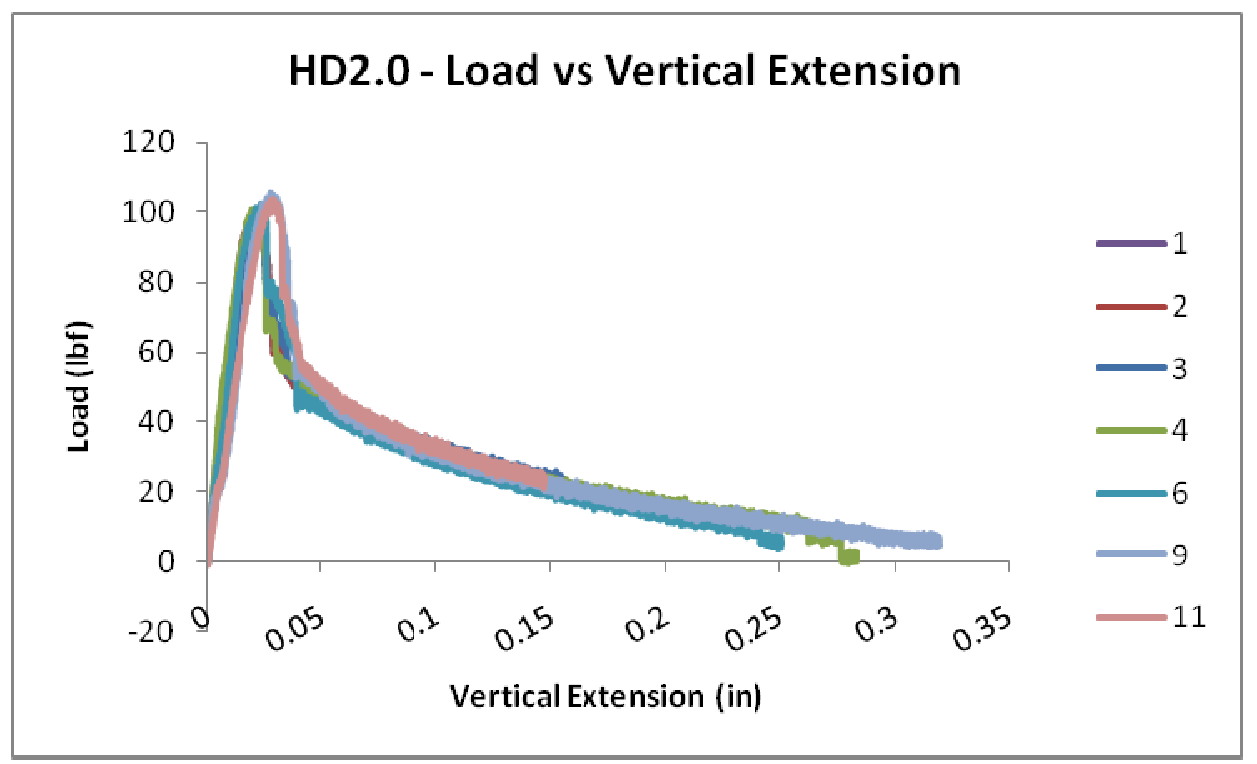

Figure 159: HD2.0 - Load Vs Vertical Extension

From placing the extensometer on the non-delamination side and the delamination side, it shows that the structure was destined to fail similar to the '2.0 ID' configuration. Figure 160 shows quite a steep increase in the loading per unit extension then dramatically declines as the delamination region propagates. As for Figure 161, the non-delamination side also shows the structure has a steep inclination in load as the displacement deforms the structure and makes it fail.

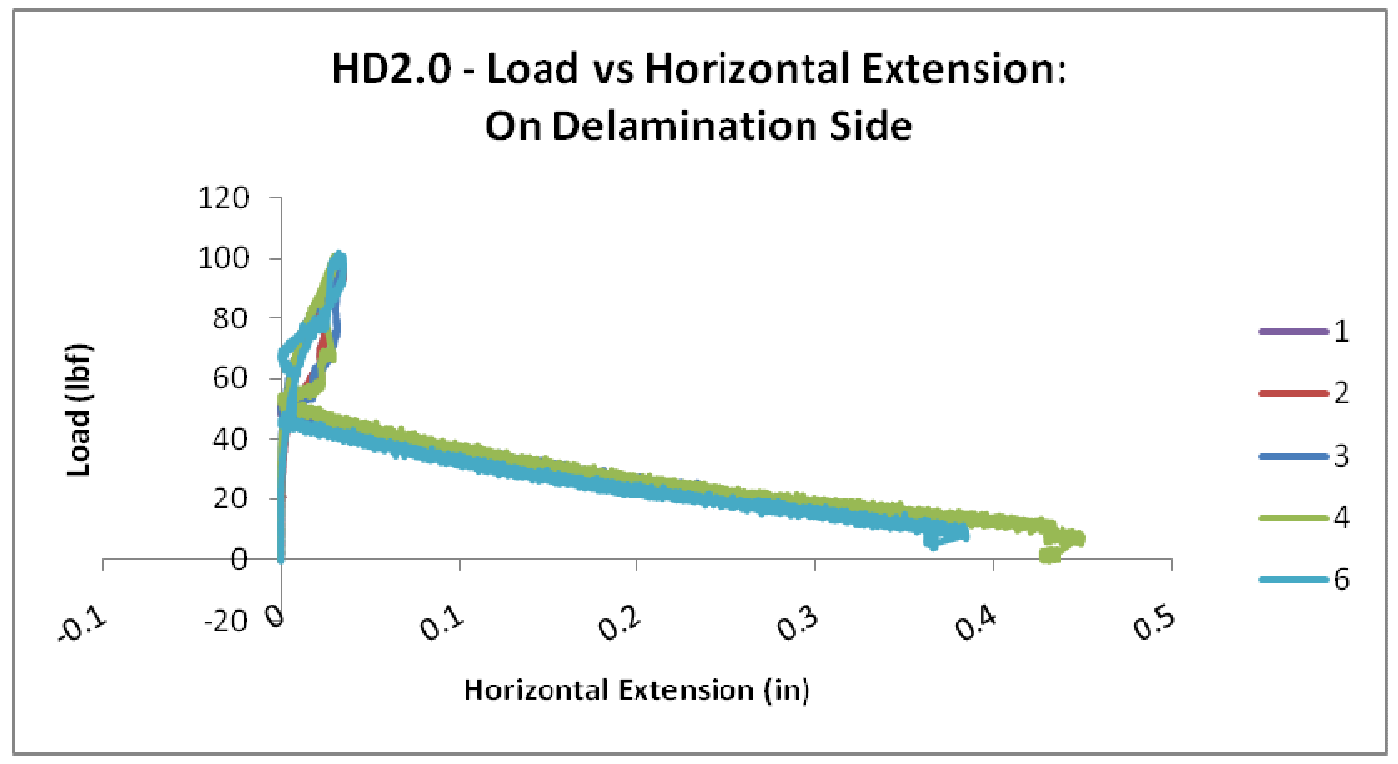

Figure 160: HD2.0 - Load Vs Horizontal Extension: On Delamination Side 


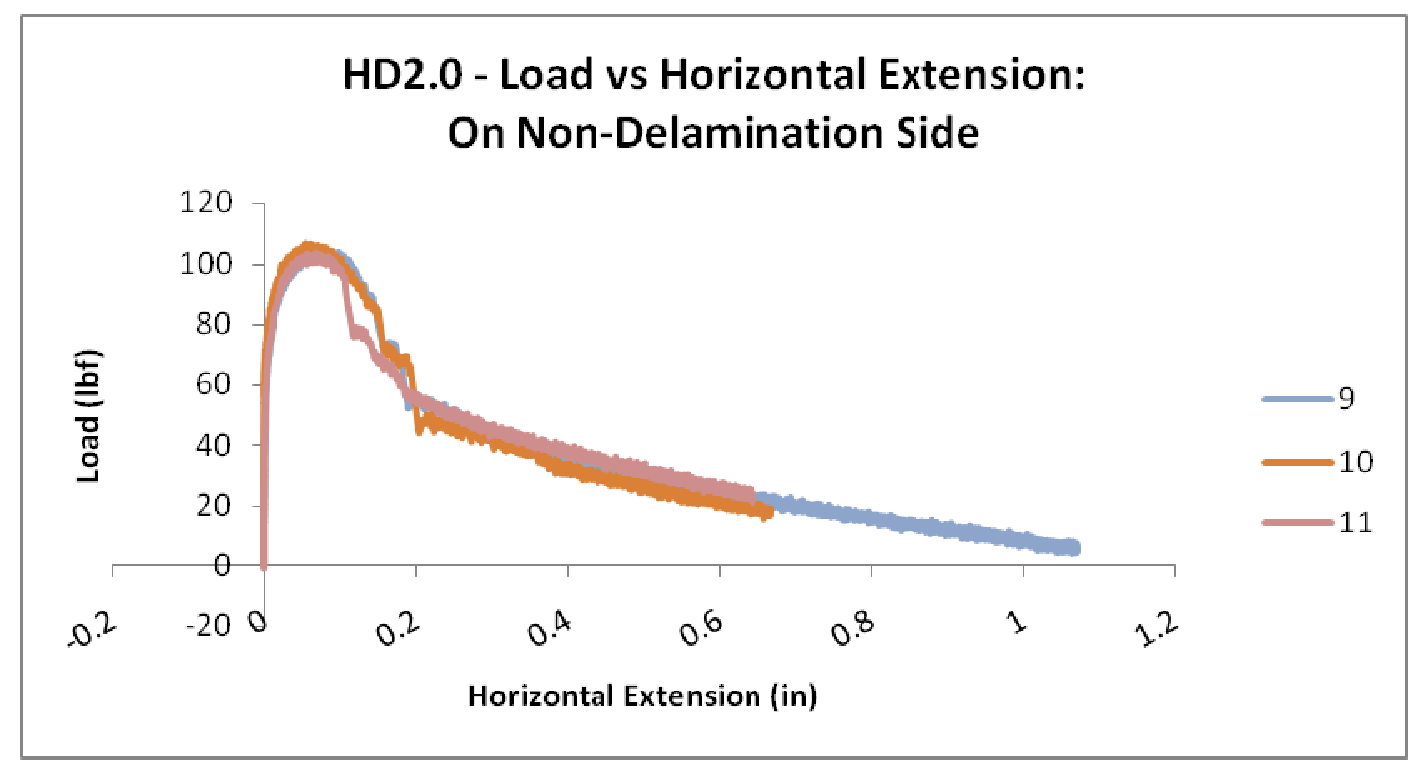

Figure 161: HD2.0 - Load Vs Horizontal Extension: On Non-Delamination Side

Figure 162 and Figure 163 details the differences between the non-delamination side and the delamination side in regards to the relationship between the horizontal extension and vertical extension. Looking at Figure 162, the curves show that there is a transition point where the buckling of the structure in one direction is subtracted from the buckling of the face sheet in the other direction before the test specimens start to see crack propagation and delamination growth. On the other hand, Figure 163 details the progressive horizontal extension of the nondelamination side as more vertical displacement is acted on the test specimens.

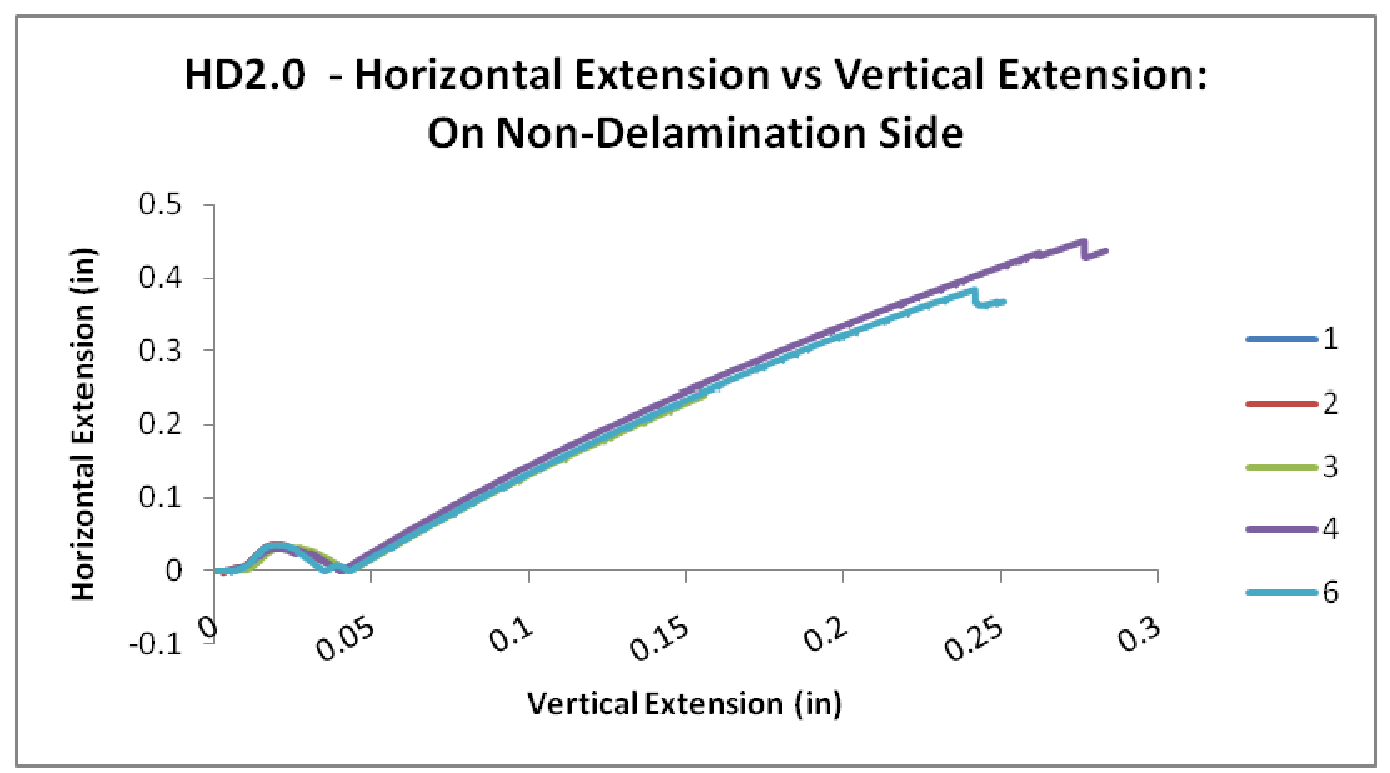


Figure 162: HD2.0 - Horizontal Extension Vs Vertical Extension: On Delamination Side

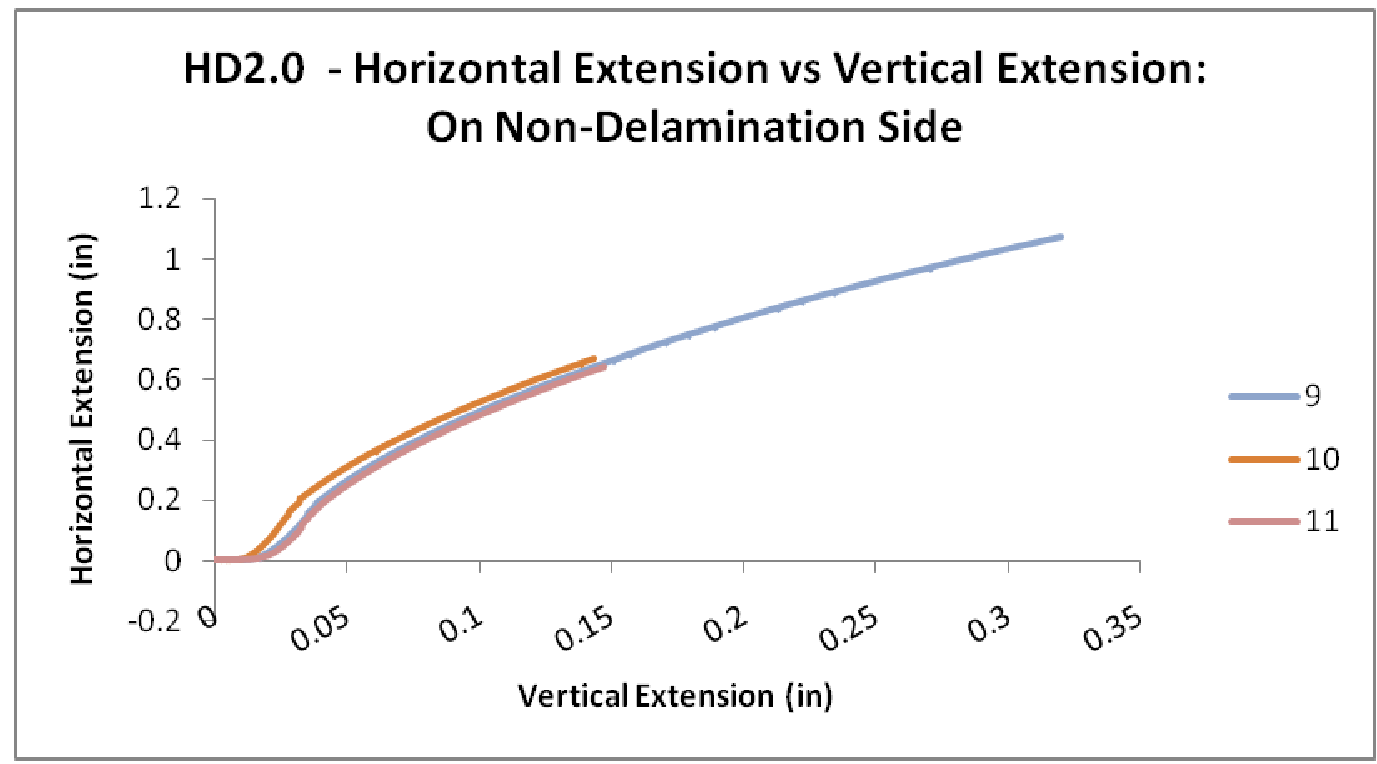

Figure 163: HD2.0 - Horizontal Extension Vs Vertical Extension: On Non-Delamination Side

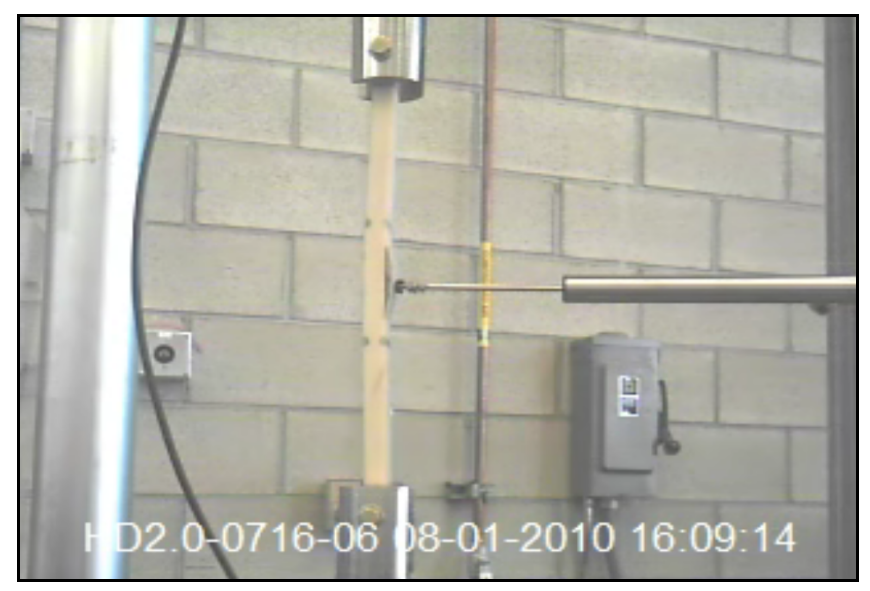

Figure 164: HD2.0 - Experimental Testing Before Failure 


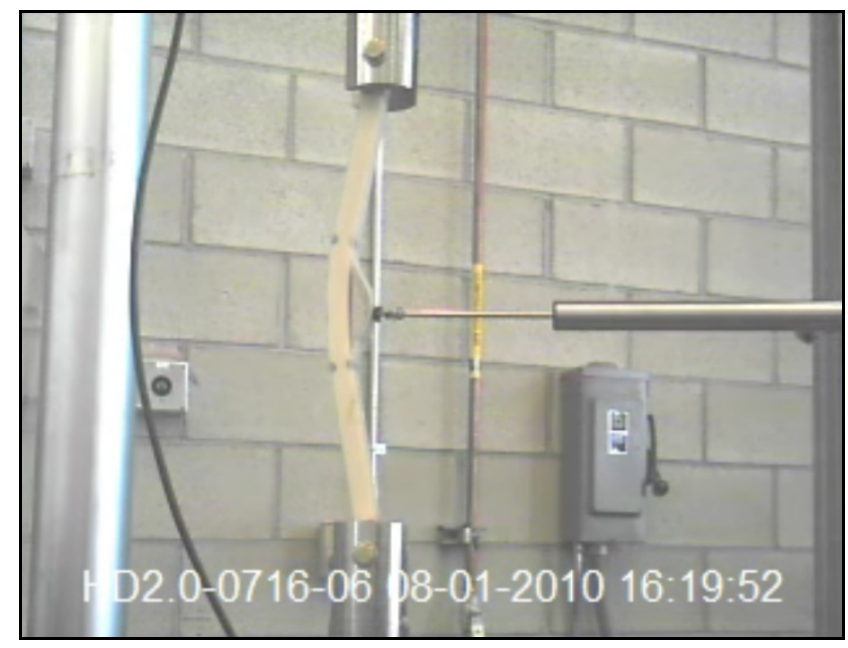

\section{Figure 165: HD2.0 - Experimental Testing After Failure}

Similar to what was seen for the 'HD1.0' configuration, the structure starts off with a slight horizontal displacement and progresses until the whole structure buckles in one direction and the skin in the other direction. This can be seen in Figure 164 and Figure 165. The placement of the keys did not deter the delaminated region from growing and this is due to placement location. 


\subsubsection{Experimental Group: Discontinuous Key in Vertical Direction with Skin}

\section{Delamination}

The last configuration with the key in the vertical direction is configured where the discontinuity starts and ends at the edges of the delamination region. The idea that inspired this configuration was to see if the added structure would provide short load transfer while reducing some weight and withstand the point load concentration within the composite sandwich structure. This was not the case. Instead the rounded end of the arrestment key on the edge of the delamination key supplied a high stress concentration and causes the test specimen to fail at that location.

In

Table 32, the data also indicates that the stress concentration resulted at lower stresses and the strains are comparable to the control group or greater. 'PL1.0' configuration is denoted by the discontinuous key in the vertical direction with 1-inch delamination length. The 'PL2.0' configuration is denoted by the discontinuous key in the vertical direction with 2-inch delamination length.

Table 32: Discontinuous Key Configuration Comparison

\begin{tabular}{|c|c|c|c|c|}
\hline Case & $\begin{array}{c}\text { Slenderness } \\
\text { Ratio }\end{array}$ & $\begin{array}{c}\text { Maximum Vertical } \\
\text { Extension }\end{array}$ & $\begin{array}{c}\text { Maximum } \\
\text { Load }\end{array}$ & $\begin{array}{c}\text { Maximum } \\
\text { Horizontal } \\
\text { Extension }\end{array}$ \\
\hline- & - & in & lbf & in \\
\hline 11 inches & 65.39673 & 0.11083 & 685.60279 & 0.42417 \\
\hline ND & 66.58938 & 0.09881 & 737.98110 & 0.49481 \\
\hline 1.0 ID & 65.69982 & 0.03675 & 267.32638 & 0.00986 \\
\hline 2.0 ID & 65.89214 & 0.04118 & 132.60860 & 0.04519 \\
\hline PL1.0 & 65.63386 & 0.03422 & 237.40629 & 0.03618 \\
\hline PL2.0 & 65.39931 & 0.08476 & 106.79098 & 0.14095 \\
\hline
\end{tabular}

\subsection{1-Inch Delamination}

The 'PL1.0' configuration lost its structural integrity because the stress point concentration gave way. From Figure 166, it is able to maintain a certain loading before the 
failure occurs right after the ultimate load is reached. The stress concentration with the combination of crack propagation produces a stepping failure as seen in the figure below. The stepping failure occurs provided that the load on the initial crack location is transferred through the arrestment key as well as the rest of structure and slowly propagates in segments as more inplane displacement is applied.

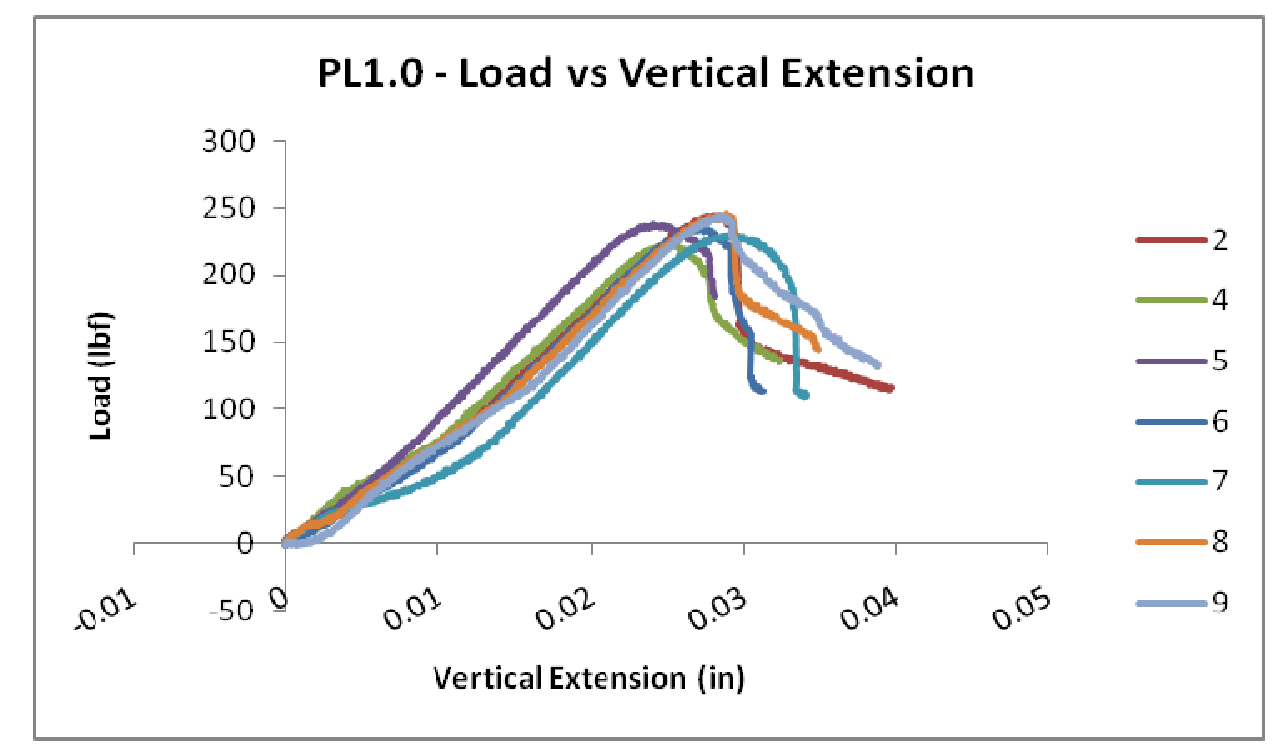

Figure 166: PL1.0 - Load Vs Vertical Extension

Figure 167 and Figure 168 are measured curves of each side of the configuration. The graph shown in Figure 167 is quite an interesting one because as the load increases, the horizontal does as well except that it sees a slight drop in loading and buckling of the face sheet. Similar to what was discussed in previous groups, the structure tends to buckle and then the face sheet follows after. In Figure 168, it is straightforward that the structure is exhibiting buckling. 


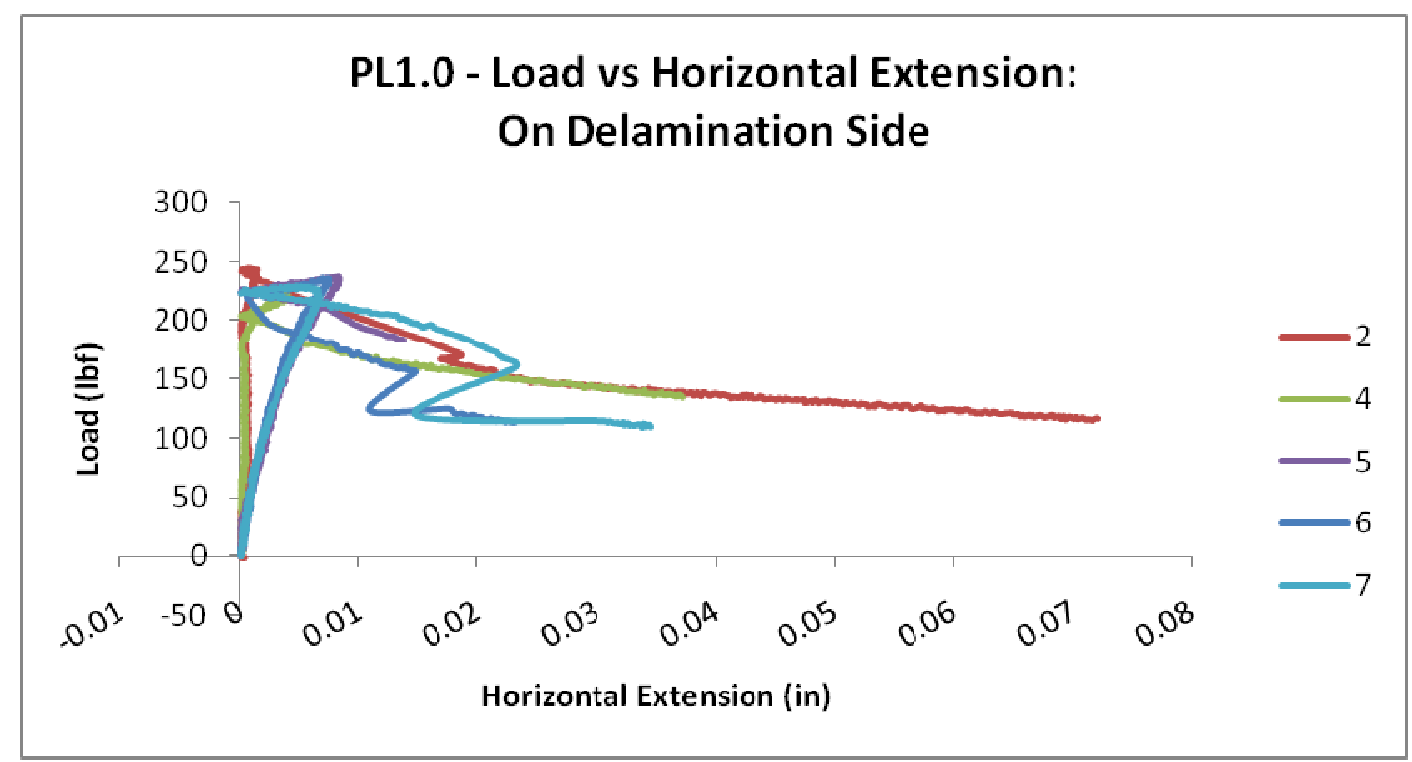

Figure 167: PL1.0 - Load Vs Horizontal Extension: On Delamination Side

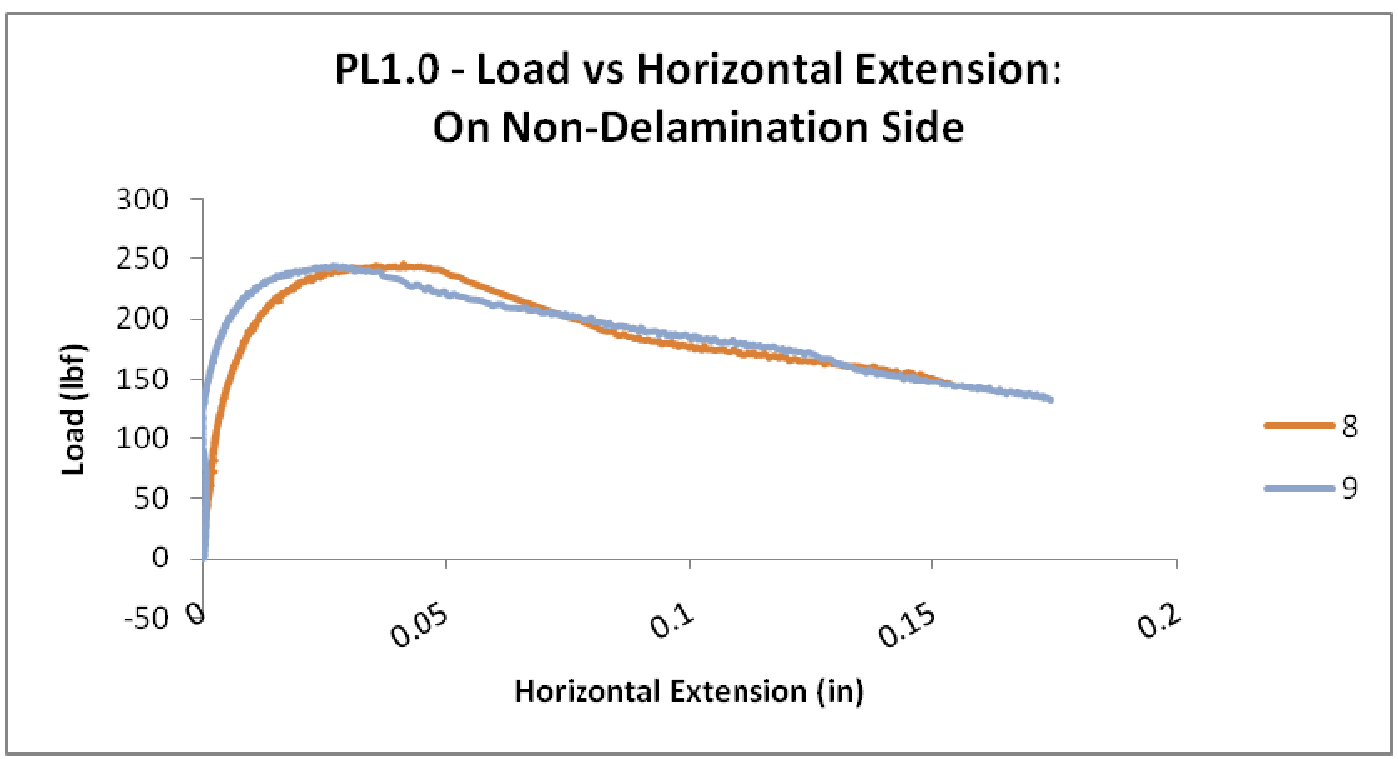

Figure 168: PL1.0 - Load Vs Horizontal Extension: On Non-Delamination Side

From Figure 169 and Figure 170, it can be seen that the horizontal extension compared with the vertical extension differs quite a bit between the non-delamination side and the delamination side. Figure 169, which is the delamination side, indicates that there is buckling on the face sheet but it has a shorter displacement in comparison to its control counterparts. Figure 170 , which is the non-delamination side, also shows buckling in effect with the larger geometry. The point load concentration on the delamination side of the composite sandwich structure 
starts to fail while the other side would be able to continue buckling if there was no failure on the delamination side. The shape of the curvature in Figure 169 entails a quicker failure while the non-delamination side has more of a round trend line.

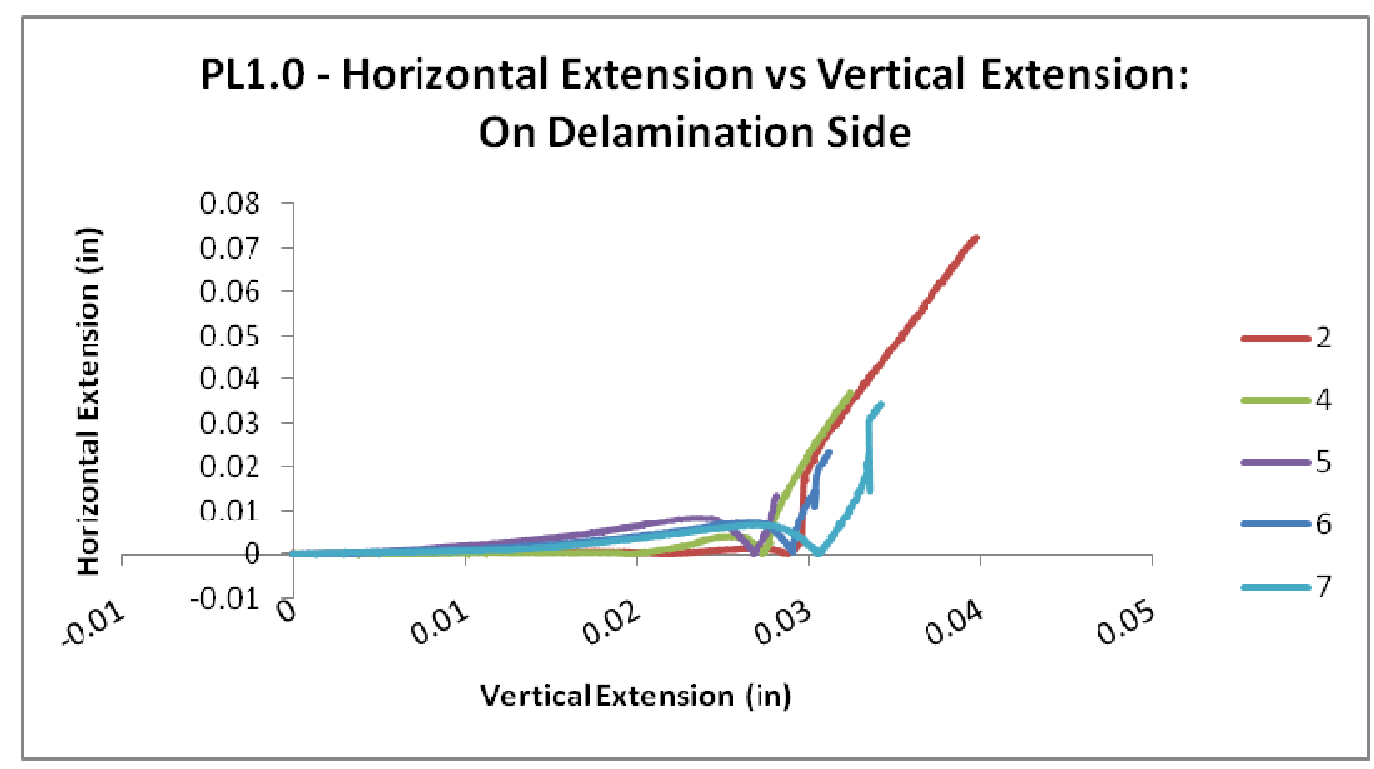

Figure 169: PL1.0 - Horizontal Extension Vs Vertical Extension: On Delamination Side

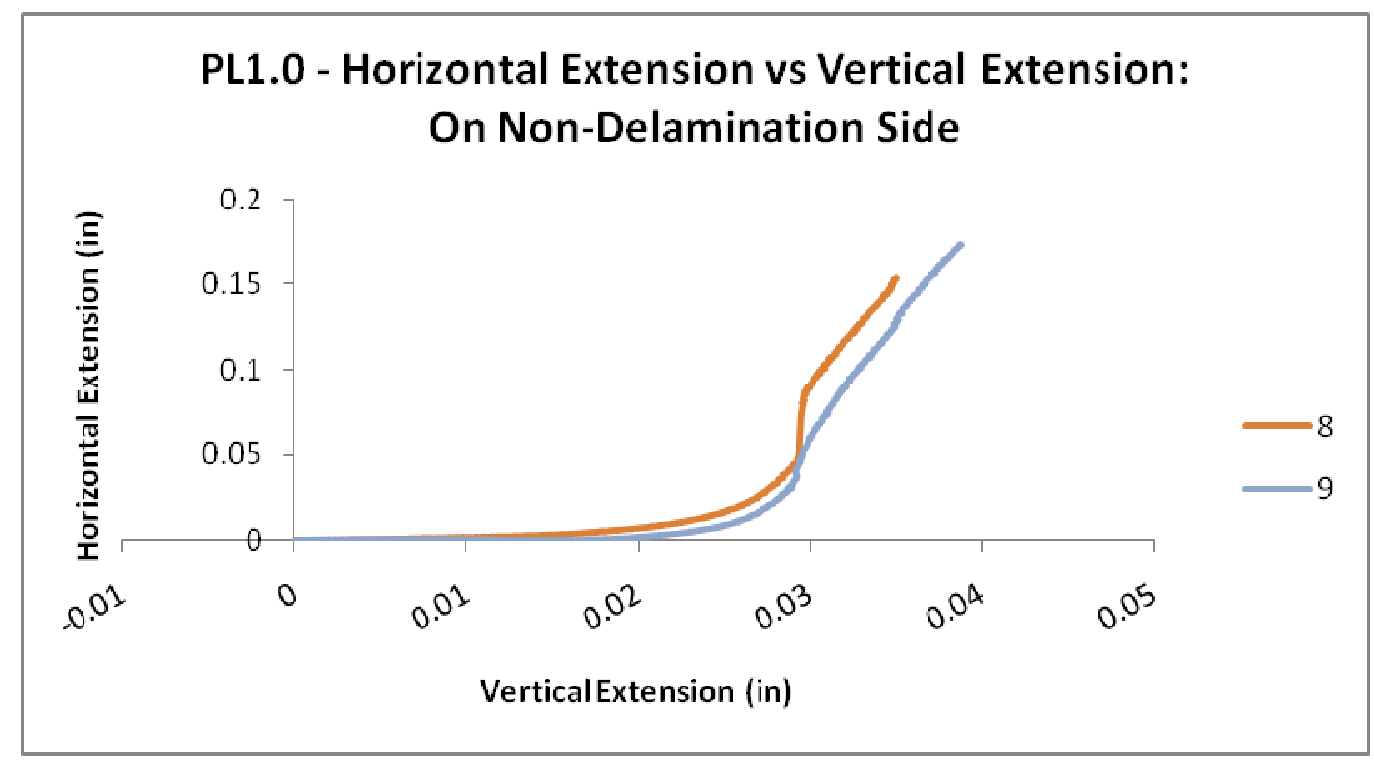

Figure 170: PL1.0 - Horizontal Extension Vs Vertical Extension: On Non-Delamination Side

It can be seen from Figure 171 that the test specimen shows buckling in both directions. That is, the face sheet is buckling one direction while the rest of the structure is buckling in the 
opposite direction. The horizontal displacement is slightly shorter than the initial delamination control group specimens.

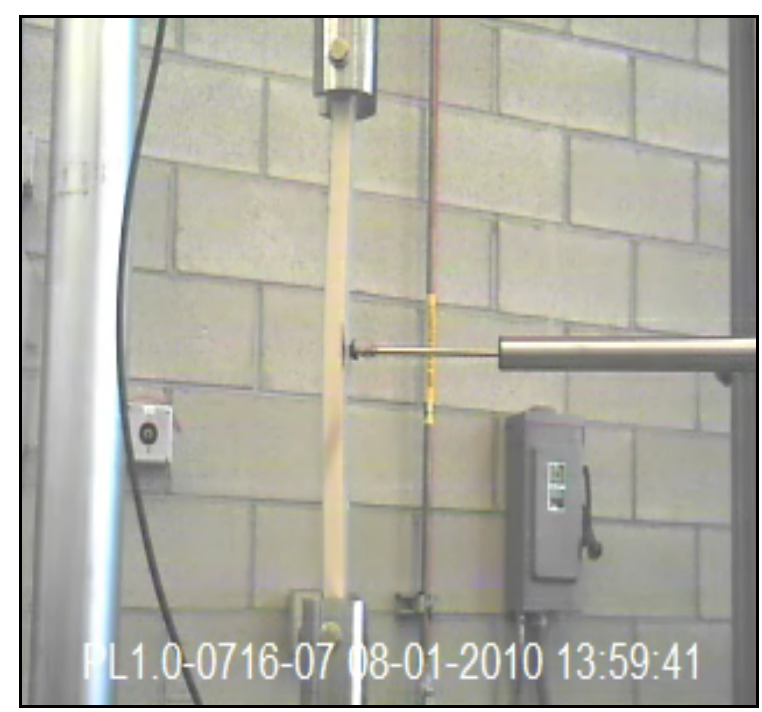

\section{Figure 171: PL1.0 - Experimental Testing Before Failure}

The reason for the shorter horizontal extension is because the test specimens are failing earlier due to the high stress concentration at the end of the key near the delamination region. This is viewable in the right image in Figure 172. The image shows a debond happening as well when the test specimen is loaded.

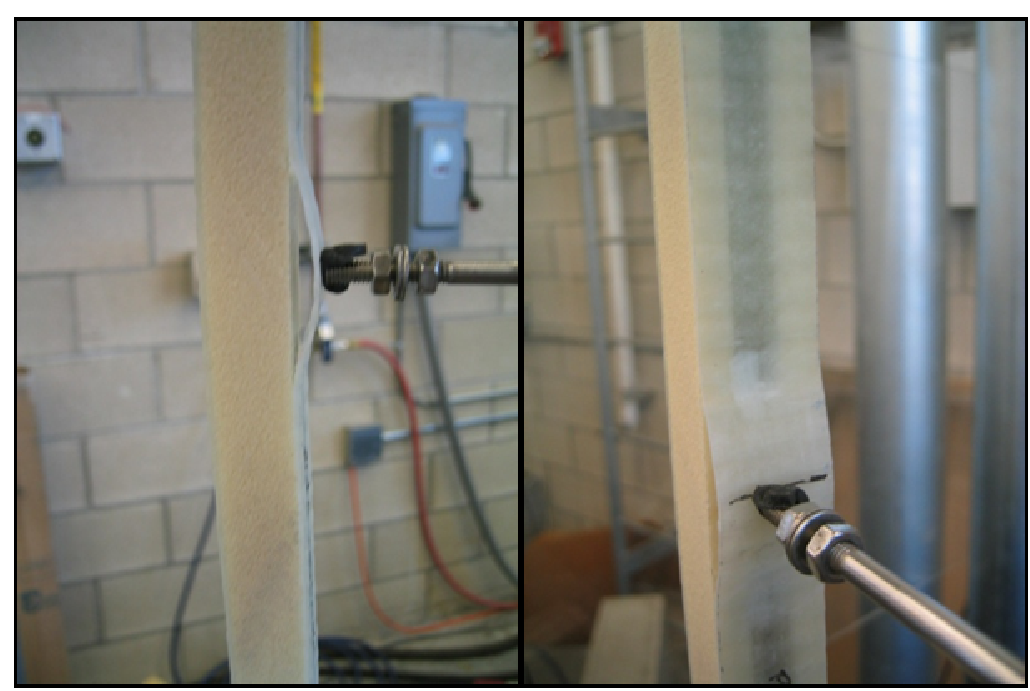

Figure 172: PL1.0 - Experimental Testing After Failure 


\subsection{2-Inch Delamination}

The 'PL2.0' configuration refers to the discontinuous key configuration with 2.0-inch delamination length. From Figure 173, it can be seen that the graph shows that structure exhibits more buckling because of the large delaminated area, which is followed by longer horizontal displacement.

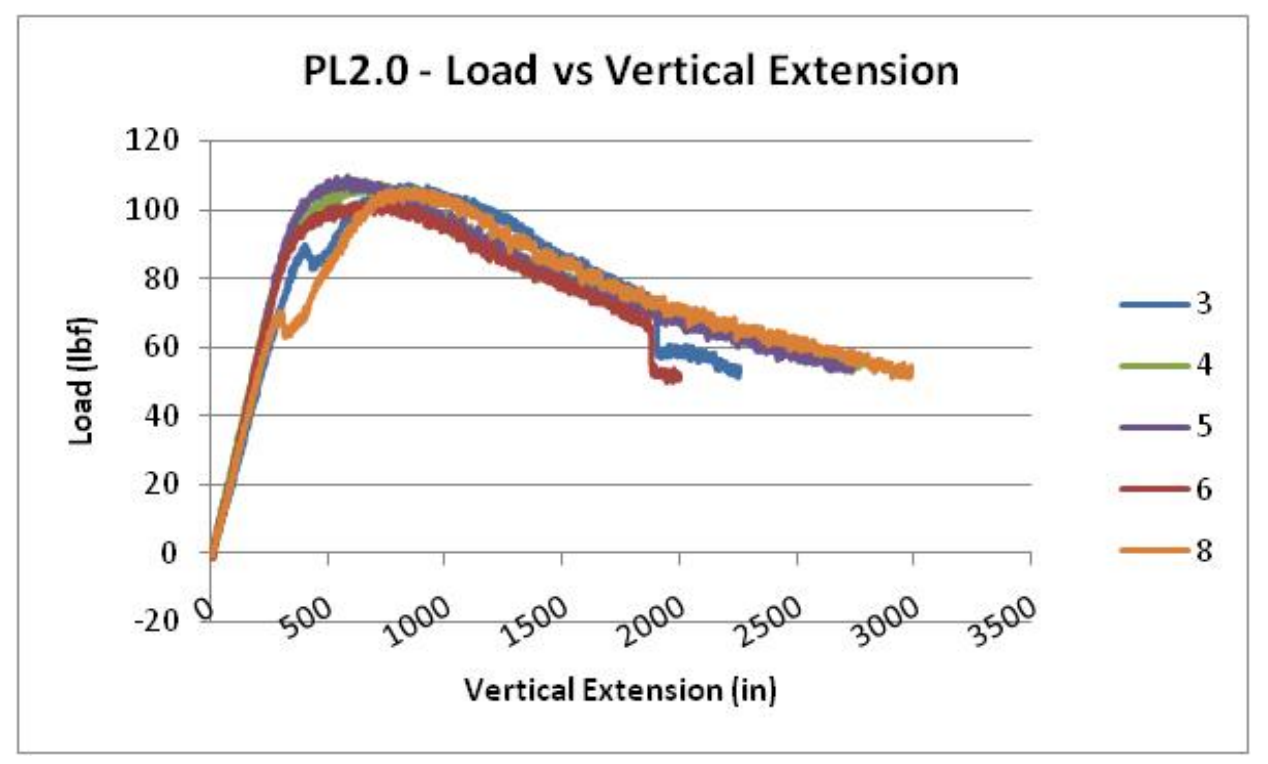

Figure 173: PL2.0 - Load Vs Vertical Extension

The horizontal extension associated with the loading shows that the structure is exhibiting buckling of the face sheet immediately after the structure is loaded. This is because the buckled skin is carrying more loads and unable to transfer the load elsewhere thus the failure occurs at the point location. Figure 174 details this behavior. As for Figure 175, this figure shows that buckling also occurs immediately after the structure has been loaded. 


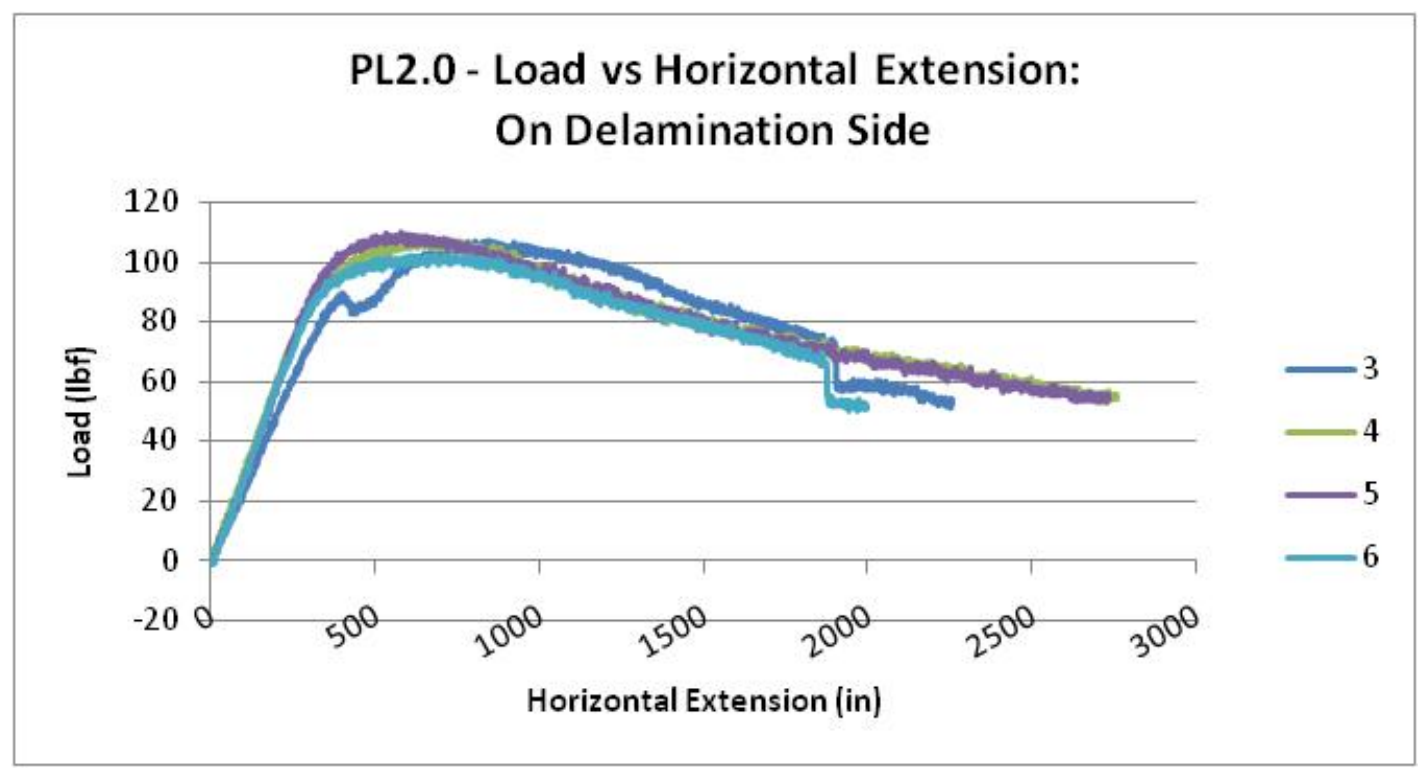

Figure 174: PL2.0 - Load Vs Horizontal Extension: On Delamination Side

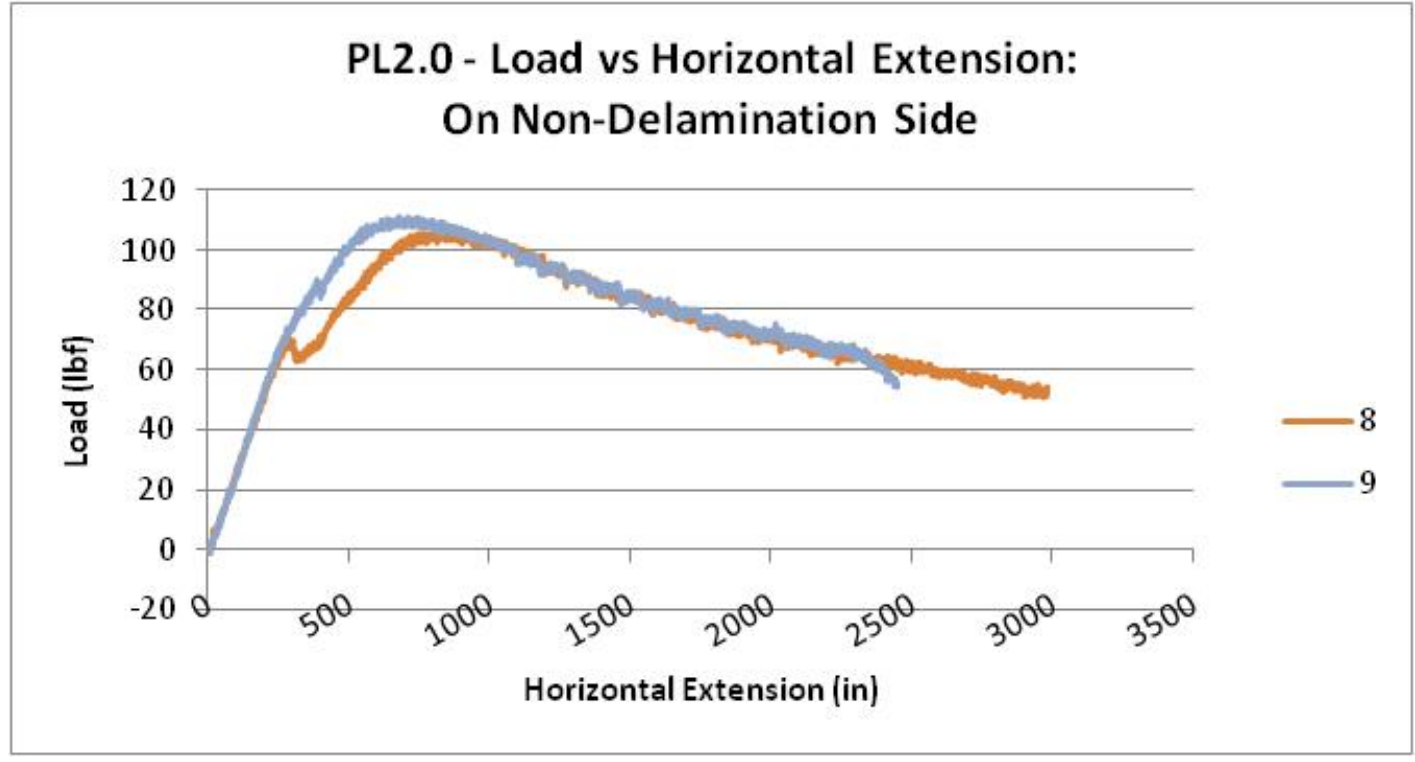

Figure 175: PL2.0 - Load Vs Horizontal Extension: On Non-Delamination Side

Figure 176 details the relationship between the horizontal extension and its vertical extension counterpart. The graph shows that the whole structure is in buckling mode when the structures are loaded and the separation between the skin and core happens immediately under loading. The buckling structure and buckling face sheet are bowing in two opposite directions where the rest of the structure bows faster than the face sheet. On the other hand, Figure 177 details how the rest of structure buckles and displaces in the horizontal direction only. 


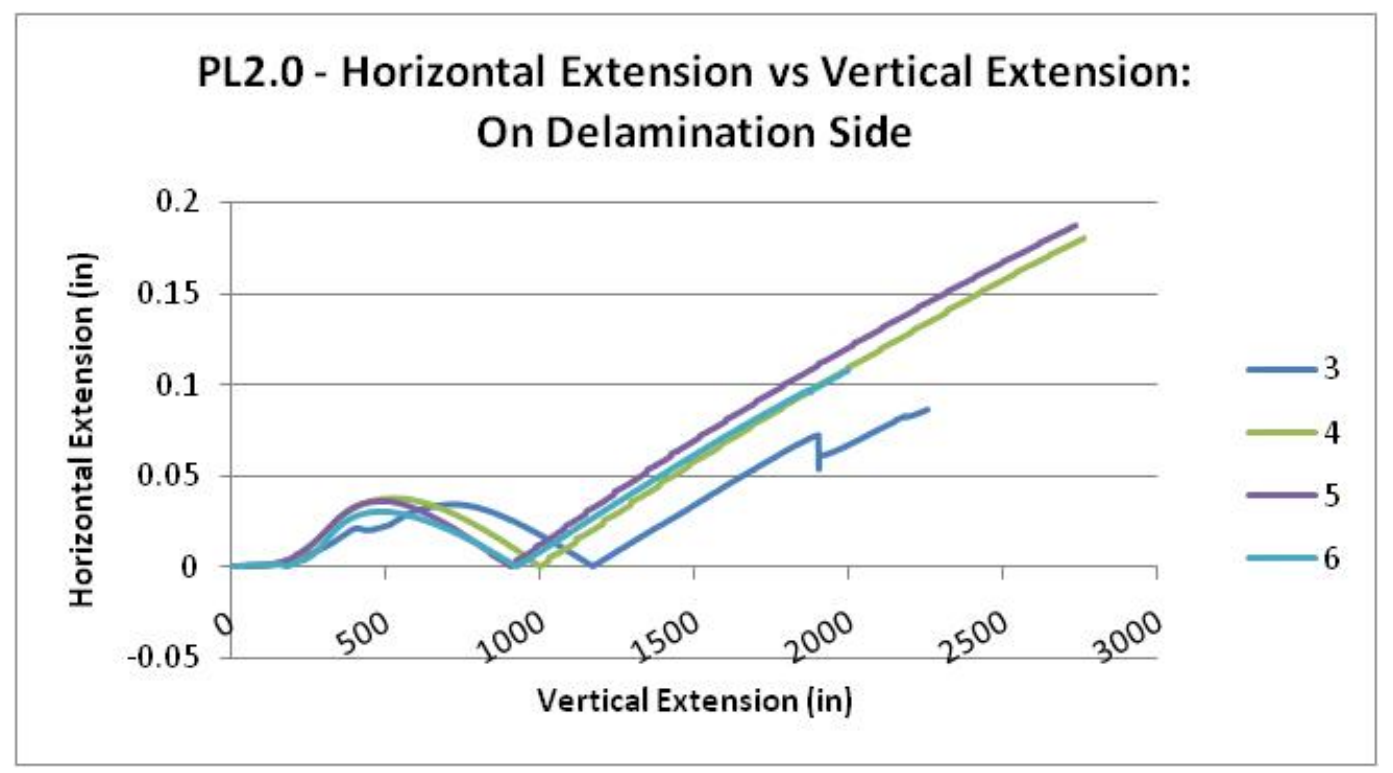

Figure 176: PL2.0 - Horizontal Extension Vs Vertical Extension: On Delamination Side

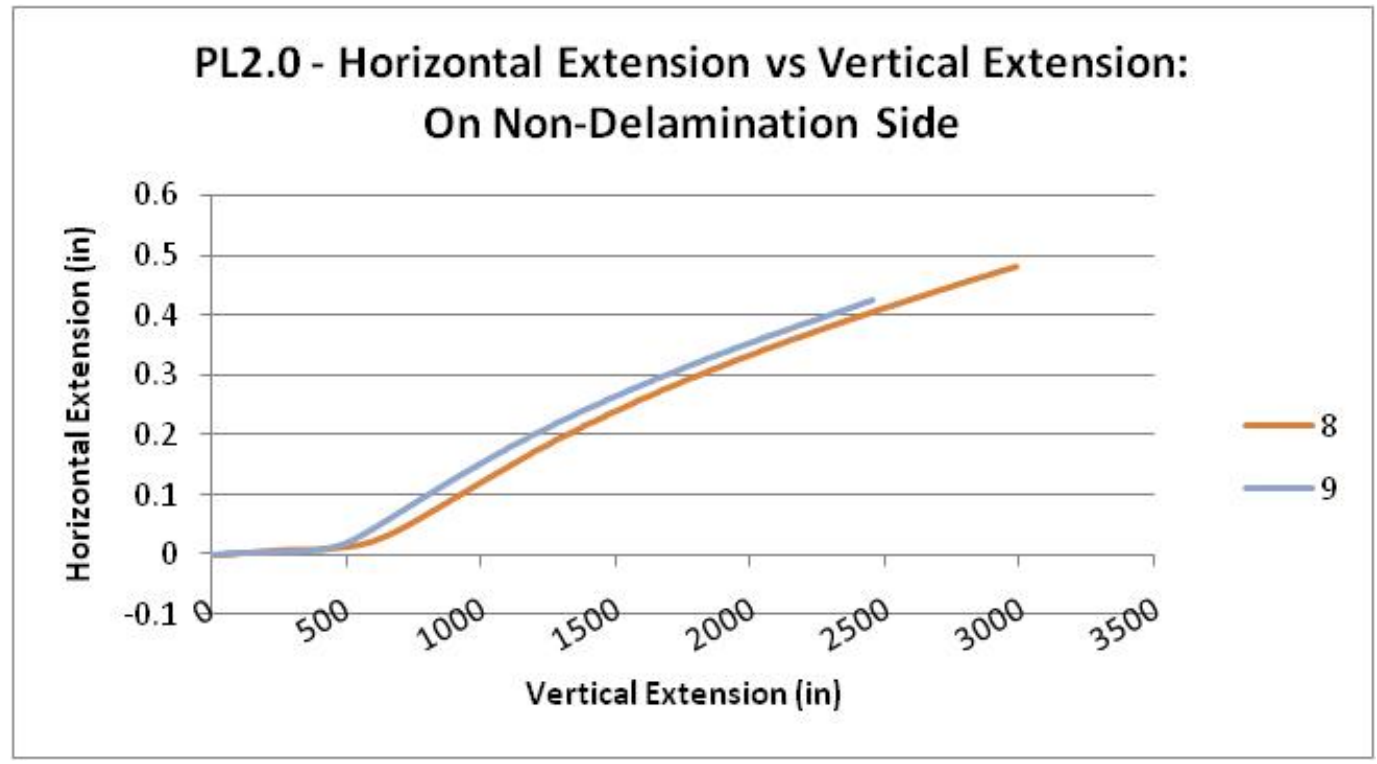

Figure 177: PL2.0 - Horizontal Extension Vs Vertical Extension: On Non-Delamination Side 


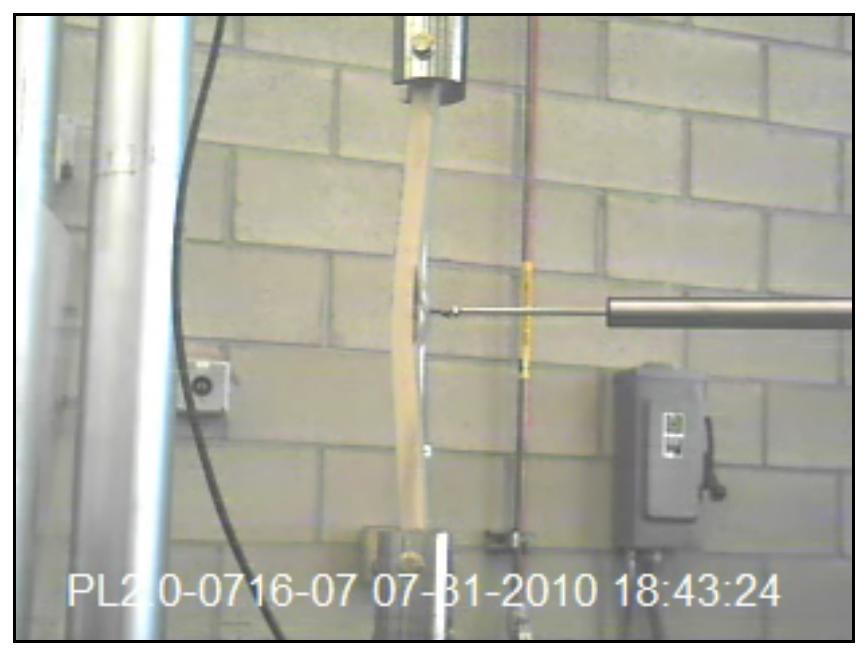

Figure 178: PL2.0 - Experimental Testing Before Failure

The 'PL2.0' configuration shown in Figure 178 presented a slight horizontal displacement as the test specimen became loaded and vertically displaced. This caused the buckling of the face sheet and the rest of the structure to happen in two opposite directions. As the vertical displacement continues, Figure 179 shows failures happening at the edge of the delaminated area and the ends of the arrestment key. This is because the key became a stress point concentration and explains the limited loading capacity.

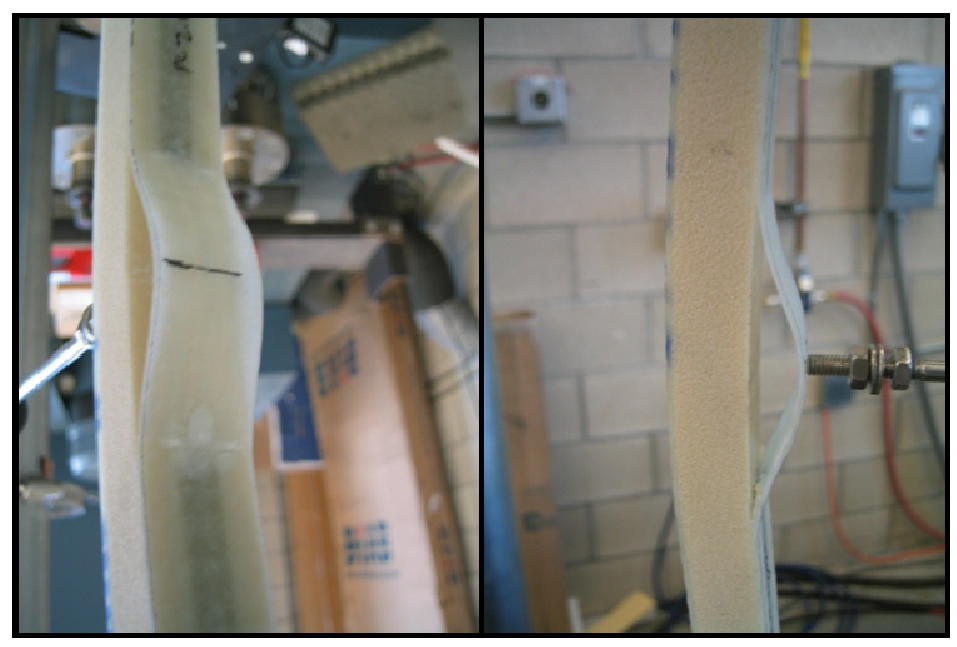

Figure 179: PL2.0 - Experimental Testing After Failure

\subsection{Comparison of Configurations}

The point of the comparison of the configuration section of this report is to show the similarities and differences of these groups to one another. These groups are divided into 
subsections and will be compared based on features that were investigated. Several variables that will be looked into include load capacity, the vertical and horizontal extensions, and their failure modes. Graphs for the comparison of all of these configurations can be found in the Appendix. The groups are divided as follow:

- Comparison between '11 inches' and 'ID' Configurations

- Comparison between ' 11 inches' and 'ND' Configurations

- Comparison between 'ND', 'SD', and 'KD' Configurations

- Comparison between 'ID', 'PL', and 'HD' Configurations

Comparison between '11 inches' and 'ID' Configurations - The comparison between these two configurations are mainly to see how an initial delamination introduced into the structure will affects its behaviors.

Comparison between ' 11 inches' and 'ND' Configurations - The comparison between these two configurations is mainly to see how the embedment of the delamination arrestment key will affect the outcome of the composite sandwich structure.

Comparison between ' $N D$ ', 'SD', and ' $K D$ ' Configurations - The comparison between these three configurations is mainly to see if the embedment of the delamination arrestment key has any effects on the behavior if there is an initial delamination where the skin is delaminated from the key and foam core. Also, an examination of the test specimen with an initial delamination is done to see its behavior where the arrestment key and skin is delaminated from the foam core.

Comparison between ' $I D$ ', 'PL', and 'HD' Configurations - The comparison between these three configurations is mainly to see the behaviors of the structures when adding a discontinuous pattern for the arrestment key to an initially delaminated composite sandwich structure. Also, this section will compare what happens when the arrestment keys are placed horizontally. 


\subsubsection{Comparison between ' 11 inches' and 'ID' Configurations}

Table 33: Comparison between ' 11 inches' and 'ID' Configurations

\begin{tabular}{|c|c|c|c|c|}
\hline Case & $\begin{array}{c}\text { Slenderness } \\
\text { Ratio }\end{array}$ & $\begin{array}{c}\text { Maximum } \\
\text { Vertical } \\
\text { Extension }\end{array}$ & $\begin{array}{c}\text { Maximum } \\
\text { Load }\end{array}$ & $\begin{array}{c}\text { Maximum Horizontal } \\
\text { Extension }\end{array}$ \\
\hline- & - & in & lbf & in \\
\hline 11 inches & 65.39673 & 0.11083 & 685.60279 & 0.42417 \\
\hline 1.0 ID & 65.69982 & 0.03675 & 267.32638 & 0.00986 \\
\hline 2.0 ID & 65.89214 & 0.04118 & 132.60860 & 0.04519 \\
\hline
\end{tabular}

With any forms of delamination within the composite sandwich structure, the structure loses all its structural integrity. This can be seen in the table above. The loading capacity drops over $50 \%$ for the delaminated configuration without any embedment. It gets even worse as the size of the delamination increases.

The 'ID' configurations also fail at shorter vertical and horizontal extensions. The shorter extensions show that the structure is not even able to transfer the loads, but creates a high stress concentration at the delaminated region. These initial delaminated test specimens without any embedment can be generalized as nonconforming and a fail structure.

\subsubsection{Comparison between ' 11 inches' and 'ND' Configurations}

Table 34: Comparison between '11 inches' and 'ND' Configurations

\begin{tabular}{|c|c|c|c|c|}
\hline Case & $\begin{array}{c}\text { Slenderness } \\
\text { Ratio }\end{array}$ & $\begin{array}{c}\text { Maximum } \\
\text { Vertical } \\
\text { Extension }\end{array}$ & $\begin{array}{c}\text { Maximum } \\
\text { Load }\end{array}$ & $\begin{array}{c}\text { Maximum Horizontal } \\
\text { Extension }\end{array}$ \\
\hline- & - & in & lbf & in \\
\hline ND & 66.58938 & 0.09881 & 737.98110 & 0.49481 \\
\hline 11 inches & 65.39673 & 0.11083 & 685.60279 & 0.42417 \\
\hline
\end{tabular}

The first noticeable difference between the two different configurations is the loading capacity. The insertion of the arrestment key allowed for the composite sandwich structure to carry more loading than the non-delaminated and non-embedded test specimens. Looking at the vertical extension between the two configurations, the 'ND' configuration has a shorter displacement yet a higher loading capacity. The embedment of the higher strength material, the 
cross-sectional shape, and how the key was placed defines the increase in loading capacity and explains why the displacement is much shorter.

The horizontal extension differs though when the ' 11 inches' configuration has a shorter horizontal extension than the 'ND' configuration. This is a response to the delamination arrestment key carrying and transferring the loading much easier. Also, fiberglass composite material is known to have great flexure properties.

\subsubsection{Comparison between 'ND', 'SD', and 'KD' Configurations}

Table 35: Comparison between 'ND', 'SD', and 'KD' Configurations

\begin{tabular}{|c|c|c|c|c|}
\hline Case & $\begin{array}{c}\text { Slenderness } \\
\text { Ratio }\end{array}$ & $\begin{array}{c}\text { Maximum Vertical } \\
\text { Extension }\end{array}$ & $\begin{array}{c}\text { Maximum } \\
\text { Load }\end{array}$ & $\begin{array}{c}\text { Maximum } \\
\text { Horizontal Extension }\end{array}$ \\
\hline- & - & in & lbf & in \\
\hline ND & 66.58938 & 0.09881 & 737.98110 & 0.49481 \\
\hline SD1.0 & 65.63689 & 0.08530 & 604.48262 & 0.30740 \\
\hline SD2.0 & 64.88100 & 0.10069 & 680.74380 & 0.28825 \\
\hline KD1.0 & 65.12548 & 0.08611 & 864.98802 & 0.56654 \\
\hline KD2.0 & 66.27747 & 0.06617 & 746.09667 & 0.27680 \\
\hline
\end{tabular}

The 'SD' and 'KD' configurations show that majority of the structural integrity is maintained and could serve as a possible alternative to repairing composite sandwich structures with a foam core.

From Table 35, the 'SD' configuration shows a load drop but not as severe as if it was from a delaminated test specimen only. The vertical extensions for the 'SD' configuration shows that the embedment of the arrestment key does reduce the extension a bit for the 1-inch delamination case but is able to flex a bit more for the 2-inch delamination case. The horizontal extension for the 'SD' configurations are a bit lower than the 'ND' configuration, which means the structures fail a bit earlier and the buckled face sheet does not displace as much as it could.

The 'KD' configurations had an issue with the manufacturing. The 'KD' configuration has a higher loading capacity; most likely attributed to higher amount of epoxy content in the structure. The introduction of the delamination into the layup was creating gaps where epoxy would fill up during resin infusion. Overall, the 'KD' configurations are able to maintain the 
majority of the loading and show a bit more vertical extension than the 'SD' configuration. The horizontal extensions differ as well when it experiences more displacement before failure.

\subsubsection{Comparison between 'ID', 'PL', and 'HD' Configurations}

Table 36: Comparison between 'ID', 'PL', and 'HD' Configurations

\begin{tabular}{|c|c|c|c|c|}
\hline Case & $\begin{array}{c}\text { Slenderness } \\
\text { Ratio }\end{array}$ & $\begin{array}{c}\text { Maximum Vertical } \\
\text { Extension }\end{array}$ & $\begin{array}{c}\text { Maximum } \\
\text { Load }\end{array}$ & $\begin{array}{c}\text { Maximum } \\
\text { Horizontal Extension }\end{array}$ \\
\hline- & - & in & lbf & in \\
\hline PL1.0 & 65.63386 & 0.03422 & 237.40629 & 0.03618 \\
\hline PL2.0 & 65.39931 & 0.08476 & 106.79098 & 0.14095 \\
\hline HD1.0 & 64.53014 & 0.03443 & 280.36569 & 0.01353 \\
\hline HD2.0 & 65.27143 & 0.20916 & 101.60259 & 0.43025 \\
\hline 1.0 ID & 65.69982 & 0.03675 & 267.32638 & 0.00986 \\
\hline 2.0 ID & 65.89214 & 0.04118 & 132.60860 & 0.04519 \\
\hline
\end{tabular}

These configurations in this section would not be viable for the practical use of maintaining structural integrity when there is a delamination in the structure. The 'PL' configuration created stress point concentration at the ends of the arrestment keys near the delaminated region. The 'HD' configuration was useless because the gap between the key and delaminated region caused the inefficiency.

From Table 36, the 'PL' configuration failed at lower loading capacities than the 'ID' control configuration themselves. The extensions differ depending on the delamination size. The 'PL1.0' configuration did have a shorter vertical extension and a larger horizontal extension. Similarly, 'PL2.0' configuration had a larger horizontal extension causing the buckled face sheet to fail at the point concentration.

The 'HD' configuration did not live up to its full potential because of the gap sizing. The 'HD1.0' configuration did show a slight improvement because the delamination size was smaller and the key placement was closer to the center of the test specimen. This differs for the 'HD2.0' configuration where it fails a bit earlier than the '2.0 ID' configuration.

Overall, none of these configurations will be not sufficient in maintaining the structural integrity and are not viable choices in comparison to the other configurations. 


\section{CONCLUSION}

\subsection{Summary of Thesis}

This investigation presents the effects of delamination arrestment keys to the failure modes at the delaminated region exhibited by the composite sandwich structure. There were three control groups and four experimental group configurations that were tested under buckling loading. Each test specimen in the experimental groups was investigated for a 1-inch and 2-inch delamination length. Monotonic testing was also conducted on the control group with and without the arrestment keys to see the advantages and disadvantages between the different experimental configurations.

The key conclusion found from the experimental analysis of the composite sandwich structure is summarized below:

- Having a delamination arrestment key that spans the full anvil length and placed parallel to the load vector provides the best results where the majority of the overall failure load is maintained. For 1-inch delaminated 'SD' configuration test specimens, there is only an $11.8 \%$ drop in load capacity from a non-delaminated 'ND' composite sandwich structure and a $126 \%$ increase in load capacity from the 1 -inch 'ID' configuration.

- The failure modes of the experimental testing differ for the various configurations and delamination sizes. These failure modes range from localized buckling of the face sheet for the control 'ID' configuration to arrestment of the 1-inch delamination for the 'KD' configuration to point load concentrated failure for the 'PL' configurations.

\subsection{Possible Future Works}

For what has been done for the experimental and numerical testing and analysis on this project, more work could be completed to better understand the behavior of the delamination arrestment keys within the composite sandwich structure. This thesis project has been a proof of 
the concept of the delamination arrestment key and has only made a small dent in the research regarding the benefits and use of these arrestment keys. Several suggestions to continue the research are summarized below:

- Different loading conditions such as impact and bending must be done on the composite sandwich structures with the embedded key to further understand the arrestment device. Fatigue loading can also be tested.

- Different cross-sectional shapes of the delamination arrestment keys can be studied. Various cross-sectional shapes such as a triangle or rectangle will differ in how the composite sandwich structures will respond under the specific loading conditions and even reduce stress concentration on the keys. This may also include varying the size of the cross-sectional area.

- Repairs for a delamination arrestment keys must be investigated for situations where damage may impact the key and cause loss of load transfer.

- Change of materials for the composite sandwich structure to see the behavioral trends and see if the assumptions and trends are the same. This will also provide better insight if the arrestment key has to change to support the change in materials.

- More numerical analysis on the composite sandwich structure can be done such as:

$\circ$ Varying the size of the cross-sectional area of the arrestment key in a composite sandwich structure model.

- Subjecting the composite sandwich structure to different loading conditions such as three-point bending and fatigue.

- Looking into the plastic region of the composite sandwich structure. 


\section{REFERENCES}

1. “AD-1.”NASA. Web. 12 Aug. 2010.

$<$ http://mm04.nasaimages.org/MediaManager/srvr?mediafile=/Size4/nasaNAS-2NA/3853/ECN13305.jpg\&userid=1\&username=admin\&resolution=4\&servertyp $\mathrm{e}=J V A \& \mathrm{cid}=2 \& \mathrm{iid}=$ nasaNAS $\& \mathrm{v}$ cid $=$ NA\&usergroup $=$ nasa $\&$ profileid $=9>$.

2. ASTM International. Vol. C. West Conshohocken: ASTM International, 2000.

3. Becker, Wilfried, and Goswami, Sanjib. "The effects of facesheet/core delamination in sandwich structures under transverse loading." Bedfordshire: Cranfield University, 2001.

4. "Boat Haul." Boats Online. Web. 14 Aug. 2010.

<http://209.51.145.59/ad_img/5/1/9/1/8/8_2.jpg>.

5. "Bonded Repair of Aircraft Composite Sandwich Structures." DOT/FAA/AR-03/74.

Office of Aviation Research. February 2004. Web. 19 Aug. 2010

$<$ http://www.tc.faa.gov/its/worldpac/techrpt/ar03-74.pdf>.

6. “Composites Diagram.” Brandon Reynante. Web. 12 Aug. 2010.

$<$ http://www.brandonreynante.com/Composites_diagram.jpg >.

7. “Composite Repair.” Hexcel Composites. 2 July. 2010. Web. 19 Aug. 2010

$<$ http://home.iitk.ac.in/ mohite/Composite_Repair.pdf>.

8. "Fabrics Data Sheets." www.hexcel.com. Hexcel. Web. 12 Aug. 2010.

$<$ http://www.hexcel.com/www.hexcel.com/Templates/DataSheets_DGFabrics.as px?NRMODE=Published\&NRORIGINALURL=/Products/Downloads/Fabrics\% 2bDG $\% 2 \mathrm{bData} 2 \mathrm{bSheets.htm} \% 3 \mathrm{fmap} \% 3 \mathrm{dP} 1 \% 26 \mathrm{t} \% 3 \mathrm{dFiberglass} \% 2 \mathrm{bData} \% 2 \mathrm{bSh}$ eets\&NRNODEGUID $=\{4 \mathrm{FE} 1348 \mathrm{~A}-06 \mathrm{EE}-44 \mathrm{E} 1-\mathrm{A} 944-$

9B9576F1E94A $\} \&$ NRCACHEHINT=NoModifyGuest $>$.

9. "Fiber Volume Fraction of a Composite Materials." About Composites - Learn about Plastic and Composite Materials. Web. 24 Aug. 2010. $<$ http://composite.about.com/library/glossary/f/bldef-f2200.htm>.

10. "Golden Gate Bridge: San Francisco Views." San Francisco Underground Travel. Web. 16 Aug. 2010. <http://www.sftravel.com/goldengatebridge.html>.

11. "G1 Solar Panel.” ICO Global Communications. Web. 13 Aug. 2010.

$<$ http://www.ico.com/_about/tech/_img/g1_solar_panel_1.jpg >. 
12. Huang, Shu-Mei., and Hwang, Shun-Fa. "Postbuckling behavior of composite laminates with two delaminations under uniaxial compression." Taiwan: National Yunlin University of Science and Technology, 2004.

13. "Hybrid Skin.” RSA F3B. Web. 14 Aug. 2010. <http://3.bp.blogspot.com/_hH9yoQCmv5g/Rs_sc3gmO5I/AAAAAAAAAPI/y4 av7aqr0ag/s320/Hybrid+skin.JPG $>$.

14. "Introduction To the Glastar Kit." Glasair Aviation. Web. 12 Aug. 2010. $<$ http://www.glasairaviation.com/kitcontentglastar.html>.

15. "Locus HTT Plethore.” Super Car Specs. Web. 12 Aug. 2010. $<$ http://www.supercarspecs.com/images/locus/htt_plethore/mainblog.jpg>.

16. "Maritimo Aegean 60." Yacht and Boat. Web. 12 Aug. 2010. $<$ http://www.yachtandboat.com.au/images/Boat\%20Shows/Maritimo\%20Aegean $\% 2060 \% 20$ Boat\%20Show.jpg>.

17. "Mesh 1.” YZGTBX. Web. 12 Aug. 2010. <http://www.yzgtbx.com/cp/html/images/1188705100.jpg>.

18. “Mesh 2.” YZGTBX. Web. 12 Aug. 2010. <http://www.yzgtbx.com/cp/html/images/1188703338.jpg>.

19. “Mesh 3.” YZGTBX. Web. 12 Aug. 2010. <http://www.yzgtbx.com/cp/html/images/1188704894.jpg>.

20. "Rebar Oven Floor.” DIY Networks. Web. 12 Aug. 2010. <http://img.diynetwork.com/DIY/2008/04/17/dind103_1fb_Rebar_OvenFloor_le ad.jpg $>$.

21. "Sandwich Composite." Railway Technology. Web. 13 Aug. 2010. <http://www.railwaytechnology.com/contractor_images/diab/1-sandwich-composite.jpg >.

22. "Sandwich Composite 2." TREMA. Web. 13 Aug. 2010. $<$ http://aquacasa.eu/Afbeelding1.jpg $>$.

23. "Satellite Akebono.” ASC-Canadian Space Agency. Web. 12 Aug. 2010. $<$ http://www.asc-csa.gc.ca/images/satellite-akebono.jpg>.

24. "SPAC Minotaur Rocket Launch.” Defense Industry Daily. Web. 12 Aug. 2010. $<$ http://media.defenseindustrydaily.com/images/SPAC_Minotaur_Rocket_Launc 
h_lg.jpg >

25. Tran, Tony,. et al.. "Monotonic Compression Buckling Response of Initially Delaminated Composite under Different Temperatures.” San Luis Obispo: Cal Poly. 2009.

26. "Virgin American Airline's Interior." Wikipedia. Web. 13 Aug. 2010.

$<$ http://upload.wikimedia.org/wikipedia/commons/1/1e/Virgin_America_airplane _interior.jpg>.

27. "VWR.COM - Product Page." Welcome to VWR International. VWR. Web. 25 Aug. 2010.<https://www.vwrsp.com/catalog/product/index.cgi?object_id=0008675\&cl ass_id $=5035352>$.

28. "WEST SYSTEM I Epoxy Resins and Hardeners - Physical Properties." Epoxy by the Leading Epoxy Manufacturer I WEST SYSTEM Epoxy. Web. 12 Aug. 2010. $<$ http://www.westsystem.com/ss/typical-physical-properties/>. 


\section{APPENDIX}

\section{A. Comparison of All Configurations}

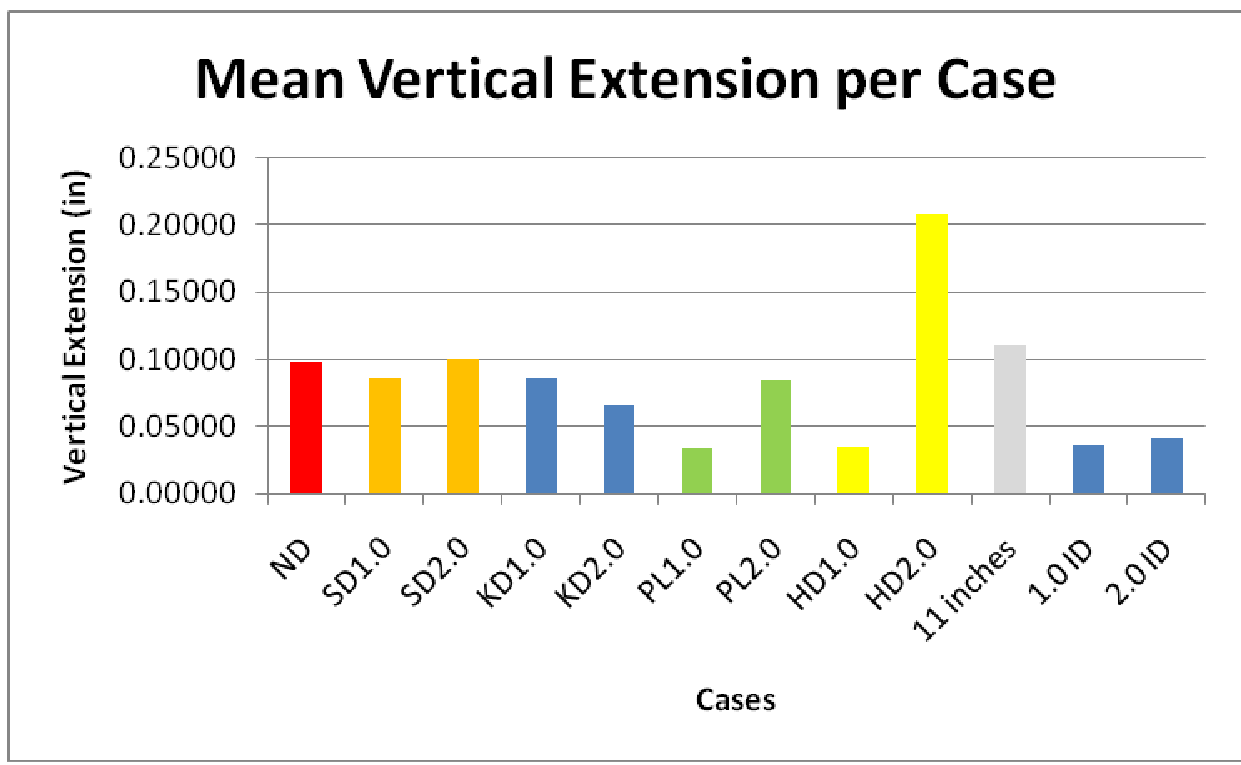

Figure 180: Mean Vertical Extension per Case

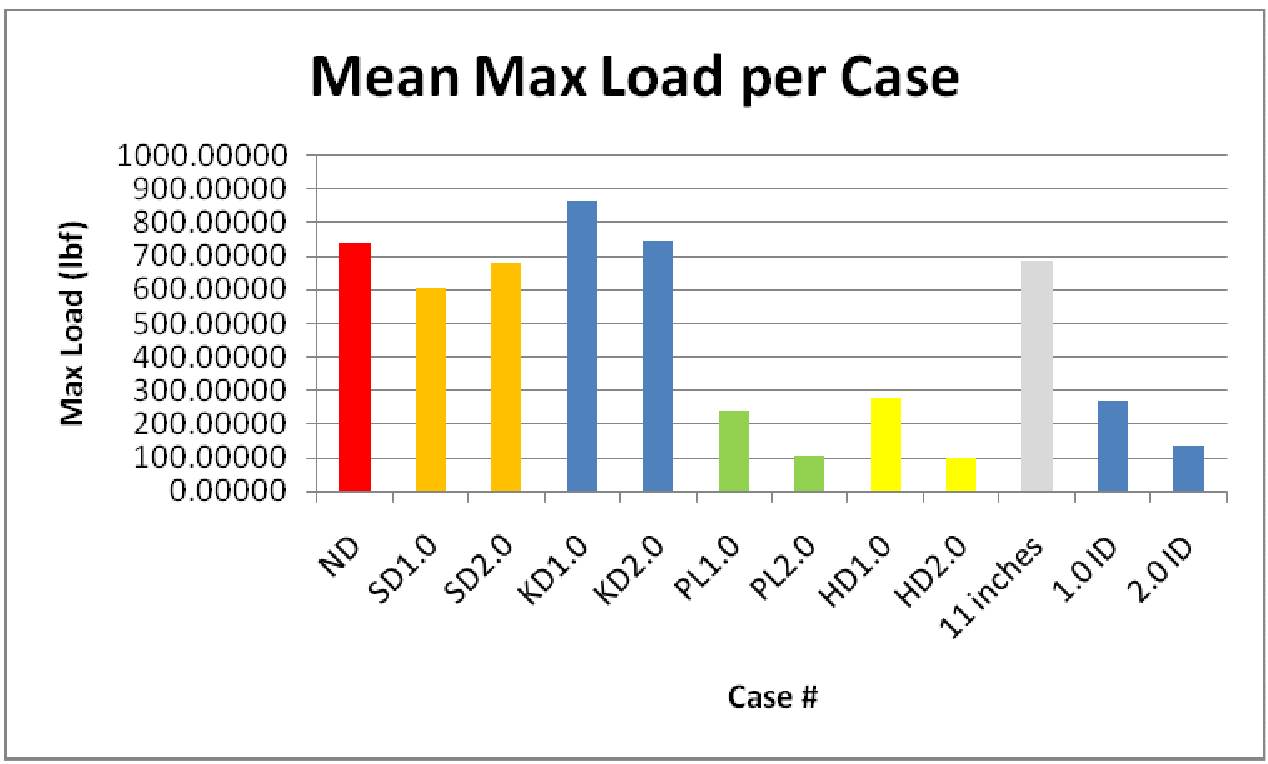

Figure 181: Mean Ultimate Load per Case 


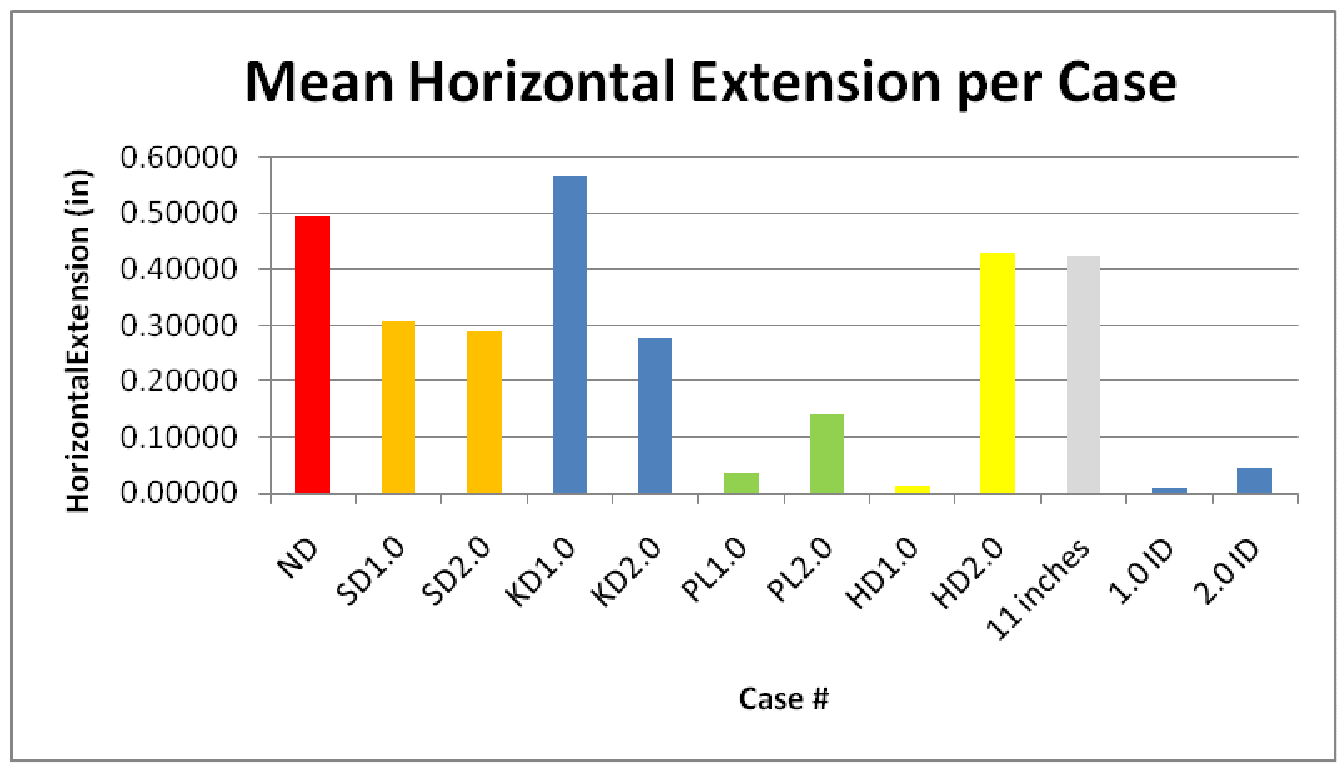

Figure 182: Mean Max Horizontal Extension per Case

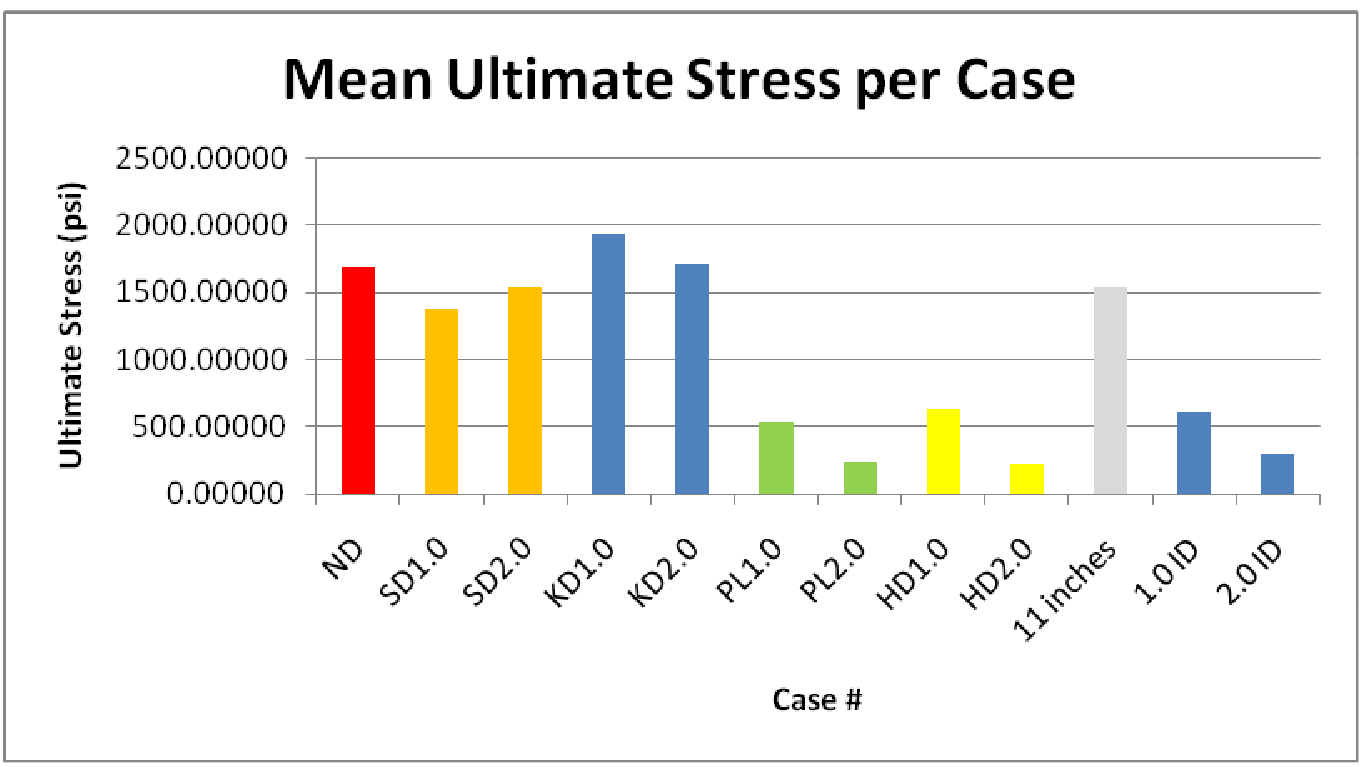

Figure 183: Mean Ultimate Stress per Case

B. Thermolyne Type 1300 Muffle Furnace Specifications [31]

\begin{tabular}{|l|l|}
\hline Maximum Temperature & 2012 degrees Fahrenheit \\
\hline Type 1300 Chamber Dimensions & 4 " by 3.75 " by $4.25 "$ \\
\hline Overall Dimensions & 8 " by 12.5 " by 8.5 " \\
\hline Weight & $13.5 \mathrm{lbs}$ \\
\hline Electrical & 120 or $240 \mathrm{~V}, 50 / 60 \mathrm{~Hz}$ \\
\hline
\end{tabular}

